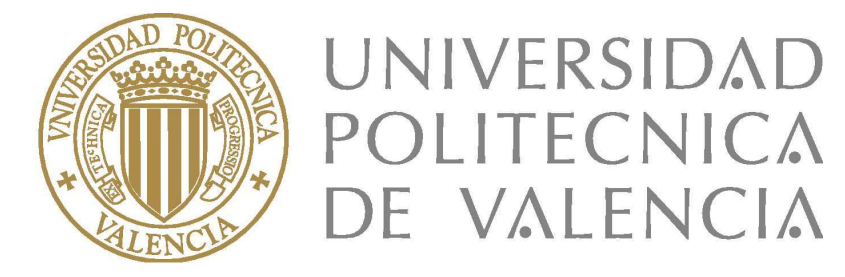

\author{
UNIVERSIDAD POLITÉCNICA DE VALENCIA
}

Departamento de Comunicaciones

\title{
FULL-WAVE ANALYSIS AND DESIGN OF PASSIVE \\ MICROWAVE AND MILLIMETRE-WAVE DEVICES \\ BASED ON DIELECTRIC-LOADED CAVITY RESONATORS
}

\author{
Jordi Gil Raga
}

Directores:

Dr. Vicente E. Boria Esbert (Universidad Politécnica de Valencia)

Dr. Benito Gimeno Martínez (Universitat de València Estudi General)

Tesis Doctoral presentada en la Universidad Politécnica de Valencia para la obtención del título de Doctor Ingeniero de Telecomunicación

Valencia, Diciembre 2009 

A mis padres, a mis abuelas, mis hermanas, a Neus. 



\section{Agradecimientos}

El 2001 fue un año especial, un año de incertidumbres y mucha actividad. Recién terminada la carrera de físicas, las perspectivas de futuro abrían ante mí nuevos interrogantes. Había llegado el excitante y temido momento de tomar decisiones que presumiblemente cobrarían una relevancia crucial en mi vida. Traté de aprovechar todos los proyectos que tenía en mente sin mucho orden ni concierto. No tenía muy claro cuál acabaría siendo mi futuro profesional, pero estaba convencido de que alguno de los caminos que había escogido me llevaría a buen puerto. Y sin embargo, la casualidad me tenía reservada una sorpresa. La casualidad quiso que una mañana de enero, en la que me acerqué a la secretaría de la facultad, me fijase en un cartel que anunciaba una oferta de trabajo en un proyecto de investigación europeo. Para más información preguntad por Benito Gimeno, se leía. La casualidad también quiso que, a pesar de que el tema de investigación no encajaba en mis expectativas del momento, me animase a pasarme a hacerle una visita a aquel profesor a quien, y esto no por casualidad, valoraba mucho. De aquella visita recuerdo una imagen que se repetiría en numerosas ocasiones en años posteriores, la de un entrañable y divertido profesor sentado en su silla de investigador espacial al fondo de su despacho, tras unas gafas asombradas de todo lo que ocurre al otro lado de las lentes, y envuelto entre grandes papeles con enormes fórmulas matemáticas. Benito me explicó que, tanto él como Vicente, se habían embarcado en una colaboración científica con otras instituciones europeas. Ambos me ofrecieron la posibilidad de hacer una estancia de aproximadamente tres años en una empresa italiana, en Pisa.

Unos meses después de aquel encuentro, emprendí mi viaje. Aquel proyecto me permitió conocer Italia, nuevos amigos, trabajar con distintos grupos de investigación, fue una etapa en la que aprendí muchísimo. Pero lo que más marcó mi futuro, fue la pasión que aquellos dos profesores mostraban por lo que hacían, su forma de trabajar en equipo, la manera en la que se preocupaban por que la gente con la que colaboraban estuviese a gusto. Todo aquello supuso el punto de partida de mi trabajo doctoral, decidí que quería volver a Valencia para poder trabajar con Benito y Vicente. A ellos les quiero expresar mi reconocimiento y mi gratitud por todo lo que han hecho por mí estos años. Espero que podamos seguir trabajando juntos por mucho tiempo. Que se sigan repitiendo mis visitas al despacho de Beni, y que sean muchas las reuniones en las que Vicente siempre encuentre alguna solución, y en las que, por muy duras que sean, siempre tengamos tiempo para hacernos unas risas. Sois dos investigadores extraordinarios, dos grandes amigos.

Durante los dos primeros años tras mi vuelta a Valencia, mi trabajo estuvo siempre a caballo entre la Universitat de València y la Universitat Politècnica de València. No puedo evitar esbozar una sonrisa divertida al recordar los buenos momentos vividos en el despacho compartido con Máriam, Stephan, Fermín, Morro y Luis, o las pausas del café con Martina, Pere, Juan Ángel, Arturo, Pepe, y Antonio.

A Pere y Martina les agradezco especialmente que me animasen a colaborar, junto con ellos, en Joves Invesitgadors. Una asociación que lucha por una carrera investigadora digna y por el reconocimiento social que esta profesión merece. En ella, me econtré con buenos 
amigos, de letras y ciencias, como Dani, Isabel, Gloria , Pepo, Amparo, Olga y Juan Antonio. Con Pere a la cabeza organizamos las terceras Jornadas de Jóvenes Investigadores en Valencia, fue todo un éxito. Gracias a todos ellos, a los que estaban antes y a los que han llegado después por dedicar sus esfuerzos a mejorar las condiciones laborales de los que vienen detrás.

Más tarde constituimos la empresa, Aurorasat, aventura en la que nos embarcamos Vicente, Benito, Carlos, Alejandro y yo. Con Carlos ya había trabajado en el marco del proyecto europeo, cuando él estaba en Alemania y yo en Italia. Habíamos hablado en numerosas ocasiones de la posibilidad de volver a casa y tratar de crear algo a partir de lo que habíamos aprendido fuera, hoy compartimos despacho en Aurorasat. Y quiero agradecerle su labor incansable al frente de ésta, lo cual ha permitido que yo dedicase el tiempo necesario a mi trabajo doctoral. La empresa que con tanto esfuerzo y trabajo sacamos adelante día a día gracias a un excelente grupo de colegas y amigos, Sergio, Jaime, Teresa y los Javis, Monge, Pérez Soler y Sanz. Sin olvidar la colabaroción que mantenemos con la gente de la universidad formando un gran equipo: Santi, Pablo, Máriam, Stephan, Òscar, Mario, Marta, Ángel y Quesada.

No quiero dejar de mencionar la ayuda que durante este tiempo me han prestado Fermín y Ángel San Blas, perros viejos ya en esto del método BIR-ME, siempre me han echado un cable cuando lo he solicitado. Así como el inestimable apoyo del profesor Marco Bressan, de la Università di Pavia, sin quien las funciones de Green pueden llegar a ser un mundo inescrutable. También quisiera agradecer la ayuda que el profesor de la facultad de matemáticas Manolo Maestre nos ha brindado cuando alguna integral se ha complicado más de la cuenta. Y la paciencia que Michael Mattes, de la École Polytechnique Fédérale de Lausanne, tuvo cuando me enseñó los entresijos del FEST.

A los amigos de toda la vida, a los del Roser, entre los que incluyo a Romualdo y familia, a Kike, Rafa, Alejandro, a los amigos de la carrera, Pedro, Alonso, Yubero, José Antonio, Manolo, José Javier, Ana Teresa, Nácher, gracias a todos por los buenos momentos, por estar ahí, gracias.

Por último, quiero agradecer todo esto a quienes lo han hecho posible, mi familia. A mis padres, Ángel y Marisa, sin cuyo cariño y dedicación nada de esto hubiera sucedido. Vosotros me inculcasteis la importancia del estudio desde mi infancia, siempre habéis sido el ejemplo a seguir. A mis abuelas, Luisa y Angelita, que siempre han seguido mis pasos, y han velado por mí. A mis hermanas, Mireia e Irene, por quererme, por ser la otra parte del puzzle.

Y a Neus, mi compañera, a quien amo, a quien admiro por su capacidad de esfuerzo y su bondad. Gracias por tu amor, por tu apoyo incondicional, por tu paciencia, por ser como eres. Neus, preciosa, como dice el cantante, te cambio mi corazón por el tuyo, para mirarlo y mirarlo.

Gracias a todos por esta historia. 


\section{Resumen}

Desde los primeros estudios desarrollados por S. B. Cohn a finales de los años 60 , los resonadores dieléctricos, con factor de calidad elevado, han sido utilizados para el diseño de filtros paso-banda de microondas. A partir de la aparición en los años 70 de materiales dieléctricos con las propiedades eléctricas y estabilidad térmica idóneas, los resonadores dieléctricos se convirtieron en elementos clave en numerosos diseños de filtros. De hecho, esta tecnología se encuentra frecuentemente en sistemas de comunicaciones móviles y por satélite debido a sus ventajas en términos de reducción de masa y volumen, bajas pérdidas, y estabilidad térmica. Por todo ello, el análisis y diseño riguroso de este tipo de filtros ha suscitado un gran interés en la literatura técnica.

El objetivo principal de esta tesis doctoral es el desarrollo de una técnica modal eficiente para caracterizar el comportamiento electromagnético de cavidades resonantes cargadas con dieléctricos. Para ello, se presenta una nueva formulación de ecuación integral en el espacio de estados basada en el método BI-RME (del inglés, boundary integral-resonant mode expansion). En dicha formulación, para resolver la ecuación integral de volumen planteada, el resonador dieléctrico se caracteriza de manera rigurosa por medio de las densidades de carga y corriente de polarización equivalentes definidas en el volumen del objeto dieléctrico. Siguiendo este método, los modos resonantes de las cavidades se obtienen a través de la solución de un problema lineal de autovalores. Así mismo, se obtiene la matriz generalizada de admitancias de la cavidad resonante cargada con dieléctrico como una expansión en serie de polos en el dominio de la variable de Laplace. De esta manera, la respuesta electromagnética de las cavidades resonantes puede resolverse en un rango amplio de puntos de frecuencia haciendo uso de un reducido esfuerzo computacional, y evitando así, cálculos intensos en cada punto de frecuencia.

La formulación desarrollada ha sido aplicada al análisis de cavidades rectangulares cargadas con resonadores dieléctricos cilíndricos. Se ha integrado el código implementado en una herramienta CAE (del inglés, Computer Aided Engineering ) para el diseño y análisis de componentes pasivos de microondas y ondas milimétricas. Esta herramienta CAE es de propósito general y está basada en técnicas modales avanzadas. De esta manera, se ha desarrollado una eficeinte herramienta informática que permite el análisis riguroso de filtros con resonadores dieléctricos. De hecho, distintos filtros paso-banda y elimina banda han sido diseñados para validar esta nueva herramienta. Los resultados numéricos obtenidos han sido comparados existosamente con literatura técnica, así como con los resultados proporcionados por una reconocida herramienta comercial basada en el método de elementos finitos. Por tanto, se ha probado que el método implementado es preciso y computacionalmente eficiente, porporcionando por ello, una herramienta idónea para el diseño optimizado de filtros en guía onda con resonadores dieléctricos. 



\section{Resum}

Des dels primers estudis desenvolupats per S. B. Cohn a finals dels anys 60, els ressonadors dielèctrics, amb factor de qualitat elevat, han sigut emprats per al disseny de filtres passa-banda de microones. Arran de l'aparició en els anys 70 de materials dielèctrics amb les propietats elèctriques i l'estabilitat tèrmica idònies, els ressonadors dielèctrics s'han convertit en elements clau en nombrosos dissenys de filtres. De fet, aquesta tecnologia es troba freqüentment en sistemes de comunicacions mòbils i per satèl-lit degut a les seues avantatges en termes de reducció de massa i volum, baixes pèrdues, i estabilitat tèrmica. Per tot això, l'anàlisi i disseny rigorós d'aquest tipus de filtres ha suscitat un gran interès en la literatura tècnica.

L'objetiu principal d'aquesta tesi doctoral és el desenvolupament d'una tècnica modal eficient per a caracteritzar el comportament electromagnètic de cavitats ressonants carregades amb dielèctrics. Per això, es presenta una nova formulació d'equació integral en l'espai d'estats basada en el mètode BI-RME (de l'anglés, boundary integral-resonant mode expansion). En aquesta formulació, per a resoldre l'equació integral de volum plantejada, el ressonador dielèctric es caracteritza de manera rigorosa per mitjà de les densitats de càrrega i corrent de polarització equivalentes definides en el volum de l'objetce dielèctric. Seguint aquest mètode, els modos ressonants de les cavitats s'obtenen a través de la solució d'un problema lineal de autovalors. Així mateix, s'obté la matriu generalitzada d'admitàncies de la cavitat ressonant carregada amb dielèctric com una expansió en sèrie de pols en el domini de la variable de Laplace. D'aquesta manera, la resposta electromagnètica de les cavitats ressonants pot resoldre's en un rang ample de punts de freqüència fent ús d'un reduït esforç computacional, evitant així, càlculs intensos en cada punt de freqüència.

La formulació desenvolupada ha sigut aplicada al anàlisi de cavitats rectangulars carregades amb ressonadors dielèctrics cilíndrics. S'ha integrat el codi implementat en una eina CAE (de l'anglés, Computer Aided Engineering ) per al disseny i anàlisi de components passius de microones i ones milimètriques. Aquesta eina $\mathrm{CAE}$ és de propòsit general i està basada en tècniques modals avançades. D'aquesta manera, s'ha desenvolupat una eficient eina informàtica que permet l'anàlisi rigorós de filtres amb ressonadors dielèctrics. De fet, distints filtres passa-banda y elimina-banda han sigut dissenyats per validar aquesta nova eina. Els resultats numèrics obtinguts han sigut comparats reeixidament amd la literatura tècnica, així com amb els resultats proporcionats per una reconeguda eina comercial basada en el mètode d'elements finits. Per tant, s'ha provat que el mètode implementat és precís i computacionalment eficient, porporcionant degut a això, una eina idònia per al disseny optimitzat de filtres en guia ona amb ressonadors dielèctrics. 



\section{Abstract}

Since the first studies developed by S. B. Cohn in the late 1960s, high-Q dielectric resonators have been employed to design microwave bandpass filters. Once new dielectric materials with suitable electrical properties and temperature stability were proposed in the 1970s, dielectric resonators became a key element in many filtering applications. Indeed, dielectric-loaded waveguide filters are frequently found in satellite and mobile communication systems, due to their advantages in terms of mass and volume reduction, low losses, and thermal stability. For these reasons, the full-wave analysis and design of such filters has deserved considerable attention in the technical literature.

The main objective of this $\mathrm{PhD}$ thesis is the development of an efficient modal technique to characterise the electromagnetic behaviour of dielectric-loaded cavity resonators. For such purpose, a new state-space integral-equation (SS-IE) formulation in the $s$-domain, based on the boundary integral-resonant mode expansion (BI-RME) method, is presented. In order to solve the volume integral equation proposed, the dielectric resonator is rigorously characterized by means of the electric equivalent polarization charge and current densities defined in the volume of the dielectric object. Following this method, the resonant modes of the considered cavities are obtained through the solution of a linear matrix eigenvalue problem. Furthermore, a pole expansion of the generalized admittance matrix of the dielectric-loaded cavity is obtained in the domain of the Laplace variable. Hence, the electromagnetic behaviour of the cavity resonators can be solved in a wide and dense frequency range with a very reduced computational effort, avoiding to perform intensive computations at each frequency point.

The formulation developed has been applied to analyse rectangular cavities loaded with cylindrical dielectric resonators. The implemented code has been integrated into a Computer Aided Engineering (CAE) tool for the analysis and design of passive microwave and millimetre waves components. This CAE tool is a general purpose electromagnetic solver based on adevanced modal techniques. Thus, a very efficient software tool for the full-wave analysis of dielectric resonator filters has been developed. Indeed, different bandpass and stopband single-mode filters have been designed using the new software tool. The numerical results provided by this tool have been successfully compared with those included in the technical literature, as well as with those provided by a well-known commercial code based on the finite-element method. It has been proved that the implemented method is very accurate and computationally efficient, thus making it very suitable for the optimized design of waveguide filters including dielectric resonators. 



\section{Index}

1 Introduction 1

1.1 Motivation and state of the art ............... 1

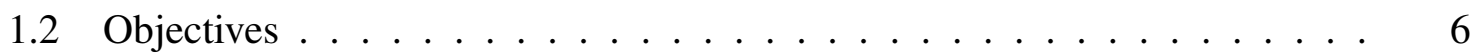

1.3 Thesis structure ...................... 7

2 Theory of Cavity Resonators Review 9

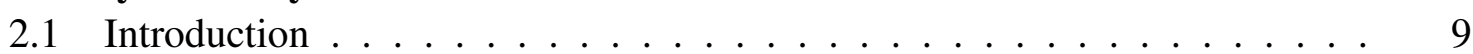

2.2 The expansion of electromagnetic fields in cavity resonators . . . . . . 10

2.2.1 Electric eigenvectors . . . . . . . . . . . . . . . . 12

2.2.2 Magnetic eigenvectors . . . . . . . . . . . . . . . 14

2.3 Forced oscillations in cavity resonators . . . . . . . . . . . . . . 15

2.4 Electric Green's functions in the source region. Singularity extraction . . . 18

2.4.1 Decomposition of the electric dyadic Green's function and singularity extraction ...................... 19

2.4.2 Physical meaning of the electric dyadic Green's function decomposition: The Coulomb Gauge. . . . . . . . . . . . . . . . . . 22

2.5 Scalar and vector potentials (Coulomb gauge) . . . . . . . . . . . . . 24

2.6 Potential Green's functions . . . . . . . . . . . . . . . . . . . 27

2.7 Hybrid representation of the electromagnetic field in an ideal cavity . . . . 30

3 State-Space Integral-Equation Formulation based on the BI-RME Method 33

3.1 Introduction . . . . . . . . . . . . . . . . . 33

3.2 Dielectric obstacle characterization by means of the equivalent polarization

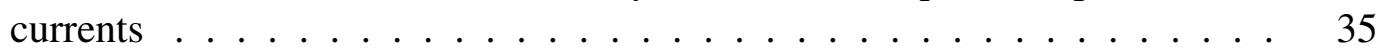

3.3 State-Space Integral-Equation Approach . . . . . . . . . . . . . . . . . . . 39

3.4 Resonant modes of the dielectric-loaded cavity . . . . . . . . . . . . . 46

3.5 Generalized admittance matrix in the form of pole expansions . . . . . . 48

4 Application of the State-Space Integral-Equation Formulation to the analysis of a rectangular cavity loaded with a dielectric cylinder $\quad \mathbf{5 1}$

4.1 Introduction . . . . . . . . . . . . . . . . . 51

4.2 Basis functions . . . . . . . . . . . . . . . 53

4.3 Calculation of the matrices used in the MoM . . . . . . . . . . 60 
4.3.1 Computation of the $\mathbf{D}$ matrix . . . . . . . . . . . . . . 60

4.3.2 Computation of the $\mathbf{S}$ matrix . . . . . . . . . . . . . . 64

4.3.3 Computation of the $\mathbf{C}$ matrix . . . . . . . . . . . 71

4.3.4 Computation of the $\mathbf{L}$ matrix . . . . . . . . . . . . 79

4.3.5 Computation of the $\mathbf{G}, \mathbf{T}, \mathbf{W}$ and $\mathbf{F}$ matrices . . . . . . . . . . 92

5 Analysis of rectangular cavities loaded with a dielectric puck: Resonant fre$\begin{array}{ll}\text { quencies and modal chart } & 105\end{array}$

5.1 Introduction . . . . . . . . . . . . . . . . . . . . 105

5.2 Modal chart . . . . . . . . . . . . . . . . . . . . 108

6 Design of passive microwave filters based on dielectric resonators 129

6.1 Introduction . . . . . . . . . . . . . . . . . . . . . . 129

6.2 Integration into FEST3D $\ldots \ldots \ldots \ldots \ldots \ldots$

6.2 .1 Introduction to FEST3D. . . . . . . . . . . . . . . . 133

6.2.2 Integration of the DR-loaded cavity module into FEST3D. . . . . . 137

6.3 Bandpass and bandstop dielectric resonator filters . . . . . . . . . . . 139

7 Conclusions and future work $\quad 153$

$\begin{array}{ll}\text { APPENDIXES } & 156\end{array}$

A Modal analysis of rectangular cavities and waveguides 157

A.1 Magnetic modal vectors of a rectangular waveguide . . . . . . . . . . . . 157

A.2 Electric and magnetic solenoidal modal vectors of a rectangular cavity . . . 159

A.3 Magnetic Green's functions of a rectangular cavity . . . . . . . . . . 161

B Integrals involving trigonometric functions $\quad 165$

$\begin{array}{ll}\text { C Dyadic Analysis } & 167\end{array}$

D Integrals involved in the computation of Matrix C 171

D.1 Integral involved in the computation of the matrix $\tilde{C}^{(1,1)} \ldots \ldots \ldots \ldots 171$

D.2 Integral involved in the computation of the matrix $\tilde{C}^{(3,3)} \ldots \ldots \ldots \ldots$

D.3 Integral involved in the computation of the matrix $\tilde{C}^{(1,3)} \ldots \ldots \ldots \ldots$

E Properties of the Green's functions used to calculate the L matrix. 179

E.1 Singular dyadic term of the static part of the Green's function for the potential vector . . . . . . . . . . . . . . . . . . . . 182

E.2 Regular dyadic term of the static part of the Green's function for the potential vector .......................... 188

F Auxiliary series and functions used in the aperture of the access ports 191

F.1 Auxiliary series used in the aperture of the access ports . . . . . . . . . . . 191

F.2 Auxiliary functions used in the aperture of the access ports . . . . . . . . 192 
G Publications related with the PhD thesis 195

BIBLIOGRAPHY 


\section{Figure Index}

2.1 Different types of cavity resonators. . . . . . . . . . .

3.1 The basic building block under study: a dielectric resonator arbitrarily placed inside a cavity resonator connected to access ports. . . . . . . . . .

4.1 The basic building block under study: a cylindrical dielectric resonator arbitrarily placed inside a rectangular cavity connected to two rectangular waveguides. . . . . . . . . . . . . . . . . . .

4.2 Simple models to analyse DR . . . . . . . . . . . . . . .

4.3 Diagram of the basis functions used in the polarization vector expansion: The solenoidal eigenvectors of the cylindrical cavity (with diameter $d$ and height $h$ ) containing the DR are used both with perfect electric walls (PEW) and perfect magnetic walls (PMW).

4.4 Basis functions used by the MoM in equatorial plane of the DR. . . . . . . 55

4.5 Basis functions used by the MoM in meridian plane of the DR. Figs. 4.5(a)4.5(d) show the plane with $\phi=0$, whereas Figs. 4.5(e)-4.5(h) show the

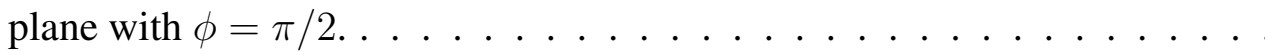

4.6 Cartesian and polar coordinate systems associated to the rectangular cavity and to the cylindrical DR, respectively. . . . . . . . . . . . .

4.7 Rectangular cavity loaded with a cylindrical DR opened by its five access ports. . . . . . . . . . . . . . . .

4.8 Rectangular cavity loaded with a cylindrical DR opened by its five access ports.

5.1 Rectangular cavity loaded with a cylindrical dielectric resonator.

5.2 Electric field distribution for the first hybrid mode, $H E_{12}$, at the equatorial plane. Rectangular cavity dimensions: $a=25.4 \mathrm{~mm}, b=25.4 \mathrm{~mm}, c=$ $23.37 \mathrm{~mm}$; DR: $d=16.61 \mathrm{~mm}, h_{1}=5.54 \mathrm{~mm}, \varepsilon_{r_{1}}=38$; DR support: $h_{2}=6.99 m m, \varepsilon_{r_{2}}=1 \ldots \ldots \ldots \ldots \ldots$

5.3 Resonant frequencies of the two hybrid modes $H E_{12}$ as a function of the length, dimension $c$, of the rectangular cavity. Rectangular cavity dimensions: $a=25.4 \mathrm{~mm}, b=25.4 \mathrm{~mm}$; DR: $d=16.61 \mathrm{~mm}, h_{1}=5.54 \mathrm{~mm}$, $\varepsilon_{r_{1}}=38$; DR support: $h_{2}=6.99 \mathrm{~mm}, \varepsilon_{r_{2}}=1$. 
5.4 Electromagnetic field distribution for the first hybrid mode, $H E_{12}$, in the meridian plane with $\varphi=\pi / 2$. Rectangular cavity dimensions: $a=25.4 \mathrm{~mm}$, $b=25.4 \mathrm{~mm}, c=23.37 \mathrm{~mm}$; DR: $d=16.61 \mathrm{~mm}, h_{1}=5.54 \mathrm{~mm}$, $\varepsilon_{r_{1}}=38$; DR support: $h_{2}=6.99 \mathrm{~mm}, \varepsilon_{r_{2}}=1 . \ldots \ldots . . . .$.

5.5 Electromagnetic field distribution for the $T E_{01}$ mode. Rectangular cavity dimensions: $a=25.4 \mathrm{~mm}, b=25.4 \mathrm{~mm}, c=23.37 \mathrm{~mm}$; DR: $d=$ $16.61 \mathrm{~mm}, h_{1}=5.54 \mathrm{~mm}, \varepsilon_{r_{1}}=38$; DR support: $h_{2}=6.99 \mathrm{~mm}, \varepsilon_{r_{2}}=1$. .

5.6 Electromagnetic field distribution in the equatorial plane for the $H E_{11}^{(e)}$ mode. Rectangular cavity dimensions: $a=36 \mathrm{~mm}, b=30 \mathrm{~mm}, c=40 \mathrm{~mm}$; DR: $d=30 \mathrm{~mm}, h_{1}=10 \mathrm{~mm}, \varepsilon_{r_{1}}=45$; DR support: $h_{2}=7.5 \mathrm{~mm}, \varepsilon_{r_{2}}=1$. .

5.7 Electromagnetic field distribution in the meridian plane with $\varphi=\pi / 2$ for the $H E_{11}^{(e)}$ mode. Rectangular cavity dimensions: $a=36 \mathrm{~mm}, b=30 \mathrm{~mm}$, $c=40 \mathrm{~mm}$; DR: $d=30 \mathrm{~mm}, h_{1}=10 \mathrm{~mm}, \varepsilon_{r_{1}}=45$; DR support: $h_{2}=$ $7.5 \mathrm{~mm}, \varepsilon_{r_{2}}=1$.

5.8 Electromagnetic field distribution for the $T M_{01}$ mode. Rectangular cavity dimensions: $a=36 \mathrm{~mm}, b=30 \mathrm{~mm}, c=40 \mathrm{~mm}$; DR: $d=30 \mathrm{~mm}$, $h_{1}=10 \mathrm{~mm}, \varepsilon_{r_{1}}=45$; DR support: $h_{2}=7.5 \mathrm{~mm}, \varepsilon_{r_{2}}=1 . \ldots \ldots$

5.9 Study of the first six resonant modes of a rectangular cavity loaded with a cylindrical dielectric resonator as a function of the $d / h_{1}$ ratio. Rectangular cavity dimensions: $a=36 \mathrm{~mm}, b=30 \mathrm{~mm}, c=40 \mathrm{~mm}$; DR: $\varepsilon_{r_{1}}=45$; DR support: $h_{2}=7.5 \mathrm{~mm}, \varepsilon_{r_{2}}=1$.

5.10 Study of the first six resonant modes of a rectangular cavity loaded with a cylindrical dielectric resonator as a function of the DR relative permittivity. Rectangular cavity dimensions: $a=25.4 \mathrm{~mm}, b=25.4 \mathrm{~mm}, \mathrm{c}=$ $23.37 \mathrm{~mm}$; DR: $d=16.8 \mathrm{~mm}, h_{1}=8.4 \mathrm{~mm}$; DR support: $h_{2}=6.985 \mathrm{~mm}$, $\varepsilon_{r_{2}}=1 \ldots \ldots \ldots \ldots \ldots \ldots \ldots$

5.11 Electric field distribution in the equatorial plane of a rectangular cavity loaded with an off-centred dielectric resonator. Rectangular cavity dimensions: $a=$ $30 \mathrm{~mm}, b=25.4 \mathrm{~mm}, c=40 \mathrm{~mm}$; DR: $h_{1}=6.43 \mathrm{~mm}, d_{1}=18 \mathrm{~mm}$, $\varepsilon_{r_{1}}=50$; DR support: $h_{2}=11 \mathrm{~mm}, \varepsilon_{r_{2}}=1$, positioned at $a_{o}=17.5 \mathrm{~mm}$, $c_{o}=27.5 \mathrm{~mm}$.

5.12 Electromagnetic field distribution in the equatorial plane of a rectangular cavity loaded with an off-centred dielectric resonator. Rectangular cavity dimensions: $a=30 \mathrm{~mm}, b=25.4 \mathrm{~mm}, c=40 \mathrm{~mm}$; DR: $h_{1}=6.43 \mathrm{~mm}$, $d_{1}=18 \mathrm{~mm}, \varepsilon_{r_{1}}=50$; DR support: $h_{2}=11 \mathrm{~mm}, \varepsilon_{r_{2}}=1$, positioned at $a_{o}=17.5 \mathrm{~mm}, c_{o}=27.5 \mathrm{~mm} \ldots \ldots \ldots \ldots$

5.13 Magnetic field distribution in the equatorial plane of a rectangular cavity loaded with an off-centred dielectric resonator. Rectangular cavity dimensions: $a=30 \mathrm{~mm}, b=25.4 \mathrm{~mm}, c=40 \mathrm{~mm}$; DR: $h_{1}=6.43 \mathrm{~mm}$, $d_{1}=18 \mathrm{~mm}, \varepsilon_{r_{1}}=50$; DR support: $h_{2}=11 \mathrm{~mm}, \varepsilon_{r_{2}}=1$, positioned at $a_{o}=17.5 \mathrm{~mm}, c_{o}=27.5 \mathrm{~mm} \ldots \ldots \ldots \ldots$ 
5.14 Convergence study: Resonant frequencies as a function of the number of basis functions (Q) used by the MoM. Rectangular cavity dimensions: $a=$ $25.4 \mathrm{~mm}, b=25.4 \mathrm{~mm}, c=23.368 \mathrm{~mm}$; DR: $h_{1}=6.43 \mathrm{~mm}, d=19.23 \mathrm{~mm}$, $\varepsilon_{r_{1}}=38 ;$ DR support: $h_{2}=6.99 \mathrm{~mm}, \varepsilon_{r_{2}}=1$.

5.15 Convergence study: Resonant frequencies as a function of the number of basis functions $(\mathrm{Q})$ used by the MoM. Rectangular cavity dimensions: $a=$ $25.4 \mathrm{~mm}, b=25.4 \mathrm{~mm}, c=23.368 \mathrm{~mm}$; DR: $h_{1}=5.54 \mathrm{~mm}, d=16.61 \mathrm{~mm}$, $\varepsilon_{r_{1}}=50$; DR support: $h_{2}=6.99 \mathrm{~mm}, \varepsilon_{r_{2}}=1$.

5.16 Convergence study: Resonant frequencies as a function of the number of basis functions (Q) used by the MoM. Rectangular cavity dimensions: $a=$ $25.4 \mathrm{~mm}, b=25.4 \mathrm{~mm}, c=23.368 \mathrm{~mm}$; DR: $h_{1}=8.4 \mathrm{~mm}, d=16.8 \mathrm{~mm}$, $\varepsilon_{r_{1}}=38$; DR support: $h_{2}=6.99 \mathrm{~mm}, \varepsilon_{r_{2}}=1 . \ldots \ldots \ldots$

5.17 Convergence study: Resonant frequencies as a function of the number of basis functions $(\mathrm{Q})$ used by the MoM. Rectangular cavity dimensions: $a=$ $25.4 \mathrm{~mm}, b=25.4 \mathrm{~mm}, c=23.368 \mathrm{~mm}$; DR: $h_{1}=8.4 \mathrm{~mm}, d=16.8 \mathrm{~mm}$, $\varepsilon_{r_{1}}=50$; DR support: $h_{2}=6.99 \mathrm{~mm}, \varepsilon_{r_{2}}=1 . \ldots \ldots \ldots$

6.1 FEST3D GUI screenshot: A four-pole DR filter is simulated. The network circuit is represented in the cambas, while the real geometry is visualized. The electromagnetic response is shown by menas of the gnuplot program.

6.2 Dialog windows to introduce the data corresponding to the dielectric-loaded rectangular cavity. . . . . . . . . . . . . . .

6.3 Bandpass structure using dielectric resonators in a rectangular waveguide enclosure. . . . . . . . . . . . . . . . . .

6.4 Electromagnetic response of the bandpass prototype described in Table 6.1. The results are compared with the FEM electromagnetic solver Ansoft HFSS. 140

6.5 Four-pole band-pass filter based on dielectric resonators in rectangular cavities connected through rectangular irises. . . . . . . . . . . . . .

6.6 Electromagnetic response of the bandpass filter described in Table 6.2. Comparison between the state space-integral equation method and the FiniteElement Method (Ansoft HFSS).

6.7 Electromagnetic response of the band-pass filter described in Table 6.2. Comparison of the scattering parameters phases between the state-space integralequation method and Ansoft HFSS.

6.8 Electromagnetic response of the band-pass filter described in Table 6.2. Comparison in the out of band range between the state-space integral-equation method and Ansoft HFSS. . . . . . . . . . . . . . . . .

6.9 Electromagnetic response of the bandpass filter described in Table 6.3. Comparison between the state space-integral equation method and the FiniteElement Method (Ansoft HFSS).

6.10 Electromagnetic response of the bandpass filter described in Table 6.4. Comparison between the state space-integral equation method and the FiniteElement Method (Ansoft HFSS). 
6.11 Bandpass filter in rectangular waveguide. The dielectric resonators are directly coupled. . . . . . . . . . . . . . . . . . . . . . . 147

6.12 Electromagnetic response of the dielectric-loaded rectangular waveguide bandpass filter described in Table 6.5 . . . . . . . . . . . . . . . . . . 148

6.13 Electromagnetic response of the dielectric-loaded rectangular waveguide bandpass filter described in Table 6.6. . . . . . . . . . . . . . . . . . . . . 149

6.14 Electromagnetic response of the dielectric-loaded rectangular waveguide bandpass filter described in Table 6.7 . . . . . . . . . . . . . . . . . . 150

6.15 Bandstop structures using dielectric resonators in rectangular waveguides. . 150

6.16 Electromagnetic response of the band-stop prototypes. Comparison between the results obtained with the state-space integral-equation method and with Ansoft HFSS. . . . . . . . . . . . . . . . . . . . . 151

6.17 Bandstop filter described in Table 6.9. . . . . . . . . . . . . . . . . 151

6.18 Electromagnetic response of the bandstop filter described in Table 6.9. . . . 152

A.1 Rectangular cavity resonator with dimensions $l_{x} \times l_{y} \times l_{z} \ldots \ldots \ldots$

D.1 Graphical representation of the domain of integration $S_{\rho, \theta}$ associated to the variables $\rho$ and $\theta$. The regions in which $S_{\rho, \theta}$ is divided to calculate numerically the integral are also represented. . . . . . . . . . . . . . 176 


\section{Table Index}

3.1 Definition of the Matrices Used in the Method of Moments . . . . . . . .

4.1 Basis Functions Used in the Method of Moments . . . . . . . . . . . . . . 57

5.1 Dimensions of the test cases extracted from [30]. Rectangular cavity dimensions: $a=25.4 \mathrm{~mm}, b=25.4 \mathrm{~mm}, c=23.37 \mathrm{~mm}$; DR relative permittivity: $\varepsilon_{r_{1}}=38$; DR support height and relative permittivity: $h_{2}=6.99 \mathrm{~mm}, \varepsilon_{r_{2}}=1.109$

5.2 Resonant frequencies of the $H E_{12}$ odd mode for the cases presented in Table 5.1. Comparison between measurements [30] and different numerical algorithms: SS-IE formulation developed in the present thesis, the Mode Matching (MM) technique [30], Finite Difference Time-Domain (FDTD) [44] and Finite Difference Frequency-Domain (FDFD) [45] techniques.

5.3 Comparison between HFSS and the new SS-IE Formulation: First three resonant modes of the structures presented in Table 5.1. . . . . . . . . .

5.4 Comparison between Ansoft HFSS and the new SS-IE formulation. Resonant frequencies $(\mathrm{GHz})$ of the first 10 modes of a dielectric-loaded resonator cavity: $a=36 \mathrm{~mm}, b=30 \mathrm{~mm}, c=40 \mathrm{~mm}$; resonator: $h_{1}=10 \mathrm{~mm}$, $d=30 \mathrm{~mm}, \varepsilon_{r_{1}}=45$; support: $h_{2}=7.5 \mathrm{~mm}, \varepsilon_{r_{2}}=1 . \ldots . . .$.

5.5 Comparison between Ansoft HFSS and the new SS-IE formulation. Resonant frequencies $(\mathrm{GHz})$ of the first 50 modes of a dielectric-loaded resonator cavity: $a=25.4 \mathrm{~mm}, b=25.4 \mathrm{~mm}, c=23.368 \mathrm{~mm}$; resonator: $h_{1}=8.4 \mathrm{~mm}, d=16.8 \mathrm{~mm}, \varepsilon_{r_{1}}=50$; support: $h_{2}=6.985 \mathrm{~mm}, \varepsilon_{r_{2}}=1$. .

5.6 Comparison between Ansoft HFSS and the SS-IE formulation. Resonant frequencies of the first 40 modes $(\mathrm{GHz}): a=30 \mathrm{~mm}, b=25.4 \mathrm{~mm}, c=$ $40 \mathrm{~mm}$; resonator: $h_{1}=6.43 \mathrm{~mm}, d_{1}=18 \mathrm{~mm}, \varepsilon_{r_{1}}=50$; support: $h_{2}=$ $11 m m, \varepsilon_{r_{2}}=1 \ldots \ldots \ldots \ldots \ldots \ldots$

6.1 Bandpass prototype parameters . . . . . . . . . . . . . . . . . . 140

6.2 Dimensions of a four-pole filter centered at $8.94 \mathrm{GHz}$. It is based on dielectricloaded cavities coupled through rectangular irises. The dielectric support of the resonators is assumed to have a relative permittivity $\varepsilon_{r_{2}}=1$, and a height of $h_{2}=3.25 \mathrm{~mm}$ 
6.3 Dimensions of a four-pole filter centered at 5.0 GHz. It is based on dielectricloaded cavities coupled through rectangular irises. The dielectric support of the resonators is assumed to have a relative permittivity $\varepsilon_{r_{2}}=1$, and a height of $h_{2}=6.73 \mathrm{~mm} \ldots \ldots \ldots \ldots \ldots \ldots$

6.4 Dimensions of a four-pole filter centered at $10.74 \mathrm{GHz}$. It is based on dielectric-loaded cavities coupled through rectangular irises. The dielectric support of the resonators is assumed to have a relative permittivity $\varepsilon_{r_{2}}=1$, and a height of $h_{2}=2.975 \mathrm{~mm} . \ldots \ldots \ldots \ldots$

6.5 Dimensions of a four-pole filter at $11.98 \mathrm{GHz}$ based on dielectric-loaded rectangular waveguide below cutoff. The dielectric support of the resonators is assumed to have a relative permittivity $\varepsilon_{r_{2}}=1$, and a height of $h_{2}=3.59$ mm. . . . . . . . . . . . . . . . . .

6.6 Dimensions of a four-pole filter at $9 \mathrm{GHz}$ based on dielectric-loaded rectangular waveguide below cutoff. The dielectric support of the resonators is assumed to have a relative permittivity $\varepsilon_{r_{2}}=1$, and a height of $h_{2}=4.96 \mathrm{~mm} .147$

6.7 Dimensions of a four-pole filter at $3.99 \mathrm{GHz}$ based on dielectric-loaded rectangular waveguide below cutoff. The dielectric support of the resonators is assumed to have a relative permittivity $\varepsilon_{r_{2}}=1$, and a height of $h_{2}=10.98$ mm. . . . . . . . . . . . . . . . . . . 147

6.8 Bandstop prototypes parameters $\ldots \ldots \ldots \ldots \ldots$

6.9 Dimensions of the bandstop filter based on dielectric-loaded cavities. The dielectric support of the resonators is assumed to have a relative permittivity $\varepsilon_{r_{2}}=1$, and a height of $h_{2}=3.25 \mathrm{~mm}$ 


\section{Chapter 1}

\section{Introduction}

\subsection{Motivation and state of the art}

During the last three decades, dielectric resonators have become a key element in many filtering applications [1-4]. Indeed, dielectric-loaded waveguide filters are frequently found in satellite and mobile communication systems, due to their advantages in terms of mass and volume reduction, low losses, and thermal stability. In the terrestrial mobile communication system industry [5,6], the cost of individual filters and the issue of mass production are crucial, whereas volume and weight are critical in satellite communications [7].

In 1939, R. D. Richtmyer [8] introduced the term dielectric resonator (DR). He showed that unmetallized dielectric objects can function as electrical resonators. Nevertheless, the first activities on dielectric resonators did not occurred until the early 1960's, when Okaya and Barash rediscovered DRs while working on high dielectric materials [9]. They provided the first analysis of modes and resonator design. During that decade, considerable efforts were devoted to the analysis of dielectric resonators [10-12]. In this area, the most notable work was developed by Cohn and his co-workers, who performed an extensive theoretical and experimental study of DR $[13,14]$. However, at that time, the lack of suitable materials (due to their poor thermal stability) made impossible the development of practical components.

The breakthrough in ceramic materials technology occurred in the early 1970's when Masse and Pucel [15] developed the first temperature-stable and low-loss barium-tetratitanate ceramic $\left(\mathrm{BaTi}_{4} \mathrm{O}_{9}\right)$. They published the design of a microstrip bandpass filter using dielectric resonators made of this ceramic [16]. In subsequent years, new improved ceramic materials were developed which made possible to use DRs for microwave applications. In 1975, the first practical DR loaded microwave filter was reported by K. Wakino [17]. The advances in ceramic technology revived interest in dielectric resonators for a wide variety of microwave circuits $[18,19]$. New theoretical work and the use of DRs to design microwave filters expanded significantly during the 1980's. In 1982, a dual-mode axially-mounted dielectric resonator was reported by S. J. Fiedziuszko [20]. It nearly matched the performance of conventional dual-mode filters and set the scene for the potential use of dielectric loaded 
multiplexers for space application. This kind of filter has been successfully used in satellite communications applications. As a consequence, complete studies on the DR's modal chart and the first rigorous full-wave analysis methods emerged in the 1980's [21-23].

In addition, the new communication systems demanding more stringent filter characteristics, have caused a significant progress in DR filter technology during the last two decades [4-6]. In particular, the rapid expansion of wireless communication industry has increased the demands for high-performance microwave filters and diplexers for both handsets and base station applications. In this area, coaxial cavity filters are commonly used due to their low cost and their spurious-free performance. Nevertheless, this kind of filters have limited quality factor values and thus a different technology must be employed to match the new filtering requirements. In this context, the high-Q dielectric resonator filters have emerged as the baseline design for wireless base stations.

Due to all these factors, the full-wave analysis and design of DR filters has deserved considerable attention in the technical literature. Indeed, the need for an accurate determination of the resonant frequencies and the related field patterns of dielectric resonator modes, together with the mathematical complexity required for solving this kind of structures, have encouraged the emergence of many different approaches for the electromagnetic analysis of this problem [19].

In the 1960's and 1970's, the use of simplified mathematical models, by introducing magnetic walls $[10,11,13,14]$ or by using other approximations $[24,25]$, allowed to obtain the electromagnetic solution for the fundamental mode of cylindrical dielectric resonators. Van Bladel and others $[26,27]$ made use of the perturbational-asymptotic technique to analyse high-permittivity isolated cylindrical and ring resonators.

In the 1980's more sophisticated methods arose. The Mode-Matching (M-M) technique was employed firstly by Kobayashi [21] to analyse a cylindrical DR enclosed in a cylindrical metal cavity. Kobayashi used such approach to determine the optimum dimension for obtaining the best separation of the spurious modes from the desired mode in pillbox and ring resonators [28]. A different approach of the M-M technique was also applied by Zaki and Atia [22], as well as by Zaki and Chen [23], to determine the modal chart of cylindrical DRs placed symmetrically in a cylindrical cavity. That work was also extended to ring resonators enclosed by cylindrical cavities [29], and to pillbox DRs placed symmetrically in rectangular cavities [30]. In subsequent years, it was used to design mixed modes dielectric resonators filters with rectangular cavities [31] and dielectric combine filters [32].

Other authors have used the orthogonal expansion method to analyse cylindrical dielectric resonators in rectangular and cylindrical cavities [33], and inhomogeneously dielectric filled cavities [34], as well as structures containing asymmetrically located, partial-height or multilayer inhomogeneous cylindrical DRs [35]. A large variety of investigations about dielectric resonators using the Finite-Element method (FEM) [36-40] can be also found in the literature, as well as Finite Difference approaches in the time-domain (FDTD) [41-44] and in the frequency-domain (FDFD) [45]. Nevertheless, the use of this kind of segmentation 
methods to deal with these problems typically require high computational resources.

Another kind of methods that have been widely used to analyse dielectric resonators are those based on the integral equation approach solved via the Method of Moments (MoM) [46]. These techniques are based on the solution of an integral equation, rather than a differential one. The integral equation is derived by means of a suitable Green's function, which constitutes the kernel. Once the integral equation is formulated, the Method of Moments is employed to test the equation and transform it into linear algebraic equations that can be solved numerically. We can differentiate basically between two possible integral formulations [47]: the Volume Integral Equation Formulation (VIEF) and the Surface Integral Equation Formulation (SIEF). In the former method, the dielectric resonator is replaced by the equivalent volumetric polarization currents [48]. In the second technique, the surface equivalence principle [49] permits to replace the DR by equivalent electric and magnetic currents on the surface defined by the interface between the dielectric resonator and the homogeneous medium in which it is immersed. In this case, the dielectric body must be homogeneous. The main difficulty of this kind of formulations lies in the ability to find the proper Green's function to solve each problem. Many authors have used this kind of approaches to determine the modal chart of DRs in free space or enclosed within metal cavities. Glisson et al. [50,51] used the surface integral equation formulation for bodies of revolution to compute the lowest resonant modes of isolated cylindrical dielectric resonators (including their field pattern). They used the free-space Green's function to obtain the integral equation, and applying the MoM obtained the resonant frequencies by searching the zeros of a matrix determinant. Omar and Schünemann [52] presented a theoretical adaptation of both, the volume and the surface integral equation formulations, to analyse DRs inside homogeneously filled waveguides. They proposed to use the dyadic Green' function for the waveguides instead of the free-space Green's function. Hanson [53] used the VIEF to describe rectangular and cylindrical dielectric resonators in free space and in microwave integrated circuits (MIC's), while Kajfez and others [54] presented a SIEF to analyse axisymmetric cavities loaded with DRs. More recently, F. Arndt [55,56] proposed a new surface integral equation formulation to analyse arbitrarily shaped dielectric resonators inside conventional rectangular cavities and rectangular cavities with rounded corners. F. Arndt utilizes the Rao-Wilton-Glisson basis functions [57] and an adaptive triangular mesh to expand the magnetic and electric currents on the surface of the dielectric resonators. Most of these approaches, however, have a common factor: the use of the free space Green's function. This fact obliges to enforce the boundary conditions on the metallic walls of the shield, thus increasing the number of unknowns of the problem. Nevertheless, this drawback can be avoided by using the dyadic Green's function of the empty cavity that encloses the dielectric resonator. F. Alessandri and others [58] have used the dyadic Green's function of the rectangular cavity to analyse DR filters in rectangular waveguides.

Moreover, most of the numerical techniques mentioned above are based on frequency- or time-domain procedures $[47,59]$. In time-domain methods, such as the finite-difference timedomain (FDTD) or the transmission-line matrix (TLM) algorithm, the temporal evolution of the field (time-step by time-step) is computed by discretizing the Maxwell's equations both 
in time and space. In a frequency-domain method, such as the finite-element method (FEM) and boundary element methods (integral equation techniques or mode matching method), the Maxwell's equations are written in the frequency domain and solved to obtain the field in the solution region computed at a set frequency. In any case, by making use of this kind of procedures, problems must be solved over and over again to find the frequency response in a broad bandwith. This drawback can be avoided using $s$-domain procedures ( $s$ is the Laplace variable).

In this sense, it is remarkable that, from the 1990's up to nowadays, several studies have been devoted to the development of new electromagnetic solvers for the analysis of passive microwave devices through state space-domain methods [60,61]. These mathematical models permit to represent a linear system described by partial differential equations in terms of an infinite poles and zeros expansion. In other words, one can obtain the electromagnetic response of a microwave device in terms of some circuit generalized matrix (scattering, admittance, impedance, etc.) represented in the form of pole expansion in the Laplace variable $s$. $S$-domain methods present several advantages: since the system is linear, there is no need to compute over and over again by stepping through time or frequency, and thus, frequency sweeps are computed very fast. Furthermore, $s$-domain solutions may be cast into equivalent electrical circuits, which is crucial in synthesis and design processes. In the case of distributed circuits, finite difference (FD) and finite element (FE) methods applied to the Maxwell equations in the $s$-domain yield to equations in which the discretized field is represented by the state variables. However, these methods need to perform refined 3D meshes, and consequently they make use of huge computational resources, slowing down significantly the numerical computations performed, specially when complex 3D structures are analysed. On the other hand, boundary element methods (BEM) avoid 3D meshes, but do not give rise directly to mathematical models in the form of pole expansions valid on a very wide bands, though fast frequency sweeps can be achieved by the adaptive Lanczos-Padè algorithm $[60,61]$.

In the work presented in this $\mathrm{PhD}$ Thesis, the generalized admittance matrix (GAM), which characterise the electromagnetic response of a dielectric-loaded cavity resonator, is obtained in terms of a pole expansion in the $s$-domain by means of a new State-Space Integral-Equation (SS-IE) approach. This technique is based in the well-known 'Boundary -Integral Resonant Mode Expansion' (BI-RME) method developed in the University of Pavia [62], which avoids frequency-per-frequency intensive computations, resulting in a very efficient algorithm.

The BI-RME method was formerly applied to obtain the normalized resonant modes of arbitrarily shaped metallic cavities in [63]. The unknown current flowing on the cavity walls was considered inside a spherical resonator, rather than in free space as it is used to be done in boundary element methods. Thus, by using the Green's functions of the spherical resonator instead of the free space one, the problem was cast by the Method of Moments $(\mathrm{MoM})$ into a real matrix linear eigenvalue problem. The same approach had been used some years before to obtain the modes of arbitrarily shaped metallic waveguides [64], which 
was further enhanced in [65].

Making use of the Kurokawa's representation of the field in a cavity resonator, it is possible to relate the pole expansion of the admittance parameters to the resonant modes of the cavity obtained by closing the structure ports with conducting planes. Following this procedure, a very efficient algorithm was developed in [62,66-68] to obtain the pole expansion of the admittance matrix based on the BI-RME method and the use of very simple expressions to represent the low-frequency behaviour of the GAM. However, the low-frequency approximation used in those works introduced a limitation in the algorithm, since the ports of the structure had to be long enough to separate the waveguides connected to the discontinuities. In subsequent years, new procedures were developed in order to overcome this drawback. In [69-71], the generalized admittance matrix of 2D and 3D structures was obtained by using the BI-RME method to obtain the resonant modes, whereas a different technique was used to find the quasi-static approximation of the GAM. It is remarkable that the proper combination of the generalized admittance matrices that characterize different building blocks in which a whole circuit is subdivided, can yield to a GAM in the form of a pole expansion that characterize the whole structure [72].

It was in [73] where a unified algorithm based only in the BI-RME method was definitively presented. In that work, the pole expansion of the GAM in the $s$-domain was directly derived from the BI-RME method. Furthermore, the meshing procedure used by this method is only performed on the boundary (2-D), and the number of unknowns involved in the problem when analysing a 3D structures is reduced drastically compared to those needed by FD or FE procedures. This approach has been applied to analyse different 3D passive microwave metallic structures demonstrating to be very efficient [74,75]. However, this method has been seldom applied to problems including dielectric obstacles, such as the full-wave analysis of H-plane filters with dielectric resonators [76]. Nowadays, in parallel to the work presented in this thesis, the research group of the University of Pavia has been also developing a different formulation to analyse rectangular waveguides loaded with dielectric resonators [77-79].

In this context, the work performed in this $\mathrm{PhD}$ Thesis is presented: a new State-Space Integral-Equation approach to analyse dielectric-loaded resonator cavities. The SS-IE formulation is based on the BI-RME method. The electromagnetic behaviour of rectangular cavities loaded with dielectric resonators is obtained through the solution of a volume integral equation. The dielectric resonator is rigorously characterized by means of the electric equivalent polarization charge and current densities defined in the volume of the dielectric object. Moreover, the kernel of the integral equation is calculated making use of the rectangular cavity Green's functions (by means of the Ewald technique), thus avoiding the introduction of additional unknowns to impose the boundary conditions on the walls of the rectangular cavity. Following an analogous procedure to the one reported in [73], the resonant modes and the field pattern of the considered cavities are obtained through the solution of a linear matrix eigenvalue problem, while the pole expansion of the generalized admittance matrix of the structure is obtained in the domain of the Laplace variable. Hence, the electromagnetic response of metal cavities loaded with dielectric resonators can be solved in a wide and dense frequency range with a very reduced computational effort. 


\subsection{Objectives}

The main objective of the work proposed in this $\mathrm{PhD}$ Thesis is the development of a new SSIE formulation to rigorously characterize the full-wave electromagnetic response of a cavity resonator loaded with a dielectric inset. For this purpose, the generalized admittance matrix (GAM) that represent the cavity resonator is accurately calculated. This technique has been implemented to analyse rectangular cavities loaded with a cylindrical dielectric resonator. The efficient analysis of this building block by means of the SS-IE approach is a key issue, since the dielectric resonator characterization requires the major part of the computational resources when one analyzes more complex structures such as dielectric resonator filters. Once the GAM that represents the building block is calculated, it may be connected to other circuit elements, in order to solve different typologies of dielectric resonator filters.

Thus, the main objectives of the presented work are the following ones:

1. Extension of the BI-RME method to calculate the modal chart of a cavity resonator loaded with a linear, homogeneous and isotropic dielectric inset. A perfect conductive walls resonant cavity is considered, including a dielectric inset placed at any arbitrary position inside the cavity. The resonant frequencies are calculated by means of a linear matrix eigenvalue problem as well as the electromagnetic field distribution.

2. Application of the new formulation to calculate the modal chart of a rectangular cavity loaded with a cylindrical dielectric resonator placed at an arbitrary position inside the cavity. The well knowledge of the electromagnetic field distribution is important to properly understand the coupling mechanism of the resonant cavity to adjacent circuit elements, such as irises, ports or other resonant cavities. The software tool developed has been validated with technical literature and the well-known commercial software Ansoft HFSS [80].

3. Formulation of a new state-space integral-equation (SS-IE) approach to calculate the generalized admittance matrix of a resonant cavity loaded with a linear, homogeneous and isotropic dielectric inset. As described above, the GAM is obtained as a pole expansion in the domain of the Laplace variable $s$.

4. Application of the new SS-IE formulation to calculate the GAM of a rectangular cavity loaded with a cylindrical dielectric resonator placed at an arbitrary position inside the cavity. To calculate the GAM, the access apertures in the cavity are represented by magnetic currents, and the implementation has been performed in two different phases:

- Aperture of two parallel access ports.

- Aperture of the four access ports in the lateral cavity sides.

5. Integration of the software module into FEST3D [81]. The new software tool allows us to characterize the electromagnetic response of the resonant cavity, as well as to connect it to other circuit elements, in order to consider more complex structures. This 
software module has been integrated in a Computer Aided Engineering tool (CAE tool) for the analysis and design of passive microwave and millimeter-wave components: FEST3D. Thus, the integration into such a general purpose CAE tool permits us to analyze and design different bandpass and stopband filters that have been used to fully validate the proposed algorithm.

6. Finally, different topologies of dielectric resonator filters in waveguide technology have been studied in order to design bandpass and stopband filters. The designed filters have been used to validate the method developed comparing the results obtained with Ansoft HFSS.

\subsection{Thesis structure}

This PhD Thesis is composed of seven chapters. The first chapter is the present one, which is the introduction. A brief review of the state of the art on the field of dielectric resonator filters for microwave applications and numerical techniques to analyse the electromagnetic behaviour of this kind of components have been presented. The main objectives of the present work have been detailed as well.

In Chapter 2, the problem of determining the electromagnetic field generated by electric and/or magnetic sources in cavity resonators, the so-called 'interior problem', is presented. The main objective of this chapter is to present the 'hybrid' representation of the electromagnetic field generated by electric and/or magnetic sources in an ideal cavity proposed by G. Conciauro and M. Bressan in [82]. Following this approach, the electromagnetic fields are expressed in terms of the scalar and vector potentials in the Coulomb gauge, partly as Green's integrals, and partly as resonant mode expansion. This representation permits to extract the singularities of the dyadic Green's functions in closed form, and it sets the starting point for the State-Space Integral-Equation formulation presented in Chapter 3.

In Chapter 3, the State-Space Integral-Equation (SS-IE) approach to characterise dielectricloaded resonator cavities is presented. As mentioned above, this formulation is based on the BI-RME method. As it is shown in the first section of this chapter, the dielectric resonator has been rigorously characterized by means of the electric equivalent polarization charge and current densities defined in the volume of the dielectric object. Starting from the 'hybrid' representation of the field in a cavity resonator presented in Chapter 2, the SS-IE formulation is developed. Following this technique, the resonant modes of the considered cavities are obtained through the solution of a linear matrix eigenvalue problem. Finally, in the last section of this chapter, the pole expansion of the generalized admittance matrix of the dielectric-loaded cavity is obtained in the domain of the Laplace variable.

The formulation presented in Chapter 3 is applied to characterize the electromagnetic response of a rectangular cavity loaded with a cylindrical dielectric resonator in Chapter 4 . In this chapter, we describe the basis functions used to approximate the polarization vector that characterize the dielectric object inside the cavity. Throughout this chapter we also show 
how crucial is the selection of an appropriate set of basis functions, not only for the good convergence of the method proposed, but also to transform some of the integrals involved in the problem. In the third section of Chapter 4 we explain how we have calculated the entries of the matrices involved in the solution of the problem. As it is shown, the numerical efficiency of the proposed method depends critically on the computation of these matrices, and specially of those matrices involving the Green's functions.

In Chapter 5, we present the electromagnetic analysis of different rectangular cavities loaded with a dielectric puck using the SS-IE formulation previously derived. The resonant frequencies of low and high order modes are calculated, as well as their electromagnetic field distributions. The influence of geometrical and electric parameters in the computation of the modal chart of the cavity resonator is studied. The results obtained with the new developed formulation are successfully compared with technical literature and with the well-known commercial tool Ansoft High Frequency Structure Simulator (Ansoft HFSS) [80], which is based on the Finite-Element Method (FEM). The fact that the algorithm used by HFSS is completely different from the SS-IE formulation presented in this work, makes it a good reference to validate the software developed in this thesis. This study serves us not only to perform a first validation of the method presented, but also to understand the electromagnetic behaviour of the dielectric-loaded rectangular cavities under analysis.

In Chapter 6, different single-mode DR filters are presented. We have implemented a software module to characterise the circuit building block represented by a rectangular cavity loaded with a cylindrical dielectric resonator. The resonator cavity may be opened through any of its lateral access ports and/or through its top surface. Once the generalized admittance matrix of this circuit building block is calculated, it may be connected to other circuit elements, in order to analyse different typologies of dielectric resonator filters. For such a purpose, we have integrated this software module in a Computer Aided Engineering (CAE) tool for the analysis and design of passive microwave and millimetre-waves components: FEST3D (Full-wave Electromagnetic Simulation Tool 3D) [81]. In this chapter, we give a brief introduction to the modal electromagnetic solver FEST3D. We also describe how the algorithm developed in this $\mathrm{PhD}$ Thesis has been integrated into FEST3D. Finally, different single-mode dielectric resonator bandpass filters, as well as a stopband filter, are presented. The bandpass filters may be designed by using dielectric-loaded rectangular cavities coupled through irises, or by placing the dielectric resonators in a rectangular waveguide below cutoff. The results obtained by using the SS-IE approach integrated into FEST 3D are successfully compared with those obtained by the commercial software tool Ansoft HFSS [80].

Finally, in Chapter 7 the conclusions are presented. Moreover, some guide lines for future research activities in the field are proposed as a consequence of the work performed in this PhD Thesis. 


\section{Chapter 2}

\section{Theory of Cavity Resonators Review}

\subsection{Introduction}

A cavity resonator is a metallic enclosure including a lossless (or low-loss) medium, in which modes of free oscillation can exist at an infinite number of discrete frequencies. The cavity usually communicates with the outside through apertures providing coupling to a waveguide or by means of a probe/loop that terminates in a coaxial transmission line. The electromagnetic field is generated by internal sources and/or transmitted into the cavity through these apertures or coaxial excitations.

The determination of the electromagnetic field generated by electric and/or magnetic sources in cavity resonators, i. e., inside a finite volume under given boundary conditions, represents a fundamental problem in electromagnetism. This is the so-called 'interior problem'.

The first complete theory of the cavity resonators containing isotropic and homogeneous media was presented by Kurokawa in 1958 [83], and it was reported in many books in subsequents years [84-86]. Kurokawa proposed an orthonormal base, consisting of both irrotational and solenoidal modes, for the electromagnetic field expansion. This way, expanding the electromagnetic fields in terms of these complete orthonormal basis functions, the problem of solving the Maxwell's equation in a cavity can be reduced to that of determining the expansion coefficients. The solution of this problem yields to a general representation of the electromagnetic field in the form of a pole expansion in the frequency domain in terms of the resonant modes of the cavity resonator.

The electromagnetic field inside a cavity resonator can be also expressed in its integral form by means of the Green's functions. Indeed, due to the existence of internal sources, the interior problem is directly related to the problem of calculating the electromagnetic field in the source region. In this case, the behaviour of the electric dyadic Green's function $\left(G_{e}\right)$ needed to describe the field is extremely singular at the source region, and thus it is necessary to calculate it efficiently. Many authors have determined the electric dyadic Green's function in form of eigenfunction series [87-89], but these representations are inadequate due to their poor convergence properties near the singularities of $\underline{G}_{e}$. This drawback was overcome in 
the work presented by M. Bressan and G. Conciauro in 1983 [82]. They expressed $\underline{G}_{e}$ as a sum of one irrotational and two solenoidal terms, and evidenced the singularities of the irrotational term and the former solenoidal one in a closed form. Following this approach, the remaining terms containing the eigenfunction series were demonstrated to converge rapidly. Furthermore the authors pointed out that the use of this formulation yields to an 'hybrid' representation of the field in terms of the scalar and vector potentials in the Coulomb gauge. Indeed, it is considered an 'hybrid' representation because the electromagnetic field is finally expressed partly as Green's integrals, and partly as resonant mode expansion. This technique has been widely applied in subsequent works $[63,71,73-75]$ and it is detailed in the book [62].

The main objective of this chapter is to present the hybrid representation of the electromagnetic field generated by electric and/or magnetic sources in an ideal cavity. This topic will set the starting point for further discussion about the formulation presented in chapter 3 . For this purpose, the second and third sections of this chapter summarize the main results of the Kurokawa's theory of cavity resonators. The fourth section reproduces the main results of the approach followed in [82] for the efficient calculation of the electric dyadic Green's function. Finally, the last three sections connect the results obtained in the previous ones in order to provide the 'hybrid' representation of the electromagnetic field used in this Thesis.

\subsection{The expansion of electromagnetic fields in cavity res- onators}

The theory of cavity resonators may be applied to different types of cavity (see Fig. 2.1). However, in this section we will focus our attention in simply connected cavities with single boundary (for multi-connected cavities or simply connected with double boundary see [62]).

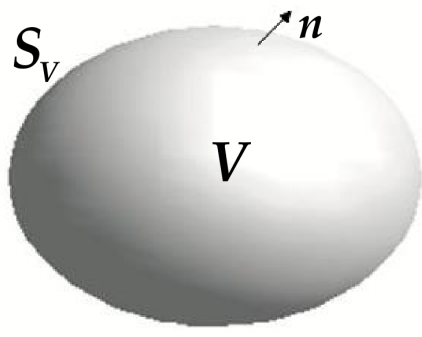

(a) Simply connected with simple boundary

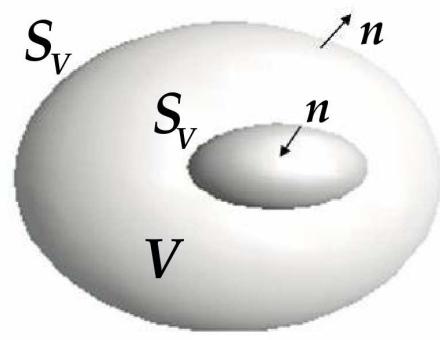

(b) Simply connected with double boundary

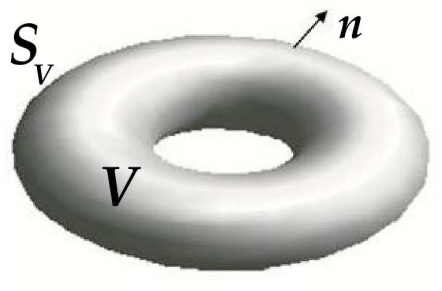

(c) Multi-connected with simple boundary

Figure 2.1: Different types of cavity resonators.

Due to the finiteness of the electromagnetic energy stored in a finite volume, the electric and magnetic fields belong to the space $\mathcal{L}_{2}(V)$, made up of complex square-integrable vector 
fields defined in the cavity volume $V$. The inner product of this space is defined as [62]:

$$
\langle\vec{a}, \vec{b}\rangle=\int_{V} \vec{a}^{*} \cdot \vec{b} d v \quad \vec{a}, \vec{b} \in \mathcal{L}_{2}(V)
$$

where $*$ denotes complex conjugate.

The theory of cavity resonators is based on the possibility of representing the electromagnetic field using an appropriate orthonormal bases of $\mathcal{L}_{2}(V)$. The bases are the electric and magnetic eigenvectors. The electric eigenvectors are the solution of the eigenvalue problem:

$$
\begin{aligned}
& \nabla^{2} \vec{V}+\Lambda \vec{V}=0 \quad \text { in } \quad V \\
& \left.\begin{array}{rl}
\nabla \cdot \vec{V} & =0 \\
\widehat{n} \times \vec{V} & =0
\end{array}\right\} \quad \text { on } \quad S_{V}
\end{aligned}
$$

where $\Lambda$ is the eigenvalue. And the magnetic eigenvectors are the solution of the eigenvalue problem:

$$
\left.\begin{array}{rl}
\nabla^{2} \vec{V}+\Lambda \vec{V} & =0 \\
\widehat{n} \cdot \vec{V} & =0 \\
\widehat{n} \times \nabla \times \vec{V} & =0
\end{array}\right\} \quad \text { in } \quad \text { on } \quad S_{V}
$$

The solutions of both the electric and magnetic eigenvalue problems have the following properties:

1. there is a countable infinity of real non-negative eigenvalues and real eigenvectors;

2. eigenvalues have a single cluster point at infinity;

3. eigenvalues are simple or have a finite degree of degeneracy;

4. eigenvectors are continuously differentiable to all orders, and mutually orthogonal in $\mathcal{L}_{2}(V)$

5. assuming the normalization

$$
\left\|\vec{V}_{i}\right\|^{2}=\left\langle\vec{V}_{i}, \vec{V}_{i}\right\rangle=1
$$

the eigenvector set constitutes an orthonormal basis of $\mathcal{L}_{2}(V)$; therefore, any squareintegrable vector field $\vec{a}$, defined in $V$, can be represented by the expansion:

$$
\vec{a}=\sum_{i}^{\infty}\left\langle\vec{V}_{i}, \vec{a}\right\rangle \vec{V}_{i}
$$

According to the Helmholtz's theorem, the set of both the electric and magnetic eigenvectors can be divided into solenoidal and irrotational eigenvectors. In case of separate boundaries or multi-connected cavities, the eigenvectors can also be divided into harmonic ones [62]. 


\subsubsection{Electric eigenvectors}

IRROTATIONAL EIGENVECTORS - These eigenvectors are denoted as $\vec{f}_{1}, \vec{f}_{2}, \ldots, \vec{f}_{i}, \ldots$ being its corresponding eigenvalues $\mu_{1}^{2}, \mu_{2}^{2}, \ldots, \mu_{i}^{2}, \ldots$, respectively. By definition, the irrotational eigenvectors satisfy:

$$
\nabla \times \vec{f}_{i}=0, \quad \nabla \cdot \vec{f}_{i} \neq 0 \quad \text { in } \quad V
$$

and are the solution of:

$$
\left(\nabla^{2}+\mu_{i}^{2}\right) \vec{f}_{i}=0 \quad \text { in } \quad V
$$

The irrotational modes are generated from the scalar functions $\phi_{i}$ that are solutions of

$$
\begin{aligned}
\nabla^{2} \phi_{i}+\mu_{i}^{2} \phi_{i}=0 & \text { in } \quad V \\
\phi_{i}=0 & \text { on } \quad S_{V}
\end{aligned}
$$

from which it can be obtained that

$$
\overrightarrow{f_{i}}=\frac{\nabla \phi_{i}}{\mu_{i}}
$$

Apart from these properties, it can be demonstrated that we can always construct a set of orthonormal irrotational eigenvectors. Making use of the equations (2.4b), (2.5) and the vector identity,

$$
\nabla \cdot\left(\phi_{i} \nabla \phi_{i}\right)=\phi_{i} \nabla^{2} \phi_{i}+\nabla \phi_{i} \cdot \nabla \phi_{i}
$$

it can be demonstrated that if $\phi_{i}$ is normalized then $\vec{f}_{i}$ is also normalized:

$$
\int_{V} \vec{f}_{i} \cdot \vec{f}_{i} d v=\int_{V} \phi_{i}^{2} d v-\frac{1}{\mu_{i}^{2}} \int_{S_{V}} \phi_{i} \frac{\partial \phi_{i}}{\partial n} d s=\int_{V} \phi_{i}^{2} d v=1
$$

Next, we can also demonstrate the orthogonality relationship between irrotational eigenvectors. Firstly, we express the inner product between two eigenvectors as the product of its corresponding scalar functions,

$$
\int_{V} \vec{f}_{i} \cdot \vec{f}_{j} d v=\int_{V} \frac{\nabla \phi_{i}}{\mu_{i}} \cdot \frac{\nabla \phi_{j}}{\mu_{j}} d v=\frac{1}{\mu_{i} \mu_{j}} \int_{V}\left[\nabla \cdot\left(\phi_{i} \nabla \phi_{j}\right)-\phi_{i} \nabla^{2} \phi_{j}\right] d v=\frac{\mu_{j}}{\mu_{i}} \int_{V} \phi_{i} \phi_{j} d v
$$

Following now a similar procedure to the used for the expression (2.6), we can write:

$$
\begin{aligned}
\left(\mu_{i}^{2}-\mu_{j}^{2}\right) \int_{V} \phi_{i} \cdot \phi_{j} d v & =\int_{V}\left(\phi_{i} \cdot \nabla^{2} \phi_{j}-\phi_{j} \cdot \nabla^{2} \phi_{i}\right) d v= \\
& =\int_{V} \nabla \cdot\left(\phi_{i} \cdot \nabla \phi_{j}-\phi_{j} \cdot \nabla \phi_{i}\right) d v=\int_{S_{V}} \phi_{i} \frac{\partial \phi_{j}}{\partial n}-\phi_{j} \frac{\partial \phi_{i}}{\partial n} d s=0
\end{aligned}
$$


Therefore, when $\mu_{i} \neq \mu_{j}$ the eigenvectors are orthonormal, whereas for degenerate eigenvalues it can be used the Gram-Schmidt orthogonalization procedure to construct a new subset of orthonormal eigenvectors. And thus, one can always construct a set of irrotational eigenvectors that satisfy:

$$
\int_{V} \vec{f}_{i} \cdot \vec{f}_{j} d v=\delta_{i j}
$$

SOLENOIDAL EIGENVECTORS - The electric solenoidal modes are denoted as $\vec{E}_{1}$, $\vec{E}_{2}, \ldots, \vec{E}_{i}, \ldots$ and its corresponding eigenvalues as $k_{1}^{2}, k_{2}^{2}, \ldots, k_{i}^{2}, \ldots$, respectively. By definition they satisfy:

$$
\nabla \times \vec{E}_{i} \neq 0, \quad \nabla \cdot \vec{E}_{i}=0 \quad \text { in } \quad V
$$

And therefore, taking into account (2.1a), the solenoidal eigenvectors satisfy:

$$
\begin{aligned}
\nabla \times \nabla \times \vec{E}_{i}-k_{i}^{2} \vec{E}_{i}=0 & \text { in } \quad V \\
\widehat{n} \times \vec{E}_{i}=0 & \text { on } S_{V}
\end{aligned}
$$

There exist an infinity number of positive eigenvalues an real eigenvectors. Next, we can demonstrate that the solenoidal eigenvectors are mutually orthogonal. For this purpose we substract the equations (2.8b) satisfied by $\vec{E}_{i}$ and $\vec{E}_{j}$, just obtaining

$$
\vec{E}_{i} \cdot \nabla \times \nabla \times \vec{E}_{j}-\vec{E}_{j} \cdot \nabla \times \nabla \times \vec{E}_{i}=\left(k_{j}^{2}-k_{i}^{2}\right) \vec{E}_{i} \cdot \vec{E}_{j},
$$

and then we can write:

$$
\begin{aligned}
\left(k_{j}^{2}-k_{i}^{2}\right) \int_{V} \vec{E}_{i} \cdot \vec{E}_{j} d v & =\int_{V}\left(\vec{E}_{i} \cdot \nabla \times \nabla \times \vec{E}_{j}-\vec{E}_{j} \cdot \nabla \times \nabla \times \vec{E}_{i}\right) d v \\
& =\int_{V} \nabla \cdot\left(\vec{E}_{j} \times \nabla \times \vec{E}_{i}-\vec{E}_{i} \times \nabla \times \vec{E}_{j}\right) d v \\
& =\int_{S_{V}}\left(\widehat{n} \times \vec{E}_{j} \cdot \nabla \times \vec{E}_{i}-\widehat{n} \times \vec{E}_{i} \cdot \nabla \times \vec{E}_{j}\right) d s=0
\end{aligned}
$$

where we have used the vector identity $\nabla \cdot(\vec{a} \times \vec{b})=\vec{b} \cdot \nabla \times \vec{a}-\vec{a} \cdot \nabla \times \vec{b}$, and the boundary condition satisfied by the solenoidal electric eigenvectors. As a consequence, when $k_{i} \neq k_{j}$ the eigenvectors are orthonormal, whereas for degenerate eigenvalues it can be used the GramSchmidt orthogonalization procedure to construct a new subset of orthonormal eigenvectors. And thus, assuming that the $\vec{E}_{i}$ are normalized, we can write:

$$
\int_{V} \vec{E}_{i} \cdot \vec{E}_{j} d v=\delta_{i j}
$$

Furthermore, the orthogonalization between the solenoidal and the irrotational electric eigenvectors can be easily demonstrated. Since $\nabla \times \vec{f}_{i}=0$ and $\nabla \times \nabla \times \vec{E}_{j}=-k_{j}^{2} \vec{E}_{j}$, we can write:

$$
\nabla \cdot\left(\vec{f}_{i} \times \nabla \times \vec{E}_{j}\right)=\nabla \times \vec{f}_{i} \cdot \nabla \times \vec{E}_{j}-\vec{f}_{i} \cdot \nabla \times \nabla \times \vec{E}_{j}=-k_{i}^{2} \vec{f}_{i} \cdot \vec{E}_{j}
$$


And thus, we obtain:

$$
k_{j}^{2} \int_{V} \vec{f}_{i} \cdot \vec{E}_{j} d v=\int_{S_{V}} \widehat{n} \times \vec{f}_{i} \cdot \nabla \times \vec{E}_{j} d s=0
$$

\subsubsection{Magnetic eigenvectors}

IRROTATIONAL EIGENVECTORS - These eigenvectors are denoted as $\vec{g}_{1}, \vec{g}_{2}, \ldots, \vec{g}_{i}, \ldots$ and its corresponding eigenvalues as $\nu_{1}^{2}, \nu_{2}^{2}, \ldots, \nu_{i}^{2}, \ldots$, respectively. Once again, by definition, the irrotational eigenvectors satisfy:

$$
\nabla \times \vec{g}_{i}=0, \quad \nabla \cdot \vec{g}_{i} \neq 0 \quad \text { in } V
$$

and are the solution of:

$$
\left(\nabla^{2}+\nu_{i}^{2}\right) \vec{g}_{i}=0 \quad \text { in } \quad V
$$

The irrotational modes are generated from a scalar function $\psi_{i}$ that are solutions of

$$
\begin{aligned}
\nabla^{2} \psi_{i}+\nu_{i}^{2} \psi_{i} & =0 & & \text { in } \quad V \\
\frac{\partial \psi_{i}}{\partial n} & =0 & & \text { on } \quad S_{V}
\end{aligned}
$$

from which it can be obtained that

$$
\vec{g}_{i}=\frac{\nabla \psi_{i}}{\nu_{i}}
$$

And analogously to the case of the electric irrotational eigenvectors, the normalization of $g_{i}$ is implied by the normalizing condition of $\psi_{i}$ :

$$
\int_{V} \vec{g}_{i} \cdot \vec{g}_{i} d v=\int_{V}\left|\psi_{i}\right|^{2} d v=1
$$

SOLENOIDAL EIGENVECTORS - The magnetic solenoidal eigenvectors are denoted as $\vec{H}_{1}, \vec{H}_{2}, \ldots, \vec{H}_{i}, \ldots$ and its corresponding eigenvalues as $k_{1}^{2}, k_{2}^{2}, \ldots, k_{i}^{2}, \ldots$, respectively. By definition they satisfy:

$$
\nabla \times \vec{H}_{i} \neq 0 \quad \nabla \cdot \vec{H}_{i}=0
$$

And therefore, taking into account (2.2a) and (2.2b), the magnetic solenoidal eigenvectors satisfy:

$$
\begin{aligned}
\nabla \times \nabla \times \vec{H}_{i}-k_{i}^{2} \vec{H}_{i}=0 & \text { in } \quad V \\
\widehat{n} \times \nabla \times \vec{H}_{i}=0 & \text { on } S_{V}
\end{aligned}
$$


There exist an infinity number of positive eigenvalues an real eigenvectors. If we assume that the $\vec{H}_{i}$ are normalized, we can proceed like in the case of the electric eigenvectors and taking into account now the boundary conditions of $\vec{H}_{i}$ and $\vec{g}_{i}$, we can easily obtain the orthogonalization properties:

$$
\begin{gathered}
\int_{V} \vec{H}_{i} \cdot \vec{H}_{j} d v=\delta_{i j} \\
\int_{V} \vec{H}_{i} \cdot \vec{g}_{j} d v=0
\end{gathered}
$$

So far, we have used the same symbol $k_{i}^{2}$ for the eigenvalues of both the electric and magnetic solenoidal eigenvectors, and this is due to the fact that both spectra coincide. If we take the curl of (2.8b) and (2.15b), we can observe that $\nabla \times \vec{E}_{i}$ satisfies the same equations as $\vec{H}_{i}$ and that, by duality, $\nabla \times \vec{H}_{i}$ satisfies the same equations as $\vec{E}_{i}$. As a consequence, we can state that the eigenvalue spectra of the electric and magnetic solenoidal eigenvectors are the same. Moreover, it is inferred that $\nabla \times \vec{E}_{i}$ is proportional to $\vec{H}_{i}$ and $\nabla \times \vec{H}_{i}$ is proportional to $\vec{E}_{i}$. Both proportionality constants must be $\pm k_{i}$, so choosing the positive sign, we can write:

$$
\begin{aligned}
& \nabla \times \vec{H}_{i}=k_{i} \vec{E}_{i} \\
& \nabla \times \vec{E}_{i}=k_{i} \vec{H}_{i}
\end{aligned}
$$

When the eigenvectors $\vec{E}_{i}$ are normalized, then the eigenvectors $\vec{H}_{i}$ are automatically normalized. Starting from the expression

$$
\begin{aligned}
k_{i}^{2} \vec{H}_{i} \cdot \vec{H}_{i} & =k_{i}^{2} \nabla \times \vec{E}_{i} \cdot \nabla \times \vec{E}_{i}=\nabla \cdot\left(\vec{E}_{i} \times \nabla \times \vec{E}_{i}\right)+\vec{E}_{i} \cdot \nabla \times \nabla \times \vec{E}_{i} \\
& =\nabla \cdot\left(\vec{E}_{i} \times \nabla \times \vec{E}_{i}\right)+k_{i}^{2} \vec{E}_{i} \cdot \vec{E}_{i}
\end{aligned}
$$

we can integrate over the volume and using the boundary condition $\widehat{n} \times \vec{E}_{i}=0$, to finally obtain:

$$
\left\|\vec{H}_{i}\right\|^{2}=\left\|\vec{E}_{i}\right\|^{2}=1
$$

\subsection{Forced oscillations in cavity resonators}

In the preceding section we have presented a set of orthonormal eigenvectors that serves as basis to expand the electromagnetic field in a simple connected cavity with single boundary and with an isotropic and homogeneous medium. In such a space the field inside the cavity can be represented as a linear eigenvector expansion:

$$
\begin{aligned}
& \vec{E}=\sum_{i=1}^{\infty} A_{i} \vec{E}_{i}+\sum_{i=1}^{\infty} F_{i} \vec{f}_{i} \\
& \vec{H}=\sum_{i=1}^{\infty} B_{i} \vec{H}_{i}+\sum_{i=1}^{\infty} G_{i} \vec{g}_{i}
\end{aligned}
$$


The field in the cavity can be generated by interior sources or by exciting it by apertures in the enclosure and they must obey the Maxwell's equations:

$$
\begin{aligned}
\nabla \times \vec{H} & =j \omega \epsilon \vec{E}+\vec{J} \\
-\nabla \times \vec{E} & =j \omega \mu \vec{H}+\vec{M}
\end{aligned}
$$

where $\vec{J}$ and $\vec{M}$ are, respectively, the electric and magnetic current densities distributed in the cavity.

In order to obtain the expansion coefficients of the interior problem, we insert now (2.20) in (2.21). It is remarkable that the boundary conditions satisfied by the eigenvectors are not the same as the boundary conditions satisfied by the field expanded. This fact means that the series in (2.20) are no-uniform convergent, and thus the curl of the infinite sum is not equal to the sum of the curls, i. e., the above series can not be differentiated term by term [84-86]. For this reason, we will proceed to expand the Maxwell's equations (2.21) in terms of the eigenvectors.

Since $\nabla \times \vec{H}$ has the form of an electric field, we expand the equation (2.21a) in terms of the electric eigenvectors. We start projecting it in the subset of the electric irrotational eigenvectors:

$$
\int_{V} \nabla \times \vec{H} \cdot \vec{f}_{i} d v=j \omega \epsilon \int_{V} \vec{E} \cdot \vec{f}_{i} d v+\int_{V} \vec{J} \cdot \vec{f}_{i} d v
$$

Using $\nabla \times \vec{f}_{i}=0$ and the boundary condition $\widehat{n} \times \vec{f}_{i}=0$, it can be observed that the left term of equation (2.22) is equal to zero, and thus

$$
F_{i}=\int_{V} \vec{E} \cdot \vec{f}_{i} d v=j \frac{\eta}{k} \int_{V} \vec{J} \cdot \vec{f}_{i} d v
$$

where $k=\omega \sqrt{\epsilon \mu}$ is the wavenumber at the operating frequency and and $\eta=\sqrt{\frac{\mu}{\epsilon}}$ is the characteristic impedance of the medium.

We follow now the same procedure with the electric solenoidal eigenvector:

$$
\int_{V} \nabla \times \vec{H} \cdot \vec{E}_{i} d v=j \frac{k}{\eta} \int_{V} \vec{E} \cdot \vec{E}_{i} d v+\int_{V} \vec{J} \cdot \vec{E}_{i} d v
$$

Using $\nabla \times \vec{E}_{i}=k_{i} \vec{H}_{i}$ and the boundary condition $\widehat{n} \times \vec{E}_{i}=0$, we can write

$$
k_{i} B_{i}=j \frac{k}{\eta} A_{i}+\int_{V} \vec{J} \cdot \vec{E}_{i} d v
$$

Since $\nabla \times \vec{E}$ has the form of a magnetic field, we will expand now the equation (2.21b) in terms of the magnetic eigenvectors. By multiplying the expression by $\vec{g}_{i}$ and integrating over the cavity volume,

$$
\int_{V} \nabla \times \vec{E} \cdot \vec{g}_{i} d v=-j k \eta \int_{V} \vec{H} \cdot \vec{g}_{i} d v-\int_{V} \vec{M} \cdot \vec{g}_{i} d v
$$


and using

$$
\int_{V} \nabla \times \vec{E} \cdot \vec{g}_{i} d v=\int_{S_{V}} \vec{g}_{i} \cdot(\widehat{n} \times \vec{E}) d s
$$

we calculate the coefficients $G_{i}$ :

$$
G_{i}=\frac{j}{k \eta}\left(\int_{V} \vec{M} \cdot \vec{g}_{i} d v+\int_{S_{V}} \vec{g}_{i} \cdot(\widehat{n} \times \vec{E}) d s\right)
$$

Expanding analogously the expression (2.21b) in terms of the magnetic solenoidal eigenvectors $\vec{H}_{i}$, we have:

$$
k_{i} A_{i}+\int_{S_{V}} \vec{H}_{i} \cdot(\widehat{n} \times \vec{E}) d s=-j k \eta B_{i}-\int_{V} \vec{M} \cdot \vec{H}_{i} d v
$$

And thus, we can finally use the equations (2.25) and (2.28) to obtain the coefficients $A_{i}$ and $B_{i}$ :

$$
\begin{aligned}
& A_{i}=\frac{k_{i}\left(\int_{V} \vec{M} \cdot \vec{H}_{i} d v+\int_{S_{V}} \vec{H}_{i} \cdot(\widehat{n} \times \vec{E}) d s\right)+j k \eta \int_{V} \vec{J} \cdot \vec{E}_{i} d v}{k^{2}-k_{i}^{2}} \\
& B_{i}=\frac{\frac{j k}{\eta}\left(\int_{V} \vec{M} \cdot \vec{H}_{i} d v+\int_{S_{V}} \vec{H}_{i} \cdot(\widehat{n} \times \vec{E}) d s\right)-k_{i} \eta \int_{V} \vec{J} \cdot \vec{E}_{i} d v}{k^{2}-k_{i}^{2}}
\end{aligned}
$$

Therefore, expressions (2.23), (2.27), (2.29) and (2.30) give us the value of the coefficient expansions in (2.20). These equations represent the field in the form of a singularity expansion in the frequency domain. The singularities consist of a simple pole of the coefficients $F_{i}$ and $G_{i}$ at $\omega=0$, and an infinity of simple pole pairs of the coefficients $A_{i}$ and $B_{i}$, at the frequencies $\pm \omega_{i}$, where

$$
\omega_{i}=\frac{k_{i}}{\sqrt{ } \epsilon \mu}
$$

As a consequence, we can express the field generated by magnetic and/or electric sources in a cavity with perfect conducting walls $(\widehat{n} \times \vec{E}=0)$ as follows:

$$
\begin{aligned}
& \vec{E}=j \frac{\eta}{k} \sum_{i=1}^{\infty}\left\langle\vec{f}_{i}, \vec{J}\right\rangle \vec{f}_{i}+j \eta k \sum_{i=1}^{\infty} \frac{\left\langle\vec{E}_{i}, \vec{J}\right\rangle}{k^{2}-k_{i}^{2}} \vec{E}_{i}+\sum_{i=1}^{\infty} k_{i} \frac{\left\langle\vec{H}_{i}, \vec{M}\right\rangle}{k^{2}-k_{i}^{2}} \vec{E}_{i} \\
& \vec{H}=\frac{j k}{\eta} \sum_{i=1}^{\infty} \frac{\left\langle\vec{H}_{i}, \vec{M}\right\rangle}{k^{2}-k_{i}^{2}} \vec{H}_{i}-\sum_{i=1}^{\infty} \frac{k_{i}\left\langle\vec{E}_{i}, \vec{J}\right\rangle}{k^{2}-k_{i}^{2}} \vec{H}_{i}+\frac{j}{\eta k} \sum_{i=1}^{\infty}\left\langle\vec{g}_{i}, \vec{M}\right\rangle \vec{g}_{i}
\end{aligned}
$$




\subsection{Electric Green's functions in the source region. Singu- larity extraction}

As we will see in Chapter 3, when calculating the electromagnetic fields in a rectangular cavity loaded with a dielectric resonator, the electric sources $\vec{J}$ involved in the solution depend on the electric field. As a consequence, we will have to deal with the problem of computing the electric dyadic Green's function in the source region.

The behaviour of the electric dyadic Green's function is 'extremely' singular when the observation and the source points coalesce, and that makes highly difficult to calculate it accurately near or in the singularity point. Thus, we will have to pay special attention to the approach used to determine $\underline{G}_{e}$ in order to calculate efficiently the electromagnetic field generated by internal sources in a cavity resonator.

In 1961 J. Van Bladel [90] pointed out the necessity of isolating the singularity of the electric dyadic Green's function in free space to determine the field in the source region. In 1980 A. Yaghjian [91] generalized this concept for any kind of geometry providing an approach to rigorously derive a generalized electric dyadic Green's function which defines uniquely the electric field inside and outside the source region. Following that work, the electric field generated by electric sources in a cavity resonator with perfect conducting walls, filled with linear, isotropic and homogeneous medium may be expressed as:

$$
\vec{E}(\vec{r})=-j \omega \mu \lim _{\delta \rightarrow 0} \int_{V-V_{\delta}} \underline{G}_{e}\left(\vec{r}, \vec{r}^{\prime}\right) \cdot \vec{J}\left(\vec{r}^{\prime}\right) d v^{\prime}-\frac{\underline{L} \cdot \vec{J}(\vec{r})}{j \omega \epsilon}
$$

where $\vec{r}$ and $\vec{r}^{\prime}$ are, respectively, the observation and the source points, and $\underline{L}$ is the socalled depolarizing dyadic, which is a symmetric constant dyadic that depends solely on the geometry of the 'principal volume' $V_{\delta}$. The principal volume excludes the singularity of $\underline{G}_{e}$ and becomes infinitesimally small in the limit as its maximum chord length $\delta$ approaches to zero. In [91], it was demonstrated that the general expression of the depolarizing dyadic is:

$$
\underline{L}=\frac{1}{4 \pi} \int_{S_{\delta}} \frac{\vec{R} \widehat{u}_{n_{\delta}}}{R^{3}} d s^{\prime}
$$

where $S_{\delta}$ is the surface of the principal volume $V_{\delta}, \widehat{u}_{n_{\delta}}$ is the inward normal vector to $S_{\delta}$, $\vec{R}=\vec{r}-\vec{r}^{\prime}$ and $R=\|\vec{R}\|$.

In the expression (2.33), the integral involving $\underline{G}_{e}$ excludes the singularity. Due to the fact that the the field is independent from the principal volume chosen to solve the problem, the value of this Green's integral must depend on the geometry of the principal volume, since the value of $\underline{L}$ depends on it. In [90] the value of $\underline{L}$ was calculated for a spherical principal volume, whereas in [91] the value for different geometries was reported. In [92], it was presented an approach that allowed to perform numerical computations of the field by means of (2.33) using a finite principal volume $V_{\delta}$. However, in all these cases the computation of $\underline{G}_{e}$ is required and it is mainworth to compute it with high accuracy and efficiently.

Many other authors have calculated the electric dyadic Green's function in form of eigenfunction series [87-89,93]. Nevertheless, these representations of $\underline{G}_{e}$ have demonstrated to 
be inadequate for the kind of problems that we are trying to solve, since their eigenfunctions series have very poor convergence properties near the singularities even when they are avoided. In the work of Howard and Seidel [94] it was pointed out that this problem is due to the singularity associated to $\underline{G}_{e}$; they improved the convergence of the series involved by extracting a singular irrotational term (of the order $R^{-3}$ ) in closed form from the eigenfunctions series. However, the remaining series had still a singularity of the order $R^{-1}$. Also in [95] it was presented a transformation of expression (2.33) that allowed to reduce the singularity order of the dyadic Green's functions. Nevertheless, and despite this transformation, the integral expression of the field with that technique still involved a kernel diverging like $R^{-1}$.

In [82] Bressan and Conciauro overcame all these drawbacks. They extracted from the electric dyadic Green's function not only the irrotational singularity like in [94], but also a solenoidal weaker one (of the order $R^{-1}$ ). This way the remaining eigenfunction series are regular at the source point and rapidly convergent. This approach was applied to two-dimensional problems in [96] and later on used to calculate the electric dyadic Green's function of a spherical resonator in [97].

\subsubsection{Decomposition of the electric dyadic Green's function and singu- larity extraction}

The electric dyadic Green's function $\underline{G}_{e}$ for a cavity resonator involved in (2.33) is the solution of the following differential equation:

$$
\begin{aligned}
\nabla \times \nabla \times \underline{G}_{e}\left(\vec{r}, \vec{r}^{\prime}\right)-k^{2} \underline{G}_{e}\left(\vec{r}, \vec{r}^{\prime}\right) & =\underline{I} \delta\left(\vec{r}, \vec{r}^{\prime}\right) & & \text { in } V \\
\widehat{n} \times \underline{G}_{e} & =0 & & \text { on } S_{V}
\end{aligned}
$$

where $\delta\left(\vec{r}, \vec{r}^{\prime}\right)$ is the Dirac delta function in three dimensions, $\underline{I}$ is the unit dyadic, $\widehat{n}$ is the unitarty normal vector to the surface $S_{V}$ of the cavity volume $V$.

The singularity of $G_{e}$ is known to be the same as the one of the free-space Green's function $\underline{G}_{e}^{f s}$ which is given by the well-known expression:

$$
\underline{G}_{e}^{f s}\left(\vec{r}-\vec{r}^{\prime}\right)=\left(\underline{I}+k^{-2} \nabla \nabla\right) \frac{e^{-j k R}}{4 \pi R}
$$

By Taylor-expanding the exponential

$$
\frac{e^{-j k R}}{4 \pi R} \approx \frac{1}{4 \pi R}-\frac{j k}{4 \pi}-\frac{k^{2}}{8 \pi} R+\cdots
$$

and neglecting the terms finite at $R=0$, we can approximate $\underline{G}_{e}^{f s}$ at a distance much smaller 
than the wavelength $(k R \ll 1)$ by

$$
\begin{aligned}
\underline{G}_{e}^{f s}\left(\vec{r}-\vec{r}^{\prime}\right) & \approx \frac{\underline{I}}{4 \pi R}+\frac{1}{k^{2}} \nabla \nabla \frac{1}{4 \pi R}-\frac{1}{8 \pi} \nabla \nabla R \\
& \approx \frac{\underline{I}}{4 \pi R}+\frac{1}{k^{2}} \nabla \nabla \frac{1}{4 \pi R}-\frac{\underline{I}}{8 \pi R}+\frac{\vec{R} \vec{R}}{8 \pi R^{3}} \\
& \approx \frac{1}{k^{2}} \nabla \nabla \frac{1}{4 \pi R}+\frac{1}{8 \pi R}\left(\underline{I}+\frac{\vec{R} \vec{R}}{R^{2}}\right)
\end{aligned}
$$

where we have used the following identity:

$$
\begin{aligned}
\nabla \nabla R & =\nabla \sum_{i}^{3} \partial_{x_{i}} R \widehat{x}_{i}=\nabla \sum_{i}^{3} \frac{R_{i}}{R} \widehat{x}_{i}=\sum_{i j}^{3} \partial_{x_{j}} \frac{R_{i}}{R} \widehat{x}_{i} \widehat{x}_{j} \\
& =\sum_{i j}^{3} \frac{\delta_{i j} R-R_{i} R_{j} R^{-1}}{R} \widehat{x}_{i} \widehat{x}_{j}=\frac{I}{R}-\frac{\vec{R} \vec{R}}{R^{3}}
\end{aligned}
$$

The expression (2.38) reveals the nature of the singularity of $\underline{G}_{e}$ :

- There is a dominant singularity in the irrotational term $\nabla \nabla \frac{1}{4 \pi R}$, which diverges like $R^{-3}$.

- There is a weaker singularity contained in the solenoidal term $\frac{1}{8 \pi R}\left(\underline{I}+\frac{\vec{R} \vec{R}}{R^{2}}\right)$. This singularity is of the order $R^{-1}$.

The first singularity evidenced above was firstly extracted from the electric dyadic Green' function in [94], whereas the second one was extracted for the first time in the work of Bressan and Conciauro [82]. For this purpose the authors decomposed $\underline{G}_{e}$ into:

$$
\underline{G}_{e}=-\frac{1}{k^{2}} \nabla \nabla^{\prime} g^{e}\left(\vec{r}, \vec{r}^{\prime}\right)+\underline{G}_{o}^{A}\left(\vec{r}, \vec{r}^{\prime}\right)+\underline{G}_{k}^{A}\left(\vec{r}, \vec{r}^{\prime}\right)
$$

where $g^{e}\left(\vec{r}, \vec{r}^{\prime}\right)$ is the scalar Green's function; $\underline{G}_{o}^{A}\left(\vec{r}, \vec{r}^{\prime}\right)$ and $\underline{G}_{k}^{A}\left(\vec{r}, \vec{r}^{\prime}\right)$ are the solenoidal dyadics ${ }^{1}$. These functions satisfy:

$$
\begin{aligned}
\nabla^{2} g^{e}\left(\vec{r}, \vec{r}^{\prime}\right) & =-\delta\left(\vec{r}, \vec{r}^{\prime}\right) & & \text { in } \quad V \\
g^{e} & =0 & & \text { on } S_{V}
\end{aligned}
$$

and

$$
\begin{aligned}
& \nabla \times \nabla \times \underline{G}_{o}^{A}\left(\vec{r}, \vec{r}^{\prime}\right)=\underline{I} \delta\left(\vec{r}, \vec{r}^{\prime}\right)-\nabla \nabla^{\prime} g^{e}\left(\vec{r}, \vec{r}^{\prime}\right) \quad \text { in } \quad V \\
& \nabla \times \nabla \times \underline{G}_{k}^{A}\left(\vec{r}, \vec{r}^{\prime}\right)-k^{2} \underline{G}_{k}^{A}\left(\vec{r}, \vec{r}^{\prime}\right)=k^{2} \underline{G}_{o}^{A}\left(\vec{r}, \vec{r}^{\prime}\right) \quad \text { in } V \\
& \widehat{n} \times \underline{G}_{o}^{A}\left(\vec{r}, \vec{r}^{\prime}\right)=\widehat{n} \times \underline{G}_{k}^{A}\left(\vec{r}, \vec{r}^{\prime}\right)=0 \quad \text { on } S_{V}
\end{aligned}
$$

\footnotetext{
${ }^{1}$ We denote the solenoidal dyadics by $\underline{G}_{o}^{A}\left(\vec{r}, \vec{r}^{\prime}\right)$ and $\underline{G}_{k}^{A}\left(\vec{r}, \vec{r}^{\prime}\right)$ because, as we will see later in this section, they represent the vector potential dyadic Green's function $\underline{G}^{A}\left(\vec{r}, \vec{r}^{\prime}\right) . \underline{G}_{o}^{A}\left(\vec{r}, \vec{r}^{\prime}\right)$ represents a quasi-static term, independent from the frequency, and $\underline{G}_{k}^{A}\left(\vec{r}, \vec{r}^{\prime}\right)$ is the frequency dependent part.
} 
Since $\nabla \times\left(\nabla \nabla^{\prime} g^{e}\left(\vec{r}, \vec{r}^{\prime}\right)\right)=0$, using (2.41) it is easy to verify that expression (2.39) satisfies equation (2.35). On the other hand, since $\nabla \cdot \nabla \nabla^{\prime} g^{e}\left(\vec{r}, \vec{r}^{\prime}\right)=\nabla^{\prime}\left(\nabla^{2} g^{e}\right)$, using (2.40a) it can be observed that the right hand side of equation (2.41a) is solenoidal, as required for the existence of the solution of this equation.

Moreover, it is inferred from (2.40) that $g^{e} / \epsilon$ represents the electrostatic potential due to a unit point-charge placed at $\vec{r}$. It can be expressed as:

$$
g^{e}\left(\vec{r}, \vec{r}^{\prime}\right)=\frac{1}{4 \pi R}+g_{r}^{e}\left(\vec{r}, \vec{r}^{\prime}\right)
$$

where $g_{r}^{e}$ is a regular function which forces $g^{e}$ to match the boundary condition (2.40b). Hence, it satisfies:

$$
\begin{array}{ll}
\nabla^{2} g_{r}^{e}\left(\vec{r}, \vec{r}^{\prime}\right)=0 & \text { in } V \\
g_{r}^{e}=-\frac{1}{4 \pi R} & \text { on } \quad S_{V}
\end{array}
$$

Introducing now the expression (2.42) into equation (2.39), the irrotational singular term $-\frac{1}{k^{2}} \nabla \nabla^{\prime} \frac{1}{4 \pi R}$ is evidenced.

The remaining singularity, as mentioned above, is solenoidal and frequency-independent, therefore we can include it in the solenoidal dyadic $\underline{G}_{o}^{A}$. Hence, making use of the equation (2.38), we can write:

$$
\underline{G}_{o}^{A}\left(\vec{r}, \vec{r}^{\prime}\right)=\frac{1}{8 \pi R}\left(\underline{I}+\frac{\vec{R} \vec{R}}{R^{2}}\right)+\underline{G}_{o, r}^{A}\left(\vec{r}, \vec{r}^{\prime}\right)
$$

where $G_{o, r}^{A}$ is a solenoidal dyadic regular at $R=0$, and it matches the boundary conditions dictated by (2.44) together with (2.41c). As it is demonstrated in the Appendix (E), it satisfies:

$$
\nabla \times \nabla \times \underline{G}_{o, r}^{A}\left(\vec{r}, \vec{r}^{\prime}\right)=-\nabla \nabla^{\prime} g_{r}^{e}\left(\vec{r}, \vec{r}^{\prime}\right)
$$

Last, an expression for $\underline{G}_{k}^{A}$ has to be found. Since $\underline{G}_{k}^{A}$ is finite at $R=0$ and it is a solenoidal dyadic, it can be expanded in terms of the electric solenoidal eigenvectors $\vec{E}_{i}$. These eigenvectors have been demonstrated to be an orthonormal base in the previous sections, so we can write:

$$
\underline{G}_{k}^{A}\left(\vec{r}, \vec{r}^{\prime}\right)=\sum_{i}^{\infty} \vec{E}_{i}(\vec{r})\left\langle\vec{E}_{i}(\vec{r}) \cdot \underline{G}_{k}^{A}\left(\vec{r}, \vec{r}^{\prime}\right)\right\rangle
$$

Making use of the Vector-Dyadic Green's Second Theorem and the boundary conditions (2.8b) and (2.41c), it can be observed that

$$
\begin{aligned}
& \left\langle\vec{E}_{i} \cdot \nabla \times \nabla \times \underline{G}_{k}^{A}\right\rangle=\left\langle\nabla \times \nabla \times \vec{E}_{i} \cdot \underline{G}_{k}^{A}\right\rangle \\
& \left\langle\vec{E}_{i} \cdot \nabla \times \nabla \times \underline{G}_{o}^{A}\right\rangle=\left\langle\nabla \times \nabla \times \vec{E}_{i} \cdot \underline{G}_{o}^{A}\right\rangle
\end{aligned}
$$


By means of the divergence theorem and the boundary condition $(2.40 \mathrm{~b})$ and $\nabla \cdot \vec{E}_{i}=0$, it is also demonstrated that

$$
\left\langle\vec{E}_{i} \cdot \nabla \nabla^{\prime} g\left(\vec{r}, \vec{r}^{\prime}\right)\right\rangle=0
$$

Using these Green's identities together with the expressions(2.41), we can write:

$$
\begin{aligned}
\left\langle\vec{E}_{i} \cdot \underline{G}_{k}^{A}\right\rangle & =\frac{1}{k^{2}}\left\langle\vec{E}_{i} \cdot \nabla \times \nabla \times \underline{G}_{k}^{A}\right\rangle-\left\langle\vec{E}_{i} \cdot \underline{G}_{o}^{A}\right\rangle \\
& =\frac{1}{k^{2}}\left\langle\nabla \times \nabla \times \vec{E}_{i} \cdot \underline{G}_{k}^{A}\right\rangle-\left\langle\vec{E}_{i} \cdot \underline{G}_{o}^{A}\right\rangle=\frac{k_{i}^{2}}{k^{2}}\left\langle\vec{E}_{i} \cdot \underline{G}_{k}^{A}\right\rangle-\left\langle\vec{E}_{i} \cdot \underline{G}_{o}^{A}\right\rangle
\end{aligned}
$$

and with a similar procedure we find:

$$
\begin{array}{r}
\left\langle\vec{E}_{i} \cdot \underline{G}_{o}^{A}\right\rangle=\frac{1}{k_{i}^{2}}\left\langle\nabla \times \nabla \times \vec{E}_{i} \cdot \underline{G}_{o}^{A}\right\rangle=\frac{1}{k_{i}^{2}}\left\langle\vec{E}_{i} \cdot \nabla \times \nabla \times \underline{G}_{o}^{A}\right\rangle \\
=\frac{1}{k_{i}^{2}}\left(\left\langle\vec{E}_{i} \cdot \underline{I} \delta\left(\vec{r}, \vec{r}^{\prime}\right)\right\rangle-\left\langle\vec{E}_{i} \cdot \nabla \nabla^{\prime} g\left(\vec{r}, \vec{r}^{\prime}\right)\right\rangle\right)=\frac{\vec{E}_{i}}{k_{i}^{2}}
\end{array}
$$

Thus, introducing (2.47) and (2.48) in the expression (2.46), we obtain

$$
\left\langle\vec{E}_{i}(\vec{r}) \cdot \underline{G}_{k}^{A}\left(\vec{r}, \vec{r}^{\prime}\right)\right\rangle=\frac{k^{2}}{k_{i}^{2}\left(k_{i}^{2}-k^{2}\right)} \vec{E}_{i}\left(\vec{r}^{\prime}\right)
$$

Therefore, this result together with (2.42), (2.44) and (2.46) yield to the final expression of the electric dyadic Green's function

$$
\begin{aligned}
\underline{G}_{e}\left(\vec{r}, \vec{r}^{\prime}\right)= & -\frac{1}{k^{2}} \nabla \nabla^{\prime}\left[\frac{1}{4 \pi R}+g_{r}^{e}\left(\vec{r}, \vec{r}^{\prime}\right)\right]+ \\
& +\frac{1}{8 \pi R}\left(\underline{I}+\frac{\vec{R} \vec{R}}{R^{2}}\right)+\underline{G}_{o, r}^{A}\left(\vec{r}, \vec{r}^{\prime}\right)+k^{2} \sum_{i}^{\infty} \frac{\vec{E}_{i}(\vec{r}) \vec{E}_{i}\left(\vec{r}^{\prime}\right)}{k_{i}^{2}\left(k_{i}^{2}-k^{2}\right)}
\end{aligned}
$$

where both the irrotational and the solenoidal singularities are evidenced in closed form. Furthermore expressions for $g_{r}^{e}$ and $\underline{G}_{o, r}^{A}$ can be obtained in closed form or in terms of rapidly converging series depending on the geometry of the resonator cavity. The contribution of the eigenvector expansion tends to zero when $k$ tends to zero, therefore it may be seen as a 'high-frequency correction'. The most significant terms correspond to resonances occurring not much above the operating frequency, since the terms of the series decrease as $k_{i}^{-4}$ when $k_{i}^{2}$ becomes much larger than $k^{2}$.

\subsubsection{Physical meaning of the electric dyadic Green's function decom- position: The Coulomb Gauge.}

So far we have found an expression for the electric dyadic Green's function that allows us to extract the singularities of $\underline{G}_{e}$ and to calculate it in an accurate and efficient way. We can 
now give a physical interpretation to this expression. By substituting (2.39) into (2.33) and making use of (2.50), we can write:

$$
\vec{E}(\vec{r})=\vec{E}_{o}(\vec{r})+\vec{E}_{A}(\vec{r})+\widetilde{\vec{E}}(\vec{r})
$$

where

$$
\begin{aligned}
\vec{E}_{o}(\vec{r}) & =j \frac{j \eta}{k}\left[\lim _{\delta \rightarrow 0} \int_{V-V_{\delta}}\left(\nabla \nabla^{\prime} g^{e}\left(\vec{r}, \vec{r}^{\prime}\right)\right) \cdot \vec{J}\left(\vec{r}^{\prime}\right) d v^{\prime}+\underline{L} \cdot \vec{J}(\vec{r})\right] \\
\vec{E}_{A}(\vec{r}) & =-j k \eta \int_{V} \underline{G}_{o}^{A}\left(\vec{r}, \vec{r}^{\prime}\right) \cdot \vec{J}\left(\vec{r}^{\prime}\right) d v^{\prime} \\
\widetilde{\vec{E}}(\vec{r}) & =-j k^{3} \eta \sum_{i}^{\infty} \frac{1}{k_{i}^{2}\left(k_{i}^{2}-k^{2}\right)} \vec{E}_{i}(\vec{r}) \int_{V} \vec{E}_{i}\left(\vec{r}^{\prime}\right) \cdot \vec{J}\left(\vec{r}^{\prime}\right) d v^{\prime}
\end{aligned}
$$

In the expression (2.52b), the limit has been omitted because the singularity of $\underline{G}_{o}^{A}$ is of the order of $R^{-1}$ and hence it is integrable [98], being the integral independent from the choice of the principal volume. The expression of $\vec{E}_{o}$ can be further developed using the divergence theorem, thus obtaining

$$
\begin{aligned}
& \lim _{\delta \rightarrow 0} \int_{V-V_{\delta}}\left[\nabla \nabla^{\prime} g^{e}\left(\vec{r}, \vec{r}^{\prime}\right)\right] \cdot \vec{J}\left(\vec{r}^{\prime}\right) d v^{\prime}= \\
& =\lim _{\delta \rightarrow 0} \int_{V-V_{\delta}} \nabla\left[\nabla^{\prime} \cdot\left(g^{e}\left(\vec{r}, \vec{r}^{\prime}\right) \vec{J}\left(\vec{r}^{\prime}\right)\right)-g^{e}\left(\vec{r}, \vec{r}^{\prime}\right) \nabla^{\prime} \cdot \vec{J}\left(\vec{r}^{\prime}\right)\right] d v^{\prime}= \\
& =\int_{S_{V}} \nabla g^{e}\left(\vec{r}, \vec{r}^{\prime}\right) \vec{J}\left(\vec{r}^{\prime}\right) \cdot \widehat{n} d s^{\prime}+\lim _{\delta \rightarrow 0} \int_{s_{\delta}} \nabla g^{e}\left(\vec{r}, \vec{r}^{\prime}\right) \widehat{n} \cdot \vec{J}\left(\vec{r}^{\prime}\right) d s^{\prime}- \\
& -\int_{V} \nabla g^{e}\left(\vec{r}, \vec{r}^{\prime}\right) \nabla^{\prime} \cdot \vec{J}\left(\vec{r}^{\prime}\right) d v^{\prime}
\end{aligned}
$$

Since $g^{e}\left(\vec{r}, \vec{r}^{\prime}\right)$ vanishes for any value of $\vec{r}^{\prime}$ on the surface of the resonator cavity, then the first surface integral in (2.53) is equal to zero. The volume integral, once again, does not depend on the principal volume because the singularity of $\nabla g^{e}$ is of the order of $R^{-2}$ and it is integrable [98], thus we have omitted the reference to the principal value. Using (2.42) we can write:

$$
\begin{aligned}
& \lim _{\delta \rightarrow 0} \int_{s_{\delta}} \nabla g^{e}\left(\vec{r}, \vec{r}^{\prime}\right) \widehat{n} \cdot \vec{J}\left(\vec{r}^{\prime}\right) d s^{\prime}= \\
& =\lim _{\delta \rightarrow 0} \int_{s_{\delta}} \nabla\left(\frac{1}{4 \pi R}\right) \widehat{n} \cdot \vec{J}\left(\vec{r}^{\prime}\right) d s^{\prime}+\int_{s_{\delta}} \nabla g_{r}^{e}\left(\vec{r}, \vec{r}^{\prime}\right) \widehat{n} \cdot \vec{J}\left(\vec{r}^{\prime}\right) d s^{\prime}= \\
& =-\lim _{\delta \rightarrow 0} \int_{s_{\delta}}\left(\frac{\vec{R}}{4 \pi R^{-3}}\right) \widehat{n} \cdot \vec{J}\left(\vec{r}^{\prime}\right) d s^{\prime}=-\underline{L} \cdot \vec{J}(\vec{r})
\end{aligned}
$$

where we have taken into consideration that $\vec{J}(\vec{r})$ and $g_{r}^{e}\left(\vec{r}, \vec{r}^{\prime}\right)$ are regular functions at $\vec{r}=\vec{r}^{\prime}$, and so the integral containing $g_{r}^{e}\left(\vec{r}, \vec{r}^{\prime}\right)$ vanishes in the limit. Moreover, in the last integral, we can assume $\vec{J}\left(\vec{r}^{\prime}\right)$ to have a fixed value $\left(\vec{J}\left(\vec{r}^{\prime}\right)=\vec{J}(\vec{r})\right)$ when $\delta$ tends to zero. 
Substituting (2.54) in (2.53) and introducing it in (2.52a), we obtain

$$
\vec{E}_{o}(\vec{r})=-\frac{1}{\epsilon} \nabla \int_{V} g^{e}\left(\vec{r}, \vec{r}^{\prime}\right) \rho\left(\vec{r}^{\prime}\right) d v^{\prime}
$$

where we have used the continuity equation $\rho(\vec{r})=-\nabla \cdot \vec{J}(\vec{r}) / j \omega$.

It is remarkable that it has been obtained a expression for the electric field which is independent from the principal volume chosen. Furthermore, equation (2.55) permits to recognize $\vec{E}_{o}$ as the electric field given by the gradient of the scalar potential in the Coulomb gauge. As a consequence the solenoidal terms $\vec{E}_{A}(\vec{r})+\widetilde{\vec{E}}(\vec{r})$ represent the contribution given by the potential vector in the same gauge.

\subsection{Scalar and vector potentials (Coulomb gauge)}

In the previous section we have presented an expression of the electric dyadic Green's function that permits to calculate efficiently the field in a cavity resonator. It is a decomposition of $\underline{G}_{e}$ in which the singularities are evidenced in closed form and the remaining series are rapidly converging. Moreover the integrals involved does not depend on the principal volume chosen to calculate them like it occurs when using directly the equation $(2.33)[90,91]$. As we have seen this decomposition yields to a representation of the field in the Coulomb gauge. Moreover, in problems where the sources depend on the field (we will see that this is our case in the next chapter), it is crucial to calculate with high accuracy and efficiency the electric dyadic Green's function. Due to all these reasons, we will choose the Coulomb gauge to represent the electromagnetic field.

Furthermore, in equations (2.20) we have split the field into solenoidal and irrotational series expansion. The same kind of decomposition is present in the expression of the field by means of the scalar and vector potentials in the Coulomb gauge $[49,99]$ :

$$
\begin{aligned}
\vec{E} & =-\nabla V^{e}-j \omega \vec{A}-\frac{1}{\epsilon} \nabla \times \vec{F} \\
\vec{H} & =-\nabla V^{m}+\frac{1}{\mu} \nabla \times \vec{A}-j \omega \vec{F}
\end{aligned}
$$

where $V^{e}$ is the electric scalar potential, $V^{m}$ is the magnetic scalar potential, $\vec{A}$ denotes the electric vector potential, and $\vec{F}$ denotes the magnetic vector potential.

In the Coulomb gauge, both the electric and magnetic vector potentials are solenoidal,

$$
\nabla \cdot \vec{A}=\nabla \cdot \vec{F}=0
$$

The electric potential vector satisfies:

$$
\begin{aligned}
\nabla \times \nabla \times \vec{A}-k^{2} \vec{A} & =\mu \vec{J}-j \omega \mu \epsilon \nabla V^{e} & & \text { in } \quad V \\
\widehat{n} \times \vec{A} & =0 & & \text { on } S_{V}
\end{aligned}
$$


whereas in the case of the magnetic vector potential, the equations are:

$$
\begin{aligned}
& \nabla \times \nabla \times \vec{F}-k^{2} \vec{F}=\epsilon \vec{M}-j \omega \mu \epsilon \nabla V^{m} \quad \text { in } \quad V \\
& \widehat{n} \times \nabla \times \vec{F}=0 \quad \text { on } S_{V}
\end{aligned}
$$

The static electric potential is irrotational and satisfies:

$$
\begin{aligned}
& \nabla^{2} V^{e}=-\frac{\rho^{e}}{\epsilon} \quad \text { in } \quad V \\
& V^{e}=0 \quad \text { on } S_{V}
\end{aligned}
$$

where $\rho^{e}$ is the electric charge density which verifies the continuity equation $\nabla \cdot \vec{J}=-j \omega \rho^{e}$.

The static magnetic potential is also irrotational and it satisfies:

$$
\begin{aligned}
\nabla^{2} V^{m} & =-\frac{\rho^{m}}{\mu} & & \text { in } & V \\
\frac{\partial V^{e}}{\partial n} & =0 & & \text { on } & S_{V}
\end{aligned}
$$

where $\rho^{m}$ is the magnetic charge density which verifies the continuity equation $\nabla \cdot \vec{M}=$ $-j \omega \rho^{m}$.

We may express now the scalar and vector potentials as an eigenvector expansion proceeding like in section 2.3 to solve the equations above. Nevertheless, it is simpler to compare equations (2.56) with (2.32), and taking into account which terms are solenoidal or irrotational and their dependency on $\vec{J}$ or $\vec{M}$, we can obtain the following representation of the electric scalar and vector potential:

$$
\begin{gathered}
\vec{A}=\mu \sum_{i=1}^{\infty} \frac{\left\langle\vec{E}_{i}, \vec{J}\right\rangle}{k_{i}^{2}-k^{2}} \vec{E}_{i} \\
\nabla \times \vec{A}=\mu \sum_{i=1}^{\infty} \frac{k_{i}\left\langle\vec{E}_{i}, \vec{J}\right\rangle}{k_{i}^{2}-k^{2}} \vec{H}_{i} \\
\nabla V^{e}=-j \frac{\eta}{k} \sum_{i=1}^{\infty}\left\langle\vec{f}_{i}, \vec{J}\right\rangle \vec{f}_{i}
\end{gathered}
$$

Introducing now (2.5) in (2.64), we can write:

$$
\nabla V^{e}(\vec{r})=-j \frac{\eta}{k} \sum_{i=1}^{\infty}\left\langle\vec{f}_{i}, \vec{J}\right\rangle \frac{\nabla \phi_{i}(\vec{r})}{\mu_{i}}
$$

and assuming that differentiation and summation can be inverted ${ }^{2}$, we obtain

$$
V^{e}(\vec{r})=-j \frac{\eta}{k} \sum_{i=1}^{\infty}\left\langle\vec{f}_{i}, \vec{J}\right\rangle \frac{\phi_{i}}{\mu_{i}}
$$


Moreover, we have:

$$
\left\langle\vec{f}_{i}, \vec{J}\right\rangle=\frac{1}{\mu_{i}} \int_{V} \nabla \phi_{i} \cdot \vec{J} d v=-\frac{1}{\mu_{i}} \int_{V} \phi_{i} \nabla \cdot \vec{J} d v=\frac{j \omega}{\mu_{i}} \int_{V} \phi_{i} \rho^{e} d v
$$

where we have used the divergence theorem, the continuity equation and that $\phi_{i}=0$ on the boundary. Thus we can finally rewrite (2.66) as

$$
V^{e}(\vec{r})=\frac{1}{\epsilon} \sum_{i=1}^{\infty} \frac{\phi_{i}(\vec{r})}{\mu_{i}^{2}} \int_{V} \phi_{i} \rho^{e} d v
$$

The expansion for the magnetic scalar and vector potentials can be analogously obtained:

$$
\begin{gathered}
\vec{F}=\epsilon \sum_{i=1}^{\infty} \frac{\left\langle\vec{H}_{i}, \vec{M}\right\rangle}{k_{i}^{2}-k^{2}} \vec{H}_{i} \\
\nabla \times \vec{F}=\epsilon \sum_{i=1}^{\infty} k_{i} \frac{\left\langle\vec{H}_{i}, \vec{M}\right\rangle}{k^{2}-k_{i}^{2}} \vec{E}_{i} \\
\nabla V^{m}=\frac{1}{j \eta k} \sum_{i=1}^{\infty}\left\langle\vec{g}_{i}, \vec{M}\right\rangle \vec{g}_{i}
\end{gathered}
$$

To obtain the expansion of $V^{m}$, we have to consider the following expression:

$$
\left\langle\vec{g}_{i}, \vec{M}\right\rangle=\frac{1}{\nu_{i}} \int_{V} \nabla \psi_{i} \cdot \vec{J} d v=-\frac{1}{\nu_{i}} \int_{V} \psi_{i} \nabla \cdot \vec{J} d v=\frac{j \omega}{\nu_{i}} \int_{V} \psi_{i} \rho^{m} d v
$$

where we have made use of the equation (2.13), the divergence theorem, the continuity equation for the magnetic sources and the boundary condition $\widehat{n} \cdot \vec{M}=0$. Thus, proceeding like for the electric scalar potential, we have ${ }^{2}$

$$
V^{m}(\vec{r})=\frac{1}{\mu} \sum_{i=1}^{\infty} \frac{\psi_{i}(\vec{r})}{\nu_{i}^{2}} \int_{V} \psi_{i} \rho^{m} d v
$$

\footnotetext{
${ }^{2}$ The eigenvectors used in the expansion of the scalar and the vector potentials satisfy the same boundary conditions as the potentials. This fact makes the series to be uniform convergent [85], and thus it is possible to invert the differentiation or integration and the summation in the expressions (2.62), (2.67), (2.68), and (2.71). In fact, it is possible to obtain equations (2.63) and (2.69) by differentiating termwise (2.62) and (2.68) respectively.
} 


\subsection{Potential Green's functions}

In the preceding section we have expressed the scalar and vector potentials for a cavity resonator with perfect conducting walls as an eigenvector expansion (see equations (2.62), (2.67), (2.68), and (2.71)). By inverting now the integration and the summation ${ }^{2}$, we obtain the representation of the potentials in an integral form:

$$
\begin{gathered}
V^{e}=\frac{1}{\epsilon} \int_{V} g^{e}\left(\vec{r}, \vec{r}^{\prime}\right) \rho^{e}\left(\vec{r}^{\prime}\right) d v^{\prime} \\
V^{m}=\frac{1}{\epsilon} \int_{V} g^{m}\left(\vec{r}, \vec{r}^{\prime}\right) \rho^{m}\left(\vec{r}^{\prime}\right) d v^{\prime} \\
\vec{A}=\mu \int_{V} \underline{G}^{A}\left(\vec{r}, \vec{r}^{\prime}\right) \cdot \vec{J}\left(\vec{r}^{\prime}\right) d v^{\prime} \\
\vec{F}=\mu \int_{V} \underline{G}^{F}\left(\vec{r}, \vec{r}^{\prime}\right) \cdot \vec{M}\left(\vec{r}^{\prime}\right) d v^{\prime}
\end{gathered}
$$

where we have defined:

$$
\begin{aligned}
g^{e}\left(\vec{r}, \vec{r}^{\prime}\right) & =\sum_{i}^{\infty} \frac{\phi_{i}(\vec{r}) \phi_{i}\left(\vec{r}^{\prime}\right)}{\mu_{i}^{2}} \\
g^{m}\left(\vec{r}, \vec{r}^{\prime}\right) & =\sum_{i}^{\infty} \frac{\psi_{i}(\vec{r}) \psi_{i}\left(\vec{r}^{\prime}\right)}{\nu_{i}^{2}} \\
\underline{G}^{A}\left(\vec{r}, \vec{r}^{\prime}\right) & =\sum_{i}^{\infty} \frac{\vec{E}_{i}(\vec{r}) \vec{E}_{i}\left(\vec{r}^{\prime}\right)}{k_{i}^{2}-k^{2}} \\
\underline{G}^{F}\left(\vec{r}, \vec{r}^{\prime}\right) & =\sum_{i}^{\infty} \frac{\vec{H}_{i}(\vec{r}) \vec{H}_{i}\left(\vec{r}^{\prime}\right)}{k_{i}^{2}-k^{2}}
\end{aligned}
$$

where $g^{e}$ and $g^{m}$ are the scalar Green's functions for the electric and magnetic scalar potential, whereas $\underline{G}^{A}$ and $\underline{G}^{F}$ are the dyadic Green's functions for the electric and magnetic vector potentials, respectively.

The $g^{e}$ function coincides with the Green's function for the electrostatic potential in the cavity according to the Coulomb's gauge and, as it has been presented in section 2.4 , it satisfies:

$$
\begin{aligned}
\nabla^{2} g^{e}\left(\vec{r}, \vec{r}^{\prime}\right) & =-\delta\left(\vec{r}, \vec{r}^{\prime}\right) & & \text { in } \quad V \\
g^{e} & =0 & & \text { on } S_{V}
\end{aligned}
$$


The equations satisfied by the magnetic Green's function are dual:

$$
\begin{aligned}
\nabla^{2} g^{m}\left(\vec{r}, \vec{r}^{\prime}\right) & =-\delta\left(\vec{r}, \vec{r}^{\prime}\right) & & \text { in } \quad V \\
\frac{\partial g^{m}}{\partial n} & =0 & & \text { on } \quad S_{V}
\end{aligned}
$$

To obtain the differential equation satisfied by the dyadic $\underline{G}^{A}$, we perform the following substitutions in eq.(2.58):

$$
\mu \vec{J} \Rightarrow \underline{I} \delta\left(\vec{r}, \vec{r}^{\prime}\right) \quad \vec{A}(\vec{r}) \Rightarrow \underline{G}^{A}\left(\vec{r}, \vec{r}^{\prime}\right)
$$

and

$$
\mu\left(j \omega \epsilon \nabla V^{e}\right) \Rightarrow \nabla \int_{V} \nabla^{\prime \prime} g^{e}\left(\vec{r}, \vec{r}^{\prime \prime}\right) \cdot \underline{I} \delta\left(\vec{r}^{\prime \prime}-\vec{r}^{\prime}\right) d v^{\prime \prime}=\nabla \nabla^{\prime} g^{e}\left(\vec{r}, \vec{r}^{\prime}\right)
$$

The last transformation has been proposed because

$$
\begin{aligned}
\mu\left(j \omega \epsilon \nabla V^{e}\right) & =j \omega \mu \nabla \int_{V} g^{e}\left(\vec{r}, \vec{r}^{\prime \prime}\right) \rho^{e}\left(\vec{r}^{\prime \prime}\right) d v^{\prime \prime}= \\
& =-\mu \nabla \int_{V} g^{e}\left(\vec{r}, \vec{r}^{\prime \prime}\right) \nabla^{\prime \prime} \vec{J}\left(\vec{r}^{\prime \prime}\right) d v^{\prime \prime}=\nabla \int_{V} \nabla^{\prime \prime} g^{e}\left(\vec{r}, \vec{r}^{\prime \prime}\right) \mu \vec{J}\left(\vec{r}^{\prime \prime}\right) d v^{\prime \prime}
\end{aligned}
$$

where we have used the divergence theorem and the condition $g^{e} \vec{J}=0$ on the boundary. In conclusion, $\underline{G}^{A}$ is the solution of the differential equation:

$$
\begin{aligned}
\nabla \times \nabla \times \underline{G}^{A}\left(\vec{r}, \vec{r}^{\prime}\right)-k^{2} \underline{G}^{A}\left(\vec{r}, \vec{r}^{\prime}\right) & =\underline{I} \delta\left(\vec{r}, \vec{r}^{\prime}\right)-\nabla \nabla^{\prime} g^{e}\left(\vec{r}, \vec{r}^{\prime}\right) & \text { in } \quad V \\
\widehat{n} \times \underline{G}^{A}\left(\vec{r}, \vec{r}^{\prime}\right) & =0 & \text { on } \quad S_{V}
\end{aligned}
$$

As mentioned in previous sections, the series in expressions (2.73) converge very slowly, due to the singular behaviour of the Green's functions when $\vec{r}$ and $\vec{r}^{\prime}$ coalesce. When performing numerical calculations, these series need to be truncated and the slow convergence may yield to inaccurate results in the source region. It has been shown that this drawback can be avoided by extracting the Green's functions singularities in closed form.

Since the form of the singularity does not depend on the boundary conditions, it is the same for both the electric and magnetic scalar Green's function:

$$
g_{s}\left(\vec{r}, \vec{r}^{\prime}\right)=\frac{1}{4 \pi R}
$$

and thus, we may decompose the scalar Green's function like in section (2.4):

$$
g^{e}\left(\vec{r}, \vec{r}^{\prime}\right)=g_{s}\left(\vec{r}, \vec{r}^{\prime}\right)+g_{r}^{e}\left(\vec{r}, \vec{r}^{\prime}\right) \quad g^{m}\left(\vec{r}, \vec{r}^{\prime}\right)=g_{s}\left(\vec{r}, \vec{r}^{\prime}\right)+g_{r}^{m}\left(\vec{r}, \vec{r}^{\prime}\right)
$$


where $g_{r}^{e}$ and $g_{r}^{m}$ are regular functions at $R=0$.

In the case of $\underline{G}^{A}$, since the singularity is independent from the frequency, we have shown that the series expansion can be accelerated by extracting its zero-frequency limit $G_{o}^{A}$. Thus, we can write:

$$
\underline{G}^{A}\left(\vec{r}, \vec{r}^{\prime}\right)=\underline{G}_{o}^{A}\left(\vec{r}, \vec{r}^{\prime}\right)+\underline{G}_{k}^{A}\left(\vec{r}, \vec{r}^{\prime}\right) \quad \text { with } \quad \underline{G}_{o}^{A}\left(\vec{r}, \vec{r}^{\prime}\right)=\left.\underline{G}^{A}\left(\vec{r}, \vec{r}^{\prime}\right)\right|_{k=0}
$$

Introducing eq.(2.80) in (2.73c), we can easily verify that

$$
\underline{G}_{k}^{A}\left(\vec{r}, \vec{r}^{\prime}\right)=k^{2} \sum_{i}^{\infty} \frac{\vec{E}_{i}(\vec{r}) \vec{E}_{i}\left(\vec{r}^{\prime}\right)}{k_{i}^{2}\left(k_{i}^{2}-k^{2}\right)}
$$

This is the expression obtained using (2.49) and (2.46). In fact, by introducing the equations (2.80), (2.81) in (2.77), it is easily demonstrated that equations (2.41) are satisfied.

In conclusion, using the results obtained in section 2.4, we can write:

$$
\begin{aligned}
\underline{G}^{A}\left(\vec{r}, \vec{r}^{\prime}\right) & =\underline{G}_{o}^{A}\left(\vec{r}, \vec{r}^{\prime}\right)+\underline{G}_{k}^{A}\left(\vec{r}, \vec{r}^{\prime}\right)= \\
& =\frac{1}{8 \pi R}\left(\underline{I}+\frac{\vec{R} \vec{R}}{R^{2}}\right)+\underline{G}_{o, r}^{A}\left(\vec{r}, \vec{r}^{\prime}\right)+k^{2} \sum_{i}^{\infty} \frac{\vec{E}_{i}(\vec{r}) \vec{E}_{i}\left(\vec{r}^{\prime}\right)}{k_{i}^{2}\left(k_{i}^{2}-k^{2}\right)}
\end{aligned}
$$

The dual expression gives us the dyadic Green's function for the electric vector potential:

$$
\begin{aligned}
\underline{G}^{F}\left(\vec{r}, \vec{r}^{\prime}\right) & =\underline{G}_{o}^{F}\left(\vec{r}, \vec{r}^{\prime}\right)+\underline{G}_{k}^{F}\left(\vec{r}, \vec{r}^{\prime}\right)= \\
& =\frac{1}{8 \pi R}\left(\underline{I}+\frac{\vec{R} \vec{R}}{R^{2}}\right)+\underline{G}_{o, r}^{F}\left(\vec{r}, \vec{r}^{\prime}\right)+k^{2} \sum_{i}^{\infty} \frac{\vec{H}_{i}(\vec{r}) \vec{H}_{i}\left(\vec{r}^{\prime}\right)}{k_{i}^{2}\left(k_{i}^{2}-k^{2}\right)}
\end{aligned}
$$

where $\underline{G}_{o, r}^{A}$ and $\underline{G}_{o, r}^{F}$ are regular dyadic functions at $R=0$.

So finally, we have extracted the singularities from the Green's functions in such a way that we are able now to express them in terms of rapidly converging series. Expressions for $g_{r}^{e}, g_{r}^{m}, G_{o, r}^{A}$ and $\underline{G}_{o, r}^{F}$ can be found using different methods, such as the image technique. For instance, in the case of a spherical resonator, expressions in closed forms can be found $[62,63,73,97]$. In the case of the present work, we will use the Green's functions of a rectangular cavity which can be expressed as a triple series form. The computation of these series can be tedious and different techniques to accelerate it have been proposed [100]. Nevertheless, in this work we will use a method presented in [101] based on the Ewald technique [102]. This method has been applied in different works, such as [74, 75, 101], demonstrating to be very efficient. Its implementation has been reported in detail in [Ch. 3, $103]$ and in [104]. Moreover, in order to compute the dyadic Green's functions, the infinite series in (2.82) and (2.83) must be truncated, so we finally express the dyadic Green fucntion as:

$$
\begin{array}{r}
\underline{G}^{A}\left(\vec{r}, \vec{r}^{\prime}\right)=\frac{1}{8 \pi R}\left(\underline{I}+\frac{\vec{R} \vec{R}}{R^{2}}\right)+\underline{G}_{o, r}^{A}\left(\vec{r}, \vec{r}^{\prime}\right)+k^{2} \sum_{i}^{M} \frac{\vec{E}_{i}(\vec{r}) \vec{E}_{i}\left(\vec{r}^{\prime}\right)}{k_{i}^{2}\left(k_{i}^{2}-k^{2}\right)} \\
\left(k<k_{\max } ; \quad k_{M} \leq \xi k_{\max }<k_{M+1}\right)
\end{array}
$$


where $k_{\max }$ is the wavenumber correspondent to the highest frequency of interest, and $\xi$ is a numerical parameter greater than 1 . This parameter can be regarded as an accuracy factor, since the number of modes considered in the series grows up as $\xi$ increases. Its value is typically around $\xi \approx 2,3[62,73]$. Obviously, the dual expression of (2.84) holds to compute the dyadic Green's function for the magnetic vector potential.

\subsection{Hybrid representation of the electromagnetic field in an ideal cavity}

In order to construct now the expressions of the fields, we first introduce equations (2.72a) and (2.72c) in (2.56), making use of (2.76) and inverting the curl and the integration ${ }^{3}$ in $(2.72 \mathrm{~d})$,

$$
\nabla \times \vec{F}=\epsilon \int_{V} \nabla \times \underline{G}^{F}\left(\vec{r}, \vec{r}^{\prime}\right) \cdot \vec{M}\left(\vec{r}^{\prime}\right) d v^{\prime}
$$

we can obtain the expression of the electric field in terms of the Green's functions:

$$
\begin{aligned}
\vec{E}(\vec{r})= & \frac{\eta}{j k} \nabla \int_{V} g^{e}\left(\vec{r}, \vec{r}^{\prime}\right) \nabla^{\prime} \cdot \vec{J}\left(\vec{r}^{\prime}\right) d v^{\prime}-j k \eta \int_{V} \underline{G}^{A}\left(\vec{r}, \vec{r}^{\prime}\right) \cdot \vec{J}\left(\vec{r}^{\prime}\right) d v^{\prime} \\
& -\int_{V} \nabla \times \underline{G}^{F}\left(\vec{r}, \vec{r}^{\prime}\right) \cdot \vec{M}\left(\vec{r}^{\prime}\right) d v^{\prime}
\end{aligned}
$$

And analogously, the expression for the magnetic field can be found:

$$
\begin{aligned}
\vec{H}(\vec{r})= & \frac{1}{j k \eta} \nabla \int_{V} g^{m}\left(\vec{r}, \vec{r}^{\prime}\right) \nabla^{\prime} \cdot \vec{M}\left(\vec{r}^{\prime}\right) d v^{\prime}-\frac{j k}{\eta} \int_{V} \underline{G}^{F}\left(\vec{r}, \vec{r}^{\prime}\right) \cdot \vec{M}\left(\vec{r}^{\prime}\right) d v^{\prime} \\
& +\int_{V} \nabla \times \underline{G}^{A}\left(\vec{r}, \vec{r}^{\prime}\right) \cdot \vec{J}\left(\vec{r}^{\prime}\right) d v^{\prime}
\end{aligned}
$$

And finally, by introducing equations (2.82) and (2.83) in these expressions, we obtain the 'hybrid' representation of the field:

$$
\begin{aligned}
\vec{E}(\vec{r}) & =\frac{\eta}{j k} \nabla \int_{V} g^{e}\left(\vec{r}, \vec{r}^{\prime}\right) \nabla^{\prime} \cdot \vec{J}\left(\vec{r}^{\prime}\right) d v^{\prime} \\
& -j k \eta \int_{V} \underline{G}_{o}^{A}\left(\vec{r}, \vec{r}^{\prime}\right) \cdot \vec{J}\left(\vec{r}^{\prime}\right) d v^{\prime}-\int_{V} \nabla \times \underline{G}_{o}^{F}\left(\vec{r}, \vec{r}^{\prime}\right) \cdot \vec{M}\left(\vec{r}^{\prime}\right) d v^{\prime} \\
& -j k^{3} \sum_{i}^{\infty} \frac{\int_{V} \vec{E}_{i}\left(\vec{r}^{\prime}\right) \cdot \vec{J}\left(\vec{r}^{\prime}\right) d v^{\prime}}{k_{i}^{2}\left(k_{i}^{2}-k^{2}\right)} \vec{E}_{i}(\vec{r})-k^{2} \sum_{i}^{\infty} \frac{\int_{V} \vec{H}_{i}\left(\vec{r}^{\prime}\right) \cdot \vec{M}\left(\vec{r}^{\prime}\right) d v^{\prime}}{k_{i}^{2}\left(k_{i}^{2}-k^{2}\right)} \vec{H}_{i}(\vec{r})
\end{aligned}
$$

\footnotetext{
${ }^{3}$ We can invert the differential operator and the integration because the singularity of $\underline{G}^{F}$ is integrable (of the order $R^{-1}$ ), and it does not depend on the principal volume. If we were working with $\underline{G}_{e}$, which as shown has a higher singularity $\left(R^{-3}\right)$, this interchange of operators will give problems (the well-known problem of the classical delta-function method [91]).
} 


$$
\begin{aligned}
\vec{H}(\vec{r}) & =\frac{1}{j k \eta} \nabla \int_{V} g^{m}\left(\vec{r}, \vec{r}^{\prime}\right) \nabla^{\prime} \cdot \vec{M}\left(\vec{r}^{\prime}\right) d v^{\prime} \\
& -\frac{j k}{\eta} \int_{V} \underline{G}_{o}^{F}\left(\vec{r}, \vec{r}^{\prime}\right) \cdot \vec{M}\left(\vec{r}^{\prime}\right) d v^{\prime}+\int_{V} \nabla \times \underline{G}_{o}^{A}\left(\vec{r}, \vec{r}^{\prime}\right) \cdot \vec{J}\left(\vec{r}^{\prime}\right) d v^{\prime} \\
& -\frac{j k^{3}}{\eta} \sum_{i}^{\infty} \frac{\int_{V} \vec{H}_{i}\left(\vec{r}^{\prime}\right) \cdot \vec{M}\left(\vec{r}^{\prime}\right) d v^{\prime}}{k_{i}^{2}\left(k_{i}^{2}-k^{2}\right)} \vec{H}_{i}(\vec{r})+k^{2} \sum_{i}^{\infty} \frac{\int_{V} \vec{E}_{i}\left(\vec{r}^{\prime}\right) \cdot \vec{J}\left(\vec{r}^{\prime}\right) d v^{\prime}}{k_{i}^{2}\left(k_{i}^{2}-k^{2}\right)} \vec{E}_{i}(\vec{r})
\end{aligned}
$$

The fields are given partly by eigenvector expansions and partly by Green's integrals. The Green integrals represent the field at low frequency. In order to compute them accurately, it is crucial extract the Green's function singularities, and to properly transform these singular integrals. The eigenvector series represent high-frequency corrections and they converge much faster than the full eigenvector expansions of the electric and magnetic fields. 


\section{Chapter 3}

\section{State-Space Integral-Equation Formulation based on the BI-RME Method}

\subsection{Introduction}

During the last decades, a great number of numerical techniques have been developed to characterise the electromagnetic response of passive microwave circuits by means of frequencyand time-domain procedures $[47,59]$. In time-domain methods, such as the finite-difference time-domain (FDTD) or the transmission-line matrix (TLM) algorithm, the temporal evolution of the field (time-step by time-step) is computed by discretizing the Maxwell's equations both in time and space. In a frequency-domain method, such as the finite-element method (FEM) and boundary element methods (integral equation techniques or mode matching method), the Maxwell's equations are written in the frequency domain and solved to obtain the field in the solution region computed at a set frequency. In any case, by making use of this kind of procedures, problems must be solved over and over again to find the frequency response in a broad bandwith. This drawback can be avoided using $s$-domain procedures ( $s$ is the Laplace variable).

From the 90's up to nowadays, several studies have been devoted to the development of new electromagnetic solvers for the analysis of passive microwave devices through state space-domain methods $[60,61]$. These mathematical models permit to represent a linear system described by partial differential equations in terms of an infinite poles and zeros expansion. In other words, one can obtain the electromagnetic response of a microwave device in terms of some circuit generalized matrix (scattering, admittance, impedance, etc.) represented in the form of pole expansion in the Laplace variable $s$. $S$-domain methods present several advantages: since the system is linear, there is no need to compute over and over again by stepping through time or frequency, and thus, frequency sweeps are computed very fast. Furthermore, $s$-domain solutions may be cast into equivalent electrical circuits, 
which is crucial in synthesis and design processes.

In the case of distributed circuits, finite difference (FD) and finite element (FE) methods applied to the Maxwell equations in the $s$-domain yield to equations in which the discretized field is represented by the state variables. However, these methods need to perform refined 3D meshes, and consequently they make use of huge computational resources, slowing down significantly the numerical computations performed, specially when complex 3D structures are analysed. On the other hand, boundary element methods (BEM) avoid 3D meshes, but do not give rise directly to mathematical models in the form of pole expansions valid on a very wide bands, though fast frequency sweeps can be achieved by the adaptive Lanczos-Padè algorithm [60,61].

In this work we obtain the generalized admittance matrix (GAM) of a dielectric-loaded cavity resonator in terms of a pole expansion in the Laplace variable domain by means of a state-space integral-equation (SS-IE) approach. This technique is based in the well-known 'Boundary -Integral Resonant Mode Expansion' (BI-RME) method developed in the University of Pavia (Italy).

The BI-RME method was formerly applied to obtain the normalized resonant modes of arbitrarily shaped metallic cavities in [63]. The unknown current flowing on the cavity walls was considered inside a spherical resonator, rather than in free space as it is used to be done in boundary element methods. Thus, by using the Green's functions of the spherical resonator instead of the free space one, the problem was cast by the Method of Moments (MoM) into a real matrix linear eigenvalue problem. Consequently, this technique avoids frequency-perfrequency computations, resulting in a very efficient tool. The same approach had been used some years before to obtain the modes of arbitrarily shaped metallic waveguides [64], which was further enhanced in [65].

Making use of the Kurokawa's representation of the field in a cavity resonator (see chapter 2), it is possible to relate the pole expansion of the admittance parameters to the resonant modes of the cavity obtained by closing the structure ports with conducting planes. Following this procedure, a very efficient algorithm was developed in $[62,66-68]$ to obtain the pole expansion of the admittance matrix based on the BI-RME method and the use of very simple expressions to represent the low-frequency behaviour of the Y-matrix. However, the low-frequency approximation introduces a limitation in the algorithm, since the ports of the structure have to be long enough to separate the waveguides connected to the discontinuities. In subsequent years, new procedures were developed in order to overcome this drawback. In [69-71], the generalized admittance matrix of 2D and 3D structures was obtained by using the BI-RME method to obtain the resonant modes and a different technique to find the quasi-static approximation of the GAM. It is remarkable that the proper combination of the generalized admittance matrices that characterize different building blocks in which a whole circuit is subdivided can yield to a GAM in the form of a pole expansion that characterize the whole structure [72].

It was in [73] where a unified algorithm based only in the BI-RME method was definitively presented. In that work, the pole expansion of the GAM in the $s$-domain was directly derived from the BI-RME method. Furthermore, since the meshing procedure is performed 
on the boundary (2-D), the unknowns of the problem when analysing a 3D structures are reduced drastically compared to those needed by FD or FE procedures. This approach has been applied to analyse different 3D passive microwave metallic structures demonstrating to be very efficient $[74,75]$. However, this method has been seldom applied to problems including dielectric obstacles, such as the full-wave analysis of H-plane filters with dielectric resonators [76]. Nowadays, in parallel to the work presented in this thesis, the research group of the University of Pavia has been also developing a different formulation to analyse rectangular waveguides loaded with dielectric resonators [77-79].

In this chapter we present the state-space integral-equation approach based on the BIRME method that we have developed to characterise a dielectric-loaded resonator cavity. As it is shown in the first section, the dielectric resonator has been rigorously characterized by means of the electric equivalent polarization charge and current densities defined in the volume of the dielectric object. Starting from the 'hybrid' representation of the field in a cavity resonator (presented in the previous chapter), we develop the SS-IE formulation in the second section. Following this method, the resonant modes of the considered cavities are obtained through the solution of a linear matrix eigenvalue problem in the third section. And finally, in the last section, the pole expansion of the generalized admittance matrix of the dielectric-loaded cavity is obtained in the domain of the Laplace variable.

\subsection{Dielectric obstacle characterization by means of the equiv- alent polarization currents}

It is well-known that dielectrics are materials whose dominant charges in atoms and molecules are bound negative and positive charges that are held in place by atoms and molecular forces, and they are not free to travel. Thus, ideal dielectrics are macroscopically neutral. However when external fields are applied, the centroides of these bound negative and positive charges can shift slightly in positions relative to each other, thus inducing a net electric dipole moment. Basically, there are three mechanisms that may produce electric polarization: Orientation polarization, which is produced in materials that are composed by polar molecules that carry a permanent dipole moment (polar molecules) orientated randomly, in such a way that the net dipole moment of the material in the absence of field is zero. When a field is applied, it tends to line up the individual molecular dipoles producing a net moment per unit volume. Electronic polarization is produced in materials whose molecules are nonpolar, so there are no individual dipoles in the absence of field. In this case, when an electric field is applied, a slight displacement of the centers of charge occurs, and a net dipole moment is induced. Ionic polarization in materials that possess positive and negative ions, is due to the displacement of these ions when an external field is applied, inducing a net moment. There is also a class of dielectric materials that are usually referred to as ferroelectrics. They exhibit a hysteresis loop of polarization $(\vec{P})$ versus electric field $(\vec{E})$ that is similar to the hysteresis loop of $\vec{B}$ versus $\vec{H}$ for ferromagnetic material, and it possesses a remanent polarization and 
coercive electric field.

Let consider a source-free region of space containing an inhomogeneity characterized by relative permittivity, which may be a function of position $\varepsilon_{r}=\varepsilon_{r}(\vec{r})$. If we consider a linear and isotropic medium and time-harmonic fields, the electromagnetic field in the vicinity of the inhomogeneity must satisfy the Maxwell's equations

$$
\begin{aligned}
& \nabla \times \vec{E}=-j \omega \mu_{o} \vec{H} \\
& \nabla \times \vec{H}=j \omega \varepsilon_{0} \varepsilon_{r} \vec{E} \\
& \nabla \cdot\left(\varepsilon_{0} \varepsilon_{r} \vec{E}\right)=0 \\
& \nabla \cdot\left(\mu_{o} \vec{H}\right)=0
\end{aligned}
$$

In order to calculate the electromagnetic field in a perfect conducting cavity resonator loaded with a dielectric body, one could try to solve directly the differential equations presented above or the corresponding integral equations. However, this task can be rather complicated. For this reason, it is convenient to convert the original scattering problem into an equivalent problem which may result a simpler one. One way of accomplishing this is to replace the inhomogeneous dielectric material present in the problem by equivalent induced polarization currents and charges, as we detail in the following paragraphs.

If an external electric field is applied to a linear and isotropic dielectric material, the induced polarization vector $\vec{P}$ (electric dipole moment per unit volume) inside the dielectric body is proportional to the total electric field applied [48]:

$$
\vec{P}(\vec{r})=\varepsilon_{0} \chi_{e}(\vec{r}) \vec{E}(\vec{r})=\varepsilon_{0}\left(\varepsilon_{r}(\vec{r})-1\right) \vec{E}(\vec{r})
$$

where $\chi_{e}(\vec{r})$ is the electric susceptibility, which may be a function of position.

Defining now, the displacement vector as:

$$
\vec{D}(\vec{r}) \equiv \varepsilon_{0} \vec{E}(\vec{r})+\vec{P}(\vec{r})
$$

and introducing the displacement vector in the Maxwell's divergence equation (3.1c), we have

$$
\nabla \cdot \vec{D}=\varepsilon_{0} \nabla \cdot \vec{E}+\nabla \cdot \vec{P}=0 \quad \Longrightarrow \quad \nabla \cdot \vec{E}=-\left(1 / \varepsilon_{0}\right) \nabla \cdot \vec{P}
$$

Thus, the term $-\nabla \cdot \vec{P}$ may be interpreted as defining an electric-polarization charge density $\rho_{p}$ :

$$
\rho_{p}=-\nabla \cdot \vec{P}
$$


On the other hand, by making use of the expression (3.2), the Maxwell's curl equation (3.1b), can be rewritten as follows:

$$
\begin{aligned}
\nabla \times \vec{H} & =j \omega \varepsilon_{0} \varepsilon_{r} \vec{E}=j \omega \varepsilon_{0} \varepsilon_{r} \vec{E}+j \omega \varepsilon_{0} \vec{E}-j \omega \varepsilon_{0} \vec{E} \\
& =j \omega \varepsilon_{0} \vec{E}+j \omega \varepsilon_{0}\left(\varepsilon_{r}-1\right) \vec{E}=j \omega \varepsilon_{0} \vec{E}+j \omega \vec{P}
\end{aligned}
$$

As a consequence, the term $\mathrm{\omega} \omega \vec{P}$ may be regarded as an equivalent polarization current density,

$$
\overrightarrow{J_{p}}=j \omega \vec{P}
$$

Furthermore, it can be easily verified that the equivalent polarization current and charge densities satisfy the continuity equation $\nabla \cdot \vec{J}_{p}+j \omega \rho_{p}=0$.

Next, by introducing (3.6) and (3.4) in the expressions (3.1), the Maxwell's equations can be rewritten:

$$
\begin{aligned}
& \nabla \times \vec{E}=-j \omega \mu_{o} \vec{H} \\
& \nabla \times \vec{H}=j \omega \varepsilon_{0} \vec{E}+\vec{J}_{p} \\
& \nabla \cdot\left(\varepsilon_{0} \vec{E}\right)=\rho_{p} \\
& \nabla \cdot\left(\mu_{o} \vec{H}\right)=0
\end{aligned}
$$

where $\overrightarrow{J_{p}}$ and $\rho_{p}$ are, respectively, the equivalent induced polarization currents and charges defined in equations (3.5) and (3.7).

Equations (3.8) describe the same electromagnetic fields as equations (3.1), but involve a homogeneous space characterized by permittivity $\varepsilon_{0}$ and permeability $\mu_{o}$ in which the equivalent charge and current densities radiate instead of the original heterogeneous environment. We can think of these equivalent sources as replacing the dielectric material in the original problem (3.1). Thus, we have defined a new and simpler problem which is completely equivalent to the original one. This procedure of replacing the dielectric material by induced sources is known as the volumetric equivalent principle [47].

Next, we should express the equivalent polarization charge $\rho_{p}$ in terms of the polarization vector $\vec{P}$. Due to the fact that the normal component of the displacement vector at the interface between the dielectric and the vacuum is a continuous function, it is firstly convenient to write $\vec{P}$ in terms of the displacement vector $\vec{D}$. By introducing equation (3.2) into (3.3), we obtain:

$$
\vec{P}(\vec{r})=\left(1-\frac{1}{\varepsilon_{r}(\vec{r})}\right) \vec{D}(\vec{r})
$$

Next, by making use of the equation (3.5), we can observe that the evaluation of $\rho_{p}$ is reduced to the calculation of the gradient of the function $1 / \varepsilon_{r}(\vec{r})$,

$$
\rho_{p}(\vec{r})=-\vec{D}(\vec{r}) \cdot \nabla\left(1-\frac{1}{\varepsilon_{r}(\vec{r})}\right)-\left(1-\frac{1}{\varepsilon_{r}(\vec{r})}\right) \nabla \cdot \vec{D}(\vec{r})=\vec{D}(\vec{r}) \cdot \nabla \frac{1}{\varepsilon_{r}(\vec{r})}
$$


where we have considered that free charges are not present in the dielectric body and therefore $\nabla \cdot \vec{D}=0$.

Up to now, we have considered the general case in which the dielectric resonator may be an heterogeneous material. Nevertheless, in most of the practical cases, it is assumed that the dielectric resonator material is homogeneous and non dispersive in the electromagnetic power range in which they are operative. In that case, by denoting as $\varepsilon_{r_{1}}$ the relative dielectric permittivity of the dielectric obstacle placed inside the cavity resonator, we may express the relative dielectric permittivity $\varepsilon_{r}(\vec{r})$ of the whole problem as a discontinuous function of the position vector:

$$
\varepsilon_{r}(\vec{r})=\left\{\begin{array}{cl}
\varepsilon_{r_{1}} & , \vec{r} \in V_{1} \\
1 & , \vec{r} \notin V_{1}
\end{array}\right.
$$

where $V_{1}$ is the volume of the dielectric inset immersed in the cavity resonator, $S_{1}$ is its surface (the interface between the vacuum and the dielectric material), and $\widehat{n}_{1}$ is the unitary outward normal vector to $S_{1}$.

To calculate $\nabla \varepsilon_{r}^{-1}$, we may rewrite equation (3.11) in a general orthogonal coordinate system as:

$$
\varepsilon_{r}(\vec{r})=\varepsilon_{r}\left(n_{1}\right)=\varepsilon_{r_{1}}+\left(1-\varepsilon_{r_{1}}\right) H\left(n_{1}-n_{1_{d}}\right)
$$

where $n_{1}$ denotes the normal outward coordinate employed to define the boundary of the dielectric object and $H\left(n_{1}-n_{1_{d}}\right)$ is the unit Heaviside function or step function, defined as:

$$
H(x)= \begin{cases}0 & , x<0 \\ 1 & , x \geq 0\end{cases}
$$

Note that with this notation, $n_{1}=n_{1_{d}}$ sets the interface between vacuum and the dielectric (surface $S_{1}$ ), so the explicit form of the Heaviside function is:

$$
H\left(n_{1}-n_{1_{d}}\right)= \begin{cases}0 & , \vec{r} \in V_{1} \\ 1 & , \vec{r} \notin V_{1}\end{cases}
$$

Hence, we can also write:

$$
\varepsilon_{r}^{-1}(\vec{r})=\varepsilon_{r}^{-1}\left(n_{1}\right)=\varepsilon_{r_{1}}^{-1}+\left(1-\varepsilon_{r_{1}}^{-1}\right) H\left(n_{1}-n_{1_{d}}\right)
$$

The expression of the gradient operator in the general coordinate system is:

$$
\nabla \varepsilon_{r}(\vec{r})=\sum_{i=1}^{3} \frac{\widehat{x}_{i}}{h_{i}} \frac{\partial \varepsilon_{r}}{\partial x_{i}}=\frac{\widehat{n}_{1}}{h_{n_{1}}} \frac{\partial \varepsilon_{r}\left(n_{1}\right)}{\partial n_{1}}
$$

where $h_{i}$ is the metrical coefficient.

Now, we apply the operator to expression (3.14), obtaining

$$
\nabla \frac{1}{\varepsilon_{r}(\vec{r})}=\left(1-\frac{1}{\varepsilon_{r_{1}}}\right) \delta\left(n_{1}-n_{1_{d}}\right) \frac{\widehat{n}_{1}}{h_{n_{1}}}
$$


where $\delta$ is the Dirac delta function. Finally, if we introduce this last expression into (3.10), we have

$$
\rho_{p}(\vec{r})=\left(\vec{D} \cdot \widehat{n}_{1}\right) \frac{1}{h_{n_{1}}}\left(1-\frac{1}{\varepsilon_{r_{1}}}\right) \delta\left(n_{1}-n_{1_{d}}\right)=\left(\vec{P} \cdot \widehat{n}_{1}\right) \frac{1}{h_{n_{1}}} \delta\left(n_{1}-n_{1_{d}}\right)
$$

This equation implies that the volumetric equivalent electric charge density is actually distributed on the surface of the dielectric body $S_{1}$ : it is a surface equivalent polarization charge density rather than a volumetric one, as follows:

$$
\rho_{p}(\vec{r})=\left\{\begin{array}{cl}
\frac{1}{h_{n_{1}}} P_{n_{1}} & , \vec{r} \in S_{1} \\
0 & , \vec{r} \notin S_{1}
\end{array}\right.
$$

Note that $P_{n_{1}}$ is the normal component of the polarization vector $\vec{P}$ to the surface $S_{1}$.

In conclusion, the volumetric equivalence principle allows us to rigorously replace the isotropic, linear and homogeneous dielectric material by:

- Surface equivalent polarization charge density distributed on the interface between the dielectric and vacuum: $\rho_{p}$

- Volume equivalent polarization current density defined in the volume of the dielectric body: $\overrightarrow{J_{p}}$

As a consequence, the solution of the original scattering problem is equivalent to finding the electromagnetic fields generated by these polarization sources radiating in vacuum. For such purpose, expressions (3.7) and (3.17), which give the equivalent polarization charge $\rho_{p}$ and current $\overrightarrow{J_{p}}$ densities as functions of the polarization vector $\vec{P}$, will be useful in the next section to solve the integral equations corresponding to the new equivalent problem.

\subsection{State-Space Integral-Equation Approach}

The building block under study is a linear, homogeneous, non-dispersive and isotropic dielectric inset arbitrarily placed inside a resonator cavity with perfect conductive walls. The volume of the dielectric inset is denoted by $V_{1}$ and its surface by $S_{1}$, whereas the volume of the cavity resonator is $V$ and its surface $S$, which partially coincides with the surfaces of the access ports $S_{\nu}$. In Fig. 3.1, a diagram of the building block is plotted. Note that, for the sake of simplicity, a rectangular cavity with two access ports has been drawn. Nevertheless, the cavity resonator may have a shape different from the rectangular one, and the number of access ports may be unlimited.

The electromagnetic behaviour of this circuit building block can be characterized by means of its generalized admittance matrix, which relates the modal voltages $v_{n}$ and the modal currents $i_{n}$ defined at each access port of the cavity resonator. If we consider a number 
of accessible modes $N_{\nu}$ at each access port $(\nu)$, then the total number of accessible modes is $N=\sum_{\nu} N_{\nu}$, and the GAM will be the following $N \times N$ matrix:

$$
Y_{m, n}=\frac{i_{m}}{v_{n}} ; \quad \text { with } \quad v_{l}=0 \quad \forall l \neq n
$$

In order to simplify the notation of the problem we will consider the modal vectors to be defined in the whole surface of the resonator cavity $S$, being zero outside the surface of its correspondent waveguide port $S_{\nu}$. Thus, if we order the different modal vectors from each access port, we can denote:

$$
\vec{e}_{n}(\vec{r}) \equiv \vec{e}_{i}^{(\nu)}(\vec{r}) \delta^{(\nu)}(\vec{r})
$$

where $n=1, \cdots, N$ and $i=1, \cdots, N_{\nu}$. The delta function is defined as:

$$
\delta^{(\nu)}(\vec{r}) \equiv \begin{cases}1 & \forall \vec{r} \in S_{\nu} \\ 0 & \forall \vec{r} \notin S_{\nu}\end{cases}
$$

The same relationship holds for the magnetic modal vectors $\vec{h}_{n}$. The electric and magnetic modal vectors $\vec{e}_{n}^{(\nu)}$ and $\vec{h}_{n}^{(\nu)}$ defined at the access waveguides are normalized, therefore we can write:

$$
\int_{s_{\nu}} \vec{e}_{m} \cdot \vec{e}_{n} d s=\int_{s_{\nu}} \vec{h}_{m} \cdot \vec{h}_{n} d s=\delta_{m n}
$$

where $\delta$ is the Kronecker delta function. Hence, the following relationship holds:

$$
\vec{h}_{n}=\widehat{n} \times \vec{e}_{n}
$$

where $\widehat{n}$ is the inward normal to $S$.

With these definitions, the tangential electric field over the boundary of the cavity can be expressed in terms of the normalized waveguide electric modal vectors,

$$
\vec{E}_{\text {tang }}=\sum_{\nu} \sum_{n=1}^{N_{\nu}} v_{n} \vec{e}_{n}^{(\nu)}=\sum_{n=1}^{N} v_{n} \vec{e}_{n},
$$

and the tangential magnetic field over the boundary of the cavity as,

$$
\vec{H}_{\text {tang }}=\sum_{\nu} \sum_{n=1}^{N_{\nu}} i_{n} \vec{h}_{n}^{(\nu)}=\sum_{n=1}^{N} i_{n} \vec{h}_{n},
$$

Thus, the modal current $i_{n}$ defined at each port is related to the tangential magnetic field $\vec{H}_{\text {tang }}$ as follows

$$
i_{n}=\int_{S} \vec{h}_{n} \cdot \vec{H}_{t a n g} d s
$$




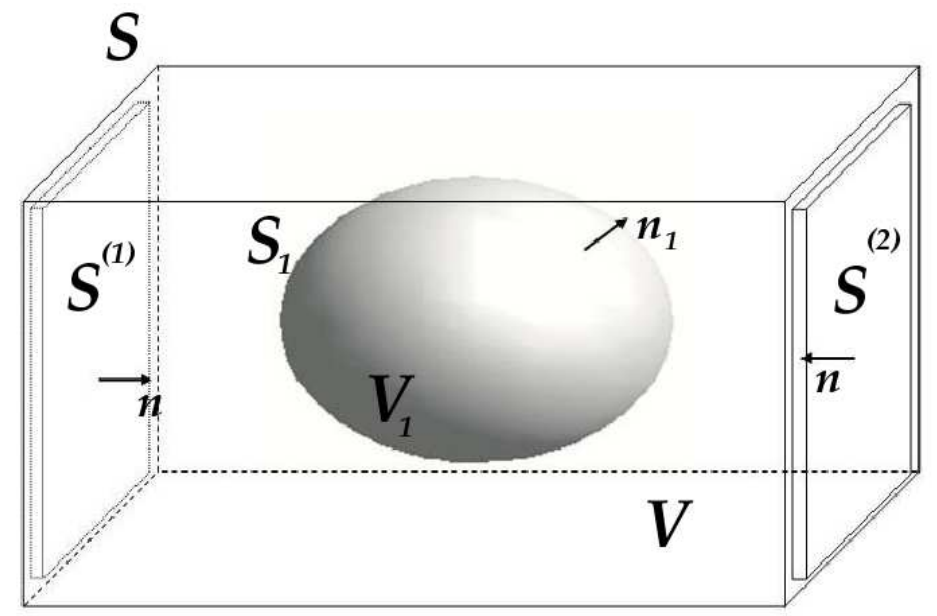

Figure 3.1: The basic building block under study: a dielectric resonator arbitrarily placed inside a cavity resonator connected to access ports.

In order to determine the generalized admittance matrix of the structure under analysis, the functional relationship between the tangential magnetic $\left(\vec{H}_{T}\right)$ and electric $\left(\vec{E}_{T}\right)$ fields over the cavity access ports must be found.

Taking us starting point the 'hybrid' representation of the field (2.88), we can express the electromagnetic field in a finite region generated by inner volumetric electric sources and magnetic current sheets ${ }^{4}$ on the boundary in terms of the electric scalar and vector potentials (in the Coulomb gauge):

$$
\begin{aligned}
\vec{E}(\vec{r}) & =\frac{\eta}{s} \nabla \int_{V} g^{e}\left(\vec{r}, \vec{r}^{\prime}\right) \nabla^{\prime} \cdot \vec{J}\left(\vec{r}^{\prime}\right) d v^{\prime}+\frac{1}{2} \widehat{n} \times \vec{M}(\vec{r}) \\
& -s \eta \int_{V} \underline{G}_{o}^{A}\left(\vec{r}, \vec{r}^{\prime}\right) \cdot \vec{J}\left(\vec{r}^{\prime}\right) d v^{\prime}-\int_{S} \nabla \times \underline{G}_{o}^{F}\left(\vec{r}, \vec{r}^{\prime}\right) \cdot \vec{M}\left(\vec{r}^{\prime}\right) d s^{\prime} \\
& +s^{3} \eta \sum_{m=1}^{M} \frac{\int_{V} \vec{E}_{m} \cdot \vec{J} d v}{k_{m}^{2}\left(s^{2}+k_{m}^{2}\right)} \vec{E}_{m}(\vec{r})+s^{2} \sum_{m=1}^{M} \frac{\int_{S} \vec{H}_{m} \cdot \vec{M} d s}{k_{m}\left(s^{2}+k_{m}^{2}\right)} \vec{E}_{m}(\vec{r}) \\
\vec{H}(\vec{r}) & =\frac{1}{s \eta} \nabla \int_{S} g^{m}\left(\vec{r}, \vec{r}^{\prime}\right) \nabla_{s}^{\prime} \cdot \vec{M}\left(\vec{r}^{\prime}\right) d s^{\prime} \\
& -\frac{s}{\eta} \int_{S} \underline{G}_{o}^{F}\left(\vec{r}, \vec{r}^{\prime}\right) \cdot \vec{M}\left(\vec{r}^{\prime}\right) d s^{\prime}+\int_{V} \nabla \times \underline{G}_{o}^{A}\left(\vec{r}, \vec{r}^{\prime}\right) \cdot \vec{J}\left(\vec{r}^{\prime}\right) \\
& +\frac{s^{3}}{\eta} \sum_{m=1}^{M} \frac{\int_{S} \vec{H}_{m} \cdot \vec{M} d s}{k_{m}^{2}\left(s^{2}+k_{m}^{2}\right)} \vec{H}_{m}(\vec{r})-s^{2} \sum_{m=1}^{M} \frac{\int_{V} \vec{E}_{m} \cdot \vec{J} d v}{k_{m}\left(s^{2}+k_{m}^{2}\right)} \vec{H}_{m}(\vec{r})
\end{aligned}
$$

\footnotetext{
${ }^{4}$ In the expressions (2.88), we have considered volumetric source currents distributed in the cavity. Nevertheless, wether current sheets are considered, it is important to study the last term in the right hand side of equations (2.86) and (2.87). The singularity of $\nabla \times \underline{G}^{A}$ or $\nabla \times \underline{G}^{F}$ is of the order $R^{-2}$ which is integrable when we perform a volumetric integration, but it is not when the integration is performed over a surface. The proper treatment of the singularity yields to the new term $(1 / 2) \widehat{n} \times \vec{M}(\vec{r})$ in the case of magnetic currents [73].
} 
where $\nabla_{s}$ is the surface divergence operator.

We recall, from sections 2.4 and 2.6, that in these expressions $g^{e}, g^{m}$ are electric and magnetic static scalar potential Green's functions, and $\underline{G}_{o}^{A}, \underline{G}_{o}^{F}$ are, respectively, the static dyadic Green's functions for the electric and magnetic vector potentials of the resonant cavity ${ }^{5}$. Whereas $k_{m}, \vec{E}_{m}$ and $\vec{H}_{m}$ are, respectively, the resonant wavenumber, the normalized electric and magnetic modal vector functions of the $m$-th mode of the resonator cavity. As it was discussed in the previous chapter, the resonant mode expansions converges very quickly, and can be truncated by retaining the resonant modes that satisfy $k_{m} \leq \xi k_{\max }$, where $k_{\max }$ is the wavenumber correspondent to the highest frequency in the band of interest, and $\xi$ is an accuracy factor.

In the present formulation, the magnetic sources represent impressed current sheets defined in the waveguide cross-section of the access ports connected to the cavity [85],

$$
\vec{M}(\vec{r})=-\widehat{n} \times \vec{E}_{\text {tang }}(\vec{r})=-\widehat{n} \times \sum_{n=1}^{N} v_{n} \vec{e}_{n}(\vec{r})=-\sum_{n=1}^{N} v_{n} \vec{h}_{n}(\vec{r})
$$

With regard to the electric sources, as it has been discussed in the previous section, we may replace the homogeneous dielectric inset with dielectric permittivity $\varepsilon_{r_{1}}$ by its equivalent polarization charge $\rho_{p}$ and current $\vec{J}_{p}$ densities radiating inside the resonator cavity. Thus, we introduce equations (3.7), (3.17) and (3.28) into (3.27), and taking into account that

$$
\begin{aligned}
& -\int_{V} g^{e}\left(\vec{r}, \vec{r}^{\prime}\right) \nabla^{\prime} \cdot \vec{J}_{p}\left(\vec{r}^{\prime}\right)=j \omega \int_{V} g^{e}\left(\vec{r}, \vec{r}^{\prime}\right) \rho_{p}\left(\vec{r}^{\prime}\right) \\
& \quad=\int_{V} g^{e}\left(\vec{r}, \vec{r}^{\prime}\right) \vec{P}\left(\vec{r}^{\prime}\right) \cdot \widehat{n}_{1} \delta\left(n_{1}-n_{1_{d}}\right) d v=\int_{S_{1}} g^{e}\left(\vec{r}, \vec{r}^{\prime}\right) \vec{P}\left(\vec{r}^{\prime}\right) \cdot \widehat{n}_{1} d s^{\prime}
\end{aligned}
$$

\footnotetext{
${ }^{5}$ We could have used the free space Green's functions instead of these ones, which have simpler expressions than those for a rectangular or a spherical resonator. Nevertheless, it would yield to a non-linear matrix eigenvalue problem that should be solved over and over again at each frequency point. In addition, since we use the Green's function of the resonator cavity, the boundary conditions in the metal walls of the cavity are directly fulfilled.
} 
we obtain:

$$
\begin{aligned}
\vec{E}(\vec{r}) & =-\frac{1}{\varepsilon_{0}} \nabla \int_{S_{1}} g^{e}\left(\vec{r}, \vec{r}^{\prime}\right) \vec{P}\left(\vec{r}^{\prime}\right) \cdot \widehat{n}_{1} d s^{\prime}-\frac{s^{2}}{\varepsilon_{0}} \int_{V} \underline{G}_{o}^{A}\left(\vec{r}, \vec{r}^{\prime}\right) \cdot \vec{P}\left(\vec{r}^{\prime}\right) d v^{\prime} \\
& +\sum_{n=1}^{N} v_{n} \int_{S} \nabla \times \underline{G}_{o}^{F}\left(\vec{r}, \vec{r}^{\prime}\right) \cdot \vec{h}_{n}\left(\vec{r}^{\prime}\right) d s^{\prime}+s^{2} \sum_{m=1}^{M} a_{m} \vec{E}_{m}(\vec{r}) \\
& +\frac{1}{2} \sum_{n=1}^{N} v_{n} \vec{e}_{n}(\vec{r}) \\
\vec{H}(\vec{r}) & =-\frac{1}{s \eta} \sum_{n=1}^{N} v_{n} \nabla \int_{S} g^{m}\left(\vec{r}, \vec{r}^{\prime}\right) \nabla_{s}^{\prime} \cdot \vec{h}_{n}\left(\vec{r}^{\prime}\right) d s^{\prime} \\
& +\frac{s}{\eta} \sum_{n=1}^{N} v_{n} \int_{S} \underline{G}_{o}^{F}\left(\vec{r}, \vec{r}^{\prime}\right) \cdot \vec{h}_{n}\left(\vec{r}^{\prime}\right) d s^{\prime}+c s \int_{V} \nabla \times \underline{G}_{o}^{A}\left(\vec{r}, \vec{r}^{\prime}\right) \cdot \vec{P}\left(\vec{r}^{\prime}\right) \\
& -\frac{s}{\eta} \sum_{m=1}^{M} k_{m} a_{m} \vec{H}_{m}(\vec{r})-\frac{s}{\eta} \sum_{n=1}^{N} v_{n} \sum_{m=1}^{M} \frac{\int_{S} \vec{H}_{m} \cdot \vec{h}_{n} d s}{k_{m}^{2}} \vec{H}_{m}(\vec{r})
\end{aligned}
$$

where the modal amplitudes $a_{m}$ have been defined as:

$$
a_{m} \equiv \frac{1}{k_{m}^{2}\left(s^{2}+k_{m}^{2}\right)}\left(s \eta \int_{V_{1}} \vec{E}_{m}(\vec{r}) \cdot \vec{J}(\vec{r}) d v-k_{m} \sum_{n=1}^{N} v_{n} \int_{S} \vec{H}_{m}(\vec{r}) \cdot \vec{h}_{n}(\vec{r}) d s\right)
$$

Next, by imposing (3.2) in the dielectric volume $V_{1}$ using (3.29a), and expressing the tangential magnetic field on the boundary of the resonator cavity in terms of the total set of sources using (3.29b), we obtain the following integral equations:

$$
\begin{aligned}
\vec{P}(\vec{r})= & -\chi_{e_{1}}\left[\nabla \int_{S_{1}} g^{e}\left(\vec{r}, \vec{r}^{\prime}\right) \vec{P}\left(\vec{r}^{\prime}\right) \cdot \widehat{n}_{1} d s^{\prime}+s^{2} \int_{V_{1}} \underline{G}_{o}^{A}\left(\vec{r}, \vec{r}^{\prime}\right) \cdot \vec{P}\left(\vec{r}^{\prime}\right) d v^{\prime}\right. \\
& \left.-\varepsilon_{0}\left(\sum_{n=1}^{N} v_{n} \int_{S} \nabla \times \underline{G}_{o}^{F}\left(\vec{r}, \vec{r}^{\prime}\right) \cdot \vec{h}_{n}\left(\vec{r}^{\prime}\right) d s^{\prime}+s^{2} \sum_{m=1}^{M} a_{m} \vec{E}_{m}(\vec{r})\right)\right] ; \quad \vec{r} \in V_{1} \\
\vec{H}_{T}(\vec{r})= & -\frac{1}{s \eta} \sum_{n=1}^{N} v_{n} \nabla_{s} \int_{S} g^{m}\left(\vec{r}, \vec{r}^{\prime}\right) \nabla_{s}^{\prime} \cdot \vec{h}_{n}\left(\vec{r}^{\prime}\right) d s^{\prime} \\
& +\left(\frac{s}{\eta} \sum_{n=1}^{N} v_{n} \int_{S} \underline{G}_{o}^{F}\left(\vec{r}, \vec{r}^{\prime}\right) \cdot \vec{h}_{n}\left(\vec{r}^{\prime}\right) d s^{\prime}+s c \int_{V_{1}} \nabla \times \underline{G}_{o}^{A}\left(\vec{r}, \vec{r}^{\prime}\right) \cdot \vec{P}\left(\vec{r}^{\prime}\right) d v^{\prime}\right. \\
& \left.-\frac{s}{\eta} \sum_{n=1}^{N} v_{n} \sum_{m=1}^{M} \frac{\int_{S} \vec{H}_{m} \cdot \vec{h}_{n} d s}{k_{m}^{2}} \vec{H}_{m}(\vec{r})-\frac{s}{\eta} \sum_{m=1}^{M} k_{m} a_{m} \vec{H}_{m}(\vec{r})\right)_{T} ; \quad \vec{r} \in S
\end{aligned}
$$

where the subindex $T$ indicates tangential. We notice that in expressions (3.31) we can observe the characteristic structure of the BI-RME solutions: frequency-independent kernels 
made up of boundary integrals (BI) and resonant mode expansions (RME). However, in our case we do not have boundary, but volume integrals.

In order to obtain a numerical solution for the previous set of integral equations (3.31), we apply the well-known Method of Moments (MoM) [46]. Thus, the unknown polarization density is approximated as follows,

$$
\vec{P}(\vec{r}) \approx \varepsilon_{0} \chi_{e_{1}} \sum_{q=1}^{Q} b_{q} \vec{w}_{q}(\vec{r}) ; \quad \vec{r} \in V_{1}
$$

where $b_{q}$ are unknown coefficients, $\left\{\vec{w}_{q}\right\}$ are a set of basis functions defined in $V_{1}$ and $Q$ is the number of basis functions used in the expansion [62]. These basis functions have to satisfy the following properties:

1. $\left\{\vec{w}_{q}\right\}$ are continuous in $V_{1}$.

2. Since the divergence of $\vec{P}$ is zero for the points within the dielectric inset (section 3.2), then $\left\{\vec{w}_{q}\right\}$ are solenoidal functions:

$$
\nabla \cdot \vec{w}_{q}(\vec{r})=0 ; \quad \vec{r} \in V_{1}
$$

The next step in the formulation is to solve the electric field integral equation (EFIE) by means of the MoM using the Galerkin's method. For this purpose, we substitute (3.32) in (3.31a), and using as testing functions the set of basis functions $\left\{\vec{w}_{p}\right\}$, we can write:

$$
\begin{aligned}
\sum_{q=1}^{Q} b_{q} \int_{V_{1}} \vec{w}_{p}(\vec{r}) \cdot \vec{w}_{q}(\vec{r}) d v= & -\chi_{e_{1}} \sum_{q=1}^{Q} b_{q} \int_{S_{1}} \int_{S_{1}}\left(\vec{w}_{p}(\vec{r}) \cdot \widehat{n}_{1}\right) g^{e}\left(\vec{r}, \vec{r}^{\prime}\right)\left(\vec{w}_{q}\left(\vec{r}^{\prime}\right) \cdot \widehat{n}_{1}\right) d s^{\prime} d s \\
& -\chi_{e_{1}} s^{2} \sum_{q=1}^{Q} b_{q} \int_{V_{1}} \int_{V_{1}} \vec{w}_{p}(\vec{r}) \cdot \underline{G}_{o}^{A}\left(\vec{r}, \vec{r}^{\prime}\right) \cdot \vec{w}_{q}\left(\vec{r}^{\prime}\right) d v^{\prime} d v \\
& +\sum_{n=1}^{N} v_{n} \int_{V_{1}} \int_{S} \vec{w}_{p}(\vec{r}) \cdot \nabla \times \underline{G}_{o}^{F}\left(\vec{r}, \vec{r}^{\prime}\right) \cdot \vec{h}_{n}\left(\vec{r}^{\prime}\right) d s^{\prime} d v \\
& +s^{2} \sum_{m=1}^{M} a_{m} \int_{V_{1}} \vec{w}_{p}(\vec{r}) \cdot \vec{E}_{m}(\vec{r}) d v
\end{aligned}
$$

where $p=1,2, \ldots, Q$. Note that in order to obtain the first term in the right hand side of equation (3.34), we may define a regular function

$$
\vartheta(\vec{r}) \equiv \int_{S_{1}} g^{e}\left(\vec{r}, \vec{r}^{\prime}\right)\left(\vec{w}_{q}\left(\vec{r}^{\prime}\right) \cdot \widehat{n}_{1}\right) d s^{\prime}
$$

and since $\vec{w}_{q}$ is solenoidal, then using the divergence theorem we have

$$
\begin{aligned}
\int_{V_{1}} \vec{w}_{p}(\vec{r}) \cdot \nabla \vartheta(\vec{r}) d v= \\
=\int_{S_{1}}\left(\vec{w}_{p}(\vec{r}) \cdot \widehat{n}_{1}\right) \vartheta(\vec{r}) d s=\int_{S_{1}} \int_{S_{1}}\left(\vec{w}_{p}(\vec{r}) \cdot \widehat{n}_{1}\right) g^{e}\left(\vec{r}, \vec{r}^{\prime}\right)\left(\vec{w}_{q}\left(\vec{r}^{\prime}\right) \cdot \widehat{n}_{1}\right) d s^{\prime} d s
\end{aligned}
$$


Expression (3.34) involves $Q+M$ unknown coefficients $b_{q}$ and $a_{m}$, whereas it implies only $Q$ equations. Thus, to find the final system of equations that will provide us the solution of the problem, we need $M$ more equations that we obtain by substituting (3.32) in (3.30),

$$
\left(k_{m}^{4}+s^{2} k_{m}^{2}\right) a_{m}=s^{2} \chi_{e_{1}} \sum_{q=1}^{Q} b_{q} \int_{V_{1}} \vec{E}_{m} \cdot \vec{w}_{q} d v-k_{m} \sum_{n=1}^{N} v_{n} \int_{S} \vec{H}_{m} \cdot \vec{h}_{n} d s
$$

where $m=1,2, \ldots, M$ are the number of resonant modes used in the expansion of the Green's function.

Finally, making use of expressions (3.34) and (3.35) we can transform the integral equations into a system of coupled linear algebraic equations:

$$
\begin{gathered}
\mathbf{K}^{4} \mathbf{a}+s^{2} \mathbf{K}^{2} \mathbf{a}-s^{2} \chi_{e_{1}} \mathbf{S} \mathbf{b}=-\mathbf{K} \mathbf{F} \mathbf{v} \\
\left(\mathbf{D}+\chi_{e_{1}} \mathbf{C}\right) \mathbf{b}-s^{2} \mathbf{S}^{T} \mathbf{a}+s^{2} \chi_{e_{1}} \mathbf{L} \mathbf{b}=\mathbf{W} \mathbf{v}
\end{gathered}
$$

where $\mathbf{a}$ and $\mathbf{b}$ are, respectively, vectors with the modal amplitudes $a_{m}$ and the $b_{q}$ coefficients, $\mathbf{v}$ is the vector of the impressed modal voltages at the waveguide access ports, and the involved matrices are defined in Table 3.1. Now, defining the following matrices:

$$
\begin{array}{cc}
\mathbf{x} \equiv\left[\begin{array}{l}
\mathbf{a} \\
\mathbf{b}
\end{array}\right] ; & \mathbf{U} \equiv\left[\begin{array}{c}
-\mathbf{K F} \\
\chi_{e_{1}} \mathbf{W}
\end{array}\right] ; \\
\mathbf{A} \equiv\left[\begin{array}{cc}
\mathbf{K}^{4} & 0 \\
0 & \chi_{e_{1}}\left[\mathbf{D}+\chi_{e_{1}} \mathbf{C}\right]
\end{array}\right] ; & \mathbf{B} \equiv\left[\begin{array}{cc}
\mathbf{K}^{2} & -\chi_{e_{1}} \mathbf{S} \\
-\chi_{e_{1}} \mathbf{S}^{T} & \chi_{e_{1}}^{2} \mathbf{L}
\end{array}\right]
\end{array}
$$

we can re-write the system of equations defined by (3.36) in a compact form:

$$
\left(\mathbf{A}+s^{2} \mathbf{B}\right) \mathbf{x}=\mathbf{U} \mathbf{v}
$$

On the other hand, in order to calculate the generalized admittance matrix, we need to obtain a functional relationship between $\vec{H}_{T}$ and $\vec{E}_{T}$. For this purpose, we apply the continuity of the tangential magnetic field in the apertures of the cavity by imposing (3.25) in (3.31b), and then we introduce (3.32). Finally, we apply the MoM using as testing function the modal vectors $\left\{\vec{h}_{l}\right\}$. Thus, we have

$$
\begin{aligned}
i_{l}= & \frac{1}{s \eta} \sum_{n=1}^{N} v_{n} \int_{S} \int_{S}\left(\nabla_{s} \cdot \vec{h}_{l}(\vec{r})\right) g^{m}\left(\vec{r}, \vec{r}^{\prime}\right)\left(\nabla_{s}^{\prime} \cdot \vec{h}_{n}\left(\vec{r}^{\prime}\right)\right) d s^{\prime} \\
& +\frac{s}{\eta}\left(\sum_{n=1}^{N} v_{n} \int_{S} \int_{S} \vec{h}_{l}(\vec{r}) \cdot \underline{G}_{o}^{F}\left(\vec{r}, \vec{r}^{\prime}\right) \cdot \vec{h}_{n}\left(\vec{r}^{\prime}\right) d s^{\prime}\right. \\
& \quad+\chi_{e_{1}} \sum_{q=1}^{Q} b_{q} \int_{S} \int_{V_{1}} \vec{h}_{l}(\vec{r}) \cdot \nabla \times \underline{G}_{o}^{A}\left(\vec{r}, \vec{r}^{\prime}\right) \cdot \vec{w}_{q}\left(\vec{r}^{\prime}\right) d v^{\prime} \\
- & \left.\sum_{n=1}^{N} v_{n} \sum_{m=1}^{M} \frac{\int_{S} \vec{h}_{l}(\vec{r}) \cdot \vec{H}_{m}(\vec{r}) d s \int_{S} \vec{H}_{m}\left(\vec{r}^{\prime}\right) \cdot \vec{h}_{n}\left(\vec{r}^{\prime}\right) d s^{\prime}}{k_{m}^{2}}-\sum_{m=1}^{M} k_{m} a_{m} \int_{S} \vec{h}_{l}(\vec{r}) \cdot \vec{H}_{m}(\vec{r}) d s\right)
\end{aligned}
$$


where $l=1,2, \ldots, N$. Note that in order to obtain the first term in the r.h.s. of equation (3.39), we have made use of the relation

$$
\vec{h}_{l}(\vec{r}) \cdot \nabla_{s} g^{m}\left(\vec{r}, \vec{r}^{\prime}\right)=\nabla_{s} \cdot\left(g^{m}\left(\vec{r}, \vec{r}^{\prime}\right) \vec{h}_{l}(\vec{r})\right)-g^{m}\left(\vec{r}, \vec{r}^{\prime}\right) \nabla_{s} \cdot \vec{h}_{l}(\vec{r})
$$

Since the vector $g^{m}\left(\vec{r}, \vec{r}^{\prime}\right) \vec{h}_{l}(\vec{r})$ is tangential to $S$, and $S$ is a closed surface, using the divergence theorem on a surface [85], we have

$$
\int_{S} \int_{S} \nabla_{s} \cdot\left(g^{m}\left(\vec{r}, \vec{r}^{\prime}\right) \vec{h}_{l}(\vec{r})\right) d s^{\prime} d s^{\prime}=0
$$

and then

$$
\int_{S} \int_{S} \vec{h}_{l}(\vec{r}) \cdot \nabla_{s} g^{m}\left(\vec{r}, \vec{r}^{\prime}\right) d s^{\prime} d s^{\prime}=-\int_{S} \int_{S}\left(\nabla_{s} \cdot \vec{h}_{l}(\vec{r})\right) \cdot g^{m}\left(\vec{r}, \vec{r}^{\prime}\right) d s^{\prime} d s^{\prime}
$$

By making use of the matrices defined in expressions(3.37) and in Table 3.1, we can represent the integral equations (3.39) in matrix form:

$$
\mathbf{i}=\frac{1}{s \eta} \mathbf{G} \mathbf{v}+\frac{s}{\eta} \mathbf{U}^{T} \mathbf{x}+\frac{s}{\eta}\left(\mathbf{T}-\mathbf{F}^{T} \mathbf{K}^{-2} \mathbf{F}\right) \mathbf{v}
$$

Finally, we obtain the generalized admittance matrix $(i=Y v)$ by introducing equation (3.38) in (3.40):

$$
\mathbf{Y}=\frac{1}{s \eta} \mathbf{G}+\frac{s}{\eta}\left(\mathbf{T}-\mathbf{F}^{T} \mathbf{K}^{-2} \mathbf{F}\right)+\frac{s}{\eta} \mathbf{U}^{T}\left(\mathbf{A}+s^{2} \mathbf{B}\right)^{-1} \mathbf{U}
$$

\subsection{Resonant modes of the dielectric-loaded cavity}

To calculate the generalized admittance matrix by means of expression (3.41), it is needed to invert the matrix $\left(\mathbf{A}+s^{2} \mathbf{B}\right)$. This matrix is singular when $k$ is the eigenvalue of the homogeneous eigenvalue problem

$$
\left(\mathbf{A}-k^{2} \mathbf{B}\right) \mathbf{x}=0
$$

It can be observed that, if the impressed voltage is zero $(\mathbf{v}=0)$, then the equation (3.38) is casted into the homogeneous eigenvalue problem in (3.42). Thus, the expression (3.42) represents the problem of obtaining the resonant modes of the dielectric loaded cavity when the access ports to the cavity are closed by perfect electric wall. The eigenvalues give the resonant frequency of the structure under study, whereas the eigenvectors are directly related to the modal vectors existing in the resonant structure. Furthermore, the matrices $\mathbf{A}$ and $\mathbf{B}$ are positive definte ${ }^{6}$, and thus the solution of the eigenvalue problem yields to $(Q+M)$ real

\footnotetext{
${ }^{6}$ In [63] it is demonstrated that the matrices $\mathbf{A}$ and $\mathbf{B}$ are positive definte. In that work, the name of the two matrices are interchanged.
} 
Table 3.1: Definition of the Matrices Used in the Method of Moments

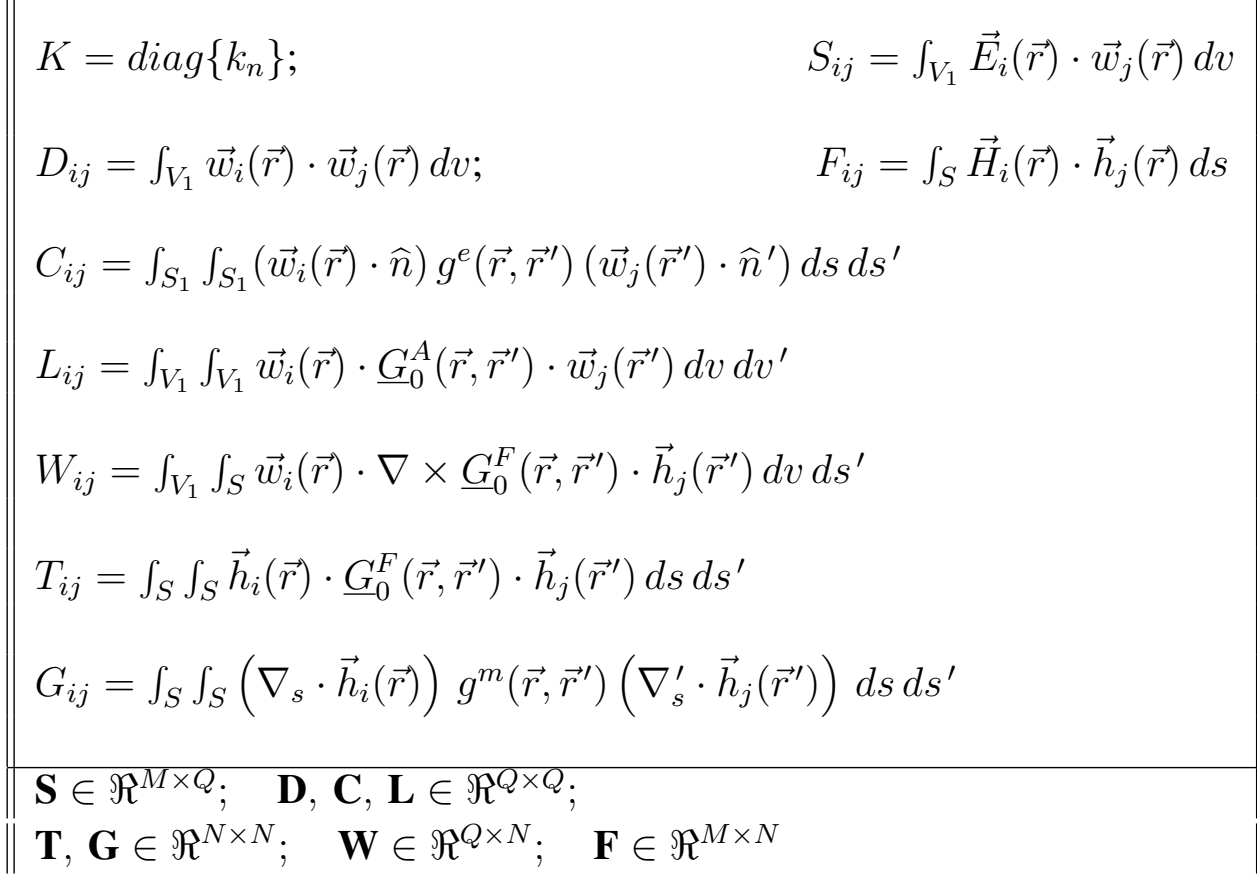

and positive eigenvalues $k_{i}^{2}$ and $(Q+M)$ real eigenvectors $\mathbf{x}^{(i)}$ (the normalization condition $\mathbf{x}_{T}^{(i)} \mathbf{B} \mathbf{x}^{(i)}=1$ is assumed), where the index $i$ denotes the $\mathrm{i}$-th resonant mode of the structure. We recall that:

$$
\mathbf{x}^{(i)} \equiv\left[\begin{array}{l}
\mathbf{a}^{(i)} \\
\mathbf{b}^{(i)}
\end{array}\right]
$$

Hence, by introducing the solution of this eigenvalue problem into (3.29) and making use of (3.32), we can obtain the field distribution of the i-th resonant mode of the dielectric loaded cavity resonator as:

$$
\begin{aligned}
& \vec{E}^{(i)}(\vec{r})=\chi_{e_{1}} \sum_{q}^{Q} b_{q}^{(i)}\left(\vec{F}_{q}(\vec{r})+k_{i}^{2} \vec{A}_{q}(\vec{r})\right)-k_{i}^{2} \sum_{m=1}^{M} a_{m}^{(i)} \vec{E}_{m}(\vec{r}) \\
& \vec{H}^{(i)}(\vec{r})=\frac{1}{\eta}\left(\chi_{e_{1}} k_{i} \sum_{q}^{Q} b_{q}^{(i)} \vec{B}_{q}(\vec{r})-k_{i} \sum_{m=1}^{M} a_{m}^{(i)} \vec{H}_{m}(\vec{r})\right)
\end{aligned}
$$

where the following definitions have been used:

$$
\begin{aligned}
\vec{F}_{q}(\vec{r}) & \equiv-\nabla \int_{S_{1}} g^{e}\left(\vec{r}, \vec{r}^{\prime}\right) \vec{w}_{q}\left(\vec{r}^{\prime}\right) \cdot \widehat{n}_{1} d s^{\prime} \\
\vec{A}_{q}(\vec{r}) & \equiv \int_{V_{1}} \underline{G}_{o}^{A}\left(\vec{r}, \vec{r}^{\prime}\right) \cdot \vec{w}_{q}\left(\vec{r}^{\prime}\right) d v^{\prime} \\
\vec{B}_{q}(\vec{r}) & \equiv \int_{V_{1}} \nabla \times \underline{G}_{o}^{A}\left(\vec{r}, \vec{r}^{\prime}\right) \cdot \vec{w}_{q}\left(\vec{r}^{\prime}\right) d v^{\prime}
\end{aligned}
$$




\subsection{Generalized admittance matrix in the form of pole ex- pansions}

So far, we have obtained a representation of the generalized admittance matrix (see eq. (3.41)) that is not expressed as a pole expansion in the $s$-plane. In fact, that expression involves the inverted matrix $\left(\mathbf{A}+s^{2} \mathbf{B}\right)^{-1}$ which is frequency dependent.

In [60] it is presented a procedure that allow to generate reduced-order models in the $s$-domain from the integral form of Maxwell's equations, combining the Adaptive LanzcosPadè sweep (APLS), which is an improved form of the Padè via Lanzcos (PVL) algorithm, with the boundary element method. By using the mixed-potential integral equation, approximating the unknown current with Rao-Wilton-Glisson basis functions and applying the Galerkin's method, the authors obtain the following generalized matrix eigenvalue problem:

$$
\left[\mathbf{K}+s^{2} \mathbf{M}\right] J=s E
$$

where $\mathbf{k}$ and $\mathbf{M}$ are matrices that depend on the frequency through the Green's functions, $J$ is the unknown current and $E$ is the incident field. Thus, assuming that the matrices do not depend on the frequency and making a change of variables $b=s E$ and $u=s^{2}$, they rewrite the expression as:

$$
[\mathbf{K}+u \mathbf{M}] J=b
$$

In this way, they suggest to apply the ALPS procedure to find a piecewise rational-function approximation to the frequency response over the band of interest.

In our case, we have obtained a similar expression (see eq.(3.38)), which is even better, since matrices $\mathbf{A}$ and $\mathbf{B}$ are frequency independent. Therefore, we could apply at this point the ALPS algorithm in order to obtain a reduced order model of our structure, and thus a representation of the GAM in the form of pole expansion in the $s$-domain. Nevertheless we will follow a different and simpler procedure described in [73] to obtain it.

As we have seen in the previous section, the matrices $\mathbf{A}$ and $\mathbf{B}$ in the eigenvalue problem defined by (3.42) are positive definite. It is well known [105] that the matrix of eigenvectors $\mathbf{X}=\left(\mathbf{x}^{(1)}, \mathbf{x}^{(2)}, \ldots, \mathbf{x}^{(Q+M)}\right)$, permits the diagonalization of $\mathbf{A}$ and $\mathbf{B}$ :

$$
\mathbf{X}^{T} \mathbf{A X}=\Lambda=\operatorname{diag}\left\{k_{1}^{2}, k_{2}^{2}, \ldots, k_{Q+M}^{2}\right\}, \quad \mathbf{X}^{T} \mathbf{B X}=\mathbf{I}
$$

where I is the identity matrix. Using this expressions we can verify that:

$$
\left(\mathbf{A}+s^{2} \mathbf{B}\right)^{-1}=\mathbf{X}\left(\Lambda+s^{2} \mathbf{I}\right)^{-1} \mathbf{X}^{T}=\sum_{i=1}^{Q+M} \frac{\mathbf{x}^{(i)} \mathbf{x}_{T}^{(i)}}{k_{i}^{2}+s^{2}}
$$

By substituting this expression in the equation (3.41), we obtain the pole expansion of the generalized admittance matrix in the $s$-domain. At this point, we could also use different techniques [60] to obtain a reduced order model, i.e., to obtain the expression of the GAM with a reduced number of poles. However, we will follow an approach analogous to the 
approximations used in chapter 2 to find the mode expansion of the dyadic Green's functions. This is, we extract the static contribution of the inverted matrix in 'closed' form and then we find the frequency-dependent part as a pole expansion in the $s$-domain. The static contribution is

$$
\left.\left(\mathbf{A}+s^{2} \mathbf{B}\right)^{-1}\right|_{s=0}=\mathbf{A}^{-1}=\sum_{i=1}^{Q+M} \frac{\mathbf{x}^{(i)} \mathbf{x}_{T}^{(i)}}{k_{i}^{2}}
$$

Thus, we can write:

$$
\left(\mathbf{A}+s^{2} \mathbf{B}\right)^{-1}=\mathbf{A}^{-1}-s^{2} \sum_{i=1}^{Q+M} \frac{\mathbf{x}^{(i)} \mathbf{x}_{T}^{(i)}}{k_{i}^{2}\left(k_{i}^{2}+s^{2}\right)} \approx \mathbf{A}^{-1}-s^{2} \sum_{i=1}^{P} \frac{\mathbf{x}^{(i)} \mathbf{x}_{T}^{(i)}}{k_{i}^{2}\left(k_{i}^{2}+s^{2}\right)}
$$

It can be observed that after this extraction, the magnitude of the terms in the summation is reduced by the factor $s^{2} / k_{i}^{2}$. This fact allows to introduce the last approximation which consists in retaining the first $P$ terms with $k_{i} \leqslant \xi k_{\max }$, as in the case of the dyadic Green's function ( $\xi$ is an accuracy factor, usually $\xi=2,3$ ). On the other hand, it is noted that the calculation of $A^{-1}$ does note require any extra computational effort, since it is computed when solving the eigenvalue problem defined by (3.42).

Finally, introducing (3.49) in (3.41), we obtain the GAM in pole expansion form:

$$
\mathbf{Y}=\frac{1}{s \eta} \mathbf{Y}^{A}+\frac{s}{\eta} \mathbf{Y}^{B}-\frac{s^{3}}{\eta} \sum_{i} \frac{\mathbf{y}^{(i)} \mathbf{y}_{T}^{(i)}}{s^{2}+k_{i}^{2}}
$$

where

$$
\mathbf{Y}^{A}=\mathbf{G}, \quad \mathbf{Y}^{B}=\mathbf{T}-\mathbf{F}^{T} \mathbf{K}^{-2} \mathbf{F}+\mathbf{U}^{T} \mathbf{A}^{-1} \mathbf{U}, \quad \mathbf{y}^{(i)}=\frac{\mathbf{U}^{T} \mathbf{x}^{(\mathbf{i})}}{k_{i}}
$$

It is also remarkable that this expression fulfills all the requirements for the physical realizability of the admittance matrix of a lossless and reciprocal circuit:

- it is symmetrical,

- it is imaginary,

- it is an odd function of the frequency,

- the non-zero poles in the $k$-plane are present in real opposite pairs and have real residues,

- in self admittances, the residues of all poles are positive. 


\section{Chapter 4}

\section{Application of the State-Space Integral-Equation Formulation to the analysis of a rectangular cavity loaded with a dielectric cylinder}

\subsection{Introduction}

In the previous chapters we have presented a new integral-equation formulation in the $s$ domain to efficiently analyse the electromagnetic behaviour of perfect metallic cavities loaded with dielectric resonators. The linear, homogeneous and isotropic dielectric body is rigorously characterized by means of the electric equivalent polarization charge and current densities defined in the volume of the dielectric object. We express the electromagnetic field using the Green's functions of a cavity resonator instead of using the free-space ones as it is usually done in standard boundary integral methods. This way, by using the Green's functions for the scalar and vector potentials in the Coulomb's gauge, we obtain an hybrid representation of the field in terms of Green's integrals and rapidly converging modal series. Like in the boundary integral-resonant mode expansion method, taking as starting point this hybrid representation of the field and using the method of moments, we can obtain the modal chart and the field distribution of the cavity resonator by solving a real matrix linear eigenvalue problem. Moreover, the generalized admittance matrix of the structure is obtained in the form of pole expansions in the $s$-domain.

In this chapter, we will focus our attention on one of the main objectives of this $\mathrm{PhD}$ thesis: the application of this approach to characterize the electromagnetic response of a rectangular cavity loaded with a cylindrical dielectric body (see Fig. 4.1). In this case, we will use the Green's functions of a rectangular cavity. Note that in the formulation presented so far, we have always assumed that the cavity resonator used to calculate the Green's functions has the same geometry than the real metallic enclosure under analysis. This way, the boundary conditions over the metallic cavities are directly imposed by the Green's functions 
and there is no need to mesh the metallic shell. If we had used the Green's functions of a cavity resonator different from those of the structure under analysis, we should have taken into account the additional unknown current flowing through the walls of the real metallic cavity (see $[62,63,73]$ ). This would be the case, for instance, if we would like to analyse rectangular cavities with rounded corners loaded with dielectric resonators. However, this is beyond the scope of this $\mathrm{PhD}$ thesis, so we will focus our attention in the analysis of dielectric-loaded rectangular cavities.

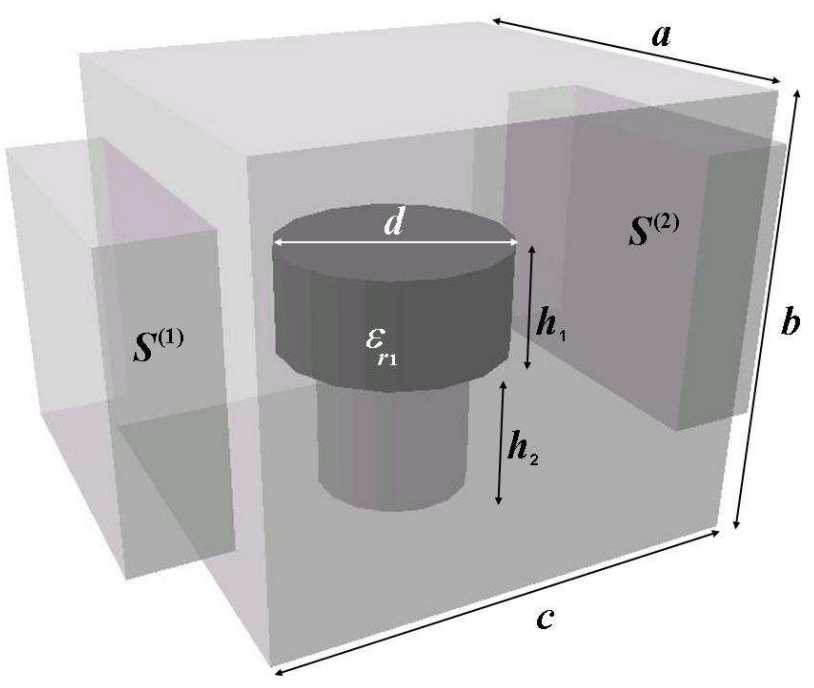

Figure 4.1: The basic building block under study: a cylindrical dielectric resonator arbitrarily placed inside a rectangular cavity connected to two rectangular waveguides.

In the second section of this chapter we describe the basis functions used to approximate the polarization vector that characterize the dielectric object inside the cavity. Throughout this chapter we show how crucial is the selection of an appropriate set of basis functions, not only for the good convergence of the method proposed, but also to transform some of the integrals involved in the problem. The numerical efficiency of the proposed method depends critically on the computation of the matrices collected in Table 3.1, and specially of those matrices involving the Green's functions. In the third section we explain how we have calculated the entries of these matrices.

The elements of the $\mathbf{S}$ and $\mathbf{D}$ matrices can be expressed in closed form, and can be easily reduced to 1- or 2-dimension integrals (some of them even have analytical solutions).

As it was presented in the work of San Blas [75], analytical solutions can be found to calculate the $\mathbf{F}, \mathbf{T}$ and $\mathbf{G}$ matrix elements. For this purpose, the access ports of the dielectric loaded resonator are assumed to be rectangular waveguides with dimensions equal to those of the corresponding cavity side. Furthermore, following a similar procedure to the used in that work, the $\mathbf{W}$ matrix elements can be efficiently computed once the integrals involved are reduced to 3-dimension integrals.

In the evaluation of the $\mathbf{C}$ and $\mathbf{L}$ matrix entries, a singular behavior arises when the 
source and the observation points coalesce $\left(\vec{r}=\vec{r}^{\prime}\right)$, and therefore their computation must be treated carefully. In order to overcome this inconvenience, the inherent singularity of the involved Green's functions has been analytically extracted (see section 2.6). Thus, the integrals present in both matrices have been split into two parts: the first one involving the singularity of the Green's function, and the other one including the regular terms of the Green's functions $[73,74]$. To evaluate the regular integrals, the scalar and dyadic Green's functions have been efficiently computed by means of the Ewald's technique, as described in [101].

In order to compute the singular part of the $\mathbf{C}$ matrix elements, all the surface-surface integrals have been reduced to three-dimension integrals. For the singular integrals related with the top and bottom bases of the dielectric cylinder, the formula $1 / R=-\nabla_{s} \cdot \nabla_{s} R$ allows to transform them into regular ones [106]. However, such formula can not be applied to the double surface integrals evaluated on the lateral surface of the cylinder. Nevertheless, an analytical and efficient extraction of the singularity has been made in such a case, allowing to transform the singular integrals into regular ones.

Finally, special attention must be paid to the calculation of the $\mathbf{L}$ matrix elements. In this case, it is crucial not only to transform the singularities of the integrals to regular expressions, but also to reformulate the volume-volume integrals to surface-surface ones. Thus, we only need to perform a 2-D mesh over the surface of the dielectric resonator, instead of performing a 3-D mesh in its volume. These transformations are specially important for the evaluation of the regular part of the $\mathbf{L}$ matrix, since they allow to drastically reduce the required computational effort.

\subsection{Basis functions}

In section 3.3 we have cast the integral equation (3.31a) into a linear matrix eigenvalue problem by means of the Method of Moments. For this purpose, we have used equation (3.32) to express the polarization vector that characterize the dielectric body in terms of a set of basis functions. We recall that these basis functions are defined inside the dielectric resonator (within $V_{1}$ ), where they are continuous and solenoidal. As it has been mentioned before, the selection of a suitable set of basis functions is a key issue in order to implement an efficient algorithm, since the convergence of the method depends critically on it.

Rigorous analysis of dielectric resonators (DR) is a complex task. The mathematical description of the electromagnetic field in such a structure is much more complicated than the field description in a hollow waveguide, since the number of operations and unknowns involved in the problem is much larger. Due to this fact, the application of mathematical models that approach the solution of the electromagnetic field in the DR in a simple way is of great practical interest for design purposes. These kind of simple models lack of the needed accuracy for modern circuit design, but they can give us a helping hand to better understand the DR electromagnetic behaviour and, thus, to choose a proper set of basis 
functions to express the polarization vector that characterise the dielectric object.

It is well known that an approximate solution for a dielectric resonator with high relative permittivity can be found by assuming that all the surfaces of the DR are perfect magnetic conductors [19]. Such a "first-order" model is shown in Fig. 4.2(a) where a cylindrical DR with diameter $d$ and height $h$ is depicted. It simply consists of a cylindrical cavity resonator with perfect magnetic walls. Using known procedures for the analysis of cavity resonators, it is easy to compute the resonant frequencies of this model. Unfortunately, this first-order model yields to high errors (10-20\%) when comparing the resonant frequencies computed with measurements. This fact evidences the needed of more complex models that provide more accurate results.

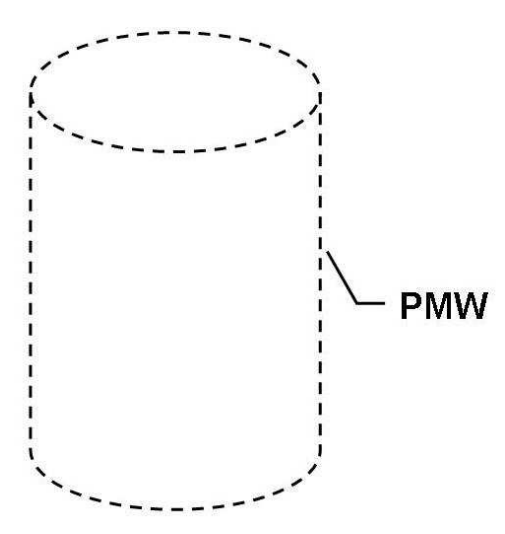

(a) First-order model

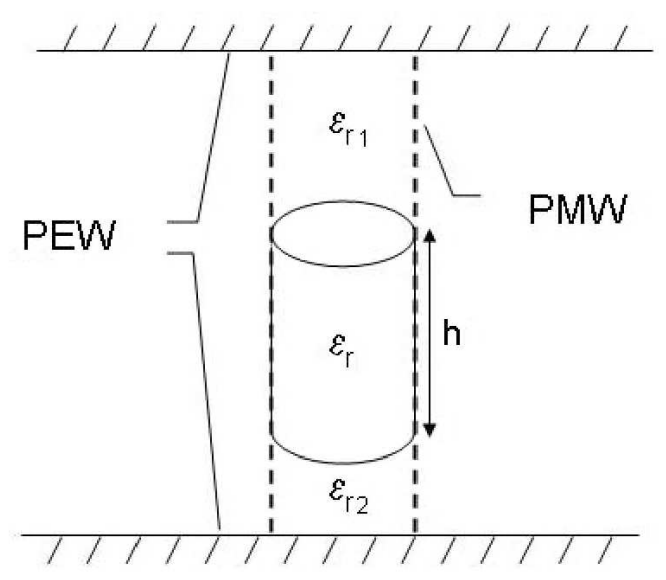

(b) Second-order model: Modified Cohn model

Figure 4.2: Simple models to analyse DR

An improved model was presented in 1968 by Seymour B. Cohn [14]. This approach is based on a second-order model described by Okaya and Barash [9] for the computation of resonant frequencies of $T E$ and $T M$ modes in rectangular dielectric resonators, which was later applied to cylindrical shapes $[10,11]$. In this model, the cylindrical perfect magnetic conductor shell is retained, but the PMW at the top and bottom of the DR are replaced by air-filled waveguides. These two hollow waveguides operate below cutoff because they are filled with a low dielectric constant. Thus, the modes in these hollow waveguides are evanescent and decay exponentially in the direction away from each end of the DR. The equivalent circuit is a propagating transmission line of length $h$ terminated at each end by reactances equal to the pure-imaginary characteristic impedance of the cutoff air-filled waveguide. Using this second-order model, Cohn obtained not only the resonant frequencies of the DR, but also solutions for the coupling between adjacent dielectric resonators operating at the fundamental mode.

The Cohn model is appropriate to characterise an isolated DR, however we are interested in analysing a DR enclosed within a rectangular metal shield. For this purpose, the modified Cohn model can be used. It consists of placing the parallel plate metal enclosure as shown in 
Fig.4.2(b). In this case, the waveguides at the top and bottom of the DR may have different dielectric constants. Both the $T E_{01 \delta}$ and $H E_{11}$ modes of a DR can be solved by means of this technique. Further improvements can be obtained by using a perturbational correction of this model as reported in [19], or making use of variational techniques [25] to better approximate the $T E_{01 \delta}^{o}$ mode. Another simple model was described in the 70's by Itoh and Rudokas [24], who instead of using the PMW waveguide, considered a dielectric rod waveguide.
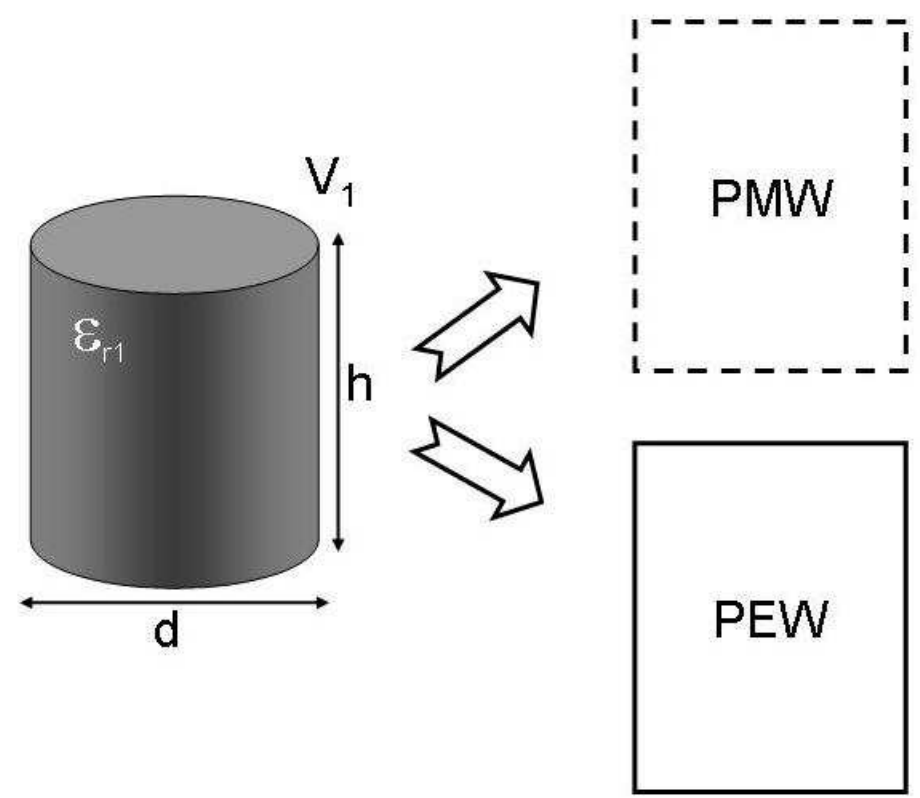

Figure 4.3: Diagram of the basis functions used in the polarization vector expansion: The solenoidal eigenvectors of the cylindrical cavity (with diameter $d$ and height $h$ ) containing the DR are used both with perfect electric walls (PEW) and perfect magnetic walls (PMW).

In our case, the basis functions needed by the Method of Moments are defined inside the dielectric resonator. As it has been mentioned in section 3.2, due to the fact that the dielectric material under analysis is linear, isotropic and homogeneous, the induced polarization is a solenoidal function for the points within the volume $V_{1}$. Furthermore, from the simple models considered above, we know that a first-order solution for a dielectric resonator with high relative permittivity can be found by considering it as a cavity enclosed by perfect magnetic walls. As a consequence, a good initial approach consists on expanding the polarization vector unknown in terms of the set of solenoidal magnetic eigenvectors of the cylindrical cavity containing the DR. However, this is a first-order approach that yields to inaccurate results [19]. In fact, it can be easily observed that, since the solenoidal magnetic eigenvectors have no normal component on the cylinder surface $\left(S_{1}\right)$, the induced surface charge density described by equation (3.17) can not be expressed in terms of this set of basis functions. For this reason, the solenoidal electric eigenvectors (obtained as the solution of the equivalent cylindrical cavity with perfect electric walls) must also be included in the complete set of 
solenoidal basis functions.

Therefore, the set of basis functions used in the present formulation can be split into two different subsets: the solenoidal electric eigenvectors of the cylindrical cavity, solution of the perfect electric wall cavity, and the solenoidal magnetic ones, solution of the perfect magnetic wall cavity [62] (see Table 4.1). In Figs. 4.4 and 4.5 some examples of basis functions are shown, for the case in which the dielectric puck has a ratio $d / h=2.9$.

The basis functions are defined in the cylindrical coordinated system $\left(\widehat{u}_{\rho}, \widehat{u}_{\varphi}, \widehat{u}_{z}\right)$ located in the center of the bottom cap of the cylindrical cavity. Each subset of basis functions are made up of $T E^{z}$ and $T M^{z}$ modes. We note that these are full-domain basis functions and they satisfy the following equations:

$$
\nabla \cdot \vec{w}_{q}=0, \quad \nabla \times \nabla \times \vec{w}_{q}=-\nabla^{2} \vec{w}_{q}=k_{q}^{2} \vec{w}_{q}, \quad \text { in } V_{1}
$$

where $k_{q}$ is the resonant wavenumber related to the $q$-th basis function $\vec{w}_{q}$. The properties expressed in equation (4.1) are crucial, as it will be shown in the next section, for the analytical transformation of the integrals involved in the calculation of the matrices defined in Table 3.1, specially for the case of the $\mathbf{L}$ matrix.

The basis functions are normalized:

$$
\int_{V_{1}} \vec{w}_{i} \cdot \vec{w}_{j} d v=\delta_{i j}
$$

where both basis functions, $\vec{w}_{i}$ and $\vec{w}_{j}$, are solution of the cylindrical cavity with perfect electric walls (PEW) or both are solution of the cylindrical cavity with perfect magnetic walls (PMW).

Finally, it is remarkable that we may follow different criteria to sort the basis functions (from lower to higher resonant wavenumbers, from lower to higher modal indexes, etc.). In chapter 5 we will show how the election among one of these criteria may affect the convergence of the algorithm developed. 
Table 4.1: Basis Functions Used in the Method of Moments

The normalized basis functions are defined in the cylindrical coordinated system $\left(\widehat{u}_{\rho}, \widehat{u}_{\varphi}, \widehat{u}_{z}\right)$ located in the center of the dielectric puck base (see Fig. (4.6)). The indexes $j \equiv(p, q, l, m)$ with $(m) \equiv($ even $),(o d d) \equiv(1),(2)$ are used, and the operator $\nabla_{t}$ is the projection of the $\nabla$ operator in the plane defined by $\left(\widehat{u}_{\rho}, \widehat{u}_{\varphi}\right)$.

\section{Perfect Electric Wall}

$T E^{z}: \vec{w}_{j}=\frac{\sqrt{2 / h}}{\Theta_{p q}^{\prime}}\left(\widehat{u}_{z} \times \nabla_{t} \psi_{p q}^{(m)}\right) \sin \left(\beta_{l} z\right)$

$T M^{z}: \vec{w}_{j}=\frac{\sqrt{\epsilon_{l} / h}}{\Theta_{p q} \sqrt{\Theta_{p q}^{2}+\beta_{l}^{2}}}\left[\beta_{l} \sin \left(\beta_{l} z\right) \nabla_{t} \phi_{p q}^{(m)}-\Theta_{p q}^{2} \phi_{p q}^{(m)} \cos \left(\beta_{l} z\right) \widehat{u}_{z}\right]$

Perfect Magnetic Wall

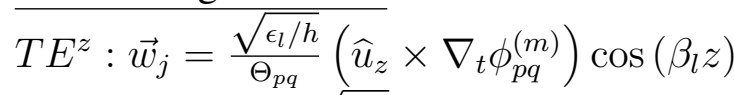

$T M^{z}: \vec{w}_{j}=\frac{-\sqrt{2 / h}}{\Theta_{p q}^{\prime} \sqrt{\Theta_{p q}^{\prime 2}+\beta_{l}^{2}}}\left[\beta_{l} \cos \left(\beta_{l} z\right) \nabla_{t} \psi_{p q}^{(m)}+\Theta_{p q}^{\prime 2} \psi_{p q}^{(m)} \sin \left(\beta_{l} z\right) \widehat{u}_{z}\right]$

SYMBOLS: $J_{p}(x)$ is the Bessel function of the first kind and the order $\mathrm{p} ; J_{p}^{\prime}(x)$ is its derivative; $\chi_{p q}$ and $\chi_{p q}^{\prime}$ are the q-th non-zero root of: $J_{p}(x)=0$ and $J_{p}^{\prime}(x)=0$, respectively; $\Theta_{p q} \equiv 2 \chi_{p q} / d ; \Theta_{p q}^{\prime} \equiv 2 \chi_{p q}^{\prime} / d ; \beta_{l} \equiv l \pi / h ; \epsilon_{p}$ is the Neumman Factor; the scalar functions are:

$$
\begin{aligned}
& \psi_{p q}^{(m)}=N_{p q} J_{p}\left(\Theta_{p q}^{\prime} \rho\right)\left\{\begin{array}{l}
\cos (p \varphi) \\
\sin (p \varphi)
\end{array}\right\} \begin{array}{l}
(m)=(1) \\
(m)=(2)
\end{array} ; \quad N_{p q}=\frac{\Theta_{p q}^{\prime}}{J_{p}\left(\chi_{p q}^{\prime}\right)} \sqrt{\frac{\epsilon_{p} / \pi}{\chi_{p q}^{\prime 2}-p^{2}}} \\
& \phi_{p q}^{(m)}=M_{p q} J_{p}\left(\Theta_{p q} \rho\right)\left\{\begin{array}{l}
\cos (p \varphi) \\
\sin (p \varphi)
\end{array}\right\} \begin{array}{l}
(m)=(1) \\
(m)=(2)
\end{array} ; \quad M_{p q}=\frac{2 \sqrt{\epsilon_{p} / \pi}}{d J_{p+1}\left(\chi_{p q}\right)}
\end{aligned}
$$




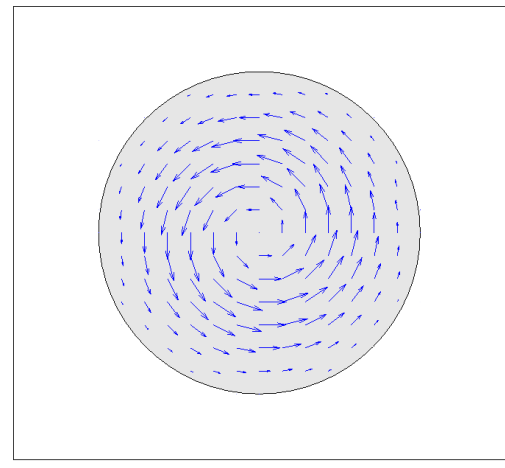

(a) PEWTE $E^{z}:(0,1,1)$; (e)

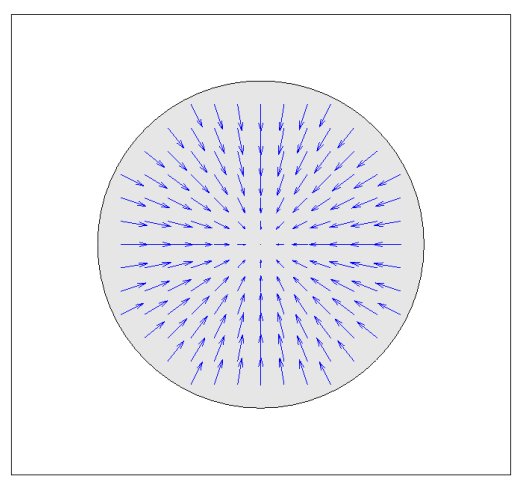

(c) $P E W T M^{z}:(0,1,1)$; (e)

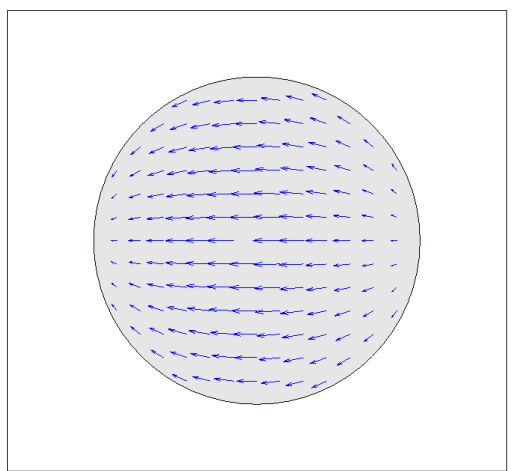

(e) $P M W T M^{z}:(1,1,1) ;(e)$

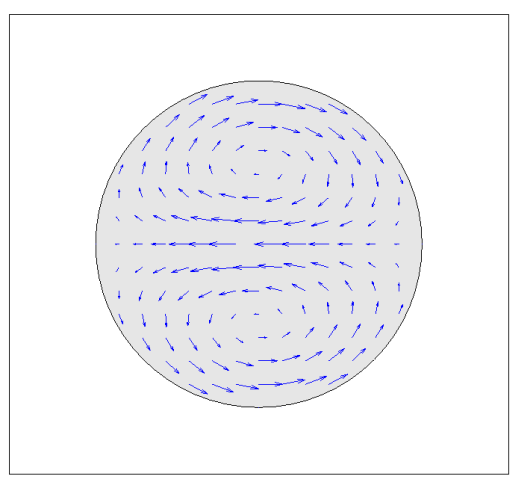

(g) $P M W T E^{z}:(1,1,0) ;(o)$

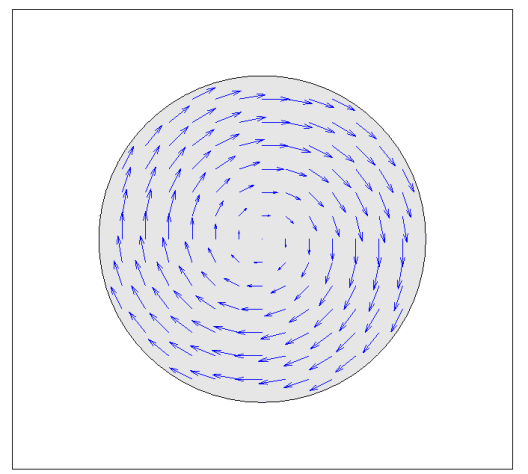

(b) $P M W T E^{z}:(0,1,0)$; (e)

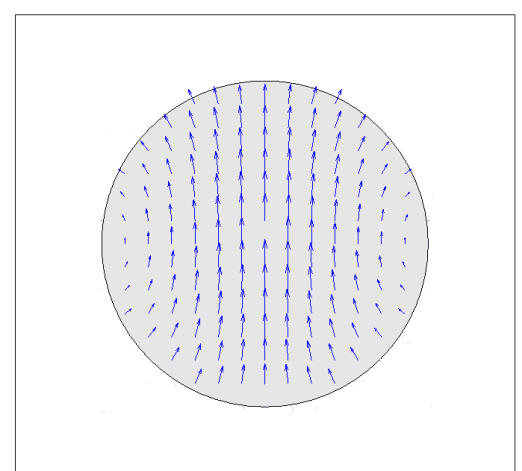

(d) PEWTE $E^{z}:(1,1,1)$; (e)

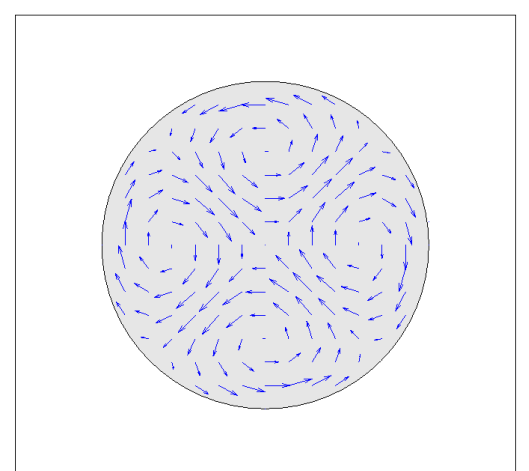

(f) $P M W T E^{z}:(2,1,0) ;(e)$

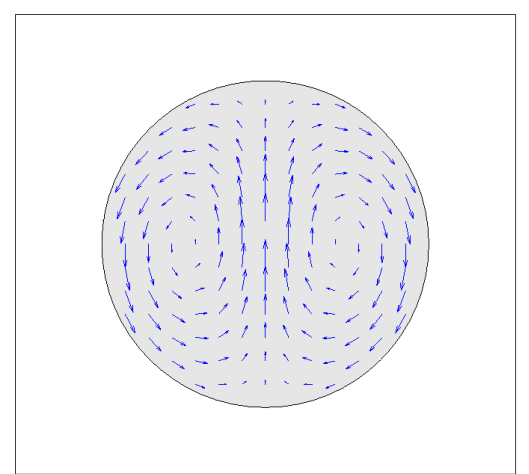

(h) $P M W T E^{z}:(1,1,0) ;(e)$

Figure 4.4: Basis functions used by the MoM in equatorial plane of the DR. 


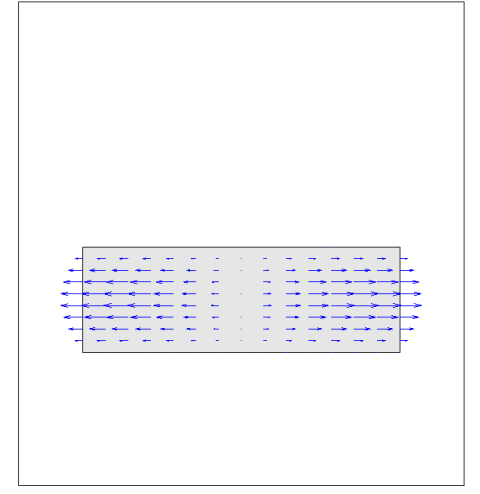

(a) PEWTE $E^{z}:(2,1,1),(o)$

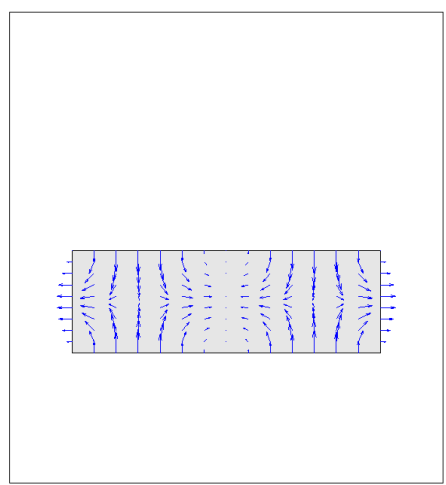

(c) $P E W T M^{z}:(2,1,1) ;(e)$

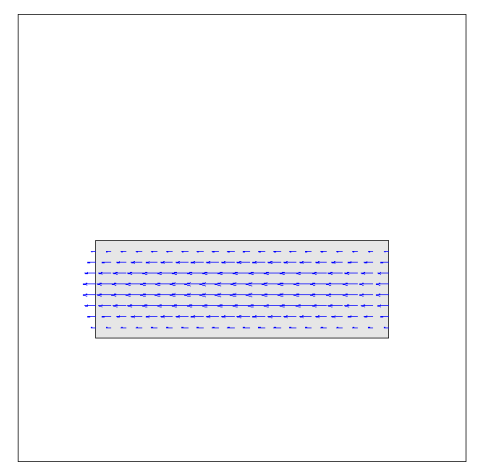

(e) $P E W T E^{z}:(1,1,1) ;(o)$

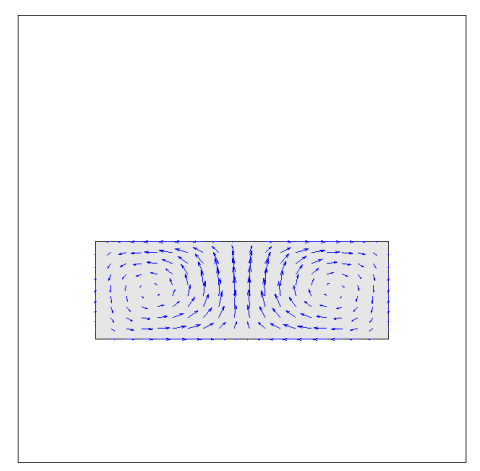

(g) $P M W T M^{z}:(0,1,1) ;(e)$

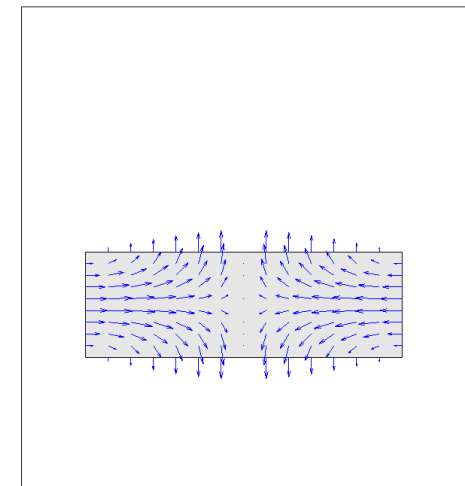

(b) PEWTM $M^{z}:(0,1,1),(e)$

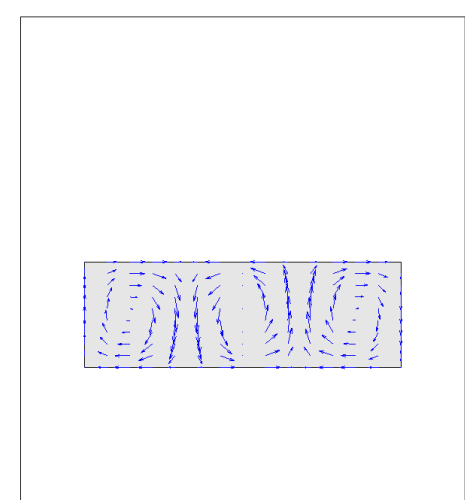

(d) $P M W T M^{z}:(1,2,1) ;(o)$

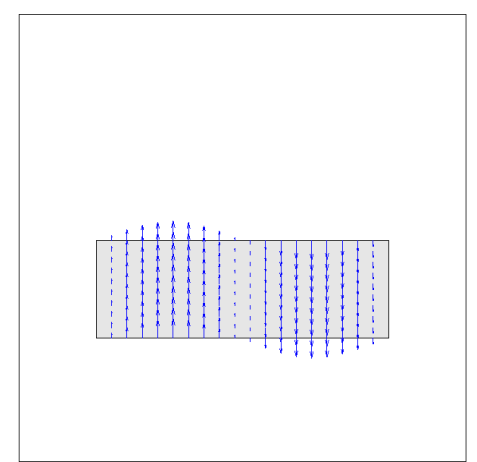

(f) PEWTM" : $(1,1,0)$; (e)

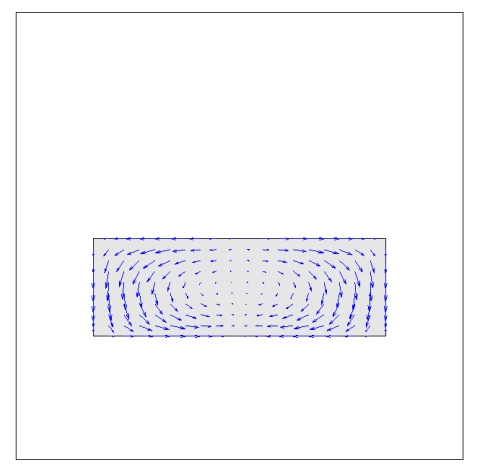

(h) PMWT $M^{z}:(2,1,0)$; $(e)$

Figure 4.5: Basis functions used by the MoM in meridian plane of the DR. Figs. 4.5(a)4.5(d) show the plane with $\phi=0$, whereas Figs. 4.5(e)-4.5(h) show the plane with $\phi=\pi / 2$. 


\subsection{Calculation of the matrices used in the MoM}

In the previous section we have described the set of basis functions used to expand the polarization vector to solve the electric field integral equation by means of the method of moments. The proper election of these basis functions is key to ensure a good convergence of the formulation proposed. However, the efficiency of the formulation implemented does not only depend on the basis functions selected, but also on the computation of the matrices collected in the Table 3.1, and specially of those matrices involving the Green's functions. In this section, the procedure followed to calculate each of these matrices is described.

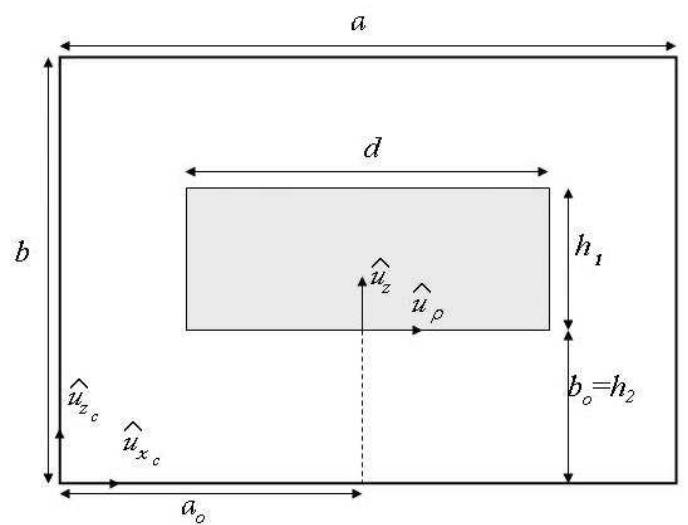

(a) Cross-section

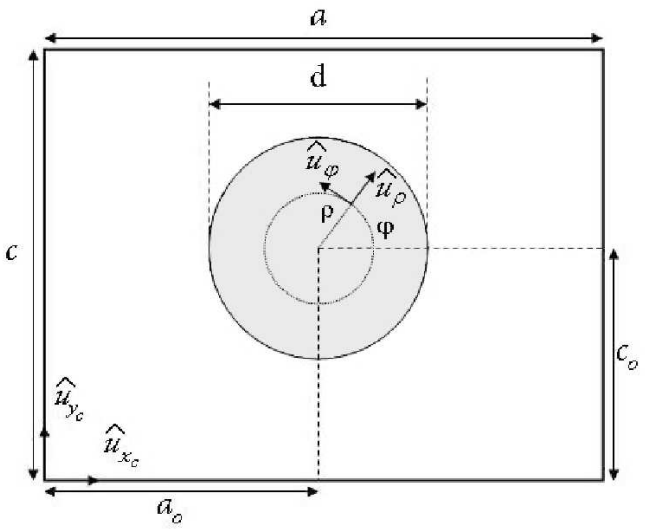

(b) Floor plan

Figure 4.6: Cartesian and polar coordinate systems associated to the rectangular cavity and to the cylindrical DR, respectively.

\subsubsection{Computation of the D matrix}

The $\mathbf{D}$ matrix is a real symmetric matrix whose entries are:

$$
D_{i j}=\int_{V_{1}} \vec{w}_{i}(\vec{r}) \cdot \vec{w}_{j}(\vec{r}) d v
$$

where $V_{1}$ is the cylindrical volume defined by the $\mathrm{DR}, \vec{w}_{i}(\vec{r})$ and $\vec{w}_{j}(\vec{r})$ are basis functions and $i, j=1,2, \ldots, Q$. We recall that $Q$ is the number of basis functions used to expand the polarization vector by the method of moments in (3.32).

To calculate this matrix, we have to take into account the following possible combinations of basis functions:

- PEW-PEW: both of the basis functions are solution of the cylindrical cavity with perfect electric walls

- PMW-PMW: both of the basis functions are are solution of of the cylindrical cavity with perfect magnetic walls 
- PEW-PMW: one of the basis functions is solution of the PEW cavity whereas the other one is eigenvector of the PMW cavity

\section{A. Case PEW-PEW and PMW-PMW}

Since the basis functions used in the method of moments are the solenoidal modes of the cylindrical cavity with perfect electric and magnetic walls, they must satisfy, respectively, the properties of the electric and magnetic normalized eigenvectors of a cavity resonator described in section 2.2. They must verify (4.2), and therefore, the value of the matrix $\mathbf{D}$ in the PEW-PEW and PMW-PMW cases, is:

$$
D_{i j}=\delta_{i j}
$$

\section{B. Case PEW-PMW}

Let assume that the function $\vec{w}_{i}(\vec{r})$ is a solenoidal mode of the cylindrical cavity with perfect electric walls, and the function $\vec{w}_{j}(\vec{r})$ is a solenoidal mode of the cavity with perfect magnetic walls. By using the notation used in Table 4.1, we can introduce the expressions of the basis functions. The indexes of each basis function are related to the modal indexes $(i) \Rightarrow(p, q, l, m) ;(j) \Rightarrow(r, s, t, n)$, where the indexes $(m),(n) \equiv(1),(2)$ indicates if the mode used is the even or the odd solution. Thus, we have to take into consideration now the different possible combinations of $T E^{z}$ and $T M^{z}$ modes. The integrals of the $\mathbf{D}$ matrix are calculated in the cylindrical coordinate system used to define the basis functions $\{\vec{w}\}$, which is placed in the center of the bottom cap of the dielectric puck $\left(\widehat{u}_{\rho}, \widehat{u}_{\varphi}, \widehat{u}_{z}\right)$ located in the center of the dielectric puck base (see Fig. (4.6)).

Case $\vec{w}_{i}$ is a $T E^{z}$ mode and $\vec{w}_{j}$ is a $T E^{z}$ mode:

By introducing the expressions of the $T E^{z}$ basis functions in equation (4.3), we can write:

$$
\begin{aligned}
D_{i j} & =\mathcal{N}_{i} \mathcal{N}_{j} \int_{V_{1}}\left(\widehat{u}_{z} \times \nabla_{s} \psi_{p q}^{(m)}\right) \cdot\left(\widehat{u}_{z} \times \nabla_{s} \phi_{r s}^{(n)}\right) \sin \left(\beta_{l} z\right) \cos \left(\beta_{t} z\right) d v \\
& =\mathcal{N}_{i} \mathcal{N}_{j} \int_{0}^{h} \sin \left(\beta_{l} z\right) \cos \left(\beta_{t} z\right) d z \int_{S_{C_{1}}} \nabla_{s} \psi_{p q}^{(m)} \cdot \nabla_{s} \phi_{r s}^{(n)} d s
\end{aligned}
$$

where $\nabla_{s}$ is the projection of the $\nabla$ operator in the plane defined by $\left(\widehat{u}_{\rho}, \widehat{u}_{\varphi}\right) ; \beta_{i} \equiv \frac{i \pi}{h} ; \psi_{p q}^{(m)}$ and $\phi_{r s}^{(n)}$ are the scalar functions defined in Table 4.1 and $S_{C_{1}}$ is the surface defined by the bottom cap of the cylinder ( $\mathrm{z}=0$ plane). To calculate the integration over the surface $S_{C_{1}}$, we use the following surface vector identity in cylindrical coordinates [85],

$$
\int_{S_{C_{1}}} \nabla_{s} \phi_{r s}^{(n)} \cdot \nabla_{s} \psi_{p q}^{(m)} d s=\int_{C_{1}} \phi_{r s}^{(n)}\left(\widehat{u}_{\rho} \cdot \nabla_{s} \psi_{p q}^{(m)}\right) d l-\int_{S_{C_{1}}} \phi_{r s}^{(n)} \nabla_{s}^{2} \psi_{p q}^{(m)} d s
$$

where $C_{1}$ is the contour that encloses the surface $S_{C_{1}}$. This way, equation (4.5) can be 
rewritten as follows:

$$
\begin{aligned}
D_{i j} & =\mathcal{N}_{i} \mathcal{N}_{j} \int_{0}^{h} \sin \left(\beta_{l} z\right) \cos \left(\beta_{t} z\right) d z\left[\oint_{\rho=\frac{d}{2}} \phi_{r s}^{(n)} \frac{\partial \psi_{p q}^{(m)}}{\partial \rho} \rho d \varphi+\int_{S_{C_{1}}} \phi_{r s}^{(n)} \nabla_{s}^{2} \psi_{p q}^{(m)} d s\right] \\
& =\mathcal{N}_{i} \mathcal{N}_{j} \Theta_{p q}^{\prime 2} \int_{0}^{h} \sin \left(\beta_{l} z\right) \cos \left(\beta_{t} z\right) d z \int_{S_{C_{1}}} \psi_{p q}^{(m)} \phi_{r s}^{(n)} d s
\end{aligned}
$$

where we have taken into account that

$$
\left.\frac{\partial \psi_{p q}^{(m)}}{\partial \rho}\right|_{\rho=\frac{d}{2}} ; \quad \nabla_{s}^{2} \psi_{p q}^{(m)}=-\Theta_{p q}^{\prime 2} \psi_{p q}^{(m)}
$$

We can now perform the analytical integration over the $z$ and $\varphi$ variables, to obtain the value of the $\mathbf{D}$ matrix entry:

$$
\begin{array}{cl}
D_{i j}=\mathcal{N}_{i} \mathcal{N}_{j} \Theta_{p q}^{\prime 2} \frac{2}{\epsilon_{p}} \frac{\left(1-(-1)^{l+t}\right) l}{l^{2}-t^{2}} h \delta_{p r} \delta_{m n} I_{p q, r s}^{\rho} & \forall l \neq t \\
D_{i j}=0 & \forall l=t
\end{array}
$$

where the integral $I_{p q, r s}^{\rho}$ is defined as:

$$
I_{p q, r s}^{\rho} \equiv \int_{0}^{\frac{d}{2}} \rho J_{p}\left(\Theta_{p q}^{\prime} \rho\right) J_{r}\left(\Theta_{r s} \rho\right) d \rho
$$

which is computed numerically.

Case $\vec{w}_{i}$ is a $T M^{z}$ mode and $\vec{w}_{j}$ is a $T M^{z}$ mode:

By substituting the expressions of $\vec{w}_{i}(\vec{r})$ and $\vec{w}_{j}(\vec{r})$ by its value when both of them are $T M^{z}$ modes in equation (4.3), we have:

$$
\begin{aligned}
D_{i j}=\mathcal{N}_{i} \mathcal{N}_{j} \int_{V_{1}}\left[\beta_{l} \sin \left(\beta_{l} z\right) \nabla_{s} \phi_{p q}^{(m)}-\Theta_{p q}^{2} \phi_{p q}^{(m)} \cos \left(\beta_{l} z\right) \widehat{u}_{z}\right] \\
{\left[-\beta_{t} \cos \left(\beta_{t} z\right) \nabla_{s} \psi_{r s}^{(n)}-\Theta_{r s}^{\prime 2} \psi_{r s}^{(n)} \sin \left(\beta_{t} z\right) \widehat{u}_{z}\right] d v } \\
=\mathcal{N}_{i} \mathcal{N}_{j}\left(I_{1}+I_{2}\right)
\end{aligned}
$$

where the integrals $I_{1}$ and $I_{2}$ are:

$$
\begin{aligned}
& I_{1} \equiv-\beta_{l} \beta_{t} \int_{V_{1}} \sin \left(\beta_{l} z\right) \cos \left(\beta_{t} z\right)\left(\nabla_{s} \phi_{p q}^{(m)}\right) \cdot\left(\nabla_{s} \psi_{r s}^{(n)}\right) d v \\
& I_{2} \equiv \Theta_{p q}^{2} \Theta_{r s}^{\prime 2} \int_{V_{1}} \cos \left(\beta_{l} z\right) \sin \left(\beta_{t} z\right) \phi_{p q}^{(m)} \psi_{r s}^{(n)} d v
\end{aligned}
$$


This way, using again the relation (4.6) to transform the integral $I_{1}$, and integrating both $I_{1}$ and $I_{2}$ over $z$ and $\varphi$, we obtain:

$$
\begin{array}{cl}
D_{i j}=\mathcal{N}_{i} \mathcal{N}_{j}\left(\beta_{l} \beta_{t} l+\Theta_{p q}^{2} t\right) \Theta_{r s}^{\prime 2} \frac{1-(-1)^{l+t}}{t^{2}-l^{2}} h \delta_{m n} I_{r s, p q}^{\rho} & \forall l \neq t \\
D_{i j}=0 & \forall l=t
\end{array}
$$

where $I_{r s, p q}^{\rho}$ is the integral defined in equation (4.9).

Case $\vec{w}_{i}$ is a $T E^{z}$ mode and $\vec{w}_{j}$ is a $T M^{z}$ mode:

When $\vec{w}_{i}(\vec{r})$ is a $T E^{z}$ mode of the PEW cavity and $\vec{w}_{j}(\vec{r})$ is a $T M^{z}$ eigenvector of the PMW cavity, then we have that the entry $i j$ of the $\mathbf{D}$ matrix is:

$$
\begin{aligned}
D_{i j} & =-\mathcal{N}_{i} \mathcal{N}_{j} \int_{V_{1}} \sin \left(\beta_{l} z\right)\left(\widehat{u}_{z} \times \nabla_{s} \psi_{p q}^{(m)}\right) \cdot\left[\beta_{t} \cos \left(\beta_{t} z\right) \nabla_{s} \psi_{r s}^{(n)}+\Theta_{r s}^{\prime 2} \sin \left(\beta_{t} z\right) \psi_{r s}^{(n)} \widehat{u}_{z}\right] d v \\
& =\mathcal{N}_{i} \mathcal{N}_{j} \beta_{t} \int_{0}^{h} \sin \left(\beta_{l} z\right) \cos \left(\beta_{t} z\right) d z \int_{S_{C_{1}}}\left(\widehat{u}_{z} \times \nabla_{s} \psi_{p q}^{(m)}\right) \cdot \nabla_{s} \psi_{r s}^{(n)} d s
\end{aligned}
$$

To further develop this expression, we can make use of the following surface vector identity in cylindrical coordinates:

$$
\int_{S_{C_{1}}} \vec{a} \cdot \nabla_{s} \psi d s=\int_{C_{1}} \psi \vec{a} \cdot \widehat{u}_{\rho} d l-\int_{S_{C_{1}}} \psi \nabla_{s} \cdot \vec{a} d s
$$

This way, we can rewrite equation (4.13) as ${ }^{8}$ :

$$
D_{i j}=\mathcal{N}_{i} \mathcal{N}_{j} \beta_{t} \frac{d}{2} \int_{0}^{h} \sin \left(\beta_{l} z\right) \cos \left(\beta_{t} z\right) d z \oint_{\rho=\frac{d}{2}} \psi_{r s}^{(n)} \widehat{u}_{\varphi} \cdot \nabla_{s} \psi_{p q}^{(m)} d \varphi
$$

Finally, the integration over $z$ and $\varphi$ can be analytically performed, obtaining:

$$
\begin{array}{cc}
D_{i j}=\mathcal{N}_{i} \mathcal{N}_{j} \beta_{t} J_{p}\left(\chi_{p q}^{\prime}\right) J_{r}\left(\chi_{r s}^{\prime}\right) h \frac{\left(1-(-1)^{l+t}\right) l}{l^{2}-t^{2}} p(-1)^{m}\left(1-\delta_{m n}\right) \delta_{p r} & \forall l \neq t \\
D_{i j}=0 & \forall l=t
\end{array}
$$

\footnotetext{
${ }^{8}$ To achieve this result, we have defined $\vec{a}=\widehat{u}_{z} \times \nabla_{s} \psi_{p q}^{(m)}$ in (4.14), and we have used the following identities:

$$
\nabla_{s} \cdot(\vec{a} \times \vec{b})=\vec{b} \cdot \nabla_{s} \times \vec{a}-\vec{a} \cdot \nabla_{s} \times \vec{b}, \quad \widehat{u}_{z} \cdot \nabla_{s} \times \nabla_{s} \psi=\nabla_{s} \cdot\left(\nabla_{s} \times\left(\psi \widehat{u}_{z}\right)\right)=0
$$
}


Case $\vec{w}_{i}$ is a $T M^{z}$ mode and $\vec{w}_{j}$ is a $T E^{z}$ mode:

In this last case, the element $i j$ of the $\mathbf{D}$ matrix is expressed by:

$$
\begin{aligned}
D_{i j} & =-\mathcal{N}_{i} \mathcal{N}_{j} \int_{V_{1}}\left[\beta_{l} \sin \left(\beta_{l} z\right) \nabla_{s} \phi_{p q}^{(m)}+\Theta_{p q}^{2} \cos \left(\beta_{l} z\right) \phi_{p q}^{(m)} \widehat{u}_{z}\right] \cdot\left[\cos \left(\beta_{t} z\right)\left(\widehat{u}_{z} \times \nabla_{s} \phi_{r s}^{(n)}\right)\right] d v \\
& =\mathcal{N}_{i} \mathcal{N}_{j} \beta_{l} \int_{0}^{h} \sin \left(\beta_{l} z\right) \cos \left(\beta_{t} z\right) d z \int_{S_{C_{1}}}\left(\widehat{u}_{z} \times \nabla_{s} \phi_{r s}^{(n)}\right) \cdot \nabla_{s} \phi_{p q}^{(m)} d s
\end{aligned}
$$

Following the same procedure as in the $T E^{z}-T M^{z}$ case, we have:

$$
D_{i j}=-\mathcal{N}_{i} \mathcal{N}_{j} \beta_{l} \frac{d}{2} \int_{0}^{h} \sin \left(\beta_{l} z\right) \cos \left(\beta_{t} z\right) d z \oint_{\rho=\frac{d}{2}} \phi_{p q}^{(m)} \widehat{u}_{\varphi} \cdot \nabla_{s} \phi_{r s}^{(n)} d \varphi
$$

Since $\left.\phi_{p q}^{(m)}\right|_{\rho=\frac{d}{2}}=0$, then

$$
D_{i j}=0
$$

\subsubsection{Computation of the $S$ matrix}

The entries of the $\mathbf{S}$ matrix are represented by:

$$
S_{i j}=\int_{V_{1}} \vec{E}_{i}(\vec{r}) \cdot \vec{w}_{j}(\vec{r}) d v
$$

where $\vec{E}_{i}(\vec{r})$ is a electric solenoidal mode of the empty rectangular cavity and $i=1,2, \ldots, M$, being $M$ the number of modes used in the expansion of the dyadic Green's function, whereas $\vec{w}_{j}(\vec{r})$ is a basis function and $j=1,2, \ldots, Q$, being $Q$ the number of basis functions used to expand the polarization vector by the method of moments.

To evaluate the expression (4.20), we use, once again, the cylindrical coordinate system $\left\{\widehat{u}_{\rho}, \widehat{u}_{\varphi}, \widehat{u}_{z}\right\}$ placed in the center of the bottom cap of the dielectric puck. Since the electric modes of the empty rectangular cavity have been calculated in the rectangular coordinate system $\left\{\widehat{u}_{x_{c}}, \widehat{u}_{y_{c}}, \widehat{u}_{z_{c}}\right\}$ placed in the corner of the cavity (see Appendix A), a transformation of coordinate system is required. As it can be observed in Fig. 4.6, the rectangular coordinate system can be expressed in the cylindrical one:

$$
\begin{aligned}
& x_{c}=\rho \cos \varphi+a_{o} \\
& y_{c}=\rho \sin \varphi+c_{o} \\
& z_{c}=z+b_{o}
\end{aligned}
$$

In order to evaluate the entries of the $\mathbf{S}$ matrix, we will first consider the division between $T E^{z_{c}}$ and $T M^{z_{c}}$ modes for both the basis functions and the electric solenoidal modes of the 
rectangular cavity. This way, after performing some previous calculations, we can take into account whether the basis functions belong to the PEW cylindrical cavity or to the PMW one. Furthermore, we will consider that $\left(i_{x_{c}}, i_{y_{c}}, i_{z_{c}}\right)$ represent the modal indexes associated to the $i$-th mode of the rectangular cavity $\vec{E}_{i}(\vec{r})$, related to the coordinates $\left(x_{c}, y_{c}, z_{c}\right)$, respectively; and that the indexes $(p, q, l)$ represent the modal indexes associated to the $j$-th basis function $\vec{w}_{j}(\vec{r})$.

\section{A. Case $\vec{E}_{i}$ is a $\mathbf{T} E^{\mathbf{z}_{\mathbf{c}}}$ mode and $\vec{w}_{j}$ is a $\mathbf{T} E^{\mathbf{z}_{\mathbf{c}}}$ basis function}

Since the $T E^{z_{c}}$ basis function $\vec{w}_{j}(\vec{r})$ may be the solenoidal eigenvector of the PEW cylindrical cavity or the solution of the PMW one, we define the following scalar functions to simplify the calculations:

$$
\Gamma_{l}(z) \equiv\left\{\begin{array}{c}
\sin \left(\beta_{l}^{h} z\right) \\
\cos \left(\beta_{l}^{h} z\right)
\end{array}\right\} ; \quad \Delta_{p q} \equiv\left\{\begin{array}{c}
\Theta_{p q}^{\prime} \\
\Theta_{p q}
\end{array}\right\} ; \quad \Lambda_{p q}^{(m)} \equiv\left\{\begin{array}{c}
\psi_{p q}^{(m)} \\
\phi_{p q}^{(m)}
\end{array}\right\} ; \quad \begin{aligned}
& \vec{w}_{j} \in P E W \\
& \vec{w}_{j} \in P M W
\end{aligned}
$$

where $\beta_{h}^{l} \equiv \frac{l \pi}{h}$ and the scalar functions $\Theta_{p q}^{\prime}, \Theta_{p q}, \psi_{p q}^{(m)}, \phi_{p q}^{(m)}$ are defined in Table 4.1.

This way, we can express a $T M^{z_{c}}$ basis function, independently whether it is solution of the PEW cylindrical cavity or solution of the PMW one, as:

$$
\vec{w}_{j}(\vec{r})=\mathcal{N}_{j}^{b f} \Gamma_{l}(z)\left(\widehat{u}_{z} \times \nabla_{s} \Lambda_{p q}^{(m)}\right)
$$

Using expression (4.22) and the $T E^{z_{c}}$ solenoidal mode of the empty rectangular cavity, described in (A.15), in equation (4.20), we can write:

$$
\begin{aligned}
S_{i j} & =\mathcal{N}_{i}^{r c} \mathcal{N}_{j}^{b f} \int_{0}^{h} \sin \left(k_{z_{c}, i}\left(z+b_{o}\right)\right) \Gamma_{l}(z) d z \int_{S_{C_{1}}}\left(\widehat{u}_{z} \times \nabla_{s} \xi_{i_{x_{c}} i_{y_{c}}}\right) \cdot\left(\widehat{u}_{z} \times \nabla_{s} \Lambda_{p q}^{(m)}\right) d s \\
& =\mathcal{N}_{i}^{r c} \mathcal{N}_{j}^{b f} \int_{0}^{h} \sin \left(k_{z, i}\left(z+b_{o}\right)\right) \Gamma_{l}(z) d z \int_{S_{C_{1}}} \nabla_{s} \xi_{i_{x_{c}} i_{y_{c}}} \cdot \nabla_{s} \Lambda_{p q}^{(m)} d s
\end{aligned}
$$

where $\mathcal{N}_{i}^{r c}$ and $\mathcal{N}_{j}^{b f}$ are, respectively, the normalization factors of the rectangular cavity mode and the basis function; $\nabla_{s}$ is the projection of the $\nabla$ operator in the plane defined by $\left(\widehat{u}_{\rho}, \widehat{u}_{\varphi}\right) ; S_{C_{1}}$ is the surface defined by the bottom cap of the cylinder; $k_{z_{c}, i}$ is the wavenumber related to the coordinate $z_{c}$ and associated to the $i$-th mode of the rectangular cavity; $\xi_{i_{x_{c}} i_{y_{c}}}$ is the scalar function used to calculate the $T E_{z_{c}}$ mode of the rectangular cavity (see Section A.2 of Appendix A).

Making use of the property (4.6), we have:

$$
S_{i j}=\mathcal{N}_{i}^{r c} \mathcal{N}_{j}^{b f} I_{i_{z_{c}}, l}(z)\left[\frac{d}{2} \oint_{\rho=\frac{d}{2}} \xi_{i_{x_{c}} i_{y_{c}}} \frac{\partial \Lambda_{p q}^{(m)}}{\partial \rho} d \varphi+\Delta_{p q}^{2} \int_{S_{C_{1}}} \xi_{i_{x_{c}} i_{y_{c}}} \Lambda_{p q}^{(m)} d s\right]
$$

where the following integral with analytical solution has been defined :

$$
I_{i_{z_{c}}, l}(z) \equiv \int_{0}^{h} \sin \left(k_{z_{c}, i}\left(z+b_{o}\right)\right) \Gamma_{l}(z) d z
$$


We can now distinguish between the cases in which the basis function is a perfect electric walls eigenvector or a perfect magnetic walls one.

Case basis function PEW:

In this case, taking into account that $\left.\frac{\partial \psi_{p q}^{(m)}}{\partial \rho}\right|_{\rho=\frac{d}{2}}=0$ holds due to the boundary conditions of derivative of the Bessel functions of first order, and introducing the scalar functions corresponding to the PEW case (see equation (4.21)) into (4.24), we obtain:

$$
\begin{aligned}
S_{i j} & =\mathcal{N}_{i}^{r c} \mathcal{N}_{j}^{b f} \Theta_{p q}^{\prime 2} I_{i_{z_{c}}, l}^{s s}(z) \int_{0}^{\frac{d}{2}} \rho d \rho J_{p}\left(\Theta_{p q}^{\prime} \rho\right) \\
& \int_{0}^{2 \pi} d \varphi \cos \left(k_{x_{c}, i}\left(\rho \cos \varphi+a_{o}\right)\right) \cos \left(k_{y_{c}, i}\left(\rho \sin \varphi+c_{o}\right)\right)\left\{\begin{array}{l}
\cos (p \varphi) \\
\sin (p \varphi)
\end{array}\right\}\left(\begin{array}{l}
m)=(1) \\
(m)=(2)
\end{array}\right.
\end{aligned}
$$

In this last expression, $k_{x_{c}, i}$ and $k_{y_{c}, i}$ represent the wavenumber related to the coordinates $x_{c}$ and $y_{c}$ respectively, associated to the $i$-th mode of the rectangular cavity (see eq. (A.28) of the Appendix A). Moreover, the integral in (4.26) is computed numerically, whereas the integral in (4.25) has analytical solution and is expressed now as:

$$
I_{i_{z_{c}}, l}^{s s}(z) \equiv \int_{0}^{h} \sin \left(k_{z_{c}, i}\left(z+b_{o}\right)\right) \sin \left(\beta_{l}^{h} z\right) d z
$$

\section{Case basis function PMW:}

Analogously, by introducing the PMW scalar functions (4.21) into (4.24), we have:

$$
S_{i j}=\mathcal{N}_{i}^{r c} \mathcal{N}_{j}^{b f} I_{i_{z_{c}}, l}^{s c}(z)\left\{\chi_{p q} J_{p}^{\prime}\left(\chi_{p q}\right) I_{i_{x_{c}}, i_{y_{c}}}^{(p, m)}(\varphi)+\Theta_{p q}^{2} I_{i_{x_{c}}, i_{y_{c}}}^{(p, q, m)}(\rho, \varphi)\right\}
$$

where the integrals $I_{i_{x_{c}}, i_{y_{c}}}^{(p, m)}(\varphi), I_{i_{x_{c}}, i_{y_{c}}}^{(p, q)}(\rho, \varphi)$ and $I_{i_{z_{c}}, l}^{s c}(z)$ are defined as:

$$
\begin{aligned}
& I_{i_{z_{c}}, l}^{s c}(z) \equiv \int_{0}^{h} \sin \left(k_{z_{c}, i}\left(z+b_{o}\right)\right) \cos \left(\beta_{l}^{h} z\right) d z \\
& I_{i_{x_{c}}, i_{y_{c}}}^{(p, m)}(\varphi) \equiv \int_{0}^{2 \pi} d \varphi \cos \left(k_{x_{c}, i}\left(\rho \cos \varphi+a_{o}\right)\right) \cos \left(k_{y_{c}, i}\left(\rho \sin \varphi+c_{o}\right)\right)\left\{\begin{array}{l}
\cos (p \varphi) \\
\sin (p \varphi)
\end{array}\right\} \begin{array}{l}
(m)=(1) \\
(m)=(2)
\end{array} \\
& I_{i_{x_{c}}, i_{y_{c}}}^{(p, q)}(\rho, \varphi) \equiv \int_{0}^{\frac{d}{2}} \rho d \rho J_{p}\left(\Theta_{p q} \rho\right) \\
& \int_{0}^{2 \pi} d \varphi \cos \left(k_{x_{c}, i}\left(\rho \cos \varphi+a_{o}\right)\right) \cos \left(k_{y_{c}, i}\left(\rho \sin \varphi+c_{o}\right)\right)\left\{\begin{array}{l}
\cos (p \varphi) \\
\sin (p \varphi)
\end{array}\right\} \begin{array}{l}
(m)=(1) \\
(m)=(2)
\end{array}
\end{aligned}
$$

The integral $I_{i_{z_{c}}, l}^{s c}(z)$ has analytical solution, whereas the integrals $I_{i_{x_{c}}, i_{y_{c}}}^{(p, m)}(\varphi), I_{i_{x_{c}}, i_{y_{c}}}^{(p, q, m)}(\rho, \varphi)$ 
are computed numerically.

\section{B. Case $\vec{E}_{i}$ is a $\mathbf{T} \mathbf{M}^{\mathbf{z}_{\mathrm{c}}}$ mode and $\vec{w}_{j}$ is a $\mathbf{T E}^{\mathbf{z}_{\mathrm{c}}}$ basis function}

Making use again of the notation defined by the equations (4.21) and introducing the expression (4.22), as well as the $T M^{z_{c}}$ solenoidal mode of the rectangular cavity (see equation (A.22)) in equation (4.20), we have:

$$
\begin{aligned}
S_{i j}= & \mathcal{N}_{i}^{r c} \mathcal{N}_{j}^{b f} \int_{V_{1}}\left[k_{z_{c}, i} \sin \left(k_{z_{c}, i}\left(z+b_{o}\right)\right) \nabla_{s} \eta_{i_{x_{c}} i_{y_{c}}}-k_{t, i}^{2} \cos \left(k_{z_{c}, i}\left(z+b_{o}\right)\right) \eta_{i_{x_{c}} i_{y_{c}}} \widehat{u}_{z}\right] . \\
& \cdot\left(\widehat{u}_{z} \times \nabla_{s} \Lambda_{p q}^{(m)}\right) \Gamma_{l}(z) d v= \\
= & \mathcal{N}_{i}^{r c} \mathcal{N}_{j}^{b f} k_{z_{c}, i} \int_{0}^{h} \sin \left(k_{z_{c}, i}\left(z+b_{o}\right)\right) \Gamma_{l}(z) d z \int_{S_{C_{1}}} \nabla_{s} \eta_{i_{x_{c} i_{y_{c}}}} \cdot\left(\widehat{u}_{z} \times \nabla_{s} \Lambda_{p q}^{(m)}\right) d s
\end{aligned}
$$

where $k_{t, i}$ is the transverse wavenumber associated to the $i$-th resonant mode of the rectangular cavity, and it is defined in (A.28). Next, applying the equation (4.14), we can write:

$$
S_{i j}=\mathcal{N}_{i}^{r c} \mathcal{N}_{j}^{b f} \beta_{t}^{c} \int_{0}^{h} \sin \left(k_{z_{c}, i}\left(z+b_{o}\right)\right) \Gamma_{l}(z) d z \oint_{\rho=\frac{d}{2}} \eta_{i_{x_{c}} i_{y_{c}}} \frac{\partial \Lambda_{p q}^{(m)}}{\partial \varphi} d \varphi
$$

This way, it is easy now to analyse the two different possibilities: PEW or PMW basis functions.

\section{Case basis function PEW:}

By introducing the scalar functions of the PEW basis function, from eq.(4.21), into the equation (4.30), we obtain the value of $S_{i j}$,

$$
\begin{aligned}
S_{i j} & =\mathcal{N}_{i}^{r c} \mathcal{N}_{j}^{b f} k_{z_{c}, i} p J_{p}\left(\chi_{p q}^{\prime}\right) \int_{0}^{h} \sin \left(k_{z_{c}, i}\left(z+b_{o}\right)\right) \sin \left(\beta_{l}^{h} z\right) d z \\
& \int_{0}^{2 \pi} d \varphi \sin \left(k_{x_{c}, i}\left(\frac{d}{2} \cos \varphi+a_{o}\right)\right) \sin \left(k_{y_{c}, i}\left(\frac{d}{2} \sin \varphi+c_{o}\right)\right)\left\{\begin{array}{c}
-\sin (p \varphi) \\
\cos (p \varphi)
\end{array}\right\} \begin{array}{l}
(m)=(1) \\
(m)=(2)
\end{array}
\end{aligned}
$$

Case basis function PMW:

In this case, since $\left.\frac{\partial \phi_{p q}^{(m)}}{\partial \psi}\right|_{\rho=\frac{d}{2}}$, we directly obtain from equation (4.30):

$$
S_{i j}=0 \quad \forall \quad(m)=(1),(2)
$$

\section{Case $\vec{E}_{i}$ is a $\mathbf{T E} E^{\mathbf{z}_{\mathbf{c}}}$ mode and $\vec{w}_{j}$ is a $\mathbf{T} \mathbf{M}^{\mathrm{zc}}$ basis function}

Up to now the entries of the $\mathbf{S}$ matrix with $\vec{w}_{j}$ being a $T E^{z_{c}}$ basis functions, have been evaluated. The use of the equations (4.21) has simplified our calculus, since they permit 
to consider at the same time PEW and PMW eigenvectors. Next, to proceed analogously with the calculation of the cases where $\vec{w}_{j}$ is a $T M^{z_{c}}$ basis functions, we use the following notation:

$$
\begin{aligned}
& \Gamma_{l}^{(1)}(z) \equiv\left\{\begin{array}{c}
\sin \left(\beta_{l}^{h} z\right) \\
\cos \left(\beta_{l}^{h} z\right)
\end{array}\right\} ; \quad \Delta_{p q} \equiv\left\{\begin{array}{c}
\Theta_{p q} \\
\Theta_{p q}^{\prime}
\end{array}\right\} ; \quad \begin{array}{l}
\vec{w}_{j} \in P E W \\
\vec{w}_{j} \in P M W
\end{array}
\end{aligned}
$$

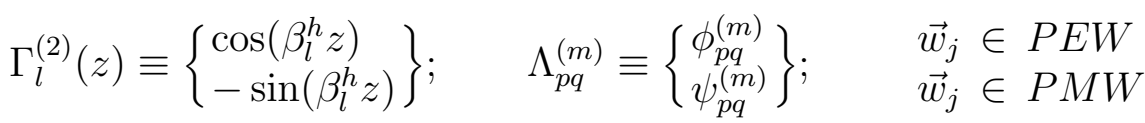

where $\beta_{h}^{l} \equiv \frac{l \pi}{h}$ and the scalar functions $\Theta_{p q}^{\prime}, \Theta_{p q}, \psi_{p q}^{(m)}, \phi_{p q}^{(m)}$ are defined in Table 4.1.

This way, we can express a $T M^{z_{c}}$ basis function, independently whether it is solution of the PEW or PMW cylindrical cavity, as follows:

$$
\vec{w}_{j}(\vec{r})=\mathcal{N}_{j}^{b f}\left[\beta_{l}^{h} \Gamma_{l}^{(1)}(z) \nabla_{s} \Lambda_{p q}^{(m)}-\Delta_{p q}^{2} \Gamma_{l}^{(2)}(z) \Lambda_{p q}^{(m)} \widehat{u}_{z}\right]
$$

Hence, introducing the expression (4.34) and the $T E^{z_{c}}$ solenoidal mode of the empty rectangular cavity (see equation (A.22)) in equation (4.20), we can write:

$$
\begin{aligned}
S_{i j} & =\mathcal{N}_{i}^{r c} \mathcal{N}_{j}^{b f} \int_{V_{1}} \sin \left(k_{z_{c}, i}\left(z+b_{o}\right)\right)\left(\widehat{u}_{z} \times \nabla_{s} \xi_{i_{x_{c}} i_{y_{c}}}\right) \cdot\left[\beta_{l}^{h} \Gamma_{l}^{(1)}(z) \nabla_{s} \Lambda_{p q}^{(m)}-\Delta_{p q}^{2} \Gamma_{l}^{(2)}(z) \Lambda_{p q}^{(m)} \widehat{u}_{z}\right] d v \\
& =\mathcal{N}_{i}^{r c} \mathcal{N}_{j}^{b f} \beta_{l}^{h} \int_{0}^{h} \sin \left(k_{z_{c}, i}\left(z+b_{o}\right)\right) \Gamma_{l}^{(1)}(z) d z \int_{S_{C_{1}}}\left(\nabla_{s} \Lambda_{p q}^{(m)} \times \widehat{u}_{z}\right) \cdot \nabla_{s} \xi_{i_{x_{c}} i_{y_{c}}} d s
\end{aligned}
$$

and applying the equation (4.14), we have:

$$
S_{i j}=-\mathcal{N}_{i}^{r c} \mathcal{N}_{j}^{b f} \beta_{l}^{h} \int_{0}^{h} \sin \left(k_{z_{c}, i}\left(z+b_{o}\right)\right) \Gamma_{l}^{(1)}(z) d z \oint_{\rho=\frac{d}{2}} \xi_{i_{x_{c}} i_{y_{c}}} \frac{\partial \Lambda_{p q}^{(m)}}{\partial \psi} d \varphi
$$

Thus, we can easily evaluate this expression for PEW or PMW cavity basis functions:

Case basis function PEW:

Since $\left.\frac{\partial \phi_{p q}^{(m)}}{\partial \psi}\right|_{\rho=\frac{d}{2}}$, we directly obtain from equation (4.36):

$$
S_{i j}=0 \quad \forall \quad(m)=(1),(2)
$$

Case basis function PMW:

By introducing equations (4.33b) into (4.36), we have:

$$
\begin{aligned}
S_{i j} & =-\mathcal{N}_{i}^{r c} \mathcal{N}_{j}^{b f} \beta_{l}^{h} p J_{p}\left(\chi_{p q}^{\prime}\right) \int_{0}^{h} \sin \left(k_{z_{c}, i}\left(z+b_{o}\right)\right) \cos \left(\beta_{l}^{h} z\right) d z \\
& \int_{0}^{2 \pi} d \varphi \cos \left(k_{x_{c}, i}\left(\frac{d}{2} \cos \varphi+a_{o}\right)\right) \cos \left(k_{y_{c}, i}\left(\frac{d}{2} \sin \varphi+c_{o}\right)\right)\left\{\begin{array}{c}
-\sin (p \varphi) \\
\cos (p \varphi)
\end{array}\right\} \begin{array}{l}
(m)=(1) \\
(m)=(2)
\end{array}
\end{aligned}
$$




\section{Case $\vec{E}_{i}$ is a $\mathbf{T} \mathbf{M}^{\mathbf{z}_{\mathrm{c}}}$ mode and $\vec{w}_{j}$ is a $\mathbf{T} \mathbf{M}^{\mathbf{z}_{\mathfrak{c}}}$ basis function}

In this case, by introducing the expression (4.34) and the $T M^{z_{c}}$ solenoidal mode of the rectangular cavity (see equation (A.22)) in equation (4.20), we have:

$$
\begin{aligned}
S_{i j}=\mathcal{N}_{i}^{r c} \mathcal{N}_{j}^{b f} \int_{V_{1}}\left[k_{z_{c}, i} \sin \left(k_{z_{c}, i}\left(z+b_{o}\right)\right) \nabla_{s} \eta_{i_{x_{c}} i_{y_{c}}}-k_{t, i}^{2} \cos \left(k_{z_{c}, i}\left(z+b_{o}\right)\right) \eta_{i_{x_{c}} i_{y_{c}}} \widehat{u}_{z}\right] . \\
\cdot\left[\beta_{l}^{h} \Gamma_{l}^{(1)}(z) \nabla_{s} \Lambda_{p q}^{(m)}-\Delta_{p q}^{2} \Gamma_{l}^{(2)}(z) \Lambda_{p q}^{(m)} \widehat{u}_{z}\right] d v \\
=\mathcal{N}_{i}^{r c} \mathcal{N}_{j}^{b f}\left[k_{z_{c}, i} \beta_{l}^{h} I_{i_{z_{c}}, l}^{(1)}(z) \int_{S_{C_{1}}} \nabla_{s} \eta_{i_{x_{c}} i_{y_{c}}} \cdot \nabla_{s} \Lambda_{p q}^{(m)} d s+k_{t, i}^{2} \Delta_{p q}^{2} I_{i_{z_{c}}, l}^{(2)}(z) \int_{S_{C_{1}}} \eta_{i_{x_{c}} i_{y_{c}}} \Lambda_{p q}^{(m)} d s\right]
\end{aligned}
$$

where the integrals $I_{i_{z_{c}}, l}^{(1)}(z)$ and $I_{i_{z_{c}}, l}^{(2)}(z)$ have analytical solution, and have been defined as:

$$
\begin{aligned}
& I_{i_{z_{c}}, l}^{(1)}(z) \equiv \int_{0}^{h} \sin \left(k_{z_{c}, i}\left(z+b_{o}\right)\right) \Gamma_{l}^{(1)}(z) d z \\
& I_{i_{z_{c}}, l}^{(2)}(z) \equiv \int_{0}^{h} \cos \left(k_{z_{c}, i}\left(z+b_{o}\right)\right) \Gamma_{l}^{(2)}(z) d z
\end{aligned}
$$

Furthermore, by using the expression (4.6), we can rewrite the equation (4.39) as:

$$
\begin{aligned}
S_{i j}=\mathcal{N}_{i}^{r c} \mathcal{N}_{j}^{b f}\{ & k_{z_{c}, i} \beta_{l}^{h} I_{i_{z_{c}}, l}^{(1)}(z) I_{i_{x_{c}}, i_{y_{c}}}^{(p, q)}(\varphi)+ \\
& \left.+\left[k_{z_{c}, i} \beta_{l}^{h} \Delta_{p q}^{2} I_{i_{z_{c}}, l}^{(1)}(z)+k_{t, i}^{2} \Delta_{p q}^{2} I_{i_{z_{c}}, l}^{(2)}(z)\right] I_{i_{x_{c}}, i_{y_{c}}}^{(p, q, m)}(\rho, \varphi)\right\}
\end{aligned}
$$

where we have defined:

$$
\begin{aligned}
& \left.I_{i_{x_{c}}, i_{y_{c}}}^{(p, q) m)}(\varphi) \equiv \int_{0}^{2 \pi} \eta_{i_{x_{c}} i_{y_{c}}} \frac{\partial \Lambda_{p q}^{(m)}}{\partial \rho}\right|_{\rho=d / 2} d \varphi \\
& I_{i_{x_{c}}, i_{y_{c}}}^{(p, q, m)}(\rho, \varphi) \equiv \int_{S_{C_{1}}} \eta_{i_{x_{c}} i_{y_{c}}} \Lambda_{p q}^{(m)} d s
\end{aligned}
$$

Therefore to calculate the entry of the $\mathbf{S}$ matrix in this case, we have to evaluate the integrals $I_{i_{z_{c}}, l}^{(1)}(z), I_{i_{z_{c}}, l}^{(2)}(z), I_{i_{x_{c}}, i_{y_{c}}}^{(p, q, m)}(\varphi)$ and $I_{i_{x_{c}}, i_{y_{c}}}^{(p, q, m)}(\rho, \varphi)$ and introduce them in equation (4.41).

Case basis function PEW:

By introducing the expressions (4.33b) for PEW basis functions into the equations (4.42) 
and (4.40), we obtain

$$
\begin{aligned}
& I_{i_{z_{c}}, l}^{(1)}(z)=\int_{0}^{h} \sin \left(k_{z_{c}, i}\left(z+b_{o}\right)\right) \sin \left(\beta_{l}^{h} z\right) d z=I_{i_{z_{c}}, l}^{s s}(z) \\
& I_{i_{z_{c}}, l}^{(2)}(z)=\int_{0}^{h} \cos \left(k_{z_{c}, i}\left(z+b_{o}\right)\right) \cos \left(\beta_{l}^{h} z\right) d z=I_{i_{z_{c}}, l}^{c c}(z)
\end{aligned}
$$

which have analytical solution, and,

$$
\begin{aligned}
& I_{i_{x_{c}}, i_{y_{c}}}^{(p, q, m)}(\varphi)=\chi_{p q} J_{p}^{\prime}\left(\chi_{p q}\right) \\
& \int_{0}^{2 \pi} d \varphi \sin \left(k_{x_{c}, i}\left(\frac{d}{2} \cos \varphi+a_{o}\right)\right) \sin \left(k_{y_{c}, i}\left(\frac{d}{2} \sin \varphi+c_{o}\right)\right)\left\{\begin{array}{c}
\cos (p \varphi) \\
\sin (p \varphi)
\end{array}\right\} \begin{array}{l}
(m)=(1) \\
(m)=(2)
\end{array} \\
& I_{i_{x_{c}}, i_{y_{c}}}^{(p, m)}(\rho, \varphi)=\int_{0}^{\frac{d}{2}} \rho J_{p}\left(\Theta_{p q} \rho\right) d \rho \\
& \int_{0}^{2 \pi} d \varphi \sin \left(k_{x_{c}, i}\left(\frac{d}{2} \cos \varphi+a_{o}\right)\right) \sin \left(k_{y_{c}, i}\left(\frac{d}{2} \sin \varphi+c_{o}\right)\right)\left\{\begin{array}{l}
\cos (p \varphi) \\
\sin (p \varphi)
\end{array}\right\} \begin{array}{l}
(m)=(1) \\
(m)=(2)
\end{array}
\end{aligned}
$$

These last expressions are computed numerically.

Case basis function PMW:

Since $\left.\frac{\partial \psi_{p q}^{(m)}}{\partial \rho}\right|_{\rho=\frac{d}{2}} ^{=0,}$ in this case we have:

$$
I_{i_{x_{c}}, i_{y_{c}}}^{(p, q, m)}(\varphi)=0 \quad \forall(m)=(1),(2)
$$

whereas the rest of integrals are:

$$
\begin{aligned}
& I_{i_{z_{c}}, l}^{(1)}(z)=\int_{0}^{h} \sin \left(k_{z_{c}, i}\left(z+b_{o}\right)\right) \cos \left(\beta_{l}^{h} z\right) d z=I_{i_{z_{c}}, l}^{s c}(z) \\
& I_{i_{z_{c}}, l}^{(2)}(z)=-\int_{0}^{h} \cos \left(k_{z_{c}, i}\left(z+b_{o}\right)\right) \sin \left(\beta_{l}^{h} z\right) d z=-I_{i_{z_{c}}, l}^{c s}(z)
\end{aligned}
$$

with analytical solution, and,

$$
\begin{aligned}
& I_{i_{x_{c}}, i_{y_{c}}}^{(p, q, m)}(\rho, \varphi)=\int_{0}^{\frac{d}{2}} \rho J_{p}\left(\Theta_{p q}^{\prime} \rho\right) d \rho \\
& \int_{0}^{2 \pi} d \varphi \sin \left(k_{x_{c}, i}\left(\frac{d}{2} \cos \varphi+a_{o}\right)\right) \sin \left(k_{y_{c}, i}\left(\frac{d}{2} \sin \varphi+c_{o}\right)\right)\left\{\begin{array}{l}
\cos (p \varphi) \\
\sin (p \varphi)
\end{array}\right\} \begin{array}{l}
(m)=(1) \\
(m)=(2)
\end{array}
\end{aligned}
$$

which is computed numerically. 


\subsubsection{Computation of the $\mathrm{C}$ matrix}

The $\mathbf{C}$ matrix is a real symmetric matrix whose entries are:

$$
C_{i j}=\int_{S_{1}} \int_{S_{1}}\left(\vec{w}_{i}(\vec{r}) \cdot \widehat{n}\right) g^{e}\left(\vec{r}, \vec{r}^{\prime}\right)\left(\vec{w}_{j}\left(\vec{r}^{\prime}\right) \cdot \widehat{n}^{\prime}\right) d s d s^{\prime}
$$

where $S_{1}$ is the surface of the cylindrical dielectric resonator, $\vec{w}_{i}(\vec{r})$ and $\vec{w}_{j}\left(\vec{r}^{\prime}\right)$ are the basis functions, $g^{e}\left(\vec{r}, \vec{r}^{\prime}\right)$ is the scalar Green's function for the electric scalar potential of the empty rectangular cavity, $\vec{r}$ and $\vec{r}^{\prime}$ are, respectively, the observation and the source points. We recall that $i, j=1,2, \ldots, Q$, being $Q$ the number of basis functions used to expand the polarization vector by the method of moments.

The first conclusion that we can extract from equation (4.51) is that the contribution of the PMW basis functions to the $\mathbf{C}$ matrix is null. This is due to the fact that the normal component to the cylindrical cavity of this kind of basis functions is equal to zero. In fact, it results evident from the discussion in section 4.2, since the $\mathbf{C}$ matrix represents somehow the contribution of the equivalent electric polarization charge density described by equation (3.17).

Hence,

$$
C_{i j}=0 \quad \forall \vec{w}_{i}, \vec{w}_{j} \quad \in \quad \mathbf{P M W}\{\overrightarrow{\mathbf{w}}\}
$$

Thus, to evaluate the $\mathbf{C}$ matrix we will focus our attention in the entries where both $\vec{w}_{i}$ and $\vec{w}_{j}$ are basis functions solution of the PEW cylindrical cavity. Furthermore, in order to calculate it, the surface $S_{1}$ has been divided into three parts:

- $S_{1}^{(1)}$ denotes the bottom cap, and its normal outward vector is: $\widehat{n}^{(1)}=-\widehat{u}_{z}$

- $S_{1}^{(2)}$ denotes the top cap, and its normal outward vector is: $\widehat{n}^{(2)}=\widehat{u}_{z}$

- $S_{1}^{(3)}$ denotes the lateral surface, and its normal outward vector is: $\widehat{n}^{(3)}=\widehat{u}_{\rho}$

The total surface of the dielectric puck may be expressed as:

$$
S_{1}=S_{1}^{(1)} \cup S_{1}^{(2)} \cup S_{1}^{(3)}
$$

Furthermore, by defining the following matrices:

$$
C_{i j}^{(\alpha, \beta)} \equiv \int_{S_{1}^{(\alpha)}} \int_{S_{1}^{(\beta)}}\left(\vec{w}_{i}(\vec{r}) \cdot \widehat{n}^{(\alpha)}\right) g^{e}\left(\vec{r}, \vec{r}^{\prime}\right)\left(\vec{w}_{j}\left(\vec{r}^{\prime}\right) \cdot \widehat{n}^{(\beta) \prime}\right) d s d s^{\prime}
$$

where $\alpha, \beta=1,2,3$. The entry of the $\mathbf{C}$ matrix can be expressed as:

$$
C_{i j}=\sum_{\alpha, \beta=1}^{3} C_{i j}^{(\alpha, \beta)}
$$

This way, in order to compute the $\mathbf{C}$ matrix we have to evaluate 9 submatrices. However, due to the fact that $g^{e}\left(\vec{r}, \vec{r}^{\prime}\right)=g^{e}\left(\vec{r}^{\prime}, \vec{r}\right)$, we have

$$
\mathbf{C}^{(\alpha, \beta)}=\mathbf{C}_{T}^{(\beta, \alpha)} \quad \Rightarrow \quad C_{i j}^{(\alpha, \beta)}=C_{j i}^{(\beta, \alpha)}
$$


Therefore, we only need to calculate:

$$
\mathbf{C}^{(\alpha, \beta)} \quad \text { with } \quad \beta \geq \alpha
$$

The evaluation of these matrices must be performed carefully, since, as mentioned in the previous chapters, the scalar Green's function is singular when $\vec{r}=\vec{r}^{\prime}$. Indeed, a first inspection of the expression (4.54) suggest us to divide the matrices into two different types: a former kind of matrices that involve integrals in which the observation and the source point do not coalesce. Hence, the integrals to be solved do not present any singularity. The second kind are those matrices whose entries involve singular integrals due to the fact that $\vec{r}$ and $\vec{r}^{\prime}$ coalesce.

The matrix $\mathbf{C}^{(\mathbf{1 , 2})}$ is of the first kind, since

$$
\vec{r} \neq \vec{r}^{\prime} \quad \forall \quad \vec{r} \in S_{1}^{(1)} ; \quad \vec{r}^{\prime} \in S_{1}^{(2)}
$$

This matrix is computed numerically and for this purpose the scalar Green's function is efficiently calculated by means of the Ewald technique [102] (for details, see [101], [74], [Ch. 3, 103] or [104]).

The second group is made up of the matrices $\mathbf{C}^{(\mathbf{1 , 1})}, \mathbf{C}^{(\mathbf{2 , 2})}, \mathbf{C}^{(\mathbf{3 , 3})}, \mathbf{C}^{(\mathbf{1 , 3})}$ and $\mathbf{C}^{(\mathbf{2 , 3})}$. In order to calculate them, we extract the singularity of the $g^{e}$ in closed form using the expression (2.79).

$$
g^{e}\left(\vec{r}, \vec{r}^{\prime}\right)=\frac{1}{4 \pi R}+g_{r}^{e}\left(\vec{r}, \vec{r}^{\prime}\right)
$$

Thus, we have to calculate each matrix as the sum of two matrices: the former one containing the singularity of the Green's function and the last one which involves regular integrals.

$$
\mathbf{C}^{(\alpha, \beta)}=\tilde{\mathbf{C}}^{(\alpha, \beta)}+\mathbf{C}_{\mathbf{r}}^{(\alpha, \beta)}
$$

being,

$$
\begin{aligned}
& \tilde{C}_{i j}^{(\alpha, \beta)}=\int_{S_{1}^{(\alpha)}} \int_{S_{1}^{(\beta)}}\left(\vec{w}_{i}(\vec{r}) \cdot \widehat{n}^{(\alpha)}\right) \frac{1}{4 \pi R}\left(\vec{w}_{j}\left(\vec{r}^{\prime}\right) \cdot \widehat{n}^{(\beta) \prime}\right) d s d s^{\prime} \\
& C_{r, i j}^{(\alpha, \beta)}=\int_{S_{1}^{(\alpha)}} \int_{S_{1}^{(\beta)}}\left(\vec{w}_{i}(\vec{r}) \cdot \widehat{n}^{(\alpha)}\right) g_{r}^{e}\left(\vec{r}, \vec{r}^{\prime}\right)\left(\vec{w}_{j}\left(\vec{r}^{\prime}\right) \cdot \widehat{n}^{(\beta) \prime}\right) d s d s^{\prime}
\end{aligned}
$$

where $R=\left|\vec{r}-\vec{r}^{\prime}\right|$, and $g_{r}^{e}\left(\vec{r}, \vec{r}^{\prime}\right)$ is the regular part of the scalar Green's function.

The regular matrices $\mathbf{C}_{\mathbf{r}}^{(\alpha, \beta)}$ are computed numerically, using the Ewald's technique to calculate the scalar Green's function, like for the $\mathbf{C}^{(\mathbf{1 , 2})}$ matrix. On the other hand, the matrices involving singular integrals must be treated analytically to transform them into integrable expressions. In the following paragraphs, we explain how to proceed with the different $\tilde{\mathbf{C}}^{(\alpha, \beta)}$ matrices.

All the calculus are related to the cylindrical coordinate system placed in the center of the bottom cap of the dielectric puck. Furthermore, we assume that the modal indexes 
$\{p, q, l, m\}$ are associated to the $\vec{w}_{i}$ basis function, whereas the modal indexes $\{u, v, t, n\}$ are associated to $\vec{w}_{j}$.

\section{A. Computation of matrix $\tilde{\mathbf{C}}^{(1,1)}$}

In this case, the entry of the $\tilde{\mathbf{C}}^{(\alpha, \beta)}$ matrix is:

$$
\tilde{C}_{i j}^{(1,1)}=\int_{S_{1}^{(1)}} \int_{S_{1}^{(1)}}\left(-\vec{w}_{i}(\vec{r}) \mid \cdot \widehat{u}_{z=0}\right) \frac{1}{4 \pi R}\left(-\left.\vec{w}_{j}\left(\vec{r}^{\prime}\right)\right|_{z^{\prime}=0} \cdot \widehat{u}_{z}\right) d s d s^{\prime}
$$

In order to evaluate this expression, the first step is to introduce into equation (4.59) the expressions of the $T E^{z}$ and $T M^{z}$ basis functions of the PEW cavity.

Case $\vec{w}_{i}$ or $\vec{w}_{j}$ are $T E^{z}$ basis function:

Since the z-component of a $T E^{z}$ basis function is

$$
\left(\vec{w}_{i}(\vec{r}) \mid \widehat{u}_{z=0}\right)=0
$$

then we have:

$$
\tilde{C}_{i j}^{(1,1)}=0
$$

Case $\vec{w}_{i}$ and $\vec{w}_{j}$ are $T M^{z}$ basis function:

The normal component to $S_{1}^{(1)}$ of the $T M^{z}$ basis function is given by:

$$
\left(\vec{w}_{i}(\vec{r}) \mid \cdot \widehat{u}_{z=0}\right)=-\mathcal{N}_{i} \Theta_{p q}^{2} \phi_{p q}^{(m)}
$$

By introducing this expression into the equation (4.59), we have:

$$
\tilde{C}_{i j}^{(1,1)}=\mathcal{N}_{i} \mathcal{N}_{j} \frac{\Theta_{p q}^{2} \Theta_{u v}^{2}}{4 \pi} \int_{S_{1}^{(1)}} \int_{S_{1}^{(1)}} \frac{\phi_{p q}^{(m)}(\vec{r}) \phi_{u v}^{(n)}\left(\vec{r}^{\prime}\right)}{R} d s d s^{\prime}
$$

The integral in (4.63) is singular when $\vec{r}=\vec{r}^{\prime}$. To avoid this singularity, we make use of one of the results obtained from the work of P. Arcioni et al. [106], that permits to express the term $1 / R$ as:

$$
\frac{1}{R}=-\nabla_{s} \cdot \nabla_{s}^{\prime} R
$$

where $\nabla_{s}$ is the surface nabla operator. It is remarkable that this result is only applicable when $\vec{r}$ and $\vec{r}^{\prime}$ are on a coplanar surface.

This way, introducing the expression (4.64) into equation (4.63), and making use of the equation (D.10) (see Appendix D), we can rewrite:

$$
\tilde{C}_{i j}^{(1,1)}=\mathcal{N}_{i} \mathcal{N}_{j} \frac{\Theta_{p q}^{2} \Theta_{u v}^{2}}{4 \pi}\left(I_{p q, u v}^{(1)}-I_{p q, u v}^{(2)}-I_{p q, u v}^{(3)}+I_{p q, u v}^{(4)}\right)
$$


where the following integrals have been defined:

$$
\begin{aligned}
& I_{p q, u v}^{(1)}=\left.\left.\frac{d^{2}}{4} \oint_{o}^{2 \pi} \oint_{o}^{2 \pi} \phi_{p q}^{(m)}(\vec{r})\right|_{\rho=\frac{d}{2}} \phi_{u v}^{(n)}\left(\vec{r}^{\prime}\right)\right|_{\rho^{\prime}=\frac{d}{2}} R\left(\widehat{u}_{\rho} \cdot \widehat{u}_{\rho}^{\prime}\right) d \varphi d \varphi^{\prime} \\
& I_{p q, u v}^{(2)}=\left.\frac{d}{2} \int_{S_{1}^{(1)}} \oint_{o}^{2 \pi} \phi_{p q}^{(m)}(\vec{r})\right|_{\rho=\frac{d}{2}} R\left(\widehat{u}_{\rho}^{\prime} \cdot \nabla_{s}^{\prime} \phi_{u v}^{(n)}\left(\vec{r}^{\prime}\right)\right) d \varphi d s^{\prime} \\
& I_{p q, u v}^{(3)}=\left.\frac{d}{2} \int_{S_{1}^{(1)}} \oint_{o}^{2 \pi} \phi_{u v}^{(n)}\left(\vec{r}^{\prime}\right)\right|_{\rho^{\prime}=\frac{d}{2}} R\left(\widehat{u}_{\rho} \cdot \nabla_{s} \phi_{p q}^{(m)}(\vec{r})\right) d \varphi^{\prime} d s \\
& I_{p q, u v}^{(4)}=\int_{S_{1}^{(1)}} \int_{S_{1}^{(1)}} R \nabla_{s} \phi_{p q}^{(m)}(\vec{r}) \cdot \nabla_{s}^{\prime} \phi_{u v}^{(n)}\left(\vec{r}^{\prime}\right) d s d s^{\prime}
\end{aligned}
$$

Since $\left.\phi_{p q}^{(m)}\right|_{\rho=\frac{d}{2}} ^{=} 0$ for any value of $p, q, m$, it can be easily observed that the integrals $I^{(1)}$, $I^{(2)}$ and $I^{(3)}$ defined above are equal to zero, whereas the expression (4.66d) can be further developed,

$$
\begin{aligned}
I_{p q, u v}^{(4)}= & \int_{S_{1}^{(1)}} \int_{S_{1}^{(1)}} R\left[\frac{\partial \phi_{p q}^{(m)}}{\partial \rho} \frac{\partial \phi_{u v}^{(n)}}{\partial \rho^{\prime}} \widehat{u}_{\rho} \cdot \widehat{u}_{\rho}^{\prime}+\frac{1}{\rho^{\prime}} \frac{\partial \phi_{p q}^{(m)}}{\partial \rho} \frac{\partial \phi_{u v}^{(n)}}{\partial \varphi^{\prime}} \widehat{u}_{\rho} \cdot \widehat{u}_{\varphi}^{\prime}+\right. \\
& \left.+\frac{1}{\rho} \frac{\partial \phi_{p q}^{(m)}}{\partial \varphi} \frac{\partial \phi_{u v}^{(n)}}{\partial \rho} \widehat{u}_{\varphi} \cdot \widehat{u}_{\rho}^{\prime}+\frac{1}{\rho \rho^{\prime}} \frac{\partial \phi_{p q}^{(m)}}{\partial \varphi} \frac{\partial \phi_{u v}^{(n)}}{\partial \varphi^{\prime}} \widehat{u}_{\varphi} \cdot \widehat{u}_{\varphi}^{\prime}\right] d s d s^{\prime} \\
= & I^{(41)}+I^{(42)}+I^{(43)}+I^{(44)}
\end{aligned}
$$

Next, taking into account that in cylindrical coordinates we have:

$$
\begin{gathered}
\widehat{u}_{\rho} \cdot \widehat{u}_{\rho}^{\prime}=\widehat{u}_{\varphi} \cdot \widehat{u}_{\varphi}^{\prime}=\cos \left(\varphi-\varphi^{\prime}\right) \\
\widehat{u}_{\rho} \cdot \widehat{u}_{\varphi}^{\prime}=-\widehat{u}_{\varphi} \cdot \widehat{u}_{\rho}^{\prime}=\sin \left(\varphi-\varphi^{\prime}\right) \\
R=\sqrt{\rho^{2}+\rho^{\prime 2}-2 \rho \rho^{\prime} \cos \left(\varphi-\varphi^{\prime}\right)}
\end{gathered}
$$

Finally, by introducing the expressions (4.68) and the values of the scalar functions from the Table 4.1 into the equation (4.67), we obtain the value of the $\tilde{\mathbf{C}}^{(\mathbf{1 , 1})}$ matrix in terms of regular integrals:

$$
\tilde{C}_{i j}^{(1,1)}=\mathcal{N}_{i} \mathcal{N}_{j} \frac{\Theta_{p q}^{2} \Theta_{u v}^{2}}{4 \pi}\left(I^{(41)}+I^{(42)}+I^{(43)}+I^{(44)}\right)
$$

with

$$
\begin{aligned}
I^{(41)}= & \Theta_{p q} \Theta_{u v} \delta_{p u} \delta_{m n} \int_{0}^{\frac{d}{2}} \int_{0}^{\frac{d}{2}} d \rho d \rho^{\prime} \rho \rho^{\prime} J_{p}^{\prime}\left(\Theta_{p q} \rho\right) J_{u}^{\prime}\left(\Theta_{u v} \rho^{\prime}\right) \\
& \oint_{0}^{2 \pi} \cos (\varphi) \cos (p \varphi) d \varphi \sqrt{\rho^{2}+\rho^{\prime 2}-2 \rho \rho^{\prime} \cos (\varphi)}\left\{\begin{array}{l}
\left(1+\delta_{0 p}\right) \pi \\
\left(1-\delta_{0 p}\right) \pi
\end{array}\right\} \begin{array}{l}
(m)=(1) \\
(m)=(2)
\end{array}
\end{aligned}
$$




$$
\begin{aligned}
I^{(42)}= & \Theta_{p q} u \delta_{p u} \delta_{m n} \int_{0}^{\frac{d}{2}} \int_{0}^{\frac{d}{2}} d \rho d \rho^{\prime} \rho J_{p}^{\prime}\left(\Theta_{p q} \rho\right) J_{u}\left(\Theta_{u v} \rho^{\prime}\right) \\
& \oint_{0}^{2 \pi} \sin (\varphi) \sin (p \varphi) d \varphi \sqrt{\rho^{2}+\rho^{\prime 2}-2 \rho \rho^{\prime} \cos (\varphi)}\left\{\begin{array}{c}
\left(1-\delta_{0 p}\right) \pi \\
\pi
\end{array}\right\} \begin{array}{l}
(m)=(1) \\
(m)=(2)
\end{array} \\
I^{(43)}= & \Theta_{u v} p \delta_{p u} \delta_{m n} \int_{0}^{\frac{d}{2}} \int_{0}^{\frac{d}{2}} d \rho d \rho^{\prime} \rho^{\prime} J_{p}\left(\Theta_{p q} \rho\right) J_{u}^{\prime}\left(\Theta_{u v} \rho^{\prime}\right) \\
& \oint_{0}^{2 \pi} \sin (\varphi) \sin (p \varphi) d \varphi \sqrt{\rho^{2}+\rho^{\prime 2}-2 \rho \rho^{\prime} \cos (\varphi)}\left\{\begin{array}{c}
\pi \\
\left(1-\delta_{0 p}\right) \pi
\end{array}\right\} \begin{array}{l}
(m)=(1) \\
(m)=(2)
\end{array} \\
I^{(44)}= & p u \delta_{p u} \delta_{m n} \int_{0}^{\frac{d}{2}} \int_{0}^{\frac{d}{2}} d \rho d \rho^{\prime} J_{p}\left(\Theta_{p q} \rho\right) J_{u}\left(\Theta_{u v} \rho^{\prime}\right) \\
& \oint_{0}^{2 \pi} \cos (\varphi) \cos (p \varphi) d \varphi \sqrt{\rho^{2}+\rho^{\prime 2}-2 \rho \rho^{\prime} \cos (\varphi)}\left\{\begin{array}{c}
\left(1-\delta_{0 p}\right) \pi \\
\left(1+\delta_{0 p}\right) \pi
\end{array}\right\} \begin{array}{l}
(m)=(1) \\
(m)=(2)
\end{array}
\end{aligned}
$$

All these expressions are calculated numerically. Note that to achieve the results presented above, we have used the technique described in the Appendix B (see (B.1)) to reduce the integration over the $\varphi$ and $\varphi^{\prime}$ variables. Therefore, not only the initial singular integrals have been transformed to regular ones, but also we have reduced the integrations involved from 4-dimensions to 3-dimensions.

\section{B. Computation of matrix $\tilde{\mathbf{C}}^{(2,2)}$}

The entries of the $\tilde{\mathbf{C}}^{(\mathbf{2 , 2})}$ matrix are:

$$
\tilde{C}_{i j}^{(2,2)}=\int_{S_{1}^{(2)}} \int_{S_{1}^{(2)}}\left(\left.\vec{w}_{i}(\vec{r})\right|_{z=h} ^{\cdot \widehat{u}_{z}}\right) \frac{1}{4 \pi R}\left(\left.\vec{w}_{j}\left(\vec{r}^{\prime}\right)\right|_{z^{\prime}=h} \widehat{u}_{z}\right) d s d s^{\prime}
$$

We can observe that the only difference between this expression and the equation (4.59) is the value of the $\mathrm{z}$-component of the basis functions at $z=0$ and at $z=h$, which is:

$$
\left.\vec{w}_{i}(\vec{r})\right|_{z=h} \cdot \widehat{u}_{z}=\left.(-1)^{l} \vec{w}_{i}(\vec{r})\right|_{z=0} \widehat{u}_{z} \quad \forall i ; \quad \text { with } i \equiv\{p, q, l\},(m)
$$

Therefore, if we assume that the modal indexes $\{p, q, l\}$ and $(m)$ are associated to the $\vec{w}_{i}$ basis function, and the modal indexes $\{u, v, t\}$ and $(n)$ are associated to $\vec{w}_{j}$, we have:

$$
\tilde{C}_{i j}^{(2,2)}=(-1)^{l+t} \tilde{C}_{i j}^{(1,1)}
$$

\section{Computation of matrix $\tilde{\mathbf{C}}^{(3,3)}$}

In order to calculate the $\tilde{\mathbf{C}}^{(\mathbf{3 , 3})}$ matrix, we have to evaluate the following expression:

$$
\tilde{C}_{i j}^{(3,3)}=\int_{S_{1}^{(3)}} \int_{S_{1}^{(3)}}\left(\left.\vec{w}_{i}(\vec{r})\right|_{\rho=\frac{d}{2}} \cdot \widehat{u}_{\rho}\right) \frac{1}{4 \pi R}\left(\left.\vec{w}_{j}\left(\vec{r}^{\prime}\right)\right|_{\substack{\cdot{ }^{\prime}=\frac{d}{2} \\ .}} ^{\prime} \widehat{u}_{\rho}^{\prime}\right) d s d s^{\prime}
$$


In this case, in order to avoid the singularity of the Green's function we can not use the expression (4.64) since $\vec{r}$ and $\vec{r}^{\prime}$ are not coplanar. Hence, we need to transform the singular integrals to regular ones using a different procedure from the followed for the $\tilde{\mathbf{C}}^{(\mathbf{1 , 1})}$ and the $\tilde{\mathbf{C}}^{(2,2)}$ matrices.

Firstly, we will develop the expression (4.76) for the different PEW basis functions. For this purpose, we have to take into consideration the normal components to $S_{1}^{(3)}$ of the $T E^{z}$ and $T M^{z}$ modes:

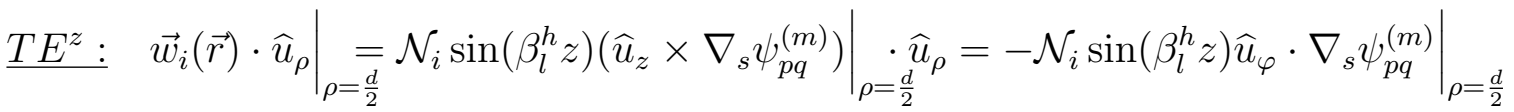

$$
\begin{aligned}
& =-\mathcal{N}_{i} p J_{p}\left(\chi_{p q}^{\prime}\right) \sin \left(\beta_{l}^{h} z\right) \frac{2}{d}\left\{\begin{array}{c}
-\sin (p \varphi) \\
\cos (p \varphi)
\end{array}\right\} \begin{array}{l}
(m)=(1) \\
(m)=(2)
\end{array}
\end{aligned}
$$

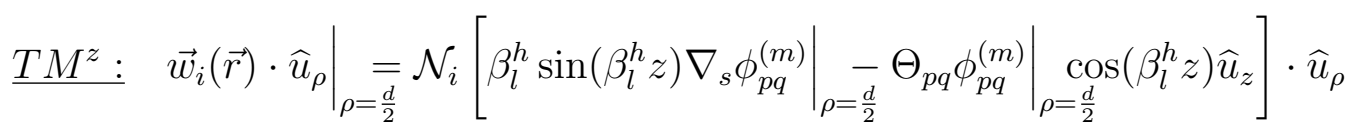

$$
\begin{aligned}
& =\mathcal{N}_{i} \beta_{l}^{h} \Theta_{p q} J_{p}^{\prime}\left(\chi_{p q}\right) \sin \left(\beta_{l}^{h} z\right)\left\{\begin{array}{l}
\cos (p \varphi) \\
\sin (p \varphi)
\end{array}\right\} \begin{array}{l}
(m)=(1) \\
(m)=(2)
\end{array}
\end{aligned}
$$

And the expression of $R$ in the cylindrical coordinate system:

$$
R=\sqrt{d^{2}\left(1-\cos \left(\varphi-\varphi^{\prime}\right)\right) / 2+\left(z-z^{\prime}\right)^{2}}
$$

By introducing now equations (4.77) and (4.78) in (4.76), we can obtain the value of the $\tilde{\mathbf{C}}^{(\mathbf{3 , 3})}$ entries for the different combination of basis functions. To achieve the results presented below, we use again the technique described in the Appendix B to reduce the integration over the $\varphi$ and $\varphi^{\prime}$ variables.

Case $\vec{w}_{i}$ is $T E^{z}$ and $\vec{w}_{j}$ is $T E^{z}:$

$$
\tilde{C}_{i j}^{(3,3)}=\frac{\mathcal{N}_{i} \mathcal{N}_{j} p u J_{p}\left(\chi_{p q}^{\prime}\right) J_{u}\left(\chi_{u v}^{\prime}\right)}{4 \pi} \delta_{m n} \delta_{p u} \tilde{I}_{p l, t}^{(3,3)}\left\{\begin{array}{l}
\left(1-\delta_{0 p}\right) \pi \\
\left(1+\delta_{0 p}\right) \pi
\end{array}\right\} \begin{aligned}
& (m)=(1) \\
& (m)=(2)
\end{aligned}
$$

Case $\vec{w}_{i}$ is $T E^{z}$ and $\vec{w}_{j}$ is $T M^{z}$ :

$$
\tilde{C}_{i j}^{(3,3)}=\frac{\mathcal{N}_{i} \mathcal{N}_{j} p \chi_{u v} J_{p}\left(\chi_{p q}^{\prime}\right) J_{u}^{\prime}\left(\chi_{u v}\right) \beta_{t}^{h}}{4 \pi}\left(1-\delta_{m n}\right) \delta_{p u} \tilde{I}_{p l, t}^{(3,3)}\left\{\begin{array}{c}
\left(1-\delta_{0 p}\right) \pi \\
-\left(1+\delta_{0 p}\right) \pi
\end{array}\right\} \begin{aligned}
& (m)=(1) \\
& (m)=(2)
\end{aligned}
$$


Case $\vec{w}_{i}$ is $T M^{z}$ and $\vec{w}_{j}$ is $T M^{z}$ :

$$
\tilde{C}_{i j}^{(3,3)}=\frac{\mathcal{N}_{i} \mathcal{N}_{j} \chi_{p q} \chi_{u v} J_{p}^{\prime}\left(\chi_{p q}\right) J_{u}^{\prime}\left(\chi_{u v}\right) \beta_{l}^{h} \beta_{t}^{h}}{4 \pi} \delta_{m n} \delta_{p u} \tilde{I}_{p l, t}^{(3,3)}\left\{\begin{array}{l}
\left(1+\delta_{0 p}\right) \pi \\
\left(1-\delta_{0 p}\right) \pi
\end{array}\right\} \begin{aligned}
& (m)=(1) \\
& (m)=(2)
\end{aligned}
$$

To obtain the expressions (4.79), (4.80) and (4.81), the following integral has been defined:

$$
\tilde{I}_{p l, t}^{(3,3)} \equiv \int_{0}^{h} d z \int_{0}^{h} d z^{\prime} \int_{0}^{2 \pi} d \varphi \frac{\sin \left(\beta_{l}^{h} z\right) \sin \left(\beta_{t}^{h} z^{\prime}\right) \cos (p \varphi)}{\sqrt{d^{2}(1-\cos \varphi) / 2+\left(z-z^{\prime}\right)^{2}}}
$$

Note that this integral is still singular when $R=0$, this is, when $z=z^{\prime}$ and $\varphi=0{ }^{7}$. For this reason, it can not be computed numerically in a direct way. It must be transformed analytically into regular integrals, as reported in detail in the Appendix D.

On the other hand, since $\tilde{\mathbf{C}}^{(\mathbf{3 , 3})}$ is a symmetric matrix, the case in which $\vec{w}_{i}$ is $T M^{z}$ and $\vec{w}_{j}$ is $T E^{z}$ is directly obtained interchanging the modal indexes $p, q, l,(m) \Leftrightarrow u, v, t,(n)$ from the case $\vec{w}_{i}$ is $T E^{z}$ and $\vec{w}_{j}$ is $T M^{z}$.

\section{Computation of matrix $\tilde{\mathbf{C}}^{(1,3)}$}

The entries of the $\tilde{\mathbf{C}}^{(\mathbf{1 , 3})}$ matrix are:

$$
\tilde{C}_{i j}^{(1,3)}=\int_{S_{1}^{(1)}} \int_{S_{1}^{(3)}}\left(-\left.\vec{w}_{i}(\vec{r})\right|_{z=0} ^{\cdot \widehat{u}_{z}}\right) \frac{1}{4 \pi R}\left(\left.\vec{w}_{j}\left(\vec{r}^{\prime}\right)\right|_{\substack{\rho^{\prime}=\frac{d}{2} \\ .}} ^{\cdot \widehat{u}_{\rho}^{\prime}}\right) d s d s^{\prime}
$$

Again, since the only subset of basis functions that have a non-zero normal component to the surface $S_{1}^{(1)}$ are the $T M^{z}$ modes, then

$$
\tilde{C}_{i j}^{(1,3)}=0 \quad \forall \vec{w}_{i} \in T E^{z}\{\vec{w}\}
$$

In this case, taking into account

$$
R=\sqrt{\rho^{2}+d^{2} / 4-\rho d \cos \left(\varphi-\varphi^{\prime}\right)+z^{\prime 2}}
$$

and introducing equations (4.62), (4.77) into (4.83), we can evaluate $\tilde{\mathbf{C}}^{(\mathbf{1}, \mathbf{3})}$ for the different combination of basis functions.

Case $\vec{w}_{i}$ is $T M^{z}$ and $\vec{w}_{j}$ is $T E^{z}$ :

$$
\tilde{C}_{i j}^{(1,3)}=\frac{\mathcal{N}_{i} \mathcal{N}_{j} \Theta_{p q}^{2} u J_{u}\left(\chi_{u v}^{\prime}\right)}{4 \pi}\left(1-\delta_{m n}\right) \delta_{p u} \tilde{I}_{p q, t}^{(1,3)}\left\{\begin{array}{l}
\left(1+\delta_{0 p}\right) \pi \\
\left(1-\delta_{0 p}\right) \pi
\end{array}\right\} \begin{aligned}
& (m)=(1) \\
& (m)=(2)
\end{aligned}
$$

\footnotetext{
${ }^{7}$ When $\vec{r}=\vec{r}^{\prime}$, we have $\varphi=0$ due to the change of variable performed by the technique described in the appendix to reduce the integrals over $\varphi$ and $\varphi^{\prime}$.
} 
Case $\vec{w}_{i}$ is $T M^{z}$ and $\vec{w}_{j}$ is $T M^{z}$ :

$$
\tilde{C}_{i j}^{(1,3)}=\frac{\mathcal{N}_{i} \mathcal{N}_{j} d \Theta_{p q}^{2} \Theta_{u v} \beta_{t}^{h} J_{u}^{\prime}\left(\chi_{u v}\right)}{8 \pi} \delta_{m n} \delta_{p u} \tilde{I}_{p q, t}^{(1,3)}\left\{\begin{array}{l}
\left(1+\delta_{0 p}\right) \pi \\
\left(1-\delta_{0 p}\right) \pi
\end{array}\right\} \begin{aligned}
& (m)=(1) \\
& (m)=(2)
\end{aligned}
$$

where the integral $\tilde{I}_{p q, t}^{(1,3)}$ has been defined as:

$$
\tilde{I}_{p q, t}^{(1,3)} \equiv \int_{0}^{\frac{d}{2}} d \rho \int_{0}^{h} d z^{\prime} \int_{0}^{2 \pi} d \varphi \frac{\rho J_{p}\left(\Theta_{p q} \rho\right) \sin \left(\beta_{t}^{h} z^{\prime}\right) \cos (p \varphi)}{\sqrt{\rho^{2}+d^{2} / 4-\rho d \cos (\varphi)+z^{\prime 2}}}
$$

It can be observed that when $R=0$, this is when $\rho=d / 2, \varphi=0$ and $z^{\prime}=0$, the integrand has an indeterminacy of the kind $0 / 0$. Due to this fact, we need to evaluate the existence of the limit of the integrand when $R \rightarrow 0$. As it is demonstrated in the Appendix $\mathrm{D}$, this limit exists and it is equal to zero, therefore we can integrate numerically $\tilde{I}_{p q, t}^{(1,3)}$.

$$
\lim _{\left\{\rho, \varphi, z^{\prime}\right\} \rightarrow\{d / 2,0,0\}} \frac{\rho J_{p}\left(\Theta_{p q} \rho\right) \sin \left(\beta_{t}^{h} z^{\prime}\right) \cos (p \varphi)}{\sqrt{\rho^{2}+d^{2} / 4-\rho d \cos (\varphi)+z^{\prime 2}}}=0
$$

\section{E. Computation of matrix $\tilde{\mathbf{C}}^{(2,3)}$}

The entries of the $\tilde{\mathbf{C}}^{(\mathbf{2 , 3})}$ matrix are:

$$
\tilde{C}_{i j}^{(2,3)}=\int_{S_{1}^{(2)}} \int_{S_{1}^{(3)}}\left(\vec{w}_{i}(\vec{r}) \mid \begin{array}{c}
\cdot \widehat{u}_{z} \\
z=h
\end{array}\right) \frac{1}{4 \pi R}\left(\left.\vec{w}_{j}\left(\vec{r}^{\prime}\right)\right|_{\substack{\cdot \\
\rho^{\prime}=\frac{d}{2}}} ^{\cdot \widehat{u}_{\rho}^{\prime}}\right) d s d s^{\prime}
$$

It can be easily observed that the only differences between the expressions (4.83) and (4.90) are the normal component of the basis functions to the surface $S_{1}^{(1)}$ and the surface $S_{1}^{(2)}$, and the dependence on $z^{\prime}$ of $R$. Due to this fact, it seems that we can take profit from the calculus performed for the $\tilde{\mathbf{C}}^{(\mathbf{1 , 3})}$ matrix in order to compute the $\tilde{\mathbf{C}}^{(\mathbf{2 , 3})}$ one.

Firstly, we must take into account that

$$
\left.\left(\vec{w}_{i}(\vec{r}) \cdot \widehat{n}^{(1)}\right)\right|_{z=0}=\left.(-1)^{l+1}\left(\vec{w}_{i}(\vec{r}) \cdot \widehat{n}^{(2)}\right)\right|_{z=h}
$$

Secondly, we must also consider the dependence on $z^{\prime}$ of $R$ which makes $\tilde{I}_{p q, t}^{(2,3)}$ to be:

$$
\tilde{I}_{p q, t}^{(2,3)} \equiv \int_{0}^{\frac{d}{2}} d \rho \int_{0}^{h} d z^{\prime} \int_{0}^{2 \pi} d \varphi \frac{\rho J_{p}\left(\Theta_{p q} \rho\right) \sin \left(\beta_{t}^{h} z^{\prime}\right) \cos (p \varphi)}{\sqrt{\rho^{2}+d^{2} / 4-\rho d \cos (\varphi)+\left(h-z^{\prime}\right)^{2}}}
$$


By performing a change of variable $u=h-z^{\prime}$, we have

$$
\begin{aligned}
\tilde{I}_{p q, t}^{(2,3)} & =-\int_{0}^{\frac{d}{2}} d \rho \int_{0}^{2 \pi} d \varphi \int_{h}^{0} d u \frac{\rho J_{p}\left(\Theta_{p q} \rho\right) \sin \left(\beta_{t}^{h}(h-u)\right) \cos (p \varphi)}{\sqrt{\rho^{2}+d^{2} / 4-\rho d \cos (\varphi)+u^{2}}} \\
& =(-1)^{t+1} \int_{0}^{\frac{d}{2}} d \rho \int_{0}^{2 \pi} d \varphi \int_{0}^{h} d u \frac{\rho J_{p}\left(\Theta_{p q} \rho\right) \sin \left(\beta_{t}^{h} u\right) \cos (p \varphi)}{\sqrt{\rho^{2}+d^{2} / 4-\rho d \cos (\varphi)+u^{2}}}=(-1)^{t+1} \tilde{I}_{p q, t}^{(1,3)}
\end{aligned}
$$

Therefore, using equations (4.91) and (4.93) in (4.90), we obtain:

$$
\tilde{C}_{i j}^{(2,3)}=(-1)^{(l+t)} \tilde{C}_{i j}^{(1,3)}
$$

\subsubsection{Computation of the $L$ matrix}

The $\mathbf{L}$ matrix is a real matrix whose entries are described by:

$$
L_{i j}=\int_{V_{1}} \int_{V_{1}} \vec{w}_{i}(\vec{r}) \cdot \underline{G}_{o}^{A}\left(\vec{r}, \vec{r}^{\prime}\right) \cdot \vec{w}_{j}\left(\vec{r}^{\prime}\right) d v d v^{\prime}
$$

where $V_{1}$ is the cylindrical volume defined by the DR; $\vec{w}_{i}(\vec{r})$ and $\vec{w}_{j}\left(\vec{r}^{\prime}\right)$ are the basis functions; $i, j=1,2, \ldots, Q$, being $Q$ the number of basis functions used to expand the polarization vector by the method of moments; $\underline{G}_{o}^{A}\left(\vec{r}, \vec{r}^{\prime}\right)$ is the quasi-static dyadic Green's function for the electric vector potential. It is easy to demonstrate that it is a symmetric matrix, since:

$$
\begin{aligned}
L_{j i} & =\int_{V_{1}} \int_{V_{1}} \vec{w}_{j}(\vec{r}) \cdot \underline{G}_{o}^{A}\left(\vec{r}, \vec{r}^{\prime}\right) \cdot \vec{w}_{i}\left(\vec{r}^{\prime}\right) d v d v^{\prime}=\int_{V_{1}} \int_{V_{1}} \vec{w}_{j}(\vec{r}) \cdot\left(\vec{w}_{i}\left(\vec{r}^{\prime}\right) \cdot\left[\underline{G}_{o}^{A}\left(\vec{r}, \vec{r}^{\prime}\right)\right]_{T}\right) d v d v^{\prime} \\
& =\int_{V_{1}} \int_{V_{1}} \vec{w}_{i}\left(\vec{r}^{\prime}\right) \cdot \underline{G}_{o}^{A}\left(\vec{r}^{\prime}, \vec{r}\right) \cdot \vec{w}_{j}(\vec{r}) d v d v^{\prime}=L_{i j}
\end{aligned}
$$

where we have used the property $\left[\underline{G}_{o}^{A}\left(\vec{r}, \vec{r}^{\prime}\right)\right]_{T}=\underline{G}_{o}^{A}\left(\vec{r}^{\prime}, \vec{r}\right)$.

As it has been shown in section 2.6, this dyadic contains the singularity of the dyadic Green's function for the electric potential $\underline{G}^{A}$. In fact, by using equation (2.82) we can extract the singularity in closed form.

$$
\underline{G}_{o}^{A}\left(\vec{r}, \vec{r}^{\prime}\right)=\underline{G}_{s}^{o}(\vec{R})+\underline{G}_{o, r}^{A}\left(\vec{r}, \vec{r}^{\prime}\right)=\frac{1}{8 \pi R}\left(\underline{I}+\frac{\vec{R} \vec{R}}{R^{2}}\right)+\underline{G}_{o, r}^{A}\left(\vec{r}, \vec{r}^{\prime}\right)
$$

where $\vec{R}=\vec{r}-\vec{r}^{\prime}$, and $\underline{G}_{o, r}^{A}\left(\vec{r}, \vec{r}^{\prime}\right)$ is the regular part of the quasi-static dyadic Green's function.

By introducing equation (4.97) into (4.96), the $\mathbf{L}$ matrix can be expressed as the sum of two matrices: the former one containing the singularity of the Green's function, and the last one containing the regular part. Thus, we can write:

$$
\mathbf{L}=\mathbf{L}^{\text {sing }}+\mathbf{L}^{\text {reg }}
$$


where the entries of the $\mathbf{L}^{\text {sing }}$ and $\mathbf{L}^{\text {reg }}$ are defined by:

$$
\begin{gathered}
L_{i j}^{\text {sing }} \equiv \int_{V_{1}} \int_{V_{1}} \vec{w}_{i}(\vec{r}) \cdot \underline{G}_{s}^{o}(\vec{R}) \cdot \vec{w}_{j}\left(\vec{r}^{\prime}\right) d v d v^{\prime} \\
L_{i j}^{r e g} \equiv \int_{V_{1}} \int_{V_{1}} \vec{w}_{i}(\vec{r}) \cdot \underline{G}_{o, r}^{A}\left(\vec{r}, \vec{r}^{\prime}\right) \cdot \vec{w}_{j}\left(\vec{r}^{\prime}\right) d v d v^{\prime}
\end{gathered}
$$

Since $\underline{G}_{s}^{o}(\vec{R})$ is a singular function when $\vec{r}=\vec{r}^{\prime}$, we need to transform the singular integral in (4.99a) into a regular one in order to calculate it. On the other hand, the integrand in (4.99b) is a regular function, and thus, by using the Ewald's technique to compute the dyadic $G_{o, r}^{A}$, we have computed the integral numerically. However, it must be observed that expressions (4.99) involve volume-volume integrals (6-D integrals) which require a big computational effort, reducing significantly the efficiency of the algorithm implemented. Due to this fact, in this case, it is necessary to reformulate the volume-volume integrals to surface-surface ones. In this way, we only need to perform a 2-D mesh over the surface of the dielectric resonator, instead of performing a 3-D mesh in its volume. These transformations are specially important for the evaluation of the regular part of the $\mathbf{L}$ matrix, since they allow to drastically reduce the required computational effort.

\section{A. Computation of the $L^{\text {sing }}$ matrix}

The entries of the $\mathbf{L}^{\text {sing }}$ matrix are defined by the following integral:

$$
I=\int_{V_{1}} \int_{V_{1}} \vec{w}_{i}(\vec{r}) \cdot \underline{G}_{s}^{o}(\vec{R}) \cdot \vec{w}_{j}\left(\vec{r}^{\prime}\right) d v d v^{\prime}
$$

By making use of the identity (4.1) and applying the second vector-dyadic Green's theorem (eq. (C.12) of Appendix C), we have:

$$
\begin{aligned}
& k_{i}^{2} \int_{V_{1}} \vec{w}_{i}(\vec{r}) \cdot \underline{G}_{s}^{o}(\vec{R}) d v=\int_{V_{1}}\left(\nabla \times \nabla \times \vec{w}_{i}(\vec{r})\right) \cdot \underline{G}_{s}^{o}(\vec{R}) d v \\
& =\int_{V_{1}} \vec{w}_{i}(\vec{r}) \cdot \nabla \times \nabla \times \underline{G}_{s}^{o}(\vec{R}) d v+ \\
& \quad+\int_{S_{1}}\left(\widehat{n} \times \vec{w}_{i}(\vec{r})\right) \cdot \nabla \times \underline{G}_{s}^{o}(\vec{R}) d s+\int_{S_{1}}\left(\widehat{n} \times \nabla \times \vec{w}_{i}(\vec{r})\right) \cdot \underline{G}_{s}^{o}(\vec{R}) d s
\end{aligned}
$$

and thus, introducing this expression into (4.100), we can write:

$$
\begin{aligned}
k_{i}^{2} I= & \int_{V_{1}} \int_{V_{1}} \vec{w}_{i}(\vec{r}) \cdot \nabla \times \nabla \times \underline{G}_{s}^{o}(\vec{R}) \cdot \vec{w}_{j}\left(\vec{r}^{\prime}\right) d v d v^{\prime} \\
& +\int_{V_{1}} \int_{S_{1}}\left(\widehat{n} \times \vec{w}_{i}(\vec{r})\right) \cdot \nabla \times \underline{G}_{s}^{o}(\vec{R}) \cdot \vec{w}_{j}\left(\vec{r}^{\prime}\right) d s d v^{\prime} \\
& +\int_{V_{1}} \int_{S_{1}}\left(\widehat{n} \times \nabla \times \vec{w}_{i}(\vec{r})\right) \cdot \underline{G}_{s}^{o}(\vec{R}) \cdot \vec{w}_{j}\left(\vec{r}^{\prime}\right) d s d v^{\prime}
\end{aligned}
$$


Next, taking into account the identity satisfied by the dyadic $\underline{G}_{s}^{o}(\vec{R})$ (see section E.1 of Appendix E),

$$
\nabla \times \nabla \times \underline{G}_{s}^{o}(\vec{R})=\nabla^{\prime} \times \nabla^{\prime} \times \underline{G}_{s}^{o}(\vec{R})
$$

and using the expression (C.20), we can develop the double volume integral in (4.102) to obtain:

$$
\begin{aligned}
& \int_{V_{1}} \nabla \times \nabla \times \underline{G}_{s}^{o}(\vec{R}) \cdot \vec{w}_{j}\left(\vec{r}^{\prime}\right) d v^{\prime}=\int_{V_{1}} \nabla^{\prime} \times \nabla^{\prime} \times \underline{G}_{s}^{o}(\vec{R}) \cdot \vec{w}_{j}\left(\vec{r}^{\prime}\right) d v^{\prime} \\
& =k_{j}^{2} \int_{V_{1}} \underline{G}_{s}^{o}(\vec{R}) \cdot \vec{w}_{j}\left(\vec{r}^{\prime}\right) d v^{\prime}-\int_{S_{1}}\left(\nabla^{\prime} \times \underline{G}_{s}^{o}(\vec{R})\right) \cdot\left(\widehat{n}^{\prime} \times \vec{w}_{j}\left(\vec{r}^{\prime}\right)\right) d s^{\prime} \\
& -\int_{S_{1}} \underline{G}_{s}^{o}(\vec{R}) \cdot\left(\hat{n}^{\prime} \times \nabla^{\prime} \times \vec{w}_{j}\left(\vec{r}^{\prime}\right)\right) d s^{\prime}
\end{aligned}
$$

According to this last expression, we can rewrite equation (4.102),

$$
\begin{aligned}
k_{i}^{2} I=k_{j}^{2} & \int_{V_{1}} \int_{V_{1}} \vec{w}_{i}(\vec{r}) \cdot \underline{G}_{s}^{o}(\vec{R}) \cdot \vec{w}_{j}\left(\vec{r}^{\prime}\right) d v d v^{\prime} \\
& -\int_{V_{1}} \int_{S_{1}} \vec{w}_{i}(\vec{r}) \cdot\left(\nabla^{\prime} \times \underline{G}_{s}^{o}(\vec{R})\right) \cdot\left(\widehat{n}^{\prime} \times \vec{w}_{j}\left(\vec{r}^{\prime}\right)\right) d s^{\prime} d v \\
& -\int_{V_{1}} \int_{S_{1}} \vec{w}_{i}(\vec{r}) \cdot \underline{G}_{s}^{o}(\vec{R}) \cdot\left(\widehat{n}^{\prime} \times \nabla^{\prime} \times \vec{w}_{j}\left(\vec{r}^{\prime}\right)\right) d s^{\prime} d v \\
& +\int_{V_{1}} \int_{S_{1}}\left(\widehat{n} \times \vec{w}_{i}(\vec{r})\right) \cdot \nabla \times \underline{G}_{s}^{o}(\vec{R}) \cdot \vec{w}_{j}\left(\vec{r}^{\prime}\right) d s d v^{\prime} \\
& +\int_{V_{1}} \int_{S_{1}}\left(\widehat{n} \times \nabla \times \vec{w}_{i}(\vec{r})\right) \cdot \underline{G}_{s}^{o}(\vec{R}) \cdot \vec{w}_{j}\left(\vec{r}^{\prime}\right) d s d v^{\prime},
\end{aligned}
$$

and making use of the property(see section E.1 of Appendix E):

$$
\nabla \times \underline{G}_{s}^{o}(\vec{R})=\nabla^{\prime} \times \underline{G}_{s}^{o}(\vec{R})
$$

we finally have:

$$
\begin{aligned}
\left(k_{i}^{2}-k_{j}^{2}\right) I= & -\int_{V_{1}} \int_{S_{1}} \vec{w}_{i}(\vec{r}) \cdot\left(\nabla \times \underline{G}_{s}^{o}(\vec{R})\right) \cdot\left(\widehat{n}^{\prime} \times \vec{w}_{j}\left(\vec{r}^{\prime}\right)\right) d s^{\prime} d v \\
& -\int_{V_{1}} \int_{S_{1}} \vec{w}_{i}(\vec{r}) \cdot \underline{G}_{s}^{o}(\vec{R}) \cdot\left(\widehat{n}^{\prime} \times \nabla^{\prime} \times \vec{w}_{j}\left(\vec{r}^{\prime}\right)\right) d s^{\prime} d v \\
& +\int_{V_{1}} \int_{S_{1}}\left(\widehat{n} \times \vec{w}_{i}(\vec{r})\right) \cdot \nabla^{\prime} \times \underline{G}_{s}^{o}(\vec{R}) \cdot \vec{w}_{j}\left(\vec{r}^{\prime}\right) d s d v^{\prime} \\
& +\int_{V_{1}} \int_{S_{1}}\left(\widehat{n} \times \nabla \times \vec{w}_{i}(\vec{r})\right) \cdot \underline{G}_{s}^{o}(\vec{R}) \cdot \vec{w}_{j}\left(\vec{r}^{\prime}\right) d s d v^{\prime}
\end{aligned}
$$


The result achieved so far allows to calculate the entries of the $\mathbf{L}^{\text {sing }}$ matrix in terms of 5-D integrals instead of 6-D ones, when $k_{i} \neq k_{j}$. Nevertheless, the integrands in (4.107) are still singular when $R=0$, so we need to transform them once again in order to obtain regular integrals.

For this purpose, let us consider the following properties of the singular dyadic (see section E.1 of Appendix E):

$$
\begin{gathered}
\underline{G}_{s}^{o}(\vec{R})=-\nabla \times \nabla \times \frac{R \underline{I}}{8 \pi}=-\nabla^{\prime} \times \nabla^{\prime} \times \frac{R \underline{I}}{8 \pi} \\
\nabla \times \underline{G}_{s}^{o}(\vec{R})=-\nabla \times \nabla \times \frac{\vec{R} \times \underline{I}}{8 \pi R} \\
\nabla^{\prime} \times \underline{G}_{s}^{o}(\vec{R})=-\nabla^{\prime} \times \nabla^{\prime} \times \frac{\vec{R} \times \underline{I}}{8 \pi R}
\end{gathered}
$$

In order to transform the volume integrals contained in equation (4.107), we use (C.12) and (4.108) for the integrals over $\vec{r}$, so that

$$
\begin{aligned}
\int_{V_{1}} \vec{w}_{i}(\vec{r}) \cdot \nabla \times \underline{G}_{s}^{o}(\vec{R}) d v= & -k_{i}^{2} \int_{V_{1}} \vec{w}_{i}(\vec{r}) \cdot \frac{\vec{R} \times \underline{I}}{8 \pi R} d v+\int_{S_{1}}\left(\widehat{n} \times \nabla \times \vec{w}_{i}(\vec{r})\right) \cdot \frac{\vec{R} \times \underline{I}}{8 \pi R} d s \\
& -\int_{S_{1}}\left(\widehat{n} \times \vec{w}_{i}(\vec{r})\right) \cdot \underline{G}_{s}^{o}(\vec{R}) d s \\
\int_{V_{1}} \vec{w}_{i}(\vec{r}) \cdot \underline{G}_{s}^{o}(\vec{R}) d v= & -k_{i}^{2} \int_{V_{1}} \vec{w}_{i}(\vec{r}) \frac{R}{8 \pi} d v+\int_{S_{1}}\left(\widehat{n} \times \nabla \times \vec{w}_{i}(\vec{r})\right) \frac{R}{8 \pi} d s \\
& +\int_{S_{1}}\left(\widehat{n} \times \vec{w}_{i}(\vec{r})\right) \cdot \frac{\vec{R} \times \underline{I}}{8 \pi R} d s
\end{aligned}
$$

Analogously, we use equation (C.20) to develop the integrals over $\vec{r}^{\prime}$, obtaining:

$$
\begin{aligned}
\int_{V_{1}}\left(\nabla^{\prime} \times \underline{G}_{s}^{o}(\vec{R})\right) \cdot \vec{w}_{j}\left(\vec{r}^{\prime}\right) d v^{\prime}= & -k_{j}^{2} \int_{V_{1}} \frac{\vec{R} \times \underline{I}}{8 \pi R} \cdot \vec{w}_{j}\left(\vec{r}^{\prime}\right) d v^{\prime}-\int_{S_{1}} G_{s}^{o}(\vec{R}) \cdot\left(\vec{n}^{\prime} \times \vec{w}_{j}\left(\vec{r}^{\prime}\right)\right) d s^{\prime} \\
& +\int_{S_{1}} \frac{\vec{R} \times \underline{I}}{8 \pi R} \cdot\left(\widehat{n}^{\prime} \times \nabla^{\prime} \times \vec{w}_{j}\left(\vec{r}^{\prime}\right)\right) d s^{\prime} \\
\int_{V_{1}} \underline{G}_{s}^{o}(\vec{R}) \cdot \vec{w}_{j}\left(\vec{r}^{\prime}\right) d v^{\prime}= & -k_{j}^{2} \int_{V_{1}} \frac{R}{8 \pi} \vec{w}_{j}\left(\vec{r}^{\prime}\right) d v^{\prime}+\int_{S_{1}} \frac{\vec{R} \times \underline{I}}{8 \pi R} \cdot\left(\widehat{n}^{\prime} \times \vec{w}_{j}\left(\vec{r}^{\prime}\right)\right) d s^{\prime} \\
& +\int_{S_{1}} \frac{R}{8 \pi}\left(\widehat{n}^{\prime} \times \nabla^{\prime} \times \vec{w}_{j}\left(\vec{r}^{\prime}\right)\right) d s^{\prime}
\end{aligned}
$$


We can now introduce (4.109), (4.110), (4.111) and (4.112) into equation (4.107), obtaining:

$$
\begin{aligned}
\left(k_{i}^{2}-k_{j}^{2}\right) I= & k_{i}^{2} \int_{S_{1}} \int_{V_{1}} \vec{w}_{i}(\vec{r}) \cdot \frac{\vec{R}}{8 \pi R} \times\left(\widehat{n}^{\prime} \times \vec{w}_{j}\left(\vec{r}^{\prime}\right)\right) d v d s^{\prime} \\
& -\int_{S_{1}} \int_{S_{1}}\left(\widehat{n} \times \nabla \times \vec{w}_{i}(\vec{r})\right) \cdot \frac{\vec{R}}{8 \pi R} \times\left(\widehat{n}^{\prime} \times \vec{w}_{j}\left(\vec{r}^{\prime}\right)\right) d s d s^{\prime} \\
& +\int_{S_{1}} \int_{S_{1}}\left(\widehat{n} \times \vec{w}_{i}(\vec{r})\right) \cdot \underline{G}_{s}^{o}(\vec{R}) \cdot\left(\widehat{n}^{\prime} \times \vec{w}_{j}\left(\vec{r}^{\prime}\right)\right) d s d s^{\prime} \\
& +k_{i}^{2} \int_{S_{1}} \int_{V_{1}} \vec{w}_{i}(\vec{r}) \frac{R}{8 \pi} \cdot\left(\widehat{n}^{\prime} \times \nabla^{\prime} \times \vec{w}_{j}\left(\vec{r}^{\prime}\right)\right) d v d s^{\prime} \\
& -\int_{S_{1}} \int_{S_{1}}\left(\widehat{n} \times \nabla \times \vec{w}_{i}(\vec{r})\right) \frac{R}{8 \pi} \cdot\left(\widehat{n}^{\prime} \times \nabla^{\prime} \times \vec{w}_{j}\left(\vec{r}^{\prime}\right)\right) d s d s^{\prime} \\
& -\int_{S_{1}} \int_{S_{1}}\left(\widehat{n} \times \vec{w}_{i}(\vec{r})\right) \cdot \frac{\vec{R}}{8 \pi R} \times\left(\widehat{n}^{\prime} \times \nabla^{\prime} \times \vec{w}_{j}\left(\vec{r}^{\prime}\right)\right) d s d s^{\prime} \\
& -k_{j}^{2} \int_{S_{1}} \int_{V_{1}}\left(\widehat{n} \times \vec{w}_{i}(\vec{r})\right) \cdot \frac{\vec{R}}{8 \pi R} \times \vec{w}_{j}\left(\vec{r}^{\prime}\right) d v^{\prime} d s \\
& -\int_{S_{1}} \int_{S_{1}}\left(\widehat{n} \times \vec{w}_{i}(\vec{r})\right) \cdot \underline{G_{s}^{o}}(\vec{R}) \cdot\left(\widehat{n}^{\prime} \times \vec{w}_{j}\left(\vec{r}^{\prime}\right)\right) d s^{\prime} d s \\
& +\int_{S_{1}} \int_{S_{1}}\left(\widehat{n} \times \vec{w}_{i}(\vec{r})\right) \cdot \frac{\vec{R}}{8 \pi R} \times\left(\widehat{n}^{\prime} \times \nabla^{\prime} \times \vec{w}_{j}\left(\vec{r}^{\prime}\right)\right) d s^{\prime} d s \\
& -k_{j}^{2} \int_{S_{1}} \int_{V_{1}}\left(\widehat{n} \times \nabla \times \vec{w}_{i}(\vec{r})\right) \frac{R}{8 \pi} \cdot \vec{w}_{j}\left(\vec{r}^{\prime}\right) d v^{\prime} d s \\
& +\int_{S_{1}} \int_{S_{1}}\left(\widehat{n} \times \nabla \times \vec{w}_{i}(\vec{r})\right) \cdot \frac{\vec{R}}{8 \pi R} \times\left(\widehat{n}^{\prime} \times \vec{w}_{j}\left(\vec{r}^{\prime}\right)\right) d s^{\prime} d s \\
& +\int_{S_{1}} \int_{S_{1}}\left(\widehat{n} \times \nabla \times \vec{w}_{i}(\vec{r})\right) \frac{R}{8 \pi} \cdot\left(\widehat{n}^{\prime} \times \nabla^{\prime} \times \vec{w}_{j}\left(\vec{r}^{\prime}\right)\right) d s^{\prime} d s
\end{aligned}
$$

Simplifying this expression, we have:

$$
\begin{aligned}
\left(k_{i}^{2}-k_{j}^{2}\right) I & =k_{i}^{2} \int_{S_{1}} \int_{V_{1}} \vec{w}_{i}(\vec{r}) \cdot \frac{\vec{R}}{8 \pi R} \times\left(\widehat{n}^{\prime} \times \vec{w}_{j}\left(\vec{r}^{\prime}\right)\right) d v d s^{\prime} \\
& +k_{i}^{2} \int_{S_{1}} \int_{V_{1}} \vec{w}_{i}(\vec{r}) \frac{R}{8 \pi} \cdot\left(\widehat{n}^{\prime} \times \nabla^{\prime} \times \vec{w}_{j}\left(\vec{r}^{\prime}\right)\right) d v d s^{\prime} \\
& -k_{j}^{2} \int_{S_{1}} \int_{V_{1}}\left(\widehat{n} \times \vec{w}_{i}(\vec{r})\right) \cdot \frac{\vec{R}}{8 \pi R} \times \vec{w}_{j}\left(\vec{r}^{\prime}\right) d v^{\prime} d s \\
& -k_{j}^{2} \int_{S_{1}} \int_{V_{1}}\left(\widehat{n} \times \nabla \times \vec{w}_{i}(\vec{r})\right) \frac{R}{8 \pi} \cdot \vec{w}_{j}\left(\vec{r}^{\prime}\right) d v^{\prime} d s
\end{aligned}
$$


Hence, this expression permits to calculate the entries of the singular $\mathbf{L}$ matrix, when $k_{i} \neq k_{j}$, in terms of 5-D integrals of regular functions. However, we can further transform these integrals using the following expressions:

$$
\begin{aligned}
\int_{V_{1}} \vec{w}_{i}(\vec{r}) \times \frac{\vec{R}}{R} d v & =\int_{V_{1}} \vec{w}_{i}(\vec{r}) \times \nabla \vec{R} d v \\
& =-\int_{V_{1}} \nabla \times\left(R \vec{w}_{i}(\vec{r})\right) d v+\int_{V_{1}} R \nabla \times \vec{w}_{i}(\vec{r}) d v \\
& =-\int_{S_{1}} \widehat{n} \times \vec{w}_{i}(\vec{r}) R d s+\int_{V_{1}} R \nabla \times \vec{w}_{i}(\vec{r}) d v
\end{aligned}
$$

and,

$$
\begin{aligned}
-\int_{V_{1}} \frac{\vec{R}}{R} \times \vec{w}_{j}\left(\vec{r}^{\prime}\right) d v^{\prime} & =\int_{V_{1}}\left(\nabla^{\prime} R\right) \times \vec{w}_{j}\left(\vec{r}^{\prime}\right) d v^{\prime} \\
& =\int_{V_{1}} \nabla^{\prime} \times\left(R \vec{w}_{j}\left(\vec{r}^{\prime}\right)\right) d v^{\prime}-\int_{V_{1}} R \nabla^{\prime} \times \vec{w}_{j}\left(\vec{r}^{\prime}\right) d v^{\prime} \\
& =\int_{S_{1}} \widehat{n}^{\prime} \times \vec{w}_{j}\left(\vec{r}^{\prime}\right) R d s^{\prime}-\int_{V_{1}} R \nabla^{\prime} \times \vec{w}_{j}\left(\vec{r}^{\prime}\right) d v^{\prime}
\end{aligned}
$$

Finally, substituting these results into equation (4.114), we obtain:

$$
\begin{aligned}
\left(k_{i}^{2}-k_{j}^{2}\right) L_{i j}^{s i n g} & =k_{i}^{2} \int_{S_{1}} \int_{V_{1}} \frac{R}{8 \pi} \nabla \times \vec{w}_{i}(\vec{r}) \cdot\left(\widehat{n}^{\prime} \times \vec{w}_{j}\left(\vec{r}^{\prime}\right)\right) d v d s^{\prime} \\
& +k_{i}^{2} \int_{S_{1}} \int_{V_{1}} \frac{R}{8 \pi} \vec{w}_{i}(\vec{r}) \cdot\left(\widehat{n}^{\prime} \times \nabla^{\prime} \times \vec{w}_{j}\left(\vec{r}^{\prime}\right)\right) d v d s^{\prime} \\
& -k_{j}^{2} \int_{S_{1}} \int_{V_{1}} \frac{R}{8 \pi}\left(\widehat{n} \times \vec{w}_{i}(\vec{r})\right) \cdot \nabla^{\prime} \times \vec{w}_{j}\left(\vec{r}^{\prime}\right) d v^{\prime} d s \\
& -k_{j}^{2} \int_{S_{1}} \int_{V_{1}} \frac{R}{8 \pi}\left(\widehat{n} \times \nabla \times \vec{w}_{i}(\vec{r})\right) \cdot \vec{w}_{j}\left(\vec{r}^{\prime}\right) d v^{\prime} d s \\
& -\left(k_{i}^{2}-k_{j}^{2}\right) \int_{S_{1}} \int_{S_{1}} \frac{R}{8 \pi}\left(\widehat{n} \times \vec{w}_{i}(\vec{r})\right) \cdot\left(\hat{n}^{\prime} \times \vec{w}_{j}\left(\vec{r}^{\prime}\right)\right) d s d s^{\prime}
\end{aligned}
$$

Expression (4.117) is numerically more stable than expression (4.114), since there are no denominators approaching to zero in the involved integrands. This result provides directly $L_{i j}^{s i n g}$ as a five-dimension integral of regular functions, when $k_{i} \neq k_{j}$, whereas it yields a 
relationship independent of $L_{i j}^{\text {sing }}$ when $k_{i}=k_{j}$.

To deal with this particular case, we can introduce (4.110) into (4.99a) to obtain:

$$
\begin{aligned}
L_{i j}^{\text {sing }} & =\int_{V_{1}} \int_{V_{1}} \vec{w}_{i}(\vec{r}) \cdot \underline{G}_{s}^{o}(\vec{R}) \cdot \vec{w}_{j}\left(\vec{r}^{\prime}\right) d v d v^{\prime} \\
& =-k_{i}^{2} \int_{V_{1}} \int_{V_{1}} \vec{w}_{i}(\vec{r}) \frac{R}{8 \pi} \cdot \vec{w}_{j}\left(\vec{r}^{\prime}\right) d v d v^{\prime}+\int_{V_{1}} \int_{S_{1}}\left(\widehat{n} \times \nabla \times \vec{w}_{i}(\vec{r})\right) \frac{R}{8 \pi} \cdot \vec{w}_{j}\left(\vec{r}^{\prime}\right) d s d v^{\prime} \\
& +\int_{V_{1}} \int_{S_{1}}\left(\widehat{n} \times \vec{w}_{i}(\vec{r})\right) \cdot \frac{\vec{R}}{8 \pi R} \times \vec{w}_{j}\left(\vec{r}^{\prime}\right) d s d v^{\prime}
\end{aligned}
$$

and making use of the expression (4.116), we obtain:

$$
\begin{aligned}
L_{i j}^{\text {sing }}= & -k_{i}^{2} \int_{V_{1}} \int_{V_{1}} \frac{R}{8 \pi} \vec{w}_{i}(\vec{r}) \cdot \vec{w}_{j}\left(\vec{r}^{\prime}\right) d v d v^{\prime}+\int_{V_{1}} \int_{S_{1}} \frac{R}{8 \pi}\left(\widehat{n} \times \nabla \times \vec{w}_{i}(\vec{r})\right) \cdot \vec{w}_{j}\left(\vec{r}^{\prime}\right) d s d v^{\prime} \\
& +\int_{V_{1}} \int_{S_{1}} \frac{R}{8 \pi}\left(\widehat{n} \times \vec{w}_{i}(\vec{r})\right) \cdot\left(\nabla^{\prime} \times \vec{w}_{j}\left(\vec{r}^{\prime}\right)\right) d s d v^{\prime} \\
& -\int_{S_{1}} \int_{S_{1}} \frac{R}{8 \pi}\left(\widehat{n} \times \vec{w}_{i}(\vec{r})\right) \cdot\left(\widehat{n}^{\prime} \times \vec{w}_{j}\left(\vec{r}^{\prime}\right)\right) d s d s^{\prime}
\end{aligned}
$$

The numerical computation of all previous integrals has been performed in the cylindrical coordinate system centered in the bottom base of the dielectric resonator, where the basis functions used in the Method of Moments have been defined. In fact, due to the dependency of these regular functions with the angular variables $\varphi$ and $\varphi^{\prime}$, all the integrals presented above can be reduced in one more dimension, and even a great number of them are directly equal to zero (see Appendix B). Therefore, the integrals involved in the calculation of $\mathbf{L}^{\text {sing }}$ have been reduced to $4-\mathrm{D}$ regular integrals when $k_{i} \neq k_{j}$ case. On the other hand, when $k_{i}=k_{j}, 5-\mathrm{D}$ regular integrals need to be computed, but this is not a big drawback since they represent a very reduced percentage of the total number of integrals to be computed. However, there is an alternative procedure to compute $\mathbf{L}^{\text {sing }}$ when $k_{i}=k_{j}$ that results in 4-D integrals.

It can be observed from equation (4.117) that, when $k_{i}=k_{j}$, the expression of $L_{i j}^{\text {sing }}$ is an indeterminate form of the type $0 / 0$ that could be calculated as the limit for $k_{i} \rightarrow k_{j}$, if $k_{i}$ would be a continuous independent variable (but this is not the case, due to the boundary conditions imposed to $\left.\vec{w}_{i}\right)$. Let us consider the function $\vec{w}_{i}(\alpha \vec{r})$, defined in $V_{1}$, where $\alpha$ is a continuous scaling factor. Obviously, $\vec{w}_{i}(\alpha \vec{r})$ does not satisfy the same boundary conditions on $S_{V_{1}}$ (unless for $\alpha=1$ ), but in $V_{1}$ it still satisfies:

$$
\nabla \cdot \vec{w}_{i}(\alpha \vec{r})=0, \quad \nabla \times \nabla \times \vec{w}_{i}(\alpha \vec{r})=\alpha^{2} k_{i}^{2} \vec{w}_{i}(\alpha \vec{r})=\alpha^{2} k_{j}^{2} \vec{w}_{i}(\alpha \vec{r}),
$$

and the limit,

$$
\lim _{\alpha \rightarrow 1} \vec{w}_{i}(\alpha \vec{r})=\vec{w}_{i}(\vec{r})
$$


For this reason, the result above (equation (4.117)) applied to $\vec{w}_{i}(\alpha \vec{r})$ provides meaningful results for any $\alpha \neq 1$, even in the $k_{i}=k_{j}$ case. Nevertheless, taking the limit for $\alpha \rightarrow 1$, we can write:

$$
I=\int_{V_{1}} \int_{V_{1}} \vec{w}_{i}(\vec{r}) \cdot \underline{G}_{s}^{o}(\vec{R}) \cdot \vec{w}_{j}\left(\vec{r}^{\prime}\right) d v d v^{\prime}=\lim _{\alpha \rightarrow 1} \int_{V_{1}} \int_{V_{1}} \vec{w}_{i}(\alpha \vec{r}) \cdot \underline{G}_{s}^{o}(\vec{R}) \cdot \vec{w}_{j}\left(\vec{r}^{\prime}\right) d v d v^{\prime}
$$

And thus, introducing (4.117) in (4.121) for $\vec{w}_{i}(\alpha \vec{r})$, and making use of (4.120), we may rewrite the integral when $k_{i}=k_{j}$ as follows:

$$
\begin{aligned}
I=\lim _{\alpha \rightarrow 1}\left\{\frac{1}{\alpha^{2}-1}(\right. & \alpha^{2} \int_{S_{1}} \int_{V_{1}} \frac{R}{8 \pi} \nabla \times \vec{w}_{i}(\alpha \vec{r}) \cdot\left(\widehat{n}^{\prime} \times \vec{w}_{j}\left(\vec{r}^{\prime}\right)\right) d v d s^{\prime} \\
& +\alpha^{2} \int_{S_{1}} \int_{V_{1}} \frac{R}{8 \pi} \vec{w}_{i}(\alpha \vec{r}) \cdot\left(\widehat{n}^{\prime} \times \nabla^{\prime} \times \vec{w}_{j}\left(\vec{r}^{\prime}\right)\right) d v d s^{\prime} \\
& -\int_{S_{1}} \int_{V_{1}} \frac{R}{8 \pi}\left(\widehat{n} \times \vec{w}_{i}(\alpha \vec{r})\right) \cdot \nabla^{\prime} \times \vec{w}_{j}\left(\vec{r}^{\prime}\right) d v^{\prime} d s \\
& \left.-\int_{S_{1}} \int_{V_{1}} \frac{R}{8 \pi}\left(\widehat{n} \times \nabla \times \vec{w}_{i}(\alpha \vec{r})\right) \cdot \vec{w}_{j}\left(\vec{r}^{\prime}\right) d v^{\prime} d s\right) \\
& \left.-\int_{S_{1}} \int_{S_{1}} \frac{R}{8 \pi}\left(\widehat{n} \times \vec{w}_{i}(\alpha \vec{r})\right) \cdot\left(\hat{n}^{\prime} \times \vec{w}_{j}\left(\vec{r}^{\prime}\right)\right) d s d s^{\prime}\right\}
\end{aligned}
$$

This expression can be simplified by taking into account that $\alpha^{2}-1=(\alpha+1)(\alpha-1)$, obtaining:

$$
\begin{aligned}
I=\frac{1}{2} \lim _{\alpha \rightarrow 1}\left\{\frac{1}{\alpha-1}(\right. & \alpha^{2} \int_{S_{1}} \int_{V_{1}} \frac{R}{8 \pi} \nabla \times \vec{w}_{i}(\alpha \vec{r}) \cdot\left(\widehat{n}^{\prime} \times \vec{w}_{j}\left(\vec{r}^{\prime}\right)\right) d v d s^{\prime} \\
& +\alpha^{2} \int_{S_{1}} \int_{V_{1}} \frac{R}{8 \pi} \vec{w}_{i}(\alpha \vec{r}) \cdot\left(\widehat{n}^{\prime} \times \nabla^{\prime} \times \vec{w}_{j}\left(\vec{r}^{\prime}\right)\right) d v d s^{\prime} \\
& -\int_{S_{1}} \int_{V_{1}} \frac{R}{8 \pi}\left(\widehat{n} \times \vec{w}_{i}(\alpha \vec{r})\right) \cdot \nabla^{\prime} \times \vec{w}_{j}\left(\vec{r}^{\prime}\right) d v^{\prime} d s \\
& \left.\left.-\int_{S_{1}} \int_{V_{1}} \frac{R}{8 \pi}\left(\widehat{n} \times \nabla \times \vec{w}_{i}(\alpha \vec{r})\right) \cdot \vec{w}_{j}\left(\vec{r}^{\prime}\right) d v^{\prime} d s\right)\right\} \\
& -\int_{S_{1}} \int_{S_{1}} \frac{R}{8 \pi}\left(\widehat{n} \times \vec{w}_{i}(\vec{r})\right) \cdot\left(\widehat{n}^{\prime} \times \vec{w}_{j}\left(\vec{r}^{\prime}\right)\right) d s d s^{\prime}
\end{aligned}
$$

Now we consider the term inside the parenthesis in (4.123) as a continuous function of 
the scaling factor $\alpha$,

$$
\begin{aligned}
F(\alpha) \equiv & \alpha^{2} \int_{S_{1}} \int_{V_{1}} \frac{R}{8 \pi} \nabla \times \vec{w}_{i}(\alpha \vec{r}) \cdot\left(\widehat{n}^{\prime} \times \vec{w}_{j}\left(\vec{r}^{\prime}\right)\right) d v d s^{\prime} \\
& +\alpha^{2} \int_{S_{1}} \int_{V_{1}} \frac{R}{8 \pi} \vec{w}_{i}(\alpha \vec{r}) \cdot\left(\widehat{n}^{\prime} \times \nabla^{\prime} \times \vec{w}_{j}\left(\vec{r}^{\prime}\right)\right) d v d s^{\prime} \\
& -\int_{S_{1}} \int_{V_{1}} \frac{R}{8 \pi}\left(\widehat{n} \times \vec{w}_{i}(\alpha \vec{r})\right) \cdot \nabla^{\prime} \times \vec{w}_{j}\left(\vec{r}^{\prime}\right) d v^{\prime} d s \\
& -\int_{S_{1}} \int_{V_{1}} \frac{R}{8 \pi}\left(\widehat{n} \times \nabla \times \vec{w}_{i}(\alpha \vec{r})\right) \cdot \vec{w}_{j}\left(\vec{r}^{\prime}\right) d v^{\prime} d s
\end{aligned}
$$

Since, due to the indetermination of the kind $0 / 0$ we know that $F(1)=0$, then we can regard the limit in (4.123) as a partial derivative,

$$
\left.\frac{\partial F(\alpha)}{\partial \alpha}\right|_{\alpha=1}=\lim _{\alpha \rightarrow 1} \frac{F(\alpha)-F(1)}{\alpha-1},
$$

and thus, we have:

$$
\begin{aligned}
I=\frac{1}{2} \frac{\partial}{\partial \alpha}( & \alpha^{2} \int_{S_{1}} \int_{V_{1}} \frac{R}{8 \pi} \nabla \times \vec{w}_{i}(\alpha \vec{r}) \cdot\left(\widehat{n}^{\prime} \times \vec{w}_{j}\left(\vec{r}^{\prime}\right)\right) d v d s^{\prime} \\
& +\alpha^{2} \int_{S_{1}} \int_{V_{1}} \frac{R}{8 \pi} \vec{w}_{i}(\alpha \vec{r}) \cdot\left(\widehat{n}^{\prime} \times \nabla^{\prime} \times \vec{w}_{j}\left(\vec{r}^{\prime}\right)\right) d v d s^{\prime} \\
& -\int_{S_{1}} \int_{V_{1}} \frac{R}{8 \pi}\left(\widehat{n} \times \vec{w}_{i}(\alpha \vec{r})\right) \cdot \nabla^{\prime} \times \vec{w}_{j}\left(\vec{r}^{\prime}\right) d v^{\prime} d s \\
& \left.-\int_{S_{1}} \int_{V_{1}} \frac{R}{8 \pi}\left(\widehat{n} \times \nabla \times \vec{w}_{i}(\alpha \vec{r})\right) \cdot \vec{w}_{j}\left(\vec{r}^{\prime}\right) d v^{\prime} d s\right)_{\alpha=1} \\
& -\int_{S_{1}} \int_{S_{1}} \frac{R}{8 \pi}\left(\widehat{n} \times \vec{w}_{i}(\vec{r})\right) \cdot\left(\hat{n}^{\prime} \times \vec{w}_{j}\left(\vec{r}^{\prime}\right)\right) d s d s^{\prime}
\end{aligned}
$$

Next, introducing the new symbol $\underline{\vec{w}}_{i}(\vec{r})=\left.\frac{\partial}{\partial \alpha} \vec{w}_{i}(\alpha \vec{r})\right|_{\alpha=1}$, and differentiating we obtain:

$$
\begin{aligned}
I & =\int_{S_{1}} \int_{V_{1}} \frac{R}{8 \pi} \nabla \times \vec{w}_{i}(\vec{r}) \cdot\left(\widehat{n}^{\prime} \times \vec{w}_{j}\left(\vec{r}^{\prime}\right)\right) d v d s^{\prime}+\int_{S_{1}} \int_{V_{1}} \frac{R}{8 \pi} \vec{w}_{i}(\vec{r}) \cdot\left(\widehat{n}^{\prime} \times \nabla^{\prime} \times \vec{w}_{j}\left(\vec{r}^{\prime}\right)\right) d v d s^{\prime} \\
& +\frac{1}{2} \int_{S_{1}} \int_{V_{1}} \frac{R}{8 \pi} \nabla \times \underline{w}_{i}(\vec{r}) \cdot\left(\widehat{n}^{\prime} \times \vec{w}_{j}\left(\vec{r}^{\prime}\right)\right) d v d s^{\prime}+\frac{1}{2} \int_{S_{1}} \int_{V_{1}} \frac{R}{8 \pi} \vec{w}_{i}(\vec{r}) \cdot\left(\widehat{n}^{\prime} \times \nabla^{\prime} \times \vec{w}_{j}\left(\vec{r}^{\prime}\right)\right) d v d s^{\prime} \\
& -\frac{1}{2} \int_{S_{1}} \int_{V_{1}} \frac{R}{8 \pi}\left(\widehat{n} \times \nabla \times \underline{\vec{w}}_{i}(\vec{r})\right) \cdot \vec{w}_{j}\left(\vec{r}^{\prime}\right) d v^{\prime} d s-\frac{1}{2} \int_{S_{1}} \int_{S_{1}} \frac{R}{8 \pi}\left(\widehat{n} \times \vec{w}_{i}(\vec{r})\right) \cdot\left(\widehat{n}^{\prime} \times \vec{w}_{j}\left(\vec{r}^{\prime}\right)\right) d s d s^{\prime} \\
& -\int_{S_{1}} \int_{S_{1}} \frac{R}{8 \pi}\left(\widehat{n} \times \vec{w}_{i}(\vec{r})\right) \cdot\left(\widehat{n}^{\prime} \times \vec{w}_{j}\left(\vec{r}^{\prime}\right)\right) d s d s^{\prime}
\end{aligned}
$$


We can observe that expression (4.127) may be a good solution for computing $L_{i j}^{\text {sing }}$ when $k_{i}=k_{j}$. Nevertheless, since $\mathbf{L}^{\text {sing }}$ is a symmetric matrix, we should obtain a symmetric expression with repspect to the indexes $i$ and $j$. We know that when $k_{i}=k_{j}$ the expression (4.117) becomes an indeterminate form of the kind $0 / 0$, so this implies that the following term vanishes:

$$
\begin{array}{r}
k_{i}^{2}\left[\int_{S_{1}} \int_{V_{1}} \frac{R}{8 \pi} \nabla \times \vec{w}_{i}(\vec{r}) \cdot\left(\widehat{n}^{\prime} \times \vec{w}_{j}\left(\vec{r}^{\prime}\right)\right) d v d s^{\prime}+\int_{S_{1}} \int_{V_{1}} \frac{R}{8 \pi} \vec{w}_{i}(\vec{r}) \cdot\left(\widehat{n}^{\prime} \times \nabla^{\prime} \times \vec{w}_{j}\left(\vec{r}^{\prime}\right)\right) d v d s^{\prime}\right] \\
-k_{j}^{2}\left[\int_{S_{1}} \int_{V_{1}} \frac{R}{8 \pi}\left(\widehat{n} \times \vec{w}_{i}(\vec{r})\right) \cdot \nabla^{\prime} \times \vec{w}_{j}\left(\vec{r}^{\prime}\right) d v^{\prime} d s-\int_{S_{1}} \int_{V_{1}} \frac{R}{8 \pi}\left(\widehat{n} \times \nabla \times \vec{w}_{i}(\vec{r})\right) \cdot \vec{w}_{j}\left(\vec{r}^{\prime}\right) d v^{\prime} d s\right]
\end{array}
$$

and thus,

$$
\begin{aligned}
& \int_{S_{1}} \int_{V_{1}} \frac{R}{8 \pi} \nabla \times \vec{w}_{i}(\vec{r}) \cdot\left(\widehat{n}^{\prime} \times \vec{w}_{j}\left(\vec{r}^{\prime}\right)\right) d v d s^{\prime}+\int_{S_{1}} \int_{V_{1}} \frac{R}{8 \pi} \vec{w}_{i}(\vec{r}) \cdot\left(\widehat{n}^{\prime} \times \nabla^{\prime} \times \vec{w}_{j}\left(\vec{r}^{\prime}\right)\right) d v d s^{\prime}= \\
& \int_{S_{1}} \int_{V_{1}} \frac{R}{8 \pi}\left(\widehat{n} \times \vec{w}_{i}(\vec{r})\right) \cdot \nabla^{\prime} \times \vec{w}_{j}\left(\vec{r}^{\prime}\right) d v^{\prime} d s+\int_{S_{1}} \int_{V_{1}} \frac{R}{8 \pi}\left(\widehat{n} \times \nabla \times \vec{w}_{i}(\vec{r})\right) \cdot \vec{w}_{j}\left(\vec{r}^{\prime}\right) d v^{\prime} d s
\end{aligned}
$$

Therefore, we can substitute the first two integrals in (4.127) by

$$
\begin{aligned}
& \int_{S_{1}} \int_{V_{1}} \frac{R}{8 \pi} \nabla \times \vec{w}_{i}(\vec{r}) \cdot\left(\widehat{n}^{\prime} \times \vec{w}_{j}\left(\vec{r}^{\prime}\right)\right) d v d s^{\prime}+\int_{S_{1}} \int_{V_{1}} \frac{R}{8 \pi} \vec{w}_{i}(\vec{r}) \cdot\left(\widehat{n}^{\prime} \times \nabla^{\prime} \times \vec{w}_{j}\left(\vec{r}^{\prime}\right)\right) d v d s^{\prime}= \\
& \frac{1}{2}\left[\int_{S_{1}} \int_{V_{1}} \frac{R}{8 \pi} \nabla \times \vec{w}_{i}(\vec{r}) \cdot\left(\widehat{n}^{\prime} \times \vec{w}_{j}\left(\vec{r}^{\prime}\right)\right) d v d s^{\prime}+\int_{S_{1}} \int_{V_{1}} \frac{R}{8 \pi} \vec{w}_{i}(\vec{r}) \cdot\left(\widehat{n}^{\prime} \times \nabla^{\prime} \times \vec{w}_{j}\left(\vec{r}^{\prime}\right)\right) d v d s^{\prime}\right. \\
& \left.+\int_{S_{1}} \int_{V_{1}} \frac{R}{8 \pi}\left(\widehat{n} \times \vec{w}_{i}(\vec{r})\right) \cdot \nabla^{\prime} \times \vec{w}_{j}\left(\vec{r}^{\prime}\right) d v^{\prime} d s+\int_{S_{1}} \int_{V_{1}} \frac{R}{8 \pi}\left(\widehat{n} \times \nabla \times \vec{w}_{i}(\vec{r})\right) \cdot \vec{w}_{j}\left(\vec{r}^{\prime}\right) d v^{\prime} d s\right],
\end{aligned}
$$

and finally obtain:

$$
\begin{aligned}
L_{i j}^{\text {sing }} & =\int_{S_{1}} \int_{V_{1}} \frac{R}{8 \pi} \nabla \times \frac{\overrightarrow{\underline{w}}_{i}(\vec{r})+\vec{w}_{i}(\vec{r})}{2} \cdot\left(\widehat{n}^{\prime} \times \vec{w}_{j}\left(\vec{r}^{\prime}\right)\right) d v d s^{\prime} \\
& +\int_{S_{1}} \int_{V_{1}} \frac{R}{8 \pi} \frac{\left(\underline{\vec{w}}_{i}(\vec{r})+\vec{w}_{i}(\vec{r})\right)}{2} \cdot\left(\widehat{n}^{\prime} \times \nabla^{\prime} \times \vec{w}_{j}\left(\vec{r}^{\prime}\right)\right) d v d s^{\prime} \\
& -\int_{S_{1}} \int_{V_{1}} \frac{R}{8 \pi}\left(\widehat{n} \times \frac{\underline{\vec{w}}_{i}(\vec{r})-\vec{w}_{i}(\vec{r})}{2}\right) \cdot \nabla^{\prime} \times \vec{w}_{j}\left(\vec{r}^{\prime}\right) d v^{\prime} d s \\
& -\int_{S_{1}} \int_{V_{1}} \frac{R}{8 \pi}\left(\widehat{n} \times \nabla \times \frac{\vec{w}_{i}(\vec{r})-\vec{w}_{i}(\vec{r})}{2}\right) \cdot \vec{w}_{j}\left(\vec{r}^{\prime}\right) d v^{\prime} d s \\
& -\int_{S_{1}} \int_{S_{1}} \frac{R}{8 \pi}\left(\widehat{n} \times \vec{w}_{i}(\vec{r})\right) \cdot\left(\widehat{n}^{\prime} \times \vec{w}_{j}\left(\vec{r}^{\prime}\right)\right) d s d s^{\prime}
\end{aligned}
$$


In this case, due to the angular dependence of the integrands, we can also use Appendix B to reduce one dimension the integrals in this last expression. Therefore, the integrals involved in the calculation of $\mathbf{L}^{\text {sing }}$ have been finally reduced to four-dimension regular integrals.

\section{B. Computation of the $\mathrm{L}^{\text {reg }}$ matrix}

The expression for the $\mathbf{L}^{\text {reg }}$ matrix entries is:

$$
L_{i j}^{r e g} \equiv \int_{V_{1}} \int_{V_{1}} \vec{w}_{i}(\vec{r}) \cdot \underline{G}_{0, r}^{A}\left(\vec{r}, \vec{r}^{\prime}\right) \cdot \vec{w}_{j}\left(\vec{r}^{\prime}\right) d v d v^{\prime}
$$

A similar procedure to the one just described for the $\mathbf{L}^{\text {sing }}$ terms has been followed to transform the previous integral. Using the Green's Second Theorem, a first transformation of one of the volume integrals can be performed:

$$
\begin{aligned}
& \int_{V_{1}} \vec{w}_{i}(\vec{r}) \cdot \underline{G}_{o, r}^{A}\left(\vec{r}, \vec{r}^{\prime}\right) d v=k_{i}^{-2}\left\{\int_{V_{1}} \vec{w}_{i}(\vec{r}) \cdot \nabla \times \nabla \times \underline{G}_{o, r}^{A}\left(\vec{r}, \vec{r}^{\prime}\right) d v\right. \\
& \left.+\int_{S_{1}}\left(\widehat{n} \times \vec{w}_{i}(\vec{r})\right) \cdot \nabla \times \underline{G}_{o, r}^{A}\left(\vec{r}, \vec{r}^{\prime}\right) d s+\int_{S_{1}}\left(\widehat{n} \times \nabla \times \vec{w}_{i}(\vec{r})\right) \cdot \underline{G}_{o, r}^{A}\left(\vec{r}, \vec{r}^{\prime}\right) d s\right\}
\end{aligned}
$$

Introducing in this expression the identity satisfied by the dyadic $\underline{G}_{o, r}^{A}\left(\vec{r}, \vec{r}^{\prime}\right)$ (see section E.2 of Appendix E):

$$
\nabla \times \nabla \times \underline{G}_{o, r}^{A}\left(\vec{r}, \vec{r}^{\prime}\right)=-\nabla \nabla^{\prime} g_{r}^{e}\left(\vec{r}, \vec{r}^{\prime}\right)
$$

we can rewrite the integral,

$$
\begin{aligned}
& \int_{V_{1}} \vec{w}_{i}(\vec{r}) \cdot \underline{G}_{o, r}^{A}\left(\vec{r}, \vec{r}^{\prime}\right) d v=k_{i}^{-2}\left\{-\nabla^{\prime} \int_{S_{1}}\left(\vec{w}_{i}(\vec{r}) \cdot \widehat{n}\right) g_{r}^{e}\left(\vec{r}, \vec{r}^{\prime}\right) d s\right. \\
& \left.+\int_{S_{1}}\left(\widehat{n} \times \vec{w}_{i}(\vec{r})\right) \cdot \nabla \times \underline{G}_{o, r}^{A}\left(\vec{r}, \vec{r}^{\prime}\right) d s+\int_{S_{1}}\left(\widehat{n} \times \nabla \times \vec{w}_{i}(\vec{r})\right) \cdot \underline{G}_{o, r}^{A}\left(\vec{r}, \vec{r}^{\prime}\right) d s\right\}
\end{aligned}
$$

To obtain the first surface integral in the right hand sside of this expression, we have taken into account that $\left\{\vec{w}_{i}\right\}$ are solenoidal functions, and thus, by means of the Gauss theorem, we have:

$$
\begin{aligned}
& \int_{V_{1}} \vec{w}_{i}(\vec{r}) \cdot \nabla \nabla^{\prime} g_{r}^{e}\left(\vec{r}, \vec{r}^{\prime}\right) d v=\int_{V_{1}} \nabla^{\prime} \nabla g_{r}^{e}\left(\vec{r}, \vec{r}^{\prime}\right) \cdot \vec{w}_{i}(\vec{r}) d v \\
& =\nabla^{\prime} \int_{V_{1}} \nabla g_{r}^{e}\left(\vec{r}, \vec{r}^{\prime}\right) \cdot \vec{w}_{i}(\vec{r}) d v=\nabla^{\prime} \int_{S_{1}} g_{r}^{e}\left(\vec{r}, \vec{r}^{\prime}\right)\left(\vec{w}_{i}(\vec{r}) \cdot \widehat{n}\right) d s
\end{aligned}
$$


Now we introduce (4.131) into (4.99b) to rewrite the entries of the $\mathbf{L}^{\text {reg }}$ matrix as follows:

$$
\begin{aligned}
L_{i j}^{r e g}= & k_{i}^{-2}\left\{-\int_{V_{1}} \int_{S_{1}} \nabla^{\prime}\left[\left(\vec{w}_{i}(\vec{r}) \cdot \widehat{n}\right) g_{r}^{e}\left(\vec{r}, \vec{r}^{\prime}\right)\right] \cdot \vec{w}_{j}\left(\vec{r}^{\prime}\right) d s d v^{\prime}\right. \\
& +\int_{V_{1}} \int_{S_{1}}\left(\widehat{n} \times \vec{w}_{i}(\vec{r})\right) \cdot \nabla \times \underline{G}_{o, r}^{A}\left(\vec{r}, \vec{r}^{\prime}\right) \cdot \vec{w}_{j}\left(\vec{r}^{\prime}\right) d s d v^{\prime} \\
& \left.+\int_{V_{1}} \int_{S_{1}}\left(\widehat{n} \times \nabla \times \vec{w}_{i}(\vec{r})\right) \cdot \underline{G}_{o, r}^{A}\left(\vec{r}, \vec{r}^{\prime}\right) \cdot \vec{w}_{j}\left(\vec{r}^{\prime}\right) d s d v^{\prime}\right\} \\
\equiv & k_{i}^{-2}\left\{-I_{i j}^{(1)}+I_{i j}^{(2)}+I_{i j}^{(3)}\right\}
\end{aligned}
$$

Analogously to the procedure followed to obtain the equation (4.132), we can transform the integral $I_{i j}^{(1)}$ using the Gauss theorem:

$$
I_{i j}^{(1)}=\int_{S_{1}} \int_{S_{1}}\left(\vec{w}_{i}(\vec{r}) \cdot \widehat{n}\right) \cdot g_{r}^{e}\left(\vec{r}, \vec{r}^{\prime}\right) \cdot\left(\vec{w}_{j}\left(\vec{r}^{\prime}\right) \cdot \widehat{n}^{\prime}\right) d s d s^{\prime}
$$

In order to reformulate the integrals $I_{i j}^{(2)}$ and $I_{i j}^{(3)}$, let us first consider the following expression:

$$
\begin{aligned}
& \int_{V_{1}} G_{o, r}^{A}\left(\vec{r}, \vec{r}^{\prime}\right) \cdot \vec{w}_{j}\left(\vec{r}^{\prime}\right) d v^{\prime}=k_{j}^{-2}\left\{\int_{V_{1}} \nabla^{\prime} \times \nabla^{\prime} \times \underline{G}_{o, r}^{A}\left(\vec{r}, \vec{r}^{\prime}\right) \cdot \vec{w}_{j}\left(\vec{r}^{\prime}\right) d v^{\prime}\right. \\
& \left.\quad+\int_{S_{1}} \nabla^{\prime} \times \underline{G}_{o, r}^{A}\left(\vec{r}, \vec{r}^{\prime}\right) \cdot\left(\widehat{n}^{\prime} \times \vec{w}_{j}\left(\vec{r}^{\prime}\right)\right) d s^{\prime}+\int_{S_{1}}{ }_{o, r}^{A}\left(\vec{r}, \vec{r}^{\prime}\right) \cdot\left(\widehat{n}^{\prime} \times \nabla^{\prime} \times \vec{w}_{j}\left(\vec{r}^{\prime}\right)\right) d s^{\prime}\right\}
\end{aligned}
$$

where we have used equation (4.1) and the second vector-dyadic Green's theorem (see (C.20)). Now, considering the identities satisfied by the dyadic $\underline{G}_{o, r}^{A}\left(\vec{r}, \vec{r}^{\prime}\right)$ (see section E.2 of Appendix E):

$$
\begin{aligned}
\nabla^{\prime} \times \nabla^{\prime} \times \underline{G}_{o, r}^{A}\left(\vec{r}, \vec{r}^{\prime}\right) & =-\nabla \nabla^{\prime} g_{r}^{e}\left(\vec{r}, \vec{r}^{\prime}\right) \\
\nabla^{\prime} \times \underline{G}_{o, r}^{A}\left(\vec{r}, \vec{r}^{\prime}\right) & =\nabla \times \underline{G}_{o, r}^{F}\left(\vec{r}, \vec{r}^{\prime}\right)
\end{aligned}
$$

We can rewrite the expression (4.135) as:

$$
\begin{aligned}
& \int_{V_{1}}^{G_{o, r}^{A}}\left(\vec{r}, \vec{r}^{\prime}\right) \cdot \vec{w}_{j}\left(\vec{r}^{\prime}\right) d v^{\prime}=k_{j}^{-2}\left\{-\nabla \int_{S_{1}} g_{r}^{e}\left(\vec{r}, \vec{r}^{\prime}\right)\left(\hat{n}^{\prime} \cdot \vec{w}_{j}\left(\vec{r}^{\prime}\right)\right) d s^{\prime}\right. \\
& \left.\quad+\int_{S_{1}} \nabla \times \underline{G}_{o, r}^{F}\left(\vec{r}, \vec{r}^{\prime}\right) \cdot\left(\hat{n}^{\prime} \times \vec{w}_{j}\left(\vec{r}^{\prime}\right)\right) d s^{\prime}+\int_{S_{1}} \underline{G}_{o, r}^{A}\left(\vec{r}, \vec{r}^{\prime}\right) \cdot\left(\widehat{n}^{\prime} \times \nabla^{\prime} \times \vec{w}_{j}\left(\vec{r}^{\prime}\right)\right) d s^{\prime}\right\}
\end{aligned}
$$

This way, by introducing equation (4.138) into the expressions of $I_{i j}^{(2)}$ and $I_{i j}^{(3)}$, we have:

$$
\begin{aligned}
I_{i j}^{(2)}=k_{j}^{-2}\{ & -\int_{S_{1}} \int_{S_{1}}\left(\widehat{n} \times \vec{w}_{i}(\vec{r})\right) \cdot \nabla \nabla^{\prime} g_{r}^{m}\left(\vec{r}, \vec{r}^{\prime}\right) \cdot\left(\widehat{n}^{\prime} \times \vec{w}_{j}\left(\vec{r}^{\prime}\right)\right) d s d s^{\prime} \\
& \left.+\int_{S_{1}} \int_{S_{1}}\left(\widehat{n} \times \vec{w}_{i}(\vec{r})\right) \cdot \nabla \times \underline{G}_{o, r}^{A}\left(\vec{r}, \vec{r}^{\prime}\right) \cdot\left(\widehat{n}^{\prime} \times \nabla^{\prime} \times \vec{w}_{j}\left(\vec{r}^{\prime}\right)\right) d s d s^{\prime}\right\},
\end{aligned}
$$


and

$$
\begin{aligned}
I_{i j}^{(3)}= & k_{j}^{-2}\left\{-\int_{S_{1}} \int_{S_{1}}\left(\widehat{n} \times \nabla \times \vec{w}_{i}(\vec{r})\right) \cdot \nabla g_{r}^{e}\left(\vec{r}, \vec{r}^{\prime}\right) \cdot\left(\widehat{n}^{\prime} \cdot \vec{w}_{j}\left(\vec{r}^{\prime}\right)\right) d s d s^{\prime}\right. \\
& +\int_{S_{1}} \int_{S_{1}}\left(\widehat{n} \times \nabla \times \vec{w}_{i}(\vec{r})\right) \cdot \nabla \times \underline{G}_{o, r}^{F}\left(\vec{r}, \vec{r}^{\prime}\right) \cdot\left(\widehat{n}^{\prime} \times \vec{w}_{j}\left(\vec{r}^{\prime}\right)\right) d s d s^{\prime} \\
& \left.+\int_{S_{1}} \int_{S_{1}}\left(\widehat{n} \times \nabla \times \vec{w}_{i}(\vec{r})\right) \cdot \underline{G}_{o, r}^{A}\left(\vec{r}, \vec{r}^{\prime}\right) \cdot\left(\hat{n}^{\prime} \times \nabla^{\prime} \times \vec{w}_{j}\left(\vec{r}^{\prime}\right)\right) d s d s^{\prime}\right\}
\end{aligned}
$$

where we have used to obtain $I_{i j}^{(2)}$, the identity $\nabla \times \nabla \times \underline{G}_{o, r}^{F}\left(\vec{r}, \vec{r}^{\prime}\right)=-\nabla \nabla^{\prime} g_{r}^{m}\left(\vec{r}, \vec{r}^{\prime}\right)$ (see section E.2 of Appendix E).

The terms containing $\nabla \nabla^{\prime} g_{r}^{m}\left(\vec{r}, \vec{r}^{\prime}\right)$ and $\nabla g_{r}^{e}\left(\vec{r}, \vec{r}^{\prime}\right)$ need further development. For instance, in order to arrange the term containing $\nabla \nabla^{\prime} g_{r}^{m}$ in the expression of $I_{i j}^{(2)}$, it must be noticed that $\left(\widehat{n} \times \vec{w}_{i}(\vec{r})\right)$ is a vector tangent to the surface $S_{1}$. In consequence, it can be written:

$$
\begin{aligned}
& \int_{S_{1}} \int_{S_{1}}\left(\widehat{n} \times \vec{w}_{i}(\vec{r})\right) \cdot \nabla \nabla^{\prime} g_{r}^{m}\left(\vec{r}, \vec{r}^{\prime}\right) \cdot\left(\widehat{n}^{\prime} \times \vec{w}_{j}\left(\vec{r}^{\prime}\right)\right) d s d s^{\prime}= \\
& \int_{S_{1}} \int_{S_{1}}\left(\widehat{n} \times \vec{w}_{i}(\vec{r})\right) \cdot \nabla_{s} \nabla_{s}^{\prime} g_{r}^{m}\left(\vec{r}, \vec{r}^{\prime}\right) \cdot\left(\widehat{n}^{\prime} \times \vec{w}_{j}\left(\vec{r}^{\prime}\right)\right) d s d s^{\prime}
\end{aligned}
$$

Then, applying the Gauss theorem on a surface, it is obtained:

$$
\begin{aligned}
& \int_{S_{1}} \int_{S_{1}}\left(\widehat{n} \times \vec{w}_{i}(\vec{r})\right) \cdot \nabla \nabla^{\prime} g_{r}^{m}\left(\vec{r}, \vec{r}^{\prime}\right) \cdot\left(\widehat{n}^{\prime} \times \vec{w}_{j}\left(\vec{r}^{\prime}\right)\right) d s d s^{\prime}= \\
& \int_{S_{1}} \int_{S_{1}}\left(\nabla_{s} \cdot\left(\widehat{n} \times \vec{w}_{i}(\vec{r})\right)\right) \cdot g_{r}^{m}\left(\vec{r}, \vec{r}^{\prime}\right) \cdot\left(\nabla_{s}^{\prime} \cdot\left(\widehat{n}^{\prime} \times \vec{w}_{j}\left(\vec{r}^{\prime}\right)\right)\right) d s d s^{\prime}
\end{aligned}
$$

In order to reduce the integral containing $\nabla g_{r e g}^{e}$ in the expression of $I_{i j}^{(3)}$, let us apply the the Vector Green's First Theorem (eq. (C.7)) to the following expression:

$$
\begin{aligned}
& \int_{V_{1}} \vec{w}_{i}(\vec{r}) \cdot \nabla g_{r}^{e}\left(\vec{r}, \vec{r}^{\prime}\right) d v=k_{i}^{-2} \int_{V_{1}}\left(\nabla \times \nabla \times \vec{w}_{i}(\vec{r})\right) \cdot \nabla g_{r}^{e}\left(\vec{r}, \vec{r}^{\prime}\right) d v \\
& =k_{i}^{-2}\left[\int_{V_{1}}\left(\nabla \times \vec{w}_{i}(\vec{r})\right) \cdot\left(\nabla \times \nabla g_{r}^{e}\left(\vec{r}, \vec{r}^{\prime}\right)\right) d v-\int_{S_{1}} \widehat{n} \cdot\left(\nabla g_{r}^{e}\left(\vec{r}, \vec{r}^{\prime}\right) \times \nabla \times \vec{w}_{i}(\vec{r})\right) d s\right] \\
& =k_{i}^{-2} \int_{S_{1}}\left(\widehat{n} \times \nabla \times \vec{w}_{i}(\vec{r})\right) \cdot \nabla g_{r}^{e}\left(\vec{r}, \vec{r}^{\prime}\right) d s
\end{aligned}
$$

We can write:

$$
\begin{aligned}
& \int_{S_{1}} \int_{S_{1}}\left(\widehat{n} \times \nabla \times \vec{w}_{i}(\vec{r})\right) \cdot \nabla g_{r}^{e}\left(\vec{r}, \vec{r}^{\prime}\right) \cdot\left(\widehat{n}^{\prime} \cdot \vec{w}_{j}\left(\vec{r}^{\prime}\right)\right) d s d s^{\prime}= \\
& k_{i}^{2} \int_{V_{1}} \int_{S_{1}} \vec{w}_{i}(\vec{r}) \cdot \nabla g_{r}^{e}\left(\vec{r}, \vec{r}^{\prime}\right) \cdot\left(\widehat{n}^{\prime} \cdot \vec{w}_{j}\left(\vec{r}^{\prime}\right)\right) d v d s^{\prime}
\end{aligned}
$$


Therefore, applying now the Gauss theorem to the volume integral we finally get

$$
\begin{aligned}
& \int_{S_{1}} \int_{S_{1}}\left(\widehat{n} \times \nabla \times \vec{w}_{i}(\vec{r})\right) \cdot \nabla g_{r}^{e}\left(\vec{r}, \vec{r}^{\prime}\right) \cdot\left(\widehat{n}^{\prime} \cdot \vec{w}_{j}\left(\vec{r}^{\prime}\right)\right) d s d s^{\prime}= \\
& k_{i}^{2} \int_{S_{1}} \int_{S_{1}}\left(\widehat{n} \cdot \vec{w}_{i}(\vec{r})\right) \cdot g_{r}^{e}\left(\vec{r}, \vec{r}^{\prime}\right) \cdot\left(\widehat{n}^{\prime} \cdot \vec{w}_{i}(\vec{r})\right) d s d s^{\prime}
\end{aligned}
$$

Finally, introducing the equations (4.141) and (4.143) into (4.139) and (4.140) respectively, and substituting the reduced expressions for $I_{i j}^{(1)}, I_{i j}^{(2)}$ and $I_{i j}^{(3)}$ into $L_{i j}^{r e g}$, we obtain the following expression in terms of double surface integrals:

$$
\begin{aligned}
L_{i j}^{r e g}=\frac{1}{k_{i}^{2} k_{j}^{2}}\{ & -\int_{S_{1}} \int_{S_{1}} \nabla_{s} \cdot\left(\widehat{n} \times \vec{w}_{i}(\vec{r})\right) \cdot g_{r}^{m}\left(\vec{r}, \vec{r}^{\prime}\right) \cdot \nabla_{s}^{\prime} \cdot\left(\widehat{n}^{\prime} \times \vec{w}_{j}\left(\vec{r}^{\prime}\right)\right) d s d s^{\prime} \\
& +\int_{S_{1}} \int_{S_{1}}\left(\widehat{n} \times \nabla \times \vec{w}_{i}(\vec{r})\right) \cdot \underline{G}_{o, r}^{A}\left(\vec{r}, \vec{r}^{\prime}\right) \cdot\left(\widehat{n}^{\prime} \times \nabla^{\prime} \times \vec{w}_{j}\left(\vec{r}^{\prime}\right)\right) d s d s^{\prime} \\
& +\int_{S_{1}} \int_{S_{1}}\left(\widehat{n} \times \vec{w}_{i}(\vec{r})\right) \cdot \nabla \times \underline{G}_{o, r}^{A}\left(\vec{r}, \vec{r}^{\prime}\right) \cdot\left(\widehat{n}^{\prime} \times \nabla^{\prime} \times \vec{w}_{j}\left(\vec{r}^{\prime}\right)\right) d s d s^{\prime} \\
& \left.+\int_{S_{1}} \int_{S_{1}}\left(\widehat{n} \times \vec{w}_{j}(\vec{r})\right) \cdot \nabla \times \underline{G}_{o, r}^{A}\left(\vec{r}, \vec{r}^{\prime}\right) \cdot\left(\widehat{n}^{\prime} \times \nabla^{\prime} \times \vec{w}_{i}(\vec{r})\right) d s d s^{\prime}\right\} \\
& -\frac{k_{i}^{2}+k_{j}^{2}}{k_{i}^{2} k_{j}^{2}} \int_{S_{1}} \int_{S_{1}}\left(\widehat{n} \cdot \vec{w}_{i}(\vec{r})\right) \cdot g_{r}^{e}\left(\vec{r}, \vec{r}^{\prime}\right) \cdot\left(\widehat{n}^{\prime} \cdot \vec{w}_{j}\left(\vec{r}^{\prime}\right)\right) d s d s^{\prime}
\end{aligned}
$$

Therefore the $\mathbf{L}^{\text {reg }}$ matrix has been expressed in terms of 4-D surface integrals, avoiding in such a a way the necessity of performing 3-D meshes in the dielectric body.

\subsubsection{Computation of the $G, T, W$ and $F$ matrices}

So far in this section we have presented the computation of the $\mathbf{D}, \mathbf{S}, \mathbf{C}$ and $\mathbf{L}$ matrices. According to the system of equations described in (3.37), it can be easily observed that these are the matrices involved in the solution of the homogeneous eigenvalue problem (3.42). As it has been explained in section (3.4), the solution of this eigenvalue problem implies that the impressed voltage is zero, this is, the resonant structure with its access ports closed. Therefore, at this point we are able to calculate the resonant frequencies of the rectangular cavity loaded with the cylindrical dielectric resonator and its modal field distribution. However, if we want to calculate the GAM of the structure under analysis, we still have to consider the aperture of the access ports. For this purpose, we have to proceed with the computation of the $\mathbf{G}, \mathbf{T}, \mathbf{F}$ and $\mathbf{W}$ matrices collected in Table 3.1. We recall that this matrices are directly involved in the computation of the GAM (3.50) through the calculus of the matrices $\mathrm{Y}^{\mathrm{A}}$ and $\mathrm{Y}^{\mathrm{B}}$ (see (3.24)).

In this work we have considered the aperture of the four lateral faces and the top cap of the rectangular cavity under study (see Fig. 4.7). Note that following the notation introduced 


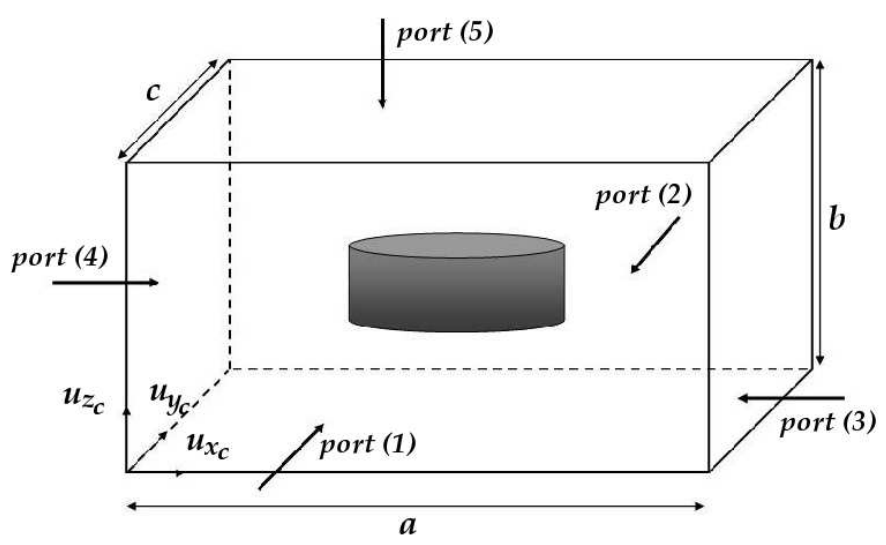

Figure 4.7: Rectangular cavity loaded with a cylindrical DR opened by its five access ports.

in the expression (3.51), we consider $N_{\xi}$ modes at each access port $(\xi)$, being $N$ the total number of modes at the ports (see table 3.1):

$$
N=\sum_{\xi}^{5} N_{\xi}
$$

Therefore, using this notation, the calculation of the $\mathbf{G}, \mathbf{T}, \mathbf{W}$ and $\mathbf{F}$ matrices can be performed by blocks. These blocks represent the interaction of each access port, and we can express their entries as:

$$
\begin{aligned}
G_{m n}^{(\gamma, \xi)} & =\int_{S_{\gamma}} \int_{S_{\xi}} \nabla_{s} \cdot \vec{h}_{m}^{(\gamma)}(\vec{r}) g^{m}\left(\vec{r}, \vec{r}^{\prime}\right) \nabla_{s}^{\prime} \cdot \vec{h}_{n}^{(\xi)}\left(\vec{r}^{\prime}\right) d s d s^{\prime} \\
T_{m n}^{(\gamma, \xi)} & =\int_{S_{\gamma}} \int_{S_{\xi}} \vec{h}_{m}^{(\gamma)}(\vec{r}) \cdot \underline{G}_{o}^{F}\left(\vec{r}, \vec{r}^{\prime}\right) \cdot \vec{h}_{n}^{(\xi)}\left(\vec{r}^{\prime}\right) d s d s^{\prime} \\
W_{m n}^{(\xi)} & =\int_{V_{1}} \int_{S_{\xi}} \vec{w}_{m}(\vec{r}) \cdot \nabla \times \underline{G}_{o}^{F}\left(\vec{r}, \vec{r}^{\prime}\right) \cdot \vec{h}_{n}^{(\xi)}\left(\vec{r}^{\prime}\right) d s^{\prime} d v \\
F_{m n}^{(\xi)} & =\int_{S_{\xi}} \vec{H}_{m}(\vec{r}) \cdot \vec{h}_{n}^{(\xi)}(\vec{r}) d s
\end{aligned}
$$

In theses expressions $\gamma, \xi=1, \ldots, 5$ represent the access ports (see Fig. 4.7); $S_{\xi}$ is the rectangular surface associated to the cross section of the port $(\xi) ; n=1, \ldots, N_{\xi} ; m=$ $1, \ldots, N_{\gamma}$ for the $\mathbf{G}$ and $\mathbf{T}$ matrices; $m=1, \ldots, Q$ for the $\mathbf{W}$ matrix, where $Q$ is the number of basis functions used; $m=1, \ldots, M$ for the $\mathbf{F}$ matrix, where $M$ is the number of the rectangular cavity modes used in the expansion of the dyadic Green's function; $\vec{h}_{n}^{(\xi)}(\vec{r})$ is the $n$-th normalized magnetic modal vector associated to the port $(\xi) ; g^{m}\left(\vec{r}, \vec{r}^{\prime}\right)$ is the static Green's function for the magnetic scalar potential of the rectangular cavity; $\underline{G}_{o}^{F}\left(\vec{r}, \vec{r}^{\prime}\right)$ is the quasi-static dyadic Green's function for the electric vector potential of the rectangular 
cavity; $\vec{H}_{m}(\vec{r})$ is the $m$-th magnetic resonant mode of the empty rectangular cavity; $\vec{w}_{m}(\vec{r})$ is the $m$-th basis function used to expand the polarization vector by the MoM.

Let us consider now the geometry of the structure under analysis in Fig. 4.8. It can be observed that, in the coordinate system $\left(x_{1}, y_{1}, z_{1}\right)$, we have considered that the access port (1) of the structure is located at $z_{1}=0$; the port (2) is on the plane $z_{1}=c$; the port (3) on $x_{1}=0$; the port (4) on $x_{1}=a$, and the port (5) on the plane defined by $y_{1}=b$.

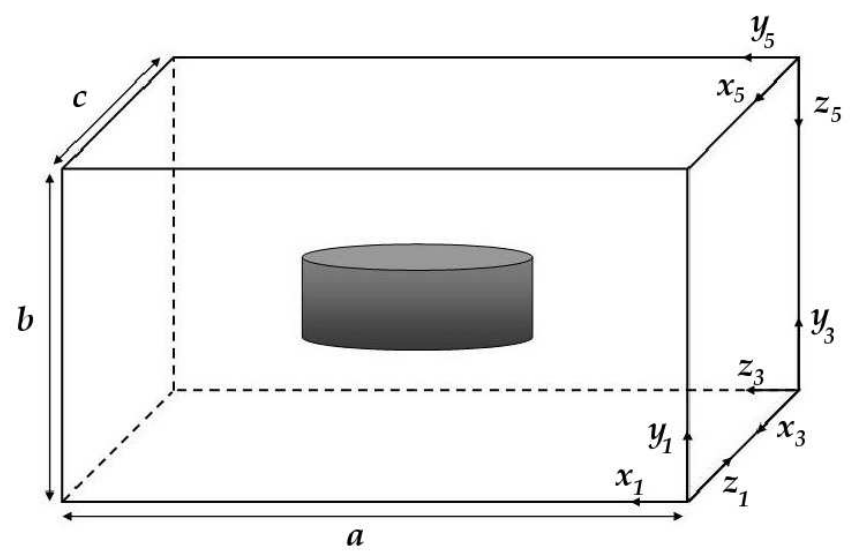

Figure 4.8: Rectangular cavity loaded with a cylindrical DR opened by its five access ports.

To compute the entries of the matrices (4.146)-(4.149), it is necessary to know the expressions of the magnetic modal vectors $\vec{h}_{n}^{(\xi)}(\vec{r})$ at each access port of the rectangular cavity. For this purpose, let us consider $\left(x_{\xi}, y_{\xi}\right)$ the transverse coordinates defined at the port $(\xi)$, being $z_{\xi}$ the axial coordinate. Thus, we can write [86]:

$$
\vec{h}_{n}^{(\xi)}(\vec{r})=\mathcal{N}_{x_{\xi}, n} F_{x_{\xi}, n}\left(x_{\xi}, y_{\xi}\right) \widehat{x}_{\xi}+\mathcal{N}_{y_{\xi}, n} F_{y_{\xi}, n}\left(x_{\xi}, y_{\xi}\right) \widehat{y}_{\xi}
$$

where the normalization factors $\mathcal{N}_{x_{\xi}, n}$ and $\mathcal{N}_{y_{\xi}, n}$, as well as the scalar functions $F_{x_{\xi}, n}\left(x_{\xi}, y_{\xi}\right)$ and $F_{y_{\xi}, n}\left(x_{\xi}, y_{\xi}\right)$ associated to each access port are defined in the section A.1 of the Appendix A.

In Fig. 4.8, the coordinate system $\left(x_{1}, y_{1}, z_{1}\right)$ is shown. This coordinate system has been used to describe the modal vectors of the parallel access ports $\xi=1$ and $\xi=2$. It is remarkable that, due to this election, the propagative direction of the modes from the port $\xi=1$ points inward the rectangular cavity, whereas in the case of the modes from the port $\xi=2$, it points outward the rectangular cavity. Therefore, we must introduce a negative sign in the expression of the modal vectors at the port $\xi=2$ in order to use them properly. This is due to the fact that the magnetic modal vectors are related to the equivalent modal currents that characterize the GAM [107], and they are always defined in the inward direction to the access. Furthermore, in the figure 4.8 the coordinate systems $\left(x_{3}, y_{3}, z_{3}\right)$ and $\left(x_{5}, y_{5}, z_{5}\right)$ are also shown. The former is used to describe the modal vectors of the parallel ports $\xi=3$ and $\xi=4$. In this case the modal vectors of port $\xi=4$ must be also modified with a minus sign, 
since we have used the coordinate system $\left(x_{3}, y_{3}, z_{3}\right)$ to formulate them. The last one is used to formulate the modal vectors of the port $\xi=5$.

The dimensions $l_{x_{\xi}} \times l_{y_{\xi}}$ that define the cross section of each rectangular access port $(\xi)$ have been chosen as follows:

$$
\begin{array}{cll}
l_{x_{1}}=l_{x_{2}}=a ; & l_{x_{3}}=l_{x_{4}}=c ; & l_{x_{5}}=c ; \\
l_{y_{1}}=l_{y_{2}}=b ; & l_{y_{3}}=l_{y_{4}}=b ; & l_{y_{5}}=a
\end{array}
$$

As well as, the dimensions $l_{z_{\xi}}$ related to the propagative direction at each access port are:

$$
l_{z_{1}}=l_{z_{2}}=c ; \quad l_{z_{3}}=l_{z_{4}}=a ; \quad l_{z_{5}}=b
$$

Moreover, we recall that in order to calculate the $\mathbf{S}$ matrix, for convenience, we have formulated the electric modes of the rectangular cavity in the coordinate system $\left(x_{c}, y_{c}, z_{c}\right)$ shown in figure FIGURA. Therefore, to evaluate the integrals involved in the computation of the $\mathbf{F}$ matrix, we have to be coherent and to use the same coordinate system to formulate the magnetic modes of the rectangular resonator. The dimensions of the cavity in this coordinate system are:

$$
l_{x_{c}}=a ; \quad l_{y_{c}}=c ; \quad l_{z_{c}}=b
$$

At this point, we can observe that the expressions (4.146)-(4.148) contain the scalar and dyadic Green's functions which are singular when $\vec{r}=\vec{r}^{\prime}$. Thus, a first inspection could suggest us to proceed like for the $\mathbf{L}$ and $\mathbf{C}$ matrices, extracting the singularities in closed form and transforming the singular integrals to regular ones. Nevertheless, for the computation of the $\mathbf{G}, \mathbf{T}$ and $\mathbf{W}$ matrices we will use a different procedure. The scalar magnetic Green's function, $g^{m}\left(\vec{r}, \vec{r}^{\prime}\right)$, of a rectangular resonator can be expressed as a series of the scalar functions associated to the modal vectors of a rectangular waveguide with the same cross section as the cavity (see equation (A.29) of the Appendix A). This kind of expression does converge very slowly, and for this reason it is usually not convenient to use them for the computations. However, in our case its use results very advantageous, since it allows us to solve analytically the integrals involved in the computation of the $\mathbf{G}$ matrix, as we detail in the next subsections. Analogously, the static dyadic Green's function and its curl, $\underline{G}_{o}^{F}\left(\vec{r}, \vec{r}^{\prime}\right)$ and $\nabla \times \underline{G}_{o}^{F}\left(\vec{r}, \vec{r}^{\prime}\right)$, can be expressed in terms of the modal vectors associated with the access ports (see section A.3 of the Appendix A). Despite the fact that these series are slowly convergent, they permits us to obtain analytical expressions for the integrals involved in the computation of the $\mathbf{T}$ and $\mathbf{W}$ matrices.

\section{A. Computation of the $\mathbf{G}$ matrix}

The expression of the entries $G_{m n}^{(\gamma, \xi)}$ is:

$$
G_{m n}^{(\gamma, \xi)}=\int_{S_{\gamma}} \int_{S_{\xi}} \nabla_{s} \cdot \vec{h}_{m}^{(\gamma)}(\vec{r}) g^{m}\left(\vec{r}, \vec{r}^{\prime}\right) \nabla_{s}^{\prime} \cdot \vec{h}_{n}^{(\xi)}\left(\vec{r}^{\prime}\right) d s d s^{\prime}
$$


To evaluate this integral, firstly we calculate the divergence of the magnetic modal vector at the access port $(\xi)$ (see expressions (A.11) y (A.13) of the Appendix A):

$$
\nabla_{s}^{\prime} \cdot \vec{h}_{n}^{(\xi)}\left(\vec{r}^{\prime}\right)=\left\{\begin{array}{cc}
\nu_{\xi} k_{t, n}^{(\xi)} \phi_{n_{x_{\xi}}, n_{y_{\xi}}}^{(\xi) \mathrm{TE}}\left(x_{\xi}, y_{\xi}\right), & \text { if } n \text { is a TE} \\
0, & \text { if } n \text { is a } \mathrm{TM}^{z_{\xi}} \text { mode }
\end{array}\right.
$$

where $\left(n_{x_{\xi}}, n_{y_{\xi}}\right)$ are the modal indexes associated to the $n$-th mode at the port $(\xi)$ which are related to the coordinates $x_{\xi}$ y $y_{\xi}$, respectively; $k_{t, n}^{(\xi)}$ is the $n$-th cutoff wavenumber at the same port; $\phi_{n_{x_{\xi}}, n_{y_{\xi}}}^{(\xi) \mathrm{TE}}\left(x_{\xi}, y_{\xi}\right)$ is the scalar modal function used to obtain the modal eigenvectors $\mathrm{TE}^{z_{\xi}}$, which is defined by the equation (A.10) of the Appendix A; and $\nu_{\xi}$ is a constant factor that forces the propagative direction to point inward to the rectangular cavity. In particular:

$$
\nu_{\xi}=\left\{\begin{array}{l}
1, \quad \text { if } \xi=1,3,5 \\
-1, \text { if } \xi=2,4
\end{array}\right.
$$

Therefore, it is easily observed that if any of the magnetic modes $\vec{h}_{m}^{(\gamma)}(\vec{r})$ or $\vec{h}_{n}^{(\xi)}\left(\vec{r}^{\prime}\right)$ are $\mathrm{TM}^{z_{\xi}}$ modes, then:

$$
G_{m n}^{(\gamma, \xi)}=0
$$

Thus, to evaluate the expression (4.154) when both $\vec{h}_{m}^{(\gamma)}(\vec{r})$ and $\vec{h}_{n}^{(\xi)}\left(\vec{r}^{\prime}\right)$ are $\mathrm{TE}^{z_{\xi}}$ modes, we define the following surface integral:

$$
\zeta_{n}^{(\xi)}(\mathbf{r}) \equiv \int_{S_{\xi}} g^{m}\left(\vec{r}, \vec{r}^{\prime}\right) \nabla_{s}^{\prime} \cdot \vec{h}_{n}^{(\xi)}\left(\vec{r}^{\prime}\right) d s^{\prime}
$$

Introducing (4.155) and (A.29) in this expression, we have:

$$
\zeta_{n}^{(\xi)}(\mathbf{r})=\nu_{\xi} \frac{k_{t, n}^{(\xi)}}{l_{z_{\xi}}} \phi_{n_{x_{\xi}}, n_{y_{\xi}}}^{(\xi) \mathrm{TE}}\left(x_{\xi}, y_{\xi}\right) \sum_{n_{z_{\xi}}=0}^{\infty} \nu_{\xi}^{n_{z_{\xi}}} \epsilon_{n_{z_{\xi}}} \frac{\cos \left(k_{z_{\xi}} z_{\xi}\right)}{\left(k_{t, n}^{(\xi)}\right)^{2}+k_{z_{\xi}}^{2}}
$$

where $\epsilon_{n}$ is the Neumman factor (eq. (A.6)) and $k_{z_{\xi}}$ is the wavenumber of the rectangular cavity related to the $z_{\xi}$ coordinate defined by the equation (A.31).

Therefore, if $m$ is a mode of the access port $(\gamma)$ and $\left(m_{x_{\gamma}}, m_{y_{\gamma}}\right)$ are the modal indexes related to the coordinates $x_{\gamma}$ and $y_{\gamma}$, respectively, then the entry $G_{m, n}^{(\gamma, \xi)}$ can be expressed as follows:

$$
\begin{array}{r}
G_{m n}^{(\gamma, \xi)}=\int_{S_{\gamma}} \nabla_{S} \cdot \mathbf{h}_{m}^{(\gamma)}(\mathbf{r}) \zeta_{n}^{(\xi)}(\mathbf{r}) d S=\frac{1}{l_{z_{\xi}}} \nu_{\gamma} \nu_{\xi} k_{t, m}^{(\gamma)} k_{t, n}^{(\xi)} \sum_{n_{z_{\xi}}=0}^{\infty} \nu_{\xi}^{n_{z_{\xi}}} \frac{\epsilon_{n_{z_{\xi}}}}{\left(k_{t, n}^{(\xi)}\right)^{2}+k_{z_{\xi}}^{2}} \\
\cdot \int_{S_{\gamma}} \phi_{m_{x_{\gamma}}, m_{y_{\gamma}}}^{(\gamma) \mathrm{TE}}\left(x_{\gamma}, y_{\gamma}\right) \phi_{n_{x_{\xi}}, n_{y_{\xi}}}^{(\xi) \mathrm{TE}}\left(x_{\xi}, y_{\xi}\right) \cos \left(k_{z_{\xi}} z_{\xi}\right) d S
\end{array}
$$


where $\phi_{m_{x \gamma}, m_{y_{\gamma}}}^{(\gamma) \mathrm{TE}}\left(x_{\gamma}, y_{\gamma}\right)$ represents the potential function of the $m$-th $\mathrm{TE}^{z_{\gamma}}$ mode of the port $(\gamma)$, and $k_{t, m}^{(\gamma)}$ is the cutoff wavenumber of that mode.

In order to evaluate the integral (4.160), we need to express its integrand in one coordinate system. For this purpose, we reformulate the potential function $\phi_{m_{x_{\gamma}}, m_{y_{\gamma}}}^{(\gamma) \mathrm{TE}}\left(x_{\gamma}, y_{\gamma}\right)$ in the coordinate system $\left(x_{\xi}, y_{\xi}, z_{\xi}\right)$. Furthermore, it is convenient to notice that the integral must be evaluated in the rectangular cross section defined by the port $(\gamma)$. This surface is described by the plane $\vartheta_{\xi}=$ const, whit $\vartheta=\left(x_{\xi}, y_{\xi}, z_{\xi}\right)$. For instance, the surface related to port (1) is on the plane $x_{3}=$ const and the plane $x_{5}=$ const. Thus, the surface integral above can always be expressed as two unidimensional integrals of the kind:

$$
\int_{0}^{l_{\varrho_{\xi}}} \cos \left(k_{\varrho_{\xi}, m} \varrho_{\xi}\right) \cos \left(k_{\varrho_{\xi}, n} \varrho_{\xi}\right) d \varrho_{\xi}=\frac{l_{\varrho_{\xi}}}{\epsilon_{n_{\varrho_{\xi}}}} \delta_{m_{\varrho_{\xi}}, n_{\varrho_{\xi}}}, \quad \text { with } \varrho=\left(x_{\xi}, y_{\xi}, z_{\xi}\right) \neq \vartheta_{\xi}
$$

where $m_{\varrho_{\xi}}$ is the modal index of the $m$-th mode related to the coordinate $\varrho_{\xi} ; k_{\varrho_{\xi}, m}$ is the cutoff wavenumber related to the coordinate $\varrho_{\xi}$ which is defined as $k_{\varrho_{\xi}, m}=m_{\varrho_{\xi}} \pi / l_{\varrho_{\xi}}$, and $\delta_{m, n}$ is the Kronecker delta function.

At this point, by solving analytically the surface integral in (4.160) by means of (4.161), we obtain as a result an infinite series that can be also solved analytically, as it is detailed in the section F.1 of the Appendix F.

Using this procedure, the entries of the $G_{m n}^{(\gamma, \xi)}$ matrix which relate parallel access ports, this is, the entries $G_{m n}^{(\xi, \xi)}$ with $\xi=1,2, \ldots, 5$ and the entries $G_{m n}^{(\xi, \xi+1)}$ with $\xi=1,3$, can be expressed as:

$$
\begin{aligned}
G_{m n}^{(\xi, \xi)} & =k_{t, n}^{(\xi)} \operatorname{coth}\left(k_{t, n}^{(\xi)} l_{z_{\xi}}\right) \delta_{m, n} \\
G_{m n}^{(\xi, \xi+1)} & =-\frac{k_{t, n}^{(\xi)}}{\sinh \left(k_{t, n}^{(\xi)} l_{z_{\xi}}\right)} \delta_{m, n}
\end{aligned}
$$

And the entries of the matrix blocks that relate the orthogonal access ports, this is, the elements $G_{m n}^{(\gamma, \xi)}$ with $\gamma=1,2$ and $\xi=3,4,5$, or with $\gamma=3,4$ and $\xi=5$, are given by,

$$
G_{m n}^{(\gamma, \xi)}=\frac{k_{t, m}^{(\gamma)} k_{t, n}^{(\xi)}}{\left(k_{t, n}^{(\xi)}\right)^{2}+k_{m_{z_{\xi}}}^{(\gamma) 2}} \frac{\nu_{\gamma} \nu_{\xi}}{\sqrt{l_{z_{\xi}} l_{z_{\gamma}}}} \rho_{m, n}^{(\gamma, \xi)} \psi_{m, n}^{(\gamma, \xi)}
$$

where $k_{m_{z_{\xi}}}^{(\gamma)}$ represents the wavenumber of the $m$-th mode of the port $(\gamma)$ associated to the coordinate $z_{\xi}$; and $\rho_{m, n}^{(\gamma, \xi)}$ and $\psi_{m, n}^{(\gamma, \xi)}$ are auxiliary functions which definition can be found in section F.2 of the Appendix F.

Finally, it is remarkable that, since the matrix $\mathbf{G}$ is symmetric, we have only evaluated 
the matrix blocks $G_{m n}^{(\gamma, \xi)}$ with $\xi \geq \gamma$.

\section{B. Computation of the T matrix}

As it has been reported before, the blocks of the $\mathbf{T}$ matrix, $T_{m n}^{(\gamma, \xi)}$, are:

$$
\left.T_{m n}^{(\gamma, \xi}\right)=\int_{S_{\gamma}} \int_{S_{\xi}} \vec{h}_{m}^{(\gamma)}(\vec{r}) \cdot \underline{G}_{o}^{F}\left(\vec{r}, \vec{r}^{\prime}\right) \cdot \vec{h}_{n}^{(\xi)}\left(\vec{r}^{\prime}\right) d s d s^{\prime}
$$

where the expression for the static part of the dyadic Green's function for the electric potential vector of a rectangular resonator, $\underline{G}_{o}^{F}\left(\vec{r}, \vec{r}^{\prime}\right)$, can be found in the equation (A.33) of the Appendix A. To evaluate this integral, we will assume that $m$ represents a mode of the port $(\gamma)$, being $\left(m_{x_{\gamma}}, m_{y_{\gamma}}\right)$ its modal indexes related to the coordinates $x_{\gamma}$ e $y_{\gamma}$, respectively. Analogously, we will also assume that $n$ is a mode of the port $(\xi)$, and its modal indexes $\left(n_{x_{\xi}}, n_{y_{\xi}}\right)$ are related to the transverse coordinates $x_{\xi}$ e $y_{\xi}$, respectively.

In order to evaluate the entries of the $\mathbf{T}$ matrix, we will first consider the following vector surface integral:

$$
\vec{\Gamma}_{n}^{(\xi)}(\vec{r})=\int_{S_{\xi}} \underline{G}_{o}^{F}\left(\vec{r}, \vec{r}^{\prime}\right) \cdot \vec{h}_{n}^{(\xi)}\left(\vec{r}^{\prime}\right) d s^{\prime}=\Gamma_{x_{\xi}, n}^{(\xi)} \hat{\mathbf{x}}_{\xi}+\Gamma_{y_{\xi}, n}^{(\xi)} \hat{\mathbf{y}}_{\xi}+\Gamma_{z_{\xi}, n}^{(\xi)} \hat{\mathbf{z}}_{\xi}
$$

where the three components of the vector $\vec{\Gamma}_{n}^{(\xi)}(\vec{r})$ can be expressed as:

$$
\begin{aligned}
& \Gamma_{x_{\xi}, n}^{(\xi)}=\int_{S_{\xi}}\left(h_{x_{\xi}^{\prime}, n}^{(\xi)} G_{0, x_{\xi} x_{\xi}^{\prime}}^{\mathrm{F}}+h_{y_{\xi}^{\prime}, n}^{(\xi)} G_{0, x_{\xi} y_{\xi}^{\prime}}^{\mathrm{F}}+h_{z_{\xi}^{\prime}, n}^{(\xi)} G_{0, x_{\xi} z_{\xi}^{\prime}}^{\mathrm{F}}\right) d s^{\prime} \\
& \Gamma_{y_{\xi}, n}^{(\xi)}=\int_{S_{\xi}}\left(h_{x_{\xi}^{\prime}, n}^{(\xi)} G_{0, y_{\xi} x_{\xi}^{\prime}}^{\mathrm{F}}+h_{y_{\xi}^{\prime}, n}^{(\xi)} G_{0, y_{\xi} y_{\xi}^{\prime}}^{\mathrm{F}}+h_{z_{\xi}^{\prime}, n}^{(\xi)} G_{0, y_{\xi} z_{\xi}^{\prime}}^{\mathrm{F}}\right) d s^{\prime} \\
& \Gamma_{z_{\xi}, n}^{(\xi)}=\int_{S_{\xi}}\left(h_{x_{\xi}^{\prime}, n}^{(\xi)} G_{0, z_{\xi} x_{\xi}^{\prime}}^{\mathrm{F}}+h_{y_{\xi}^{\prime}, n}^{(\xi)} G_{0, z_{\xi} y_{\xi}^{\prime}}^{\mathrm{F}}+h_{z_{\xi}^{\prime}, n}^{(\xi)} G_{0, z_{\xi} z_{\xi}^{\prime}}^{\mathrm{F}}\right) d s^{\prime}
\end{aligned}
$$

Thus, introducing the modal vector functions of the rectangular access ports and the dyadic Green's function (see Appendix A) in the equations (4.167), we can obtain the value of the vector $\vec{\Gamma}_{n}^{(\xi)}(\vec{r})$ in the case that the mode $n$ is a $\mathrm{TE}^{z_{\xi}}$ mode:

$$
\begin{aligned}
\Gamma_{x_{\xi}, n_{\mathrm{TE}}}^{(\xi)} & =\nu_{\xi} \frac{2}{l_{z_{\xi}}} N_{x_{\xi}, n}^{\mathrm{TE}} \sin \left(k_{x_{\xi}, n} x_{\xi}\right) \cos \left(k_{y_{\xi}, n} y_{\xi}\right) \Theta_{n_{\mathrm{TE}}}^{(\xi)}\left(z_{\xi}\right) \\
\Gamma_{y_{\xi}, n_{\mathrm{TE}}}^{(\xi)} & =\nu_{\xi} \frac{2}{l_{z_{\xi}}} N_{y_{\xi}, n}^{\mathrm{TE}} \cos \left(k_{x_{\xi}, n} x_{\xi}\right) \sin \left(k_{y_{\xi}, n} y_{\xi}\right) \Theta_{n_{\mathrm{TE}}}^{(\xi)}\left(z_{\xi}\right) \\
\Gamma_{z_{\xi}, n_{\mathrm{TE}}}^{(\xi) \mathrm{TE}} & =-\nu_{\xi} \frac{2}{l_{z_{\xi}}} N_{x_{\xi}, n}^{\mathrm{TE}} \frac{\left(k_{t, n}^{(\xi)}\right)^{2}}{k_{x_{\xi}, n}} \cos \left(k_{x_{\xi}, n} x_{\xi}\right) \cos \left(k_{y_{\xi}, n} y_{\xi}\right) \Phi_{n_{\mathrm{TE}}}^{(\xi)}\left(z_{\xi}\right)
\end{aligned}
$$


And the value of $\vec{\Gamma}_{n}^{(\xi)}(\vec{r})$ when the mode $n$ is a $\mathrm{TM}^{z_{\xi}}$ mode:

$$
\begin{aligned}
\Gamma_{x_{\xi}, n_{\mathrm{TM}}}^{(\xi)} & =\nu_{\xi} \frac{1}{l_{z_{\xi}}} N_{x_{\xi}, n}^{\mathrm{TM}} \sin \left(k_{x_{\xi}, n} x_{\xi}\right) \cos \left(k_{y_{\xi}, n} y_{\xi}\right) \Theta_{n_{\mathrm{TM}}}^{(\xi)}\left(z_{\xi}\right) \\
\Gamma_{y_{\xi}, n_{\mathrm{TM}}}^{(\xi)} & =-\nu_{\xi} \frac{1}{l_{z_{\xi}}} N_{y_{\xi}, n}^{\mathrm{TM}} \cos \left(k_{x_{\xi}, n} x_{\xi}\right) \sin \left(k_{y_{\xi}, n} y_{\xi}\right) \Theta_{n_{\mathrm{TM}}}^{(\xi)}\left(z_{\xi}\right) \\
\Gamma_{z_{\xi}, n_{\mathrm{TM}}}^{(\xi)} & =0
\end{aligned}
$$

In the expressions (4.168a)-(4.169c), we have made use of the infinite series:

$$
\begin{aligned}
& \Theta_{n_{\mathrm{TE}}}^{(\xi)}\left(z_{\xi}\right)=\sum_{n_{z_{\xi}}=1}^{\infty} \frac{k_{z_{\xi}}^{2}}{\left(\left(k_{t, n}^{(\xi)}\right)^{2}+k_{z_{\xi}}^{2}\right)^{2}} \cos \left(k_{z_{\xi}} z_{\xi}\right) \nu_{\xi}^{n_{z_{\xi}}} \\
& \Theta_{n_{\mathrm{TM}}}^{(\xi)}\left(z_{\xi}\right)=\sum_{n_{z_{\xi}}=0}^{\infty} \frac{\epsilon_{n_{z_{\xi}}}}{\left(k_{t, n}^{(\xi)}\right)^{2}+k_{z_{\xi}}^{2}} \cos \left(k_{z_{\xi}} z_{\xi}\right) \nu_{\xi}^{n_{z_{\xi}}} \\
& \Phi_{n_{\mathrm{TE}}}^{(\xi)}\left(z_{\xi}\right)=\sum_{n_{z_{\xi}}=1}^{\infty} \frac{k_{z_{\xi}}}{\left(\left(k_{t, n}^{(\xi)}\right)^{2}+k_{z_{\xi}}^{2}\right)^{2}} \sin \left(k_{z_{\xi}} z_{\xi}\right) \nu_{\xi}^{n_{z_{\xi}}}
\end{aligned}
$$

where $k_{z_{\xi}}=n_{z_{\xi}} \pi / l_{z_{\xi}}$.

Consequently, the $T_{m n}^{(\gamma, \xi)}$ matrix can be calculated as follows:

$$
T_{m n}^{(\gamma, \xi)}=\int_{S_{\gamma}} \vec{h}_{m}^{(\gamma)}(\vec{r}) \cdot \vec{\Gamma}_{n}^{(\xi)}(\vec{r}) d s=\int_{S_{\gamma}}\left(h_{x_{\gamma}, m}^{(\gamma)} \Gamma_{x_{\xi}, n}^{(\xi)}+h_{y_{\gamma}, m}^{(\gamma)} \Gamma_{y_{\xi}, n}^{(\xi)}+h_{z_{\gamma}, m}^{(\gamma)} \Gamma_{z_{\xi}, n}^{(\xi)}\right) d s
$$

In order to evaluate the integral (4.173), we proceed analogously to the calculation of (4.160). We express the modal vector $\vec{h}_{m}^{(\gamma)}(\vec{r})$ in the coordinate system $\left(x_{\xi}, y_{\xi}, z_{\xi}\right)$. Furthermore, the integral must be evaluated in the rectangular cross section defined by the port $(\gamma)$. Therefore, the surface is described by the plane $\vartheta_{\xi}=$ const, with $\vartheta=\left(x_{\xi}, y_{\xi}, z_{\xi}\right)$. In this way, the surface integral above can be expressed as unidimensional integrals of the type described in (4.161) or of the type:

$$
\int_{0}^{l_{\varrho_{\xi}}} \sin \left(k_{\varrho_{\xi}, m} \varrho_{\xi}\right) \sin \left(k_{\varrho_{\xi}, n} \varrho_{\xi}\right) d \varrho_{\xi}=\frac{l_{\varrho_{\xi}}}{2}\left(\epsilon_{n_{\varrho_{\xi}}}-1\right) \delta_{m_{\varrho_{\xi}}, n_{\varrho_{\xi}}}, \quad \text { with } \varrho=\left(x_{\xi}, y_{\xi}, z_{\xi}\right) \neq \vartheta_{\xi}
$$

In this way, by solving analytically the surface integral in (4.173) by means of (4.161) and (4.174), we obtain as a result infinite series that can be also summed in a closed form, as it is detailed in the section F.1 of the Appendix F.

Finally, the results obtained for the blocks of the $\mathbf{T}$ matrix which relate parallel access ports, this is, the entries $T_{m n}^{(\xi, \xi)}$ with $\xi=1,2, \ldots, 5$ and the entries $T_{m n}^{(\xi, \xi+1)}$ with $\xi=1,3$. 
Moreover, we must take into account that, in this case, the modes $m$ and $n$ can be TE or TM modes:

$$
\begin{aligned}
& T_{m_{\mathrm{TE}}, n_{\mathrm{TE}}}^{(\xi, \xi)}=\frac{1}{2 k_{t, n}^{(\xi)}}\left[\operatorname{coth}\left(k_{t, n}^{(\xi)} l_{z_{\xi}}\right)-\frac{k_{t, n}^{(\xi)} l_{z_{\xi}}}{\sinh ^{2}\left(k_{t, n}^{(\xi)} l_{z_{\xi}}\right)}\right] \delta_{m, n} \\
& T_{m_{\mathrm{TE}}, n_{\mathrm{TE}}}^{(\xi, \xi+1)}=-\frac{1}{2 k_{t, n}^{(\xi)}} \frac{1}{\sinh \left(k_{t, n}^{(\xi)} l_{z_{\xi}}\right)}\left(1-\frac{k_{t, n}^{(\xi)} l_{z_{\xi}}}{\tanh \left(k_{t, n}^{(\xi)} l_{z_{\xi}}\right)}\right) \delta_{m, n} \\
& T_{m_{\mathrm{TM}}, n_{\mathrm{TE}}}^{(\xi, \xi)}= T_{m_{\mathrm{TE}}, n_{\mathrm{TM}}}^{(\xi, \xi)}=T_{m_{\mathrm{TM}}, n_{\mathrm{TE}}}^{(\xi, \xi+1)}=T_{m_{\mathrm{TE}}, n_{\mathrm{TM}}}^{(\xi, \xi+1)}=0 \\
& T_{m_{\mathrm{TM}}, n_{\mathrm{TM}}}^{(\xi, \xi)}=\frac{1}{k_{t, n}^{(\xi)}} \operatorname{coth}\left(k_{t, n}^{(\xi)} l_{z_{\xi}}\right) \delta_{m, n} \\
& T_{m_{\mathrm{TM}}, n_{\mathrm{TM}}}^{(\xi, \xi+1)}=-\frac{1}{k_{t, n}^{(\xi)}} \frac{1}{\sinh \left(k_{t, n}^{(\xi)} l_{z_{\xi}}\right)} \delta_{m, n}
\end{aligned}
$$

And the elements of the matrix which relate orthogonal access ports, this is, the elements $T_{m n}^{(\gamma, \delta)}$ with $\gamma=1,2 \mathrm{y} \xi=3,4,5$; or with $\gamma=3,4$ y $\xi=5$, are given by:

$$
\begin{aligned}
& T_{m_{\mathrm{TE}}, n_{\mathrm{TE}}}^{(\gamma, \xi)}=\nu_{\gamma} \nu_{\xi} \frac{k_{m_{x_{\gamma}}}^{2}}{k_{t, m}^{(\gamma)}} \frac{\rho_{m, n}^{(\gamma, \xi)} \psi_{m, n}^{(\gamma, \xi)}}{\left(\left(k_{t, n}^{(\xi)}\right)^{2}+k_{m_{z_{\xi}}}^{2}\right)^{2}} \frac{1}{\sqrt{l_{z_{\gamma}} l_{z_{\xi}}}}\left[\frac{1}{k_{t, n}^{(\xi)}} \chi_{m, n}^{(\gamma, \xi)}-k_{t, n}^{(\xi)} \varphi_{m, n}^{(\gamma, \xi)}\right](4 \\
& T_{m_{\mathrm{TM}}, n_{\mathrm{TE}}}^{(\gamma, \xi)}=-\nu_{\gamma} \nu_{\xi} \frac{k_{m_{x_{\gamma}}}}{k_{t, m}^{(\gamma)}} \frac{\zeta_{m, n}^{(\gamma, \xi)} \tau^{(\gamma, \xi)}}{\left(\left(k_{t, n}^{(\xi)}\right)^{2}+k_{m_{z_{\xi}}}^{2}\right)^{2}} \sqrt{\frac{2}{l_{z_{\gamma}} l_{z_{\xi}}}}\left[k_{t, n}^{(\xi)}+\frac{k_{m_{x_{\gamma}}}^{2}}{k_{t, n}^{(\xi)}}\right] \psi_{m, n}^{(\gamma, \xi)} \\
& T_{m_{\mathrm{TE}}, n_{\mathrm{TM}}}^{(\gamma, \xi)}=-\frac{k_{n_{x_{\xi}}} k_{n_{y_{\xi}}}}{k_{t, m}^{(\gamma)} k_{t, n}^{(\xi)}} \frac{\nu_{\gamma} \nu_{\xi} \theta_{m, n}^{(\gamma, \xi)}}{\left(k_{t, n}^{(\xi)}\right)^{2}+k_{m_{z_{\xi}}}^{2}} \sqrt{\frac{2}{l_{z_{\gamma}} l_{z_{\xi}}}} \psi_{m, n}^{(\gamma, \xi)} \\
& T_{m_{\mathrm{TM}}, n_{\mathrm{TM}}}^{(\gamma, \xi)}=\frac{k_{m_{x_{\gamma}}}}{k_{t, m}^{(\gamma)} k_{t, n}^{(\xi)}} \frac{\nu_{\gamma} \nu_{\xi} \lambda_{m, n}^{(\gamma, \xi)}}{\left(k_{t, n}^{(\xi)}\right)^{2}+k_{m_{z_{\xi}}}^{2}} \frac{2}{\sqrt{l_{z_{\gamma}} l_{z_{\xi}}}} \tau^{(\gamma, \xi)} \psi_{m, n}^{(\gamma, \xi)}
\end{aligned}
$$

where the next auxiliary functions $\chi_{m, n}^{(\gamma, \xi)}, \varphi_{m, n}^{(\gamma, \xi)}, \zeta_{m, n}^{(\gamma, \xi)}, \tau^{(\gamma, \xi)}, \theta_{m, n}^{(\gamma, \xi)}$ and $\lambda_{m, n}^{(\gamma, \xi)}$ have been defined in the section F.2 of the Appendix F. To conclude, it is remarkable that the $\mathbf{T}$ matrix is also symmetric. For this reason, we have only presented the expressions of $\mathbf{T}^{(\gamma, \xi)}$ for the cases $\xi \geq \gamma$.

\section{Computation of the W matrix}

The $\mathbf{W}$ matrix can be also calculated by blocks, $W_{m n}^{(\xi)}$, which expression is:

$$
W_{m n}^{(\xi)}=\int_{V_{1}} \int_{S_{\xi}} \vec{w}_{m}(\vec{r}) \cdot \nabla \times \underline{G}_{o}^{F}\left(\vec{r}, \vec{r}^{\prime}\right) \cdot \vec{h}_{n}^{(\xi)}\left(\vec{r}^{\prime}\right) d s^{\prime} d v
$$


where the expression of the curl of the dyadic Green's function for the electric vector potential of a rectangular cavity can be found in the equation (A.36) of the Appendix A. Firstly, in order to calculate this integral, let us consider the following surface integral in the primed coordinate system:

$$
\vec{\Psi}_{n}^{(\xi)}(\vec{r}) \equiv \int_{S_{\xi}} \nabla \times \underline{G}_{o}^{F}\left(\vec{r}, \vec{r}^{\prime}\right) \cdot \vec{h}_{n}^{(\xi)}\left(\vec{r}^{\prime}\right) d s^{\prime}
$$

We substitute now the equations (A.34)-(A.35) and (A.37)-(A.38) into (A.36) to obtain the expression of $\underline{G}_{o}^{F}\left(\vec{r}, \vec{r}^{\prime}\right)$ in terms of the magnetic modal vectors of the access ports. Thus, we can evaluate (4.185) taking into account wether the $n$ mode of the access port is a TE ${ }^{z_{\xi}}$ mode or a $\mathrm{TM}^{z_{\xi}}$ one:

$$
\begin{aligned}
\vec{\Psi}_{n_{\mathrm{TE}}}^{(\xi)}(\vec{r})= & \frac{\epsilon_{n_{x_{\xi}}} \epsilon_{n_{y_{\xi}}}}{\sqrt{l_{x_{\xi}} l_{y_{\xi}}}} \frac{1}{k_{t, n}^{(\xi)}} \frac{\sinh \left(k_{t, n}^{(\xi)} \Upsilon^{(\xi)}\left(z_{\xi}\right)\right)}{\sinh \left(k_{t, n}^{(\xi)} l_{z_{\xi}}\right)}\left\{k_{y_{\xi}, n} \cos \left(k_{x_{\xi}, n} x_{\xi}\right) \sin \left(k_{y_{\xi}, n} y_{\xi}\right) \hat{\mathbf{x}}_{\xi}\right. \\
& \left.-k_{x_{\xi}, n} \sin \left(k_{x_{\xi}, n} x_{\xi}\right) \cos \left(k_{y_{\xi}, n} y_{\xi}\right) \hat{\mathbf{y}}_{\xi}\right\} \\
\vec{\Psi}_{n_{\mathrm{TM}}}^{(\xi)}(\vec{r})= & -\frac{2}{\sqrt{l_{x_{\xi}} l_{y_{\xi}}}} \frac{1}{k_{t, n}^{(\xi)}} \frac{\sinh \left(k_{t, n}^{(\xi)} \Upsilon^{(\xi)}\left(z_{\xi}\right)\right)}{\sinh \left(k_{t, n}^{(\xi)} l_{z_{\xi}}\right)}\left\{k_{x_{\xi}, n} \cos \left(k_{x_{\xi}, n} x_{\xi}\right) \sin \left(k_{y_{\xi}, n} y_{\xi}\right) \hat{\mathbf{x}}_{\xi}\right. \\
& \left.+k_{y_{\xi}, n} \sin \left(k_{x_{\xi}, n} x_{\xi}\right) \cos \left(k_{y_{\xi}, n} y_{\xi}\right) \hat{\mathbf{y}}_{\xi}\right\} \\
& +\nu_{\xi} \frac{2}{\sqrt{l_{x_{\xi}} l_{y_{\xi}}}} \sin \left(k_{x_{\xi}, n} x_{\xi}\right) \sin \left(k_{y_{\xi}, n} y_{\xi}\right) \frac{\cosh \left(k_{t, n}^{(\xi)} \Upsilon^{(\xi)}\left(z_{\xi}\right)\right)}{\sinh \left(k_{t, n}^{(\xi)} l_{z_{\xi}}\right)} \hat{\mathbf{z}}_{\xi}
\end{aligned}
$$

where the following auxiliary function has been defined:

$$
\Upsilon^{(\xi)}\left(z_{\xi}\right) \equiv\left\{\begin{array}{l}
l_{z_{\xi}}-z_{\xi}, \text { if } \xi=1,3,5 \\
z_{\xi}, \text { if } \xi=2,4
\end{array}\right.
$$

Therefore, the entries of the $W_{m n}^{(\xi)}$ matrix can be finally calculated as:

$$
W_{m n}^{(\xi)}=\int_{V_{1}} \vec{w}_{m}(\vec{r}) \cdot \vec{\Psi}_{n}^{(\xi)}(\mathbf{r}) d v
$$

This last integral can be performed numerically. For this purpose, to compute any block matrix $\mathbf{W}^{(\xi)}$ we must express the basis functions $\vec{w}_{m}(\vec{r})$ in the coordinate system $\left(x_{\xi}, y_{\xi}, z_{\xi}\right)$ of each port $(\xi)$. As a consequence, we are able to calculate the elements of the $\mathbf{W}$ matrix in terms of 3-D integrals, instead of solving 5-D ones. Note that these integrals do not present singularities.

\section{Computation of the F matrix}

The entries of the $\mathbf{F}$ matrix are:

$$
F_{m n}^{(\xi)}=\int_{S} \vec{H}_{m}(\vec{r}) \cdot \vec{h}_{n}^{(\xi)}(\vec{r}) d s
$$


where $\vec{H}_{m}(\vec{r})$ is the magnetic field associated to the $m$-th mode of the rectangular cavity. To evaluate the expression of the entries $F_{m n}^{(\xi)}$, we must express the integrand in one coordinate system. For this purpose, we will reference the magnetic modal vector $\vec{H}_{m}(\vec{r})$, formulated in the coordinate system $\left(x_{c}, y_{c}, z_{c}\right)$ (see Fig. 4.7), to the coordinate systems $\left(x_{\xi}, y_{\xi}, z_{\xi}\right)$ of each access port $(\xi)$.

Now, let us consider that $\left(m_{x_{c}}, m_{y_{c}}, m_{z_{c}}\right)$ represent the modal indexes of to the $m$-th mode of the rectangular cavity, related to the coordinates $\left(x_{c}, y_{c}, z_{c}\right)$, respectively; and $\left(n_{x_{\xi}}, n_{y_{\xi}}\right)$ are the modal indexes of the $n$-th mode of the access port, related to the coordinates $x_{\xi} \mathrm{e} y_{\xi}$, respectively. Using now the expressions (A.20) and (A.27) of Appendix A for the magnetic modal vectors $\mathrm{TE}^{z_{c}}$ and $\mathrm{TM}^{z_{c}}$ of a rectangular cavity, we can obtain the value of $F_{m n}^{(\xi)}$ for $\xi=1,2$ :

$$
\begin{aligned}
F_{m_{\mathrm{TE}}, n_{\mathrm{TE}}}^{(\xi)}= & \nu_{\xi}^{m_{y_{c}}+1} \frac{\sqrt{\epsilon_{m_{x_{c}}} \epsilon_{m_{y_{c}}} \epsilon_{n_{x_{\xi}}} \epsilon_{n_{y_{\xi}}}}}{k_{m} k_{t, n}^{(\xi)} \sqrt{2 l_{y_{c}}}}(-1)^{m_{x_{c}}}\left[\frac{k_{x_{c}, m} k_{z_{c}, m} k_{x_{\xi}, n}}{2 k_{t, m}}\left(\epsilon_{m_{x_{c}}}-1\right)\right. \\
& \left.-\frac{k_{t, m} k_{y_{\xi}, n}}{\epsilon_{m_{x_{c}}}}\right] \delta_{m_{x_{c}}, n_{x_{\xi}}} \delta_{m_{z_{c}}, n_{y_{\xi}}} \\
F_{m_{\mathrm{TE}}, n_{\mathrm{TM}}}^{(\xi)}= & \nu_{\xi}^{m_{y_{c}}+1} \frac{\sqrt{\epsilon_{m_{x_{c}}} \epsilon_{m_{y_{c}}}}}{k_{m} k_{t, n}^{(\xi)} \sqrt{2 l_{y_{c}}}}(-1)^{m_{x_{c}}}\left[\frac{k_{x_{c}, m} k_{z_{c}, m} k_{y_{\xi}, n}}{k_{t, m}}\right. \\
& \left.+k_{t, m} k_{x_{\xi}, n}\right] \delta_{m_{x_{c}}, n_{x_{\xi}}} \delta_{m_{z_{c}}, n_{y_{\xi}}} \\
F_{m_{\mathrm{TM}}, n_{\mathrm{TE}}}^{(\xi)}= & \nu_{\xi}^{m_{y_{c}}+1} \sqrt{\frac{\epsilon_{m_{z_{c}}} \epsilon_{n_{y_{\xi}}}}{\epsilon_{n_{x_{\xi}}} l_{y_{c}}}} \frac{k_{y_{c}, m} k_{x_{\xi}, n}}{k_{t, m} k_{t, n}^{(\xi)}}(-1)^{m_{x_{c}}} \delta_{m_{x_{c}}, n_{x_{\xi}}} \delta_{m_{z_{c}}, n_{y_{\xi}}} \\
F_{m_{\mathrm{TM}}, n_{\mathrm{TM}}}^{(\xi)}= & \nu_{\xi}^{m_{y_{c}}+1} \frac{k_{y_{c}, m} k_{y_{\xi}, n}}{k_{t, m} k_{t, n}^{(\xi)}} \sqrt{\frac{\epsilon_{m_{z_{c}}}}{l_{y_{c}}}}(-1)^{m_{x_{c}}} \delta_{m_{x_{c}}, n_{x_{\xi}}} \delta_{m_{z_{c}}, n_{y_{\xi}}}
\end{aligned}
$$

where $\kappa_{m}$ is the $m$-th resonance wavenumber of the rectangular cavity; $k_{\vartheta_{c}, m}$ is the wavenumber associated with the $m$-th resonant mode of the rectangular cavity, related to the coordinate $\vartheta_{c}$, with $\vartheta_{c}=x_{c}, y_{c}, z_{c} ; k_{t, m}=\sqrt{k_{x_{c}, m}^{2}+k_{y_{c}, m}^{2}}$ is the transverse wavenumber associated 
with the $m$-th resonant mode of the rectangular cavity (see eq. (A.28) Appendix A).

When the access ports are $\xi=3,4$, the expressions for the entries $F_{m n}^{(\xi)}$ are:

$$
\begin{aligned}
& F_{m_{\mathrm{TE}}, n_{\mathrm{TE}}}^{(\xi)}=\nu_{\xi}^{m_{x_{c}}+1} \frac{\sqrt{\epsilon_{m_{x_{c}}} \epsilon_{m_{y_{c}}} \epsilon_{n_{x_{\xi}}} \epsilon_{n_{y_{\xi}}}}}{k_{m} k_{t, n}^{(\xi)} \sqrt{2 l_{x_{c}}}}(-1)^{m_{x_{c}}+m_{y_{c}}}\left[\frac{k_{y_{c}, m} k_{z_{c}, m} k_{x_{\xi}, n}}{2 k_{t, m}}\left(\epsilon_{m_{y_{c}}}-1\right)\right. \\
& \left.-\frac{k_{t, m} k_{y_{\xi}, n}}{\epsilon_{m_{y_{c}}}}\right] \delta_{m_{y_{c}}, n_{x_{\xi}}} \delta_{m_{z_{c}}, n_{y_{\xi}}} \\
& F_{m_{\mathrm{TE}}, n_{\mathrm{TM}}}^{(\xi)}=\nu_{\xi}^{m_{x_{c}}+1} \frac{\sqrt{\epsilon_{m_{x_{c}}} \epsilon_{m_{y_{c}}}}}{k_{m} k_{t, n}^{(\xi)} \sqrt{2 l_{x_{c}}}}(-1)^{m_{x_{c}}+m_{y_{c}}}\left[\frac{k_{y_{c}, m} k_{z_{c}, m} k_{y_{\xi}, n}}{k_{t, m}}\right. \\
& \left.+k_{t, m} k_{x_{\xi}, n}\right] \delta_{m_{y_{c}}, n_{x_{\xi}}} \delta_{m_{z_{c}}, n_{y_{\xi}}} \\
& F_{m_{\mathrm{TM}}, n_{\mathrm{TE}}}^{(\xi)}=\nu_{\xi}^{m_{x_{c}}+1} \sqrt{\frac{\epsilon_{m_{z_{c}}} \epsilon_{n_{x_{\xi}}}}{\epsilon_{n_{y_{\xi}}} l_{x_{c}}}} \frac{k_{x_{c}, m} k_{x_{\xi}, n}}{k_{t, m} k_{t, n}^{(\xi)}}(-1)^{m_{x_{c}}+m_{y_{c}}+1} \delta_{m_{y_{c}}, n_{x_{\xi}}} \delta_{m_{z_{c}}, n_{y_{\xi}}} \\
& F_{m_{\mathrm{TM}}, n_{\mathrm{TM}}}^{(\xi)}=\nu_{\xi}^{m_{x_{c}}+1} \sqrt{\frac{\epsilon_{m_{z_{c}}}}{l_{x_{c}}}} \frac{k_{x_{c}, m} k_{y_{\xi}, n}}{k_{t, m} k_{t, n}^{(\xi)}}(-1)^{m_{x_{c}}+m_{y_{c}}+1} \delta_{m_{y_{c}}, n_{x_{\xi}}} \delta_{m_{z_{c}}, n_{y_{\xi}}}
\end{aligned}
$$

Finally, when $\xi=5$ we have:

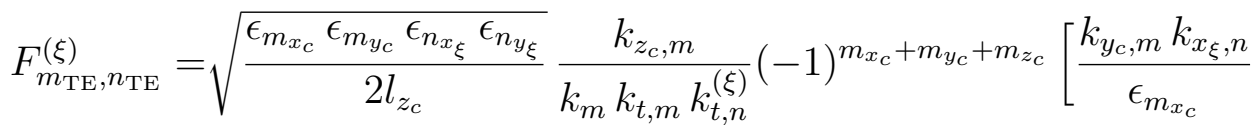

$$
\begin{aligned}
& \left.+\frac{k_{x_{c}, m} k_{y_{\xi}, n}}{\epsilon_{m_{y_{c}}}}\right] \delta_{m_{x_{c}, n_{y_{\xi}}}} \delta_{m_{y_{c}}, n_{x_{\xi}}} \\
& F_{m_{\mathrm{TE}}, n_{\mathrm{TM}}}^{(\xi)}=\sqrt{\frac{\epsilon_{m_{x_{c}}} \epsilon_{m_{y_{c}}}}{2 l_{z_{c}}}} \frac{k_{z_{c}, m}}{k_{m} k_{t, m} k_{t, n}^{(\xi)}}(-1)^{m_{x_{c}}+m_{y_{c}}+m_{z_{c}}}\left[k_{y_{c}, m} k_{y_{\xi}, n}\right. \\
& \left.-k_{x_{c}, m} k_{x_{\xi}, n}\right] \delta_{m_{x_{c}}, n_{y_{\xi}}} \delta_{m_{y_{c}}, n_{x_{\xi}}} \\
& F_{m_{\mathrm{TM}}, n_{\mathrm{TE}}}^{(\xi)}=\sqrt{\frac{\epsilon_{m_{z_{c}}} \epsilon_{n_{x_{\xi}}} \epsilon_{n_{y_{\xi}}}}{l_{z_{c}}}} \frac{1}{2 k_{t, m} k_{t, n}^{(\xi)}}(-1)^{m_{x_{c}}+m_{y_{c}}+m_{z_{c}}+1}\left[k_{x_{c}, m} k_{x_{\xi}, n}\right. \\
& \left.-k_{y_{c}, m} k_{y_{\xi}, n}\right] \delta_{m_{x_{c}}, n_{y_{\xi}}} \delta_{m_{y_{c}}, n_{x_{\xi}}} \\
& F_{m_{\mathrm{TM}}, n_{\mathrm{TM}}}^{(\xi)}=\sqrt{\frac{\epsilon_{m_{z_{c}}}}{l_{z_{c}}}} \frac{1}{k_{t, m} k_{t, n}^{(\xi)}}(-1)^{m_{x_{c}}+m_{y_{c}}+m_{z_{c}}+1}\left[k_{x_{c}, m} k_{y_{\xi}, n}\right. \\
& \left.+k_{y_{c}, m} k_{x_{\xi}, n}\right] \delta_{m_{x_{c}}, n_{y_{\xi}}} \delta_{m_{y_{c}}, n_{x_{\xi}}}
\end{aligned}
$$




\section{Chapter 5}

\section{Analysis of rectangular cavities loaded with a dielectric puck: Resonant frequencies and modal chart}

\subsection{Introduction}

One key aspect related to the use of dielectric resonators is that one may find resonant frequencies of undesired modes (spurious modes) close to the resonant frequency of the operational mode. For this reason, it is crucial to be able to know the resonant frequencies and the field patterns of all the involved modes in order to determine their proper operation and coupling to other elements in the circuit to be designed. The electromagnetic behaviour of the various modes depends on the material properties of the DR (its dielectric permittivity, etc.), its geometric properties (i.e., the diameter/height ratio, etc.), and the surroundings of the DR, this is, the dimensions of the rectangular cavity and the position of the DR inside it. Therefore, if the resonant frequencies and electromagnetic field distributions are accurately computed, it will be possible to determine the most appropriate way to modify the environment of the cavity resonator in order to excite the desired mode, or to avoid as much as possible the excitation of the spurious modes.

The need for an accurate determination of the resonant frequencies and the related electromagnetic field patterns of dielectric resonator modes, together with the mathematical complexity required for solving this kind of structures, have encouraged the emergence of many different approaches for the electromagnetic analysis of this problem [19]. In section 4.2 we have briefly described some techniques that making use of simplified mathematical models, by introducing magnetic walls $[10,11,13,14]$ or by using other approximations [24, 25], allow us to obtain the solution for the $T E_{01 \delta}$ mode of cylindrical dielectric resonators. Van Bladel and others $[26,27]$ made use of the perturbational-asymptotic technique, consisting of a power expansion of the refraction index $\left(n=\sqrt{\varepsilon_{r}}\right)$ in the Maxwell's equations, to analyse high-permittivity isolated cylindrical and ring resonators. Krupka [108] obtained the lowest resonances of a cylindrical anisotropic resonator in a cylindrical cavity by means of the 
Galerkin-Rayleigh-Ritz method.

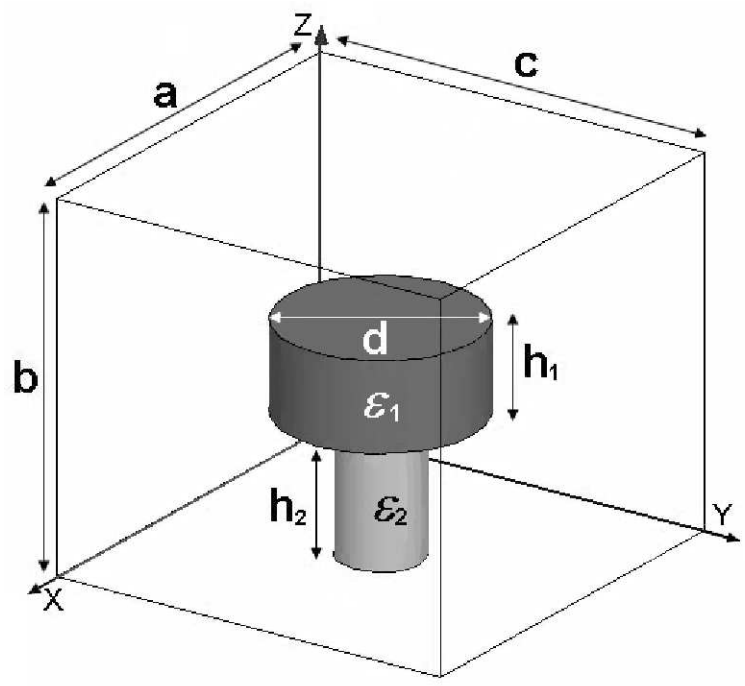

Figure 5.1: Rectangular cavity loaded with a cylindrical dielectric resonator.

In the 1980's more sophisticated methods arose. For instance, the so-called radial ModeMatching (M-M) technique was employed firstly by Kobayashi [21] to analyse a cylindrical DR enclosed in a cylindrical metal cavity. This technique divides the DR cross section into complementary regions where the permittivity is independent of the radial coordinate $(\rho)$. Following this procedure, the field in each region is represented as a superposition of $T M$ and $T E$ functions satisfying the boundary conditions at the perfect electric walls of the cavity. Once the fields are described in each region, the continuity of the tangential electric and magnetic fields is enforced at the boundaries between the different regions. The resulting equations are tested using the appropriate functions, thus obtaining an homogeneous system of equations. The resonant frequencies are obtained as the non trivial solution of such a system, i. e., searching for the frequencies where the determinant of the system matrix vanishes. In a later work, Kobayashi used that approach to determine the optimum DR dimensions for obtaining the best separation of the spurious modes from the desired mode (the $T E_{01 \delta}$ or the $H E_{11 \delta}$ modes were considered) in pillbox and ring resonators [28]. A similar procedure, the so-called axial Mode-Matching technique, was also applied by Zaki And Atia [22], as well as by Zaki and Chen [23], to determine the TE, TM and hybrid modes of cylindrical DRs placed symmetrically in a cylindrical cavity. In this case, the problem is divided into partial regions where the permittivity is independent of the axial coordinate, in such a way that the transverse fields can be expanded in terms of homogeneous or dielectrically loaded cylindrical waveguides at each region. This work was also extended to ring resonators enclosed by cylindrical cavities [29] and to pillbox DRs placed symmetrically in rectangular cavities [30]. This last work was used in subsequent years to design mixed modes dielectric resonators filters with rectangular cavities [31] and dielectric combine filters [32].

Other authors have used the orthogonal expansion method to analyse cylindrical dielec- 
tric resonators in rectangular and cylindrical cavities [33,34], and inhomogeneously dielectric filled cavities [34], as well as structures containing asymmetrically located, partial-height or multilayer inhomogeneous cylindrical DRs [35]. A large variety of investigations about dielectric resonators using the Finite-Element method (FEM) [36-40] can be also found in the literature, as well as Finite Difference approaches in the time-domain (FDTD) [41-44] and in the frequency-domain (FDFD) [45]. Nevertheless, the use of this kind of segmentation methods to deal with these problems typically require high computational resources.

Another kind of methods that have been widely used to analyse dielectric resonators are those based on the integral equation approach solved via the Method of Moments (MoM) [46]. These techniques are based on the solution of an integral equation, rather than a differential one. The integral equation is derived by means of a suitable Green's function, which constitutes the kernel. Once the integral equation is formulated, the Method of Moments is employed to test the equation and transform it into linear algebraic equations that can be solved numerically. We can differentiate basically between two possible integral formulations [47]: the Volume Integral Equation Formulation (VIEF) and the Surface Integral Equation Formulation (SIEF). In the former method, the dielectric resonator is replaced by the equivalent volumetric polarization currents [48]. In the second technique, the surface equivalence principle [49] permits to replace the DR by equivalent electric and magnetic currents on the surface defined by the interface between the dielectric resonator and the homogeneous medium in which it is immersed. In this case, the dielectric body must be homogeneous. The main difficulty of this kind of formulations lies in the ability to find the proper Green's function to solve each problem (as discussed in previous chapters). Many authors have used this kind of approaches to determine the modal chart of DRs in free space or enclosed within metal cavities. Glisson et al. [50,51] used the surface integral equation formulation for bodies of revolution to compute the lowest resonant modes of isolated cylindrical dielectric resonators (including their field pattern). They used the free-space Green's function to obtain the integral equation, and applying the MoM obtained the resonant frequencies by searching the zeros of a matrix determinant. Omar and Schünemann [52] presented a theoretical adaptation of both, the volume and the surface integral equation formulations, to analyse DRs inside homogeneously filled waveguides. They proposed to use the dyadic Green' function for the waveguides instead of the free-space Green's function. Hanson [53] used the VIEF to describe rectangular and cylindrical dielectric resonators in free space and in microwave integrated circuits (MIC's), while Kajfez and others [54] presented a SIEF to analyse axisymmetric cavities loaded with DRs. More recently, F. Arndt [55,56] proposed a new surface integral equation formulation to analyse arbitrarily shaped dielectric resonators inside conventional rectangular cavities and rectangular cavities with rounded corners. F. Arndt utilizes the Rao-Wilton-Glisson basis functions [57] and an adaptive triangular mesh to expand the magnetic and electric currents on the surface of the dielectric resonators. Most of these approaches, however, have a common factor: the use of the free space Green's function. This fact obliges to enforce the boundary conditions on the metallic walls of the shield, thus increasing the number of unknowns of the problem. Nevertheless, this drawback can be avoided by using the dyadic Green's function of the empty cavity that encloses the dielectric resonator. F. Alessandri and others [58] have used the dyadic Green's function of the 
rectangular cavity to analyse DR filters in rectangular waveguides.

The State-Space Integral-Equation (SS-IE) approach developed in the present thesis is a Volume Integral Equation formulated in the Laplace variable domain. As explained in chapter 3, we have used the dyadic Green's function of the empty rectangular cavity formulated in the Coulomb gauge. This choice has allowed us to compute the modal chart of rectangular cavities loaded with cylindrical DR by solving, only once, a matrix eigenvalue problem. Proceeding in this way, we are able to avoid searching the zeros of a matrix determinant as most of the methods presented above do. Furthermore, the choice of a suitable set of basis functions used by the Method of Moments has allowed us to transform analytically the volume integrals involved to surface integrals, thus improving the efficiency of the formulation.

In this chapter, we present the electromagnetic analysis of different rectangular cavities loaded with a dielectric puck using the SS-IE formulation previously derived. The resonant frequencies of low and high order modes are calculated, as well as their electromagnetic field distributions. The influence of geometrical and electric parameters in the computation of the modal chart of the cavity resonator is studied. The results obtained with the new developed formulation are successfully compared with the technical literature as well as with the wellknown commercial tool Ansoft High Frequency Structure Simulator (Ansoft HFSS) [80], which is based on the Finite-Element Method (FEM). The fact that the algorithm used by HFSS is completely different from the SS-IE formulation presented in this work, makes it a good reference to validate the software developed in this thesis. This study will serve us not only to perform a first validation of the method presented, but also to understand the electromagnetic behaviour of the dielectric-loaded rectangular cavities under analysis.

\subsection{Modal chart}

As it has been explained in Chapter 3, in order to calculate the modal chart of a rectangular cavity loaded with a cylindrical dielectric resonator, we have to solve the homogeneous eigenvalue problem described by (3.42). The resonant frequencies of the structure are obtained from the eigenvalues of the solution, whereas the eigenvectors yield to the field distribution of each resonant mode by means of equations (3.43). In this section, we validate the algorithm developed by comparing the results of the new formulation with the technical literature and with the well-known electromagnetic solver Ansoft HFSS. For this purpose, different structures are analysed, and a catalogue of electric and magnetic field patterns for several resonant modes is presented.

As first test case, we compare the results obtained through this thesis formulation with a classical benchmark found in the technical literature [30]. In such a work, K. A. Zaki and X.P. Liang used the axial Mode-Matching technique to analyse cylindrical dielectric resonators enclosed in rectangular cavities. They calculated the resonant frequencies, the field patterns of low-order modes and the coupling levels between adjacent cavities through irises. They compared the resonant frequencies calculated using their method with measurements. In particular, they obtained the resonant frequency of the first hybrid mode $\left(H E_{12}\right)$. For such a 


\begin{tabular}{|c|rrr|}
\hline Case & $\mathbf{1}$ & $\mathbf{2}$ & $\mathbf{3}$ \\
\hline \hline Diameter of the resonator $d(\mathbf{m m})$ & 16.61 & 17.50 & 19.23 \\
Height of the resonator $h_{1}(\mathbf{m m})$ & 5.54 & 5.84 & 6.43 \\
\hline
\end{tabular}

Table 5.1: Dimensions of the test cases extracted from [30] . Rectangular cavity dimensions: $a=25.4 \mathrm{~mm}, b=25.4 \mathrm{~mm}, c=23.37 \mathrm{~mm}$; DR relative permittivity: $\varepsilon_{r_{1}}=38$; DR support height and relative permittivity: $h_{2}=6.99 \mathrm{~mm}, \varepsilon_{r_{2}}=1$.

purpose, three different cases were proposed. The full structure is centered with regard to the $z=0$ plane of the rectangular cavity, whose dimensions are $a=25.4 \mathrm{~mm}, b=25.4 \mathrm{~mm}$, $c=23.37 \mathrm{~mm}$ (see Fig. 6.1). The DR's relative permittivity is $\varepsilon_{r_{1}}=38$. It is supported by a concentric dielectric cylinder with height $h_{2}=6.99 \mathrm{~mm}$, whose relative dielectric permittivity is assumed to be $\varepsilon_{r_{2}}=1$. The diameter and thickness of the dielectric resonator vary for each different case. Table 5.1 shows the dimensions of the DR for the three different cases under consideration.

\begin{tabular}{|c||c|c||c|c||c|c|}
\hline \multicolumn{1}{|c||}{} & \multicolumn{2}{c||}{ Case 1 } & \multicolumn{2}{c||}{ Case 2 } & \multicolumn{2}{c|}{ Case 3 } \\
\hline & $f(\mathrm{GHz})$ & $\operatorname{Err}(\%)$ & $f(\mathrm{GHz})$ & $\operatorname{Err}(\%)$ & $f(\mathrm{GHz})$ & $\operatorname{Err}(\%)$ \\
\hline \hline Meas. [30] & 4.382 & - & 4.153 & - & 3.777 & - \\
SS-IE & 4.406 & 0.55 & 4.179 & 0.63 & 3.791 & 0.37 \\
MM [30] & 4.3880 & 0.14 & 4.1605 & 0.18 & 3.721 & 1.48 \\
FDTD [44] & 4.40 & 0.41 & 4.17 & 0.41 & 3.78 & 0.08 \\
FDFD [45] & 4.383 & 0.02 & 4.144 & 0.21 & 3.749 & 0.74 \\
\hline
\end{tabular}

Table 5.2: Resonant frequencies of the $H E_{12}$ odd mode for the cases presented in Table 5.1. Comparison between measurements [30] and different numerical algorithms: SS-IE formulation developed in the present thesis, the Mode Matching (MM) technique [30], Finite Difference Time-Domain (FDTD) [44] and Finite Difference Frequency-Domain (FDFD) [45] techniques.

During last years, many other authors who have developed different techniques to analyse this kind of structures, have compared their results with those presented by Zaki and Liang in [30]. In Table 5.2, the results obtained with our method are compared with measurements and Mode Matching (MM) [30], Finite Difference Time-Domain (FDTD) [44] and Finite Difference Frequency-Domain (FDFD) [45] techniques. To calculate accurately the resonant frequencies by means of the state-space integral equation (SS-IE) approach, we have used 250 basis functions to expand the polarization vector, and 1000 resonant modes of the empty rectangular cavity in the kernel (to calculate the dyadic Green's function). It can be observed that a good agreement is achieved between the results of the present work and those from the literature.

As mentioned above, the resonant frequencies shown in Table 5.2 correspond to the first hybrid modes existing in the structures under analysis. The $H E$ modes are hybrid electromagnetic modes with respect to the axis of rotation, $\widehat{u}_{z}$ (see Fig. 4.6), of the DR (they have 
non-zero both, $E_{z}$ and $H_{z}$ ). In the technical literature, we can find different ways of denoting this kind of modes. The use of both $H E M, H E$ and $E H$ to denote hybrid modes usually implies that the modes designated $H E$ are considered " $H$ like" and the modes designated $E H$ are considered "E like". This method of designation is analogous to that used in cylindrical dielectric waveguides [109]. It may be quite ambiguous, since it depends on the criteria used to decide one or other notation. For these reason, we will simply denote all the hybrid modes as $H E$ modes.

Different hybrid modes may be distinguished by two or three modal indexes. In the case of open DRs, some authors $[19,50,51]$ use three modal indexes: the first index, $p$, refers to the azimuthal dependence of the mode; the second and third indexes, $q$ and $l$, refer usually to the number of field extrema within the DR radial and axial directions. In the case of DRs symmetrically placed in a cavity, Zaki and Chen [23] proposed only to use two indexes: the first one is related to the angular variation of the mode, whereas the second one classifies the resonant frequencies of the hybrid modes in increasing order without any reference to the modal field distribution. In our case, we will also employ only two indexes in order to simplify the notation. Nevertheless, in this section we will show later on how the hybrid modes order can change dependending on the diameter/thickness ratio $(d / h)$ of the DR. As a consequence, analogously to the open dielectric resonators, we will use the second modal index to refer the number of field extrema within the DR radial direction instead of their frequency order.

\begin{tabular}{|l||c|c|c||c|c|c||c|c|c|}
\hline \multicolumn{1}{|c||}{} & \multicolumn{3}{c||}{ Case 1. $\boldsymbol{f}(\mathbf{G H z})$} & \multicolumn{3}{c||}{ Case 2. $\boldsymbol{f}(\mathbf{G H z})$} & \multicolumn{3}{c|}{ Case 3. $\boldsymbol{f}(\mathbf{G H z})$} \\
\hline & SS-IE & HFSS & Err (\%) & SS-IE & HFSS & Err $(\%)$ & SS-IE & HFSS & Err (\%) \\
\hline$T E_{01}$ & 3.562 & 3.563 & 0.03 & 3.415 & 3.415 & 0.00 & 3.182 & 3.182 & 0.00 \\
$H E_{12}^{e}$ & 4.374 & 4.356 & 0.41 & 4.142 & 4.122 & 0.50 & 3.738 & 3.713 & 0.67 \\
$H E_{12}^{o}$ & 4.406 & 4.393 & 0.29 & 4.179 & 4.164 & 0.36 & 3.791 & 3.774 & 0.45 \\
\hline
\end{tabular}

Table 5.3: Comparison between HFSS and the new SS-IE Formulation: First three resonant modes of the structures presented in Table 5.1.

Moreover, in the case of isolated cylindrical dielectric resonators [51], each of these modes has a degeneracy due to its dependence on the angular coordinate. The hybrid modes can acquire either $\cos (p \varphi)$ or $\sin (p \varphi)$ dependence (even or odd solutions, respectively). In the case of cylindrical DR enclosed in rectangular cavities, this degeneracy do also exist but can be broken by the distances of the dielectric cylinder to the walls of the cavity, this is, by the dimensions $a$ and $c$. Due to the analogy with the isolated cylindrical dielectric resonator, we will distinguish the quasi-degenerated or degenerated modes as even $\left(H E^{e}\right)$ or odd $\left(H E^{\circ}\right)$ modes. Other authors use different notations. For instance, Zaki [30,31] places a Perfect Electric Wall (PEW) or a Perfect Magnetic Wall (PMW) at the symmetry plane at $x=a / 2$ to simplify the problem, denoting the different modes calculated with this procedure by PEW or PMW.

In order to observe the quasi-degeneracy of these hybrid modes, the first three resonant 


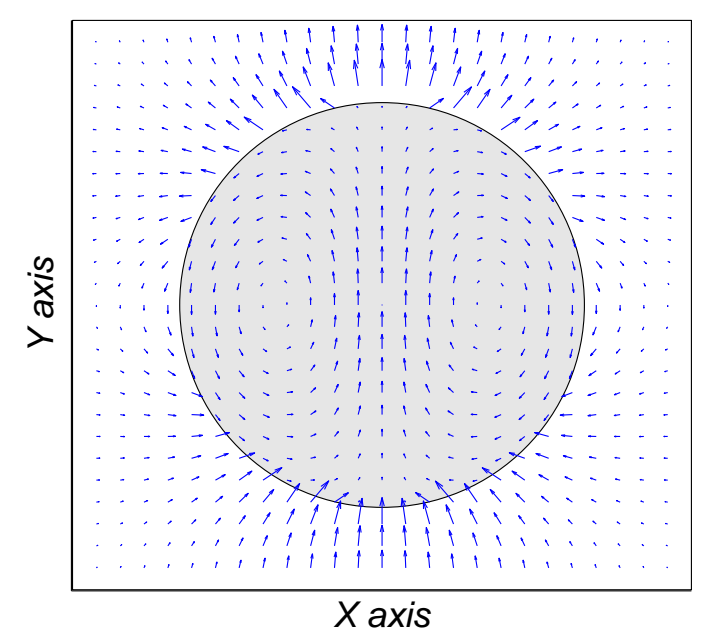

(a) Even mode

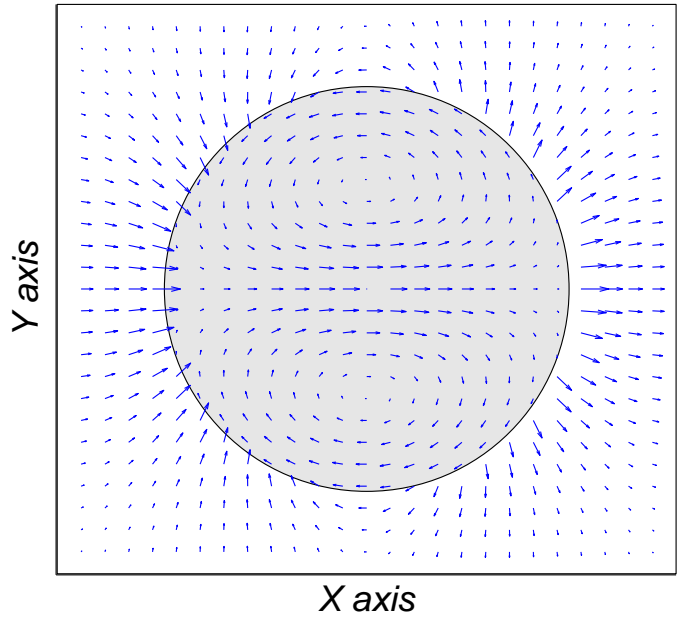

(b) Odd mode

Figure 5.2: Electric field distribution for the first hybrid mode, $H E_{12}$, at the equatorial plane. Rectangular cavity dimensions: $a=25.4 \mathrm{~mm}, b=25.4 \mathrm{~mm}, c=23.37 \mathrm{~mm}$; DR: $d=16.61 \mathrm{~mm}, h_{1}=5.54 \mathrm{~mm}, \varepsilon_{r_{1}}=38$; DR support: $h_{2}=6.99 \mathrm{~mm}, \varepsilon_{r_{2}}=1$.

modes for the test cases presented in Table 5.1 have been calculated with the SS-IE formulation. Table 5.3 gives the comparison between our results and Ansoft HFSS, showing again very good agreement. The second and third modes are the first hybrid modes, $H E_{12}^{e}$ and $H E_{12}^{o}$ respectively. Looking at the EM field distribution, we can observe that the resonant frequencies in Table 5.2 correspond to the third order modes, the odd ones. The separation between the resonant frequencies of both modes is less than a $2 \%$ in all the cases.

Fig. 5.2(a) and Fig. 5.2(b) display the electric field distribution for the $H E_{12}^{o}$ and the $H E_{12}^{e}$ modes, respectively, in the plane X-Y that passes through the center of the dielectric resonator (hereinafter we will call this plane, the equatorial plane). The reference $\varphi=0$ has been chosen to be in the positive- $x$ axis direction (see Fig. 4.6). It can be observed that both modes are orthogonal, and the only factor that breaks the symmetry between both modes is the different separation of the DR to the walls of the cavity in the X or Y direction, i. e., the dimensions $a$ and $c$ of the rectangular cavity. Therefore, the degeneracy of the hybrid modes can be slightly controlled by changing the dimensions of the rectangular cavity. In Fig. 5.3, the resonant frequencies of the $H E_{12}^{o}$ and the $H E_{12}^{e}$ modes are shown as a function of the length of the rectangular cavity (dimension $c$ ). For this purpose, we have used the structure of the test case 1 shown in Table 5.1. As it is expected, the modes are degenerated when $a=c=25.4 \mathrm{~mm}$.

In Fig. 5.4(a), we show the electric field distribution for the $H E_{12}^{(e)}$ mode in the Y-Z plane that passes through the center of the dielectric resonator, which corresponds to the plane with $\varphi=\pi / 2$. Hereinafter we will refer to this plane as the meridian plane with $\varphi=\pi / 2$, whereas the $\mathrm{X}-\mathrm{Z}$ plane that passes through the center of the DR will be denoted as the meridian plane with $\varphi=0$. Fig. 5.4(b) gives the magnetic field pattern for the $H E_{12}^{(o)}$ 


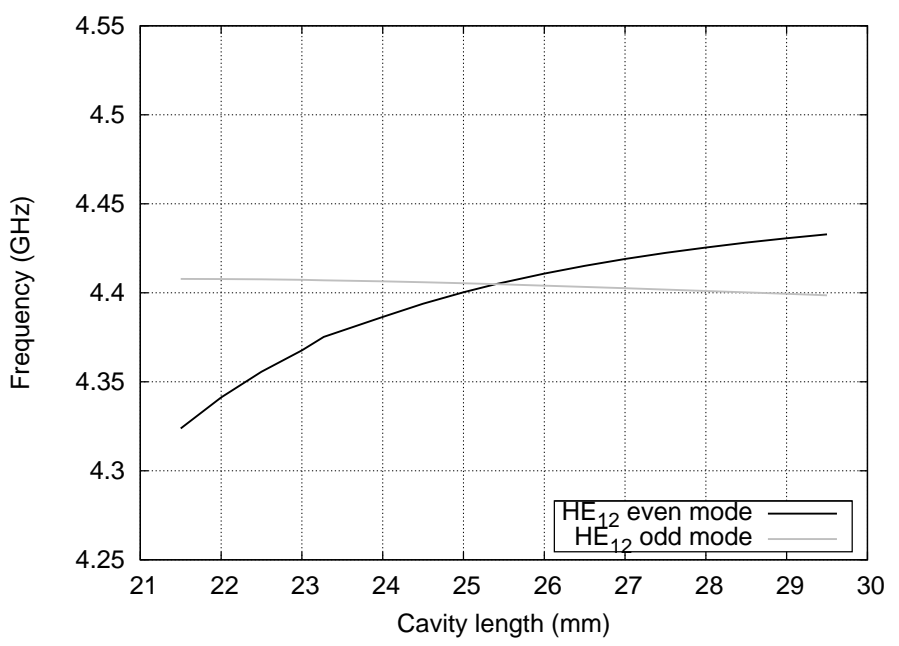

Figure 5.3: Resonant frequencies of the two hybrid modes $H E_{12}$ as a function of the length, dimension $c$, of the rectangular cavity. Rectangular cavity dimensions: $a=25.4 \mathrm{~mm}, b=$ $25.4 \mathrm{~mm}$; DR: $d=16.61 \mathrm{~mm}, h_{1}=5.54 \mathrm{~mm}, \varepsilon_{r_{1}}=38$; DR support: $h_{2}=6.99 \mathrm{~mm}$, $\varepsilon_{r_{2}}=1$.

mode in the meridian plane with $\varphi=\pi / 2$.

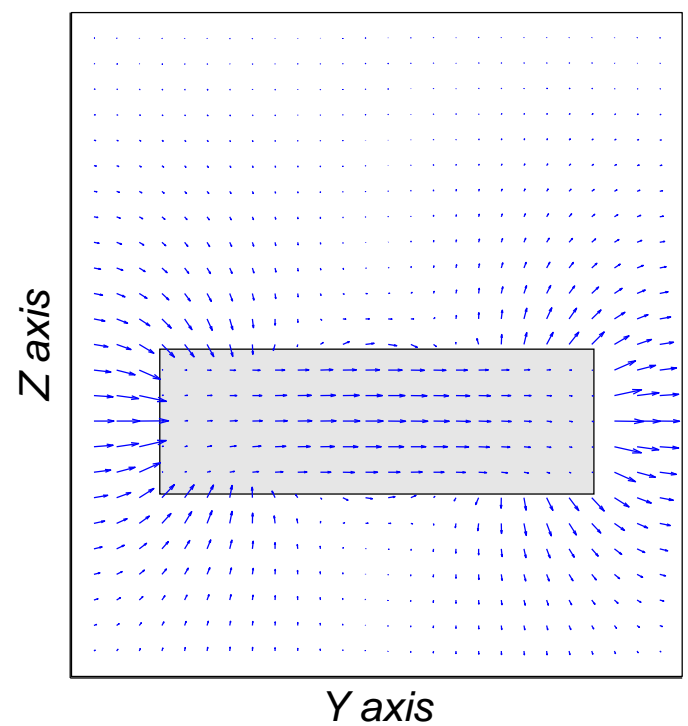

(a) Electric field $\left(H E_{12}^{(e)}\right)$

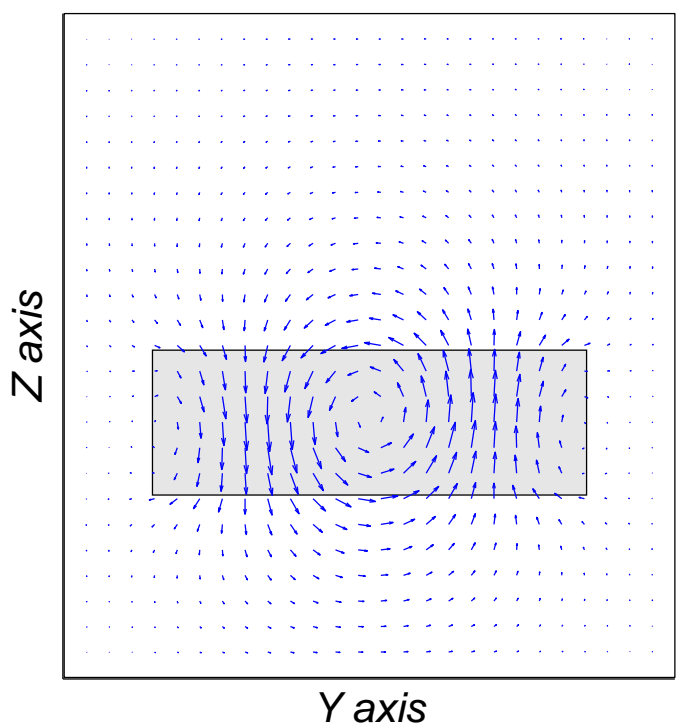

(b) Magnetic field $\left(H E_{12}^{(o)}\right)$

Figure 5.4: Electromagnetic field distribution for the first hybrid mode, $H E_{12}$, in the meridian plane with $\varphi=\pi / 2$. Rectangular cavity dimensions: $a=25.4 \mathrm{~mm}, b=25.4 \mathrm{~mm}$, $c=23.37 \mathrm{~mm}$; DR: $d=16.61 \mathrm{~mm}, h_{1}=5.54 \mathrm{~mm}, \varepsilon_{r_{1}}=38$; DR support: $h_{2}=6.99 \mathrm{~mm}$, $\varepsilon_{r_{2}}=1$. 
So far, a brief analysis of the electromagnetic behaviour of the $H E_{12}$ hybrid modes has been presented. Nevertheless, we can appreciate in Table 5.3 that, for the cases under analysis, these modes are the second and third order ones, respectively. The first order resonant mode is the transverse electric mode, $T E_{01}$, with respect to the axis of rotation of the DR. Fig. 5.5(a) depicts the electric field distribution in the equatorial plane for the $T E_{01}$ mode, whereas Fig. 5.5(b) displays the magnetic field pattern in the meridian plane with $\varphi=\pi / 2$. It can be observed that the electric field is quite strong everywhere within the equatorial plane of the dielectric cylinder, except near the center. Due to this, we could think in removing a cylindrical plug from the center of the dielectric resonator without changing too much the electric field pattern of the mode. Moreover, the presence or absence of dielectric has little impact on the magnetic field, despite of the fact that it is stronger in the center of the DR. Therefore, if we placed a dielectric ring resonator instead a dielectric puck, the resonant frequency of the $T E_{01}$ would not be affected too much, while we could modify the frequencies of other undesired resonant modes in the vicinity of it [19].

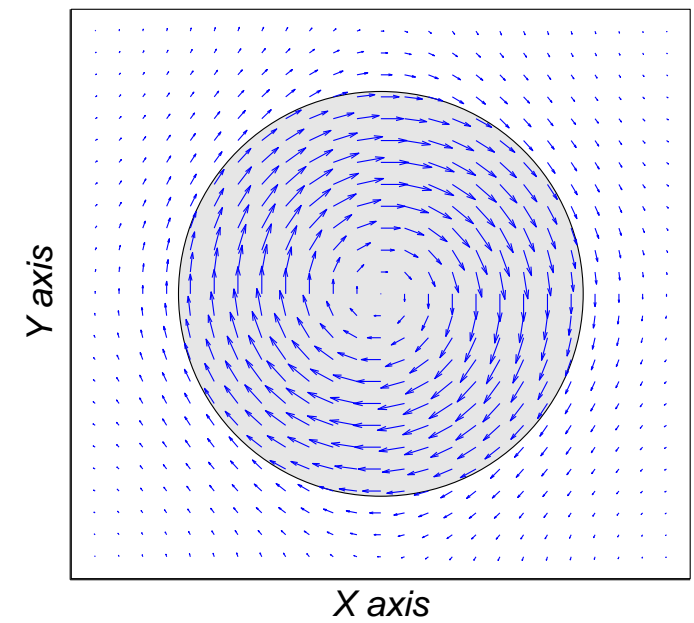

(a) Electric field distribution in the equatorial plane

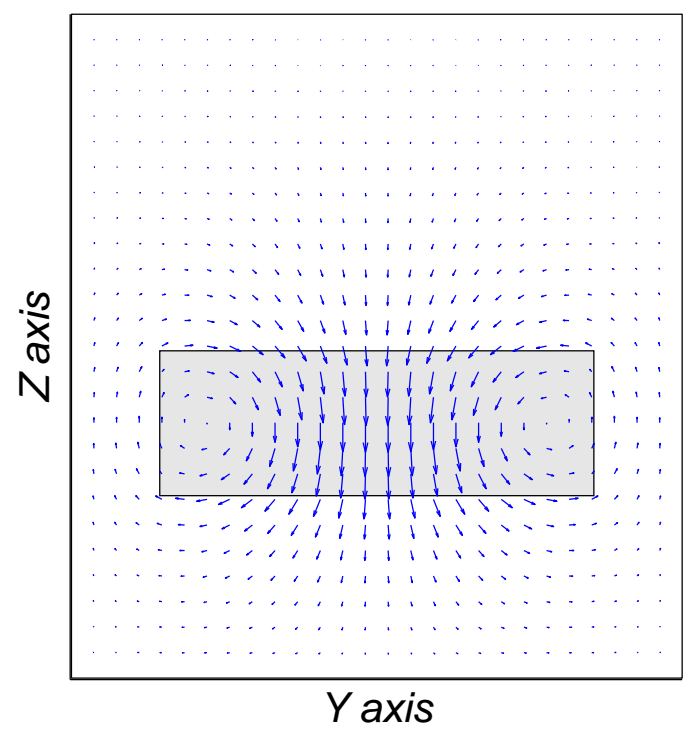

(b) Magnetic field distribution in the meridian plane with $\varphi=\pi / 2$

Figure 5.5: Electromagnetic field distribution for the $T E_{01}$ mode. Rectangular cavity dimensions: $a=25.4 \mathrm{~mm}, b=25.4 \mathrm{~mm}, c=23.37 \mathrm{~mm}$; DR: $d=16.61 \mathrm{~mm}, h_{1}=5.54 \mathrm{~mm}$, $\varepsilon_{r_{1}}=38$; DR support: $h_{2}=6.99 \mathrm{~mm}, \varepsilon_{r_{2}}=1$.

In technological applications [19], the coupling to the $T E_{01}$ mode is often accomplished through the magnetic field via a small horizontal metallic wire loop placed in the equatorial plane. It can also be coupled to a rectangular waveguide operating in the $T E_{10}$ mode via the magnetic field by placing the longest side of the waveguide parallel to the rotation axis of the dielectric cylinder. This coupling procedure is useful in evanescent mode waveguide filters, 


\begin{tabular}{|c|c|c|c|}
\hline Mode order & SS-IE (GHz) & HFSS $(\mathrm{GHz})$ & Relative Error (\%) \\
\hline \hline 1 & 1.899 & 1.899 & 0.00 \\
2 & 2.289 & 2.281 & 0.35 \\
3 & 2.307 & 2.301 & 0.26 \\
4 & 2.688 & 2.687 & 0.04 \\
5 & 2.697 & 2.696 & 0.04 \\
6 & 2.778 & 2.776 & 0.07 \\
7 & 2.869 & 2.866 & 0.10 \\
8 & 2.876 & 2.874 & 0.07 \\
9 & 3.001 & 2.999 & 0.07 \\
10 & 3.013 & 3.011 & 0.07 \\
\hline
\end{tabular}

Table 5.4: Comparison between Ansoft HFSS and the new SS-IE formulation. Resonant frequencies $(\mathrm{GHz})$ of the first 10 modes of a dielectric-loaded resonator cavity: $a=36 \mathrm{~mm}$, $b=30 \mathrm{~mm}, c=40 \mathrm{~mm}$; resonator: $h_{1}=10 \mathrm{~mm}, d=30 \mathrm{~mm}, \varepsilon_{r_{1}}=45$; support: $h_{2}=$ $7.5 \mathrm{~mm}, \varepsilon_{r_{2}}=1$.

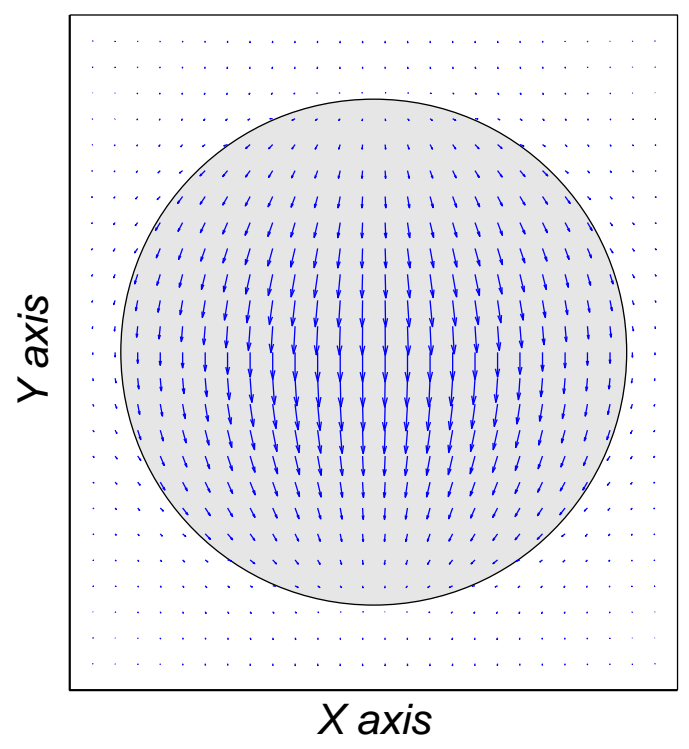

(a) Electric field distribution

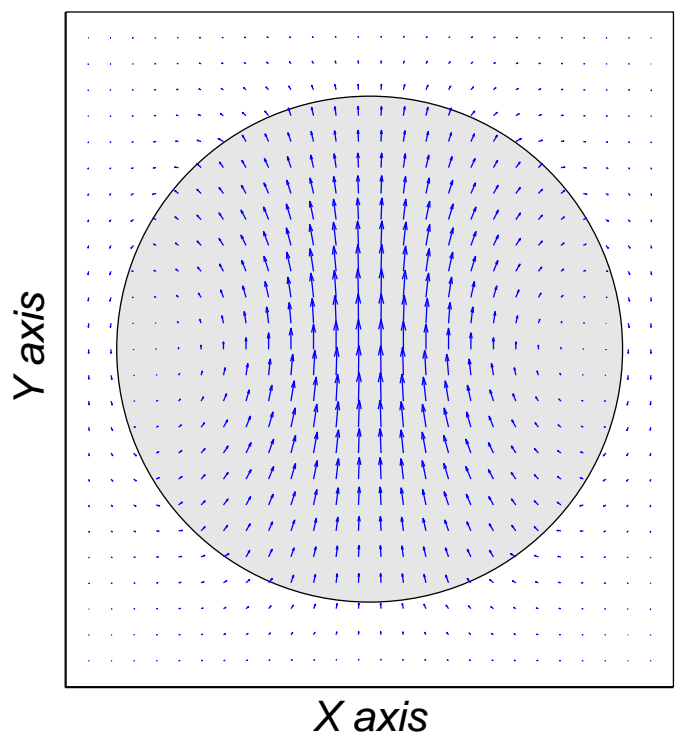

(b) Magnetic field distribution

Figure 5.6: Electromagnetic field distribution in the equatorial plane for the $H E_{11}^{(e)}$ mode. Rectangular cavity dimensions: $a=36 \mathrm{~mm}, b=30 \mathrm{~mm}, c=40 \mathrm{~mm}$; DR: $d=30 \mathrm{~mm}$, $h_{1}=10 \mathrm{~mm}, \varepsilon_{r_{1}}=45$; DR support: $h_{2}=7.5 \mathrm{~mm}, \varepsilon_{r_{2}}=1$.

as we will see in the next chapter. Furthermore, coupling via the electric field can also be performed by using a small horizontal dipole probe placed in the vicinity of the top or the bottom cap of the DR.

The next test case presented consists on a dielectric puck with diameter $d=30 \mathrm{~mm}$, 


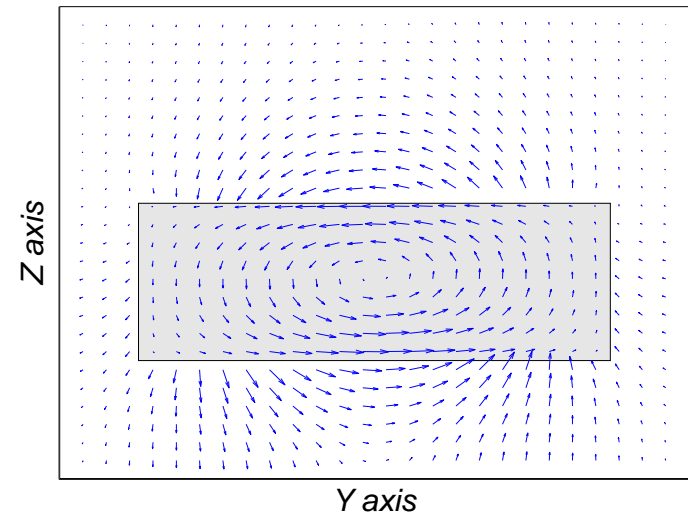

(a) electric field distribution

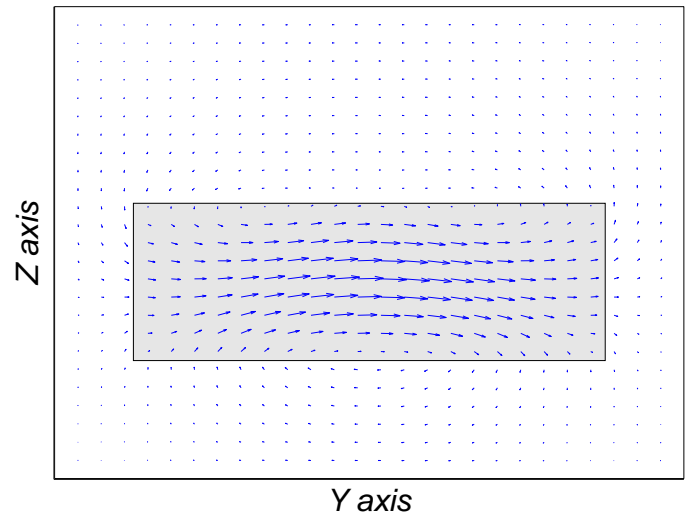

(b) magnetic field distribution

Figure 5.7: Electromagnetic field distribution in the meridian plane with $\varphi=\pi / 2$ for the $H E_{11}^{(e)}$ mode. Rectangular cavity dimensions: $a=36 \mathrm{~mm}, b=30 \mathrm{~mm}, c=40 \mathrm{~mm}$; DR: $d=30 \mathrm{~mm}, h_{1}=10 \mathrm{~mm}, \varepsilon_{r_{1}}=45$; DR support: $h_{2}=7.5 \mathrm{~mm}, \varepsilon_{r_{2}}=1$.

and thickness $h_{1}=10 \mathrm{~mm}$, which is placed inside a rectangular cavity with dimensions $a=36 \mathrm{~mm}, b=30 \mathrm{~mm}$ and $c=40 \mathrm{~mm}$. The relative permittivity of the dielectric resonator is $\varepsilon_{r_{1}}=45$, and it is supported by a concentric dielectric cylinder with height $h_{2}=7.5 \mathrm{~mm}$, and $\varepsilon_{r_{2}}=1$. In Table 5.4, the resonant frequencies of the first 10 modes calculated with the SS-IE formulation are compared with the results provided by Ansoft HFSS, obtaining very good agreement. In order to calculate accurately the resonant frequencies with the SS-IE approach, we have used 250 basis functions and 900 resonant modes of the empty rectangular cavity.

The first three modes of the structure under analysis shown in Table 5.4 are, as in the previous test cases, the $T E_{01}, H E_{12}^{(e)}$ and $H E_{12}^{(o)}$ modes. It can be observed that the $4^{t h}$ and $5^{\text {th }}$ - order resonant modes of the structure, like the $H E_{12}$, are also quasi-degenerated modes. These resonant frequencies correspond to the second hybrid electromagnetic modes, $H E_{11}^{(e)}$ and $H E_{11}^{(o)}$, respectively. The magnetic field distributions for the $H E_{11}^{(e)}$ mode in the equatorial and meridian planes are plotted in Fig. 5.6(b) and 5.7(b), respectively. The electric field of these modes is always zero in the equatorial plane since they present an odd pattern respect to this plane. For this reason, in Fig. 5.6(a) we have displayed the electric field distribution in a plane parallel to the equatorial one, placed $0.2 \mathrm{~mm}$ below the top cap of the DR. Fig. 5.7(a) gives the electric field distribution in the meridian plane. It can be observed that the electric field is strong outside the dielectric resonator top face, where the field is parallel to the DR's cap. Therefore, one could achieve the coupling to the $H E_{11}$ mode by placing a short balanced dipole probe centered on the axis of rotation and oriented parallel to the top surface of the resonator.

In Fig. 5.8 we show the electromagnetic field distribution for the transverse magnetic mode, $T M_{01}$, which corresponds to the $6^{\text {th }}$-order mode of the structure analysed in Table 5.4. Fig. 5.8(a) displays the magnetic field pattern in the equatorial plane, whereas Fig. 5.8(b) 


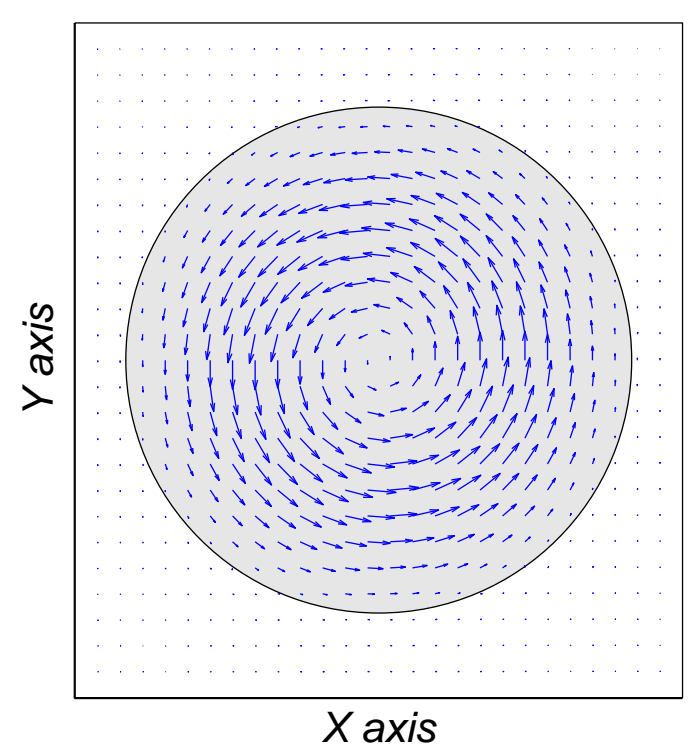

(a) Magnetic field distribution in the equatorial plane

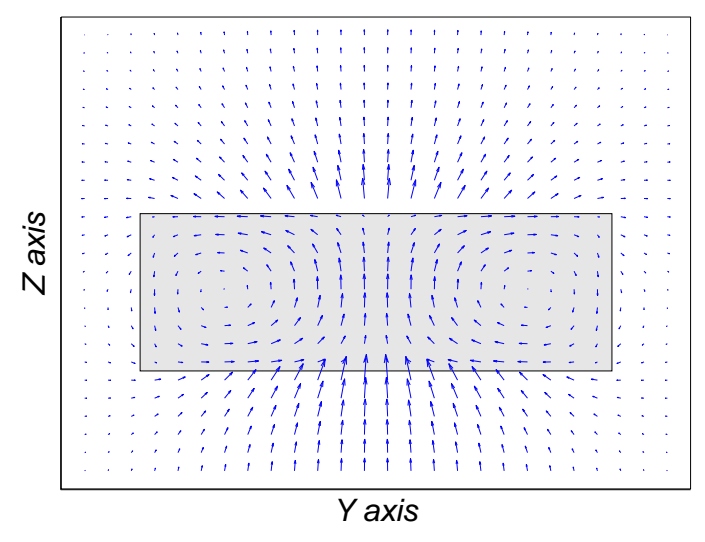

(b) Electric field distribution in the meridian plane with $\varphi=\pi / 2$

Figure 5.8: Electromagnetic field distribution for the $T M_{01}$ mode. Rectangular cavity dimensions: $a=36 \mathrm{~mm}, b=30 \mathrm{~mm}, c=40 \mathrm{~mm}$; DR: $d=30 \mathrm{~mm}, h_{1}=10 \mathrm{~mm}, \varepsilon_{r_{1}}=45$; DR support: $h_{2}=7.5 \mathrm{~mm}, \varepsilon_{r_{2}}=1$.

gives the electric field in the meridian plane with $\varphi=\pi / 2$. It can be observed that this mode is well contained within the dielectric resonator, whereas the electric field is stronger in the center of the top and bottom faces of it. One could say that this mode is like the dual of the $T E_{01}$ mode. The coupling to this mode can be achieved via electric field by a short capacitive probe, a straight coaxial pin, located above or below the DR and parallel to its axis of rotation. It can also be coupled via magnetic field to a rectangular waveguide operating in the $T E_{10}$ mode, for this purpose, the waveguide must be attached to the resonant cavity with its short side parallel to the DR axis of rotation.

The resonant structures that have been analysed so far in this section have a common factor: in all the cases the diameter/thickness ratio of the dielectric resonator is $d / h_{1}=$ 3. Consequently, a rectangular cavity loaded with a cylindrical DR with a different $d / h_{1}$ ratio (e.g., $d / h_{1}=2$ ) is considered as the next test case. The dielectric puck diameter is $d=16.80 \mathrm{~mm}$, and its thickness $h_{1}=6.985 \mathrm{~mm}$. The rectangular cavity dimensions are $a=25.4 \mathrm{~mm}, b=25.4 \mathrm{~mm}$ and $c=23.368 \mathrm{~mm}$. The relative permittivity of the dielectric resonator is $\varepsilon_{r_{1}}=50$, and it is supported by a concentric dielectric cylinder with height $h_{2}=$ $6.985 \mathrm{~mm}$, and relative dielectric permittivity $\varepsilon_{r_{2}}=1$. In Table 5.5, some of the resonant frequencies that correspond to the first 50 modes obtained with the algorithm implement in this work, and those calculated with Ansoft HFSS, are successfully compared. To calculate accurately all the resonant frequencies shown in this table with the SS-IE formulation, we have used 450 basis functions and 1800 resonant modes of the empty rectangular cavity. 


\begin{tabular}{|c|c|c|c|}
\hline Mode order & SS-IE $(\mathrm{GHz})$ & HFSS $(\mathrm{GHz})$ & Relative Error $(\%)$ \\
\hline \hline 1 & 2.779 & 2.779 & 0.00 \\
2 & 3.351 & 3.351 & 0.00 \\
3 & 3.357 & 3.357 & 0.00 \\
4 & 3.529 & 3.520 & 0.26 \\
5 & 3.542 & 3.534 & 0.23 \\
6 & 3.882 & 3.879 & 0.08 \\
7 & 4.062 & 4.062 & 0.00 \\
8 & 4.071 & 4.070 & 0.07 \\
9 & 4.269 & 4.270 & 0.02 \\
10 & 4.533 & 4.530 & 0.07 \\
15 & 4.918 & 4.916 & 0.04 \\
20 & 5.198 & 5.198 & 0.00 \\
25 & 5.776 & 5.755 & 0.36 \\
30 & 5.911 & 5.905 & 0.10 \\
35 & 6.220 & 6.220 & 0.00 \\
40 & 6.421 & 6.408 & 0.20 \\
45 & 6.605 & 6.597 & 0.12 \\
50 & 7.007 & 7.004 & 0.04 \\
\hline
\end{tabular}

Table 5.5: Comparison between Ansoft HFSS and the new SS-IE formulation. Resonant frequencies $(\mathrm{GHz})$ of the first 50 modes of a dielectric-loaded resonator cavity: $a=25.4 \mathrm{~mm}$, $b=25.4 \mathrm{~mm}, c=23.368 \mathrm{~mm}$; resonator: $h_{1}=8.4 \mathrm{~mm}, d=16.8 \mathrm{~mm}, \varepsilon_{r_{1}}=50$; support: $h_{2}=6.985 \mathrm{~mm}, \varepsilon_{r_{2}}=1$.

To solve the Maxwell's equations by means of the FEM technique, HFSS needs to perform an initial 3D mesh of the structure under analysis, which depends on the work frequency. For this reason, we have used different 'setup solutions' to calculate the resonant modes accurately with this software tool. This means that we have split the problem of calculating the first 50 resonant modes, computing groups of ten modes each time. Nevertheless, with our formulation we just need to solve the complete eigenvalue problem only once.

If we display the electromagnetic field distribution in this case, we can observe that the second and third order modes are the hybrid $H E_{11}$ ones, whereas the fourth and fifth correspond to the $H E_{12}$ modes. As a consequence, depending on the $d / h_{1}$ ratio, the order of these hybrid modes can change. For this reason, as we mentioned above, we prefer to use the second modal index to denote the number of extrema field in the radial direction instead of their frequency order.

In Fig. 5.9, the influence of the $d / h_{1}$ ratio in the resonant frequency of the first six modes is studied. For such a purpose, the resonant structure described in Table 5.4 is used. The $d / h_{1}$ ratio is varied keeping constant the volume of the DR. It can be observed that there exists a $d / h_{1}$ interval where the separation between the $T E_{01}$ mode and the contiguous hybrid modes is optimum. For this reason, the ratio typically used to design filters based 
on this kind of resonant structures operating under the $T E_{01}$ mode [18], is comprised within the range $d / h_{1} \in[2-3]$. In Fig. 5.10, the effect of the DR relative permittivity on the resonant frequencies of the first six modes is also presented. To perform this study, we have used a cylindrical dielectric resonator with diameter $d=16.8 \mathrm{~mm}$, and height $h_{1}=8.4 \mathrm{~mm}$ supported by a concentric cylinder of height $h_{2}=6.985 \mathrm{~mm}$, and relative permittivity $\varepsilon_{r_{2}}=$ 1. The rectangular cavity dimensions are $a=25.4 \mathrm{~mm}, b=25.4 \mathrm{~mm}, c=23.37 \mathrm{~mm}$.

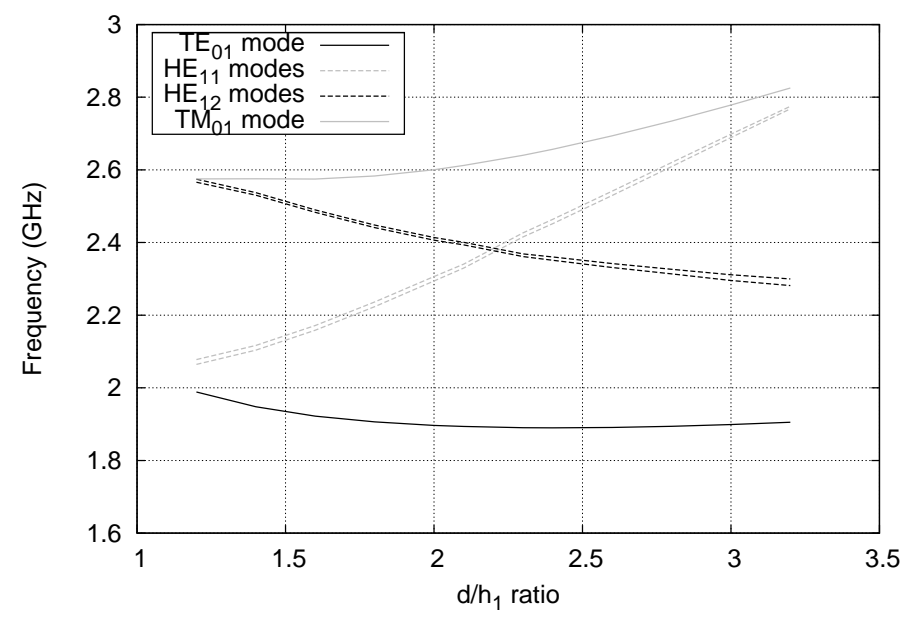

Figure 5.9: Study of the first six resonant modes of a rectangular cavity loaded with a cylindrical dielectric resonator as a function of the $d / h_{1}$ ratio. Rectangular cavity dimensions: $a=36 \mathrm{~mm}, b=30 \mathrm{~mm}, c=40 \mathrm{~mm}$; DR: $\varepsilon_{r_{1}}=45$; DR support: $h_{2}=7.5 \mathrm{~mm}, \varepsilon_{r_{2}}=1$.

\begin{tabular}{c|ccc} 
Mode & SS-IE & Ansoft HFSS & Difference (\%) \\
\hline \hline 1 & 2.768 & 2.769 & 0.04 \\
3 & 3.562 & 3.554 & 0.22 \\
5 & 3.914 & 3.917 & 0.08 \\
10 & 4.480 & 4.481 & 0.02 \\
15 & 5.132 & 5.134 & 0.04 \\
20 & 5.355 & 5.355 & 0.00 \\
25 & 5.853 & 5.841 & 0.20 \\
30 & 6.236 & 6.207 & 0.46 \\
35 & 6.632 & 6.580 & 0.78 \\
40 & 6.869 & 6.872 & 0.04 \\
\hline
\end{tabular}

Table 5.6: Comparison between Ansoft HFSS and the SS-IE formulation. Resonant frequencies of the first 40 modes $(\mathrm{GHz}): a=30 \mathrm{~mm}, b=25.4 \mathrm{~mm}, c=40 \mathrm{~mm}$; resonator: $h_{1}=6.43 \mathrm{~mm}, d_{1}=18 \mathrm{~mm}, \varepsilon_{r_{1}}=50$; support: $h_{2}=11 \mathrm{~mm}, \varepsilon_{r_{2}}=1$. 


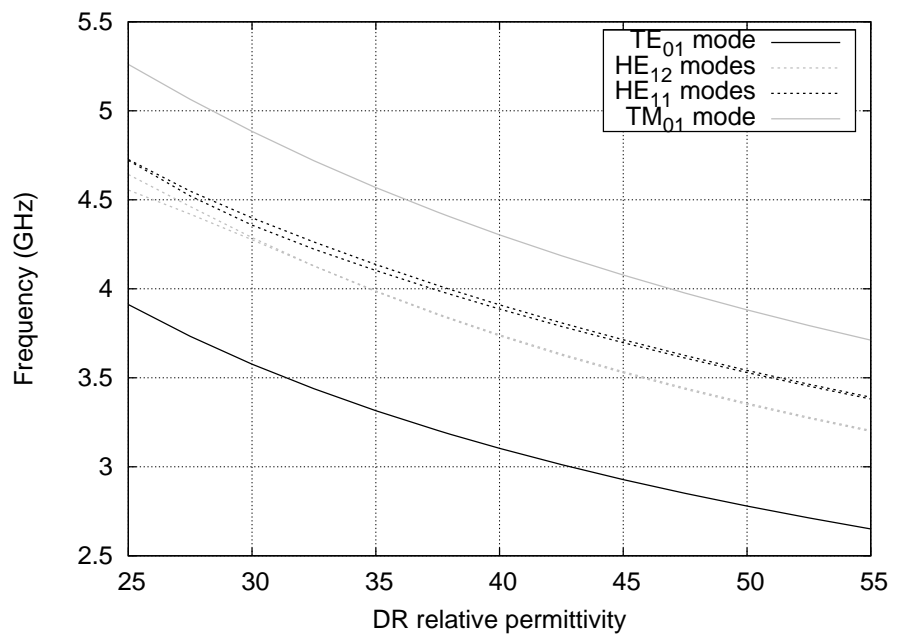

Figure 5.10: Study of the first six resonant modes of a rectangular cavity loaded with a cylindrical dielectric resonator as a function of the DR relative permittivity. Rectangular cavity dimensions: $a=25.4 \mathrm{~mm}, b=25.4 \mathrm{~mm}, c=23.37 \mathrm{~mm}$; DR: $d=16.8 \mathrm{~mm}$, $h_{1}=8.4 \mathrm{~mm}$; DR support: $h_{2}=6.985 \mathrm{~mm}, \varepsilon_{r_{2}}=1$.

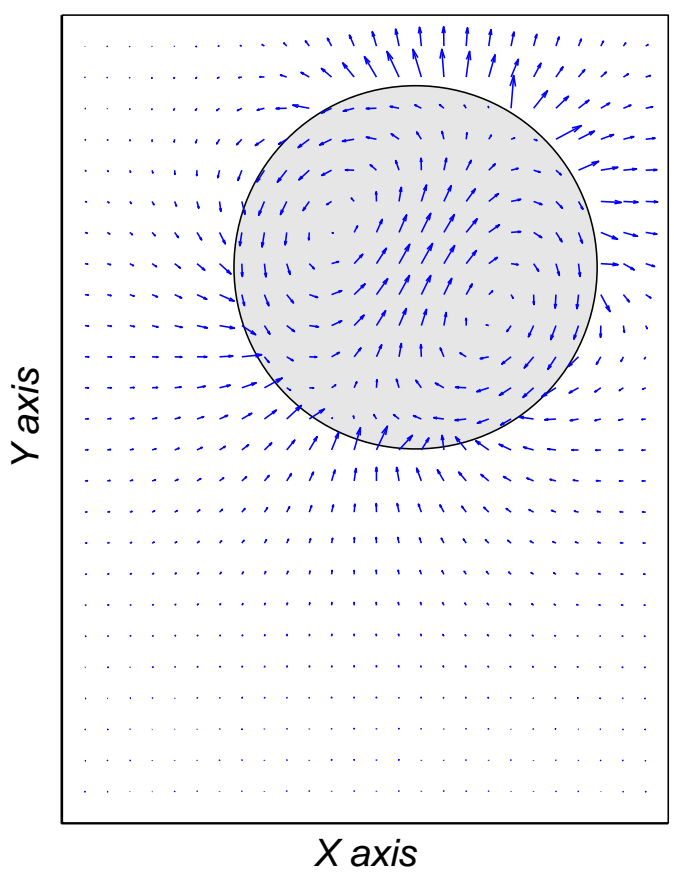

(a) $2^{\text {nd }}$ order mode, $H E_{12}$

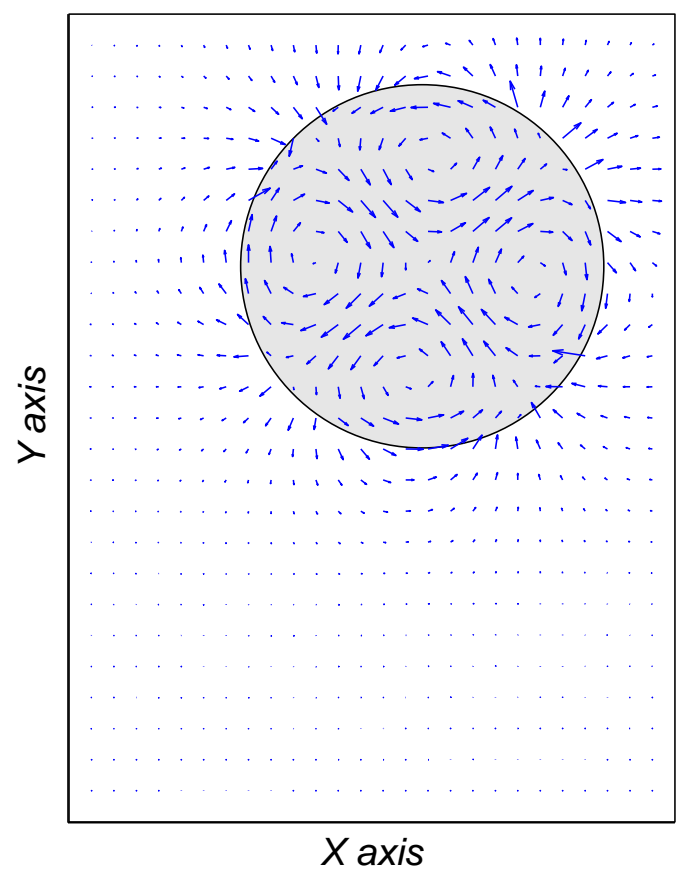

(b) $10^{\text {th }}$ order mode

Figure 5.11: Electric field distribution in the equatorial plane of a rectangular cavity loaded with an off-centred dielectric resonator. Rectangular cavity dimensions: $a=30 \mathrm{~mm}, b=$ $25.4 \mathrm{~mm}, c=40 \mathrm{~mm}$; DR: $h_{1}=6.43 \mathrm{~mm}, d_{1}=18 \mathrm{~mm}, \varepsilon_{r_{1}}=50$; DR support: $h_{2}=$ $11 \mathrm{~mm}, \varepsilon_{r_{2}}=1$, positioned at $a_{o}=17.5 \mathrm{~mm}, c_{o}=27.5 \mathrm{~mm}$. 
On the other hand, when one considers real cases, it is important to have the capability to perform a tolerances analysis. The formulation implemented allows to simulate off-centred structures, which is essential for such practical purpose. For this reason, the last validation test proposed in this section is based on a rectangular cavity with dimensions $a=30 \mathrm{~mm}$, $b=25.4 \mathrm{~mm}, c=40 \mathrm{~mm}$. The cavity is loaded with a dielectric puck with relative permittivity $\varepsilon_{r_{1}}=50$, whose height and diameter are $h_{1}=6.43 \mathrm{~mm}, d_{1}=18 \mathrm{~mm}$, respectively. The center of the base of the DR is positioned at coordinates $a_{o}=17.5 \mathrm{~mm}, c_{o}=27.5 \mathrm{~mm}$ in the $z=0$ plane (see Fig. 4.6). The support height is $h_{2}=11 \mathrm{~mm}$, whereas its relative permittivity is $\varepsilon_{r_{2}}=1$. The first 40 modes have been calculated and compared (see Table 5.6) with Ansoft HFSS, obtaining again a very good agreement. To compute such 40 modes with a high degree of accuracy, 200 basis functions and 1800 resonant modes of the empty rectangular cavity were used. Electromagnetic distributions patterns have been plotted for several modes of this off-centred structure, which can be seen in Figs. 5.11, 5.12 and 5.13. It can be observed that the behaviour of the electromagnetic pattern for the different resonant modes is analogous to the centered case.

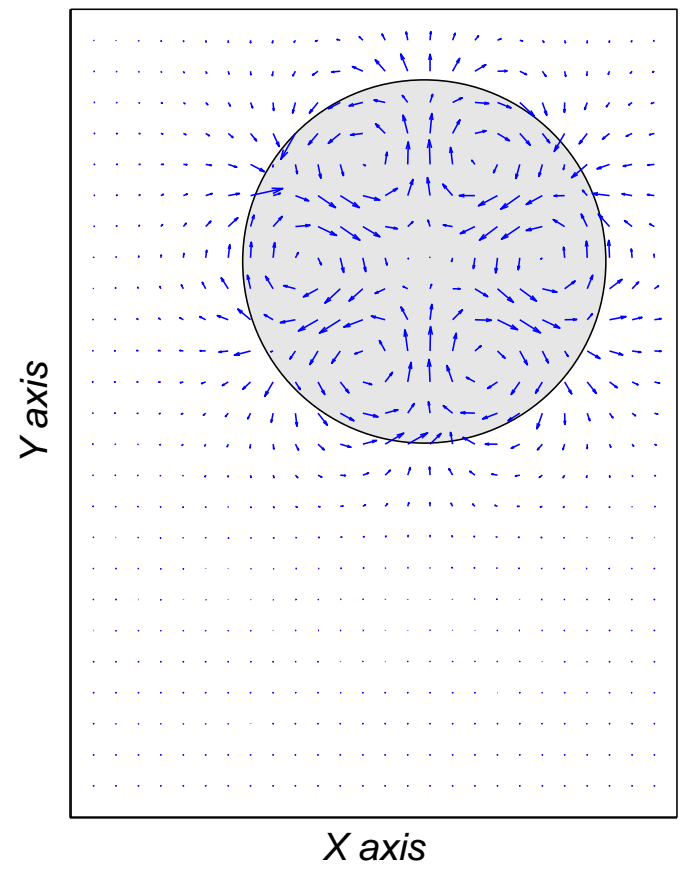

(a) Electric field of the $20^{\text {th }}$ order mode

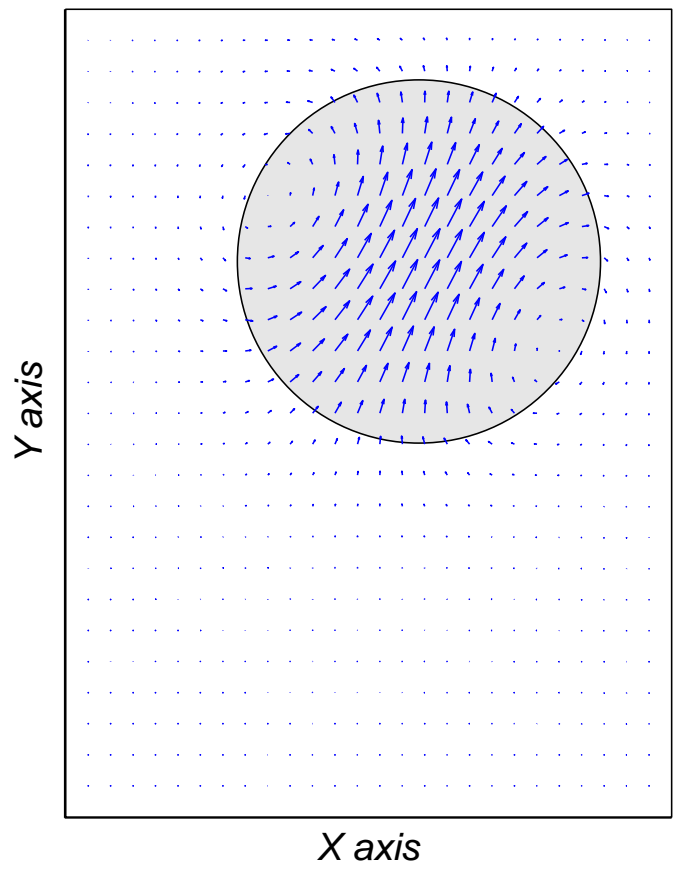

(b) Magnetic field of the $5^{\text {th }}$ order mode

Figure 5.12: Electromagnetic field distribution in the equatorial plane of a rectangular cavity loaded with an off-centred dielectric resonator. Rectangular cavity dimensions: $a=30 \mathrm{~mm}$, $b=25.4 \mathrm{~mm}, c=40 \mathrm{~mm}$; DR: $h_{1}=6.43 \mathrm{~mm}, d_{1}=18 \mathrm{~mm}, \varepsilon_{r_{1}}=50$; DR support: $h_{2}=11 \mathrm{~mm}, \varepsilon_{r_{2}}=1$, positioned at $a_{o}=17.5 \mathrm{~mm}, c_{o}=27.5 \mathrm{~mm}$.

Finally, we present a detailed convergence analysis for a better understanding of the 


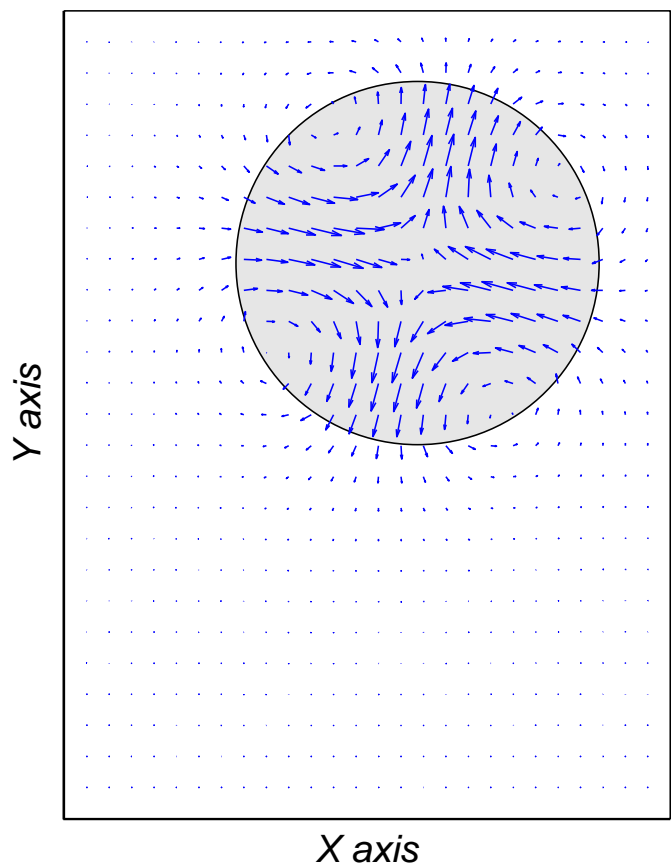

(a) Magnetic field of the $10^{\text {th }}$ order mode

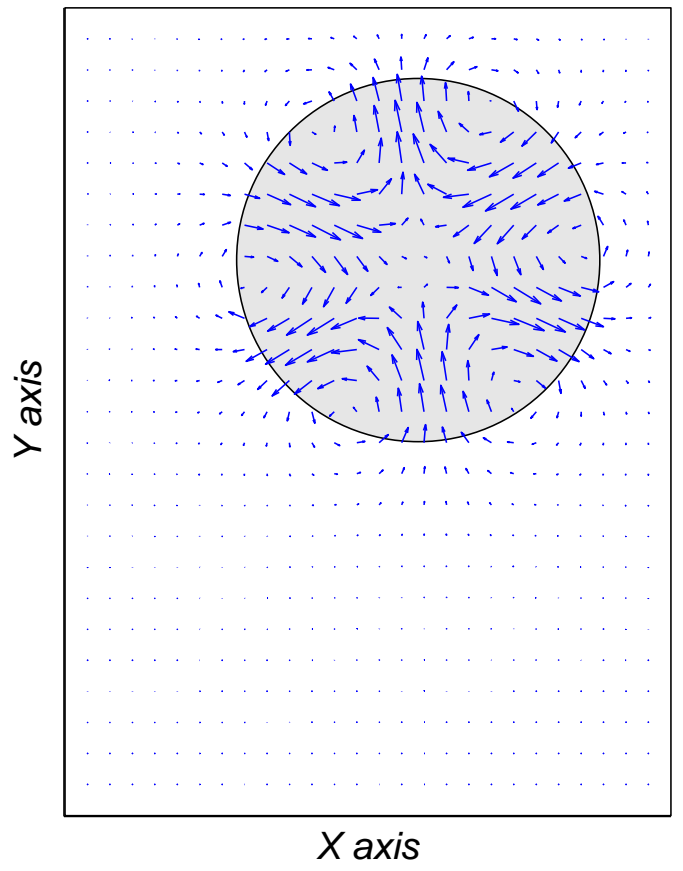

(b) Magnetic field of the $15^{\text {th }}$ order mode

Figure 5.13: Magnetic field distribution in the equatorial plane of a rectangular cavity loaded with an off-centred dielectric resonator. Rectangular cavity dimensions: $a=30 \mathrm{~mm}, b=$ $25.4 \mathrm{~mm}, c=40 \mathrm{~mm}$; DR: $h_{1}=6.43 \mathrm{~mm}, d_{1}=18 \mathrm{~mm}, \varepsilon_{r_{1}}=50$; DR support: $h_{2}=$ $11 \mathrm{~mm}, \varepsilon_{r_{2}}=1$, positioned at $a_{o}=17.5 \mathrm{~mm}, c_{o}=27.5 \mathrm{~mm}$.

algorithm developed. The relevant parameters to perform such a study are:

- $Q$, which represents the number of basis functions employed to expand the equivalent polarization vector following the MoM (see eq. (3.32)).

- $M$, which is the number of modes of the empty rectangular cavity used in the resonant mode expansion (RME) of the dyadic Green's functions for the electric and magnetic vector potentials (see eq. (2.84) and (3.27)).

As it has been described in sections 2.6 and 3.3, we use a resonant mode expansion to compute the dyadic Green's functions for the vector potentials in the Coulomb's gauge. The series involved in such expressions are truncated by retaining the first $\mathrm{M}$ resonant modes of the empty rectangular cavity, $\vec{E}_{m}$, that satisfy:

$$
k_{m} \leq k_{M} \leq \xi k_{\max } \leq k_{M+1},
$$

where $k_{\max }$ is the wavenumber correspondent to the highest frequency in the band of interest, and $\xi$ is an accuracy factor. These expressions for the dyadic Green's function have been widely used in other works $[62,63,73,74]$, where the value of the accuracy factor has been typically chosen around $\xi \in[2,3]$. Nevertheless, in our case the value of $k_{\max }$ is given by 
the resonant frequencies of the dielectric-loaded cavity resonator. And, due to the presence of the dielectric resonator, these resonant frequencies are much lower than the frequencies of the resonant modes of the empty rectangular cavity. This means that following the cited criteria, even taking $\xi \in[3,4]$, in most of the cases we will retain in the Green's function expansion very few resonant modes of the empty cavity, thus obtaining inaccurate results. As an example, we can observe the test case 3 in Table 5.1. If we analyse an structure operating with the fundamental mode in the frequency range $2-4 \mathrm{GHz}$, we will have a ratio $k_{\text {max }} / k_{1}=2.1$, being $k_{1}$ the first order resonant mode of the empty rectangular cavity. For instance, taking $\xi=4$, we will only need to retain 15 resonant modes of the rectangular cavity in the RME of the dyadic Green's function.

Moreover, the difference between the value of $k_{\max }$ and the wavenumber associated to the highest order rectangular cavity mode $\left(k_{M}\right)$, used in the resonant mode expansion, will critically depend on the dimensions of the DR as well as on its relative permittivity. Thus, the appropriate value of the accuracy factor will present strong variations, depending on the structure under analysis.

For this reason, in order to obtain accurate results, we have followed an additional criteria to select the number of rectangular cavity modes to be used in the resonant mode expansion. As it is well known, each resonant mode $\left(\vec{H}_{m}, \vec{E}_{m}\right)$ used in this expansion has associated a resonant wavenumber $k_{m}$. Thus, the wavenumber of the highest order resonant mode employed, $k_{M}$, can give us an idea of the maximum spatial resolution that is used to describe the dyadic Green's functions involved in the integral equation. The bigger is $k_{M}$, the highest spatial resolution is used. On the other hand, the polarization vector is also expanded in terms of the basis functions $\{\vec{w}\}$. Each basis function, $\vec{w}_{q}$, has also a related wavenumber, $K_{q}$, associated. Thus, the spatial resolution employed to expand the unknown of the problem, the polarization vector $(\vec{P})$, is somehow controlled by the value of the wavenumber of the highest order basis function, $K_{Q}$. It has no sense to compute the dyadic Green's function with high spatial resolution if the polarization vector is computed with low resolution and vice versa. Therefore, the additional criteria that we have used is to retain the rectangular cavity modes that describe the dyadic Green's functions with a similar spatial resolution to that used for expanding the polarization vector with the basis functions. This is:

$$
k_{m} \leq k_{M}, \quad \text { and } \quad K_{q} \leq K_{Q}, \quad \text { with } k_{M} \sim K_{Q}
$$

Following this last criteria, we directly relate the number of resonant modes (M) used in the RME to the number of basis functions employed to expand $\vec{P}$. Therefore, the most relevant parameter to take into account in the convergence study is the number of basis functions (Q) used by the MoM.

In Figs. 5.14, 5.15, 5.16 and 5.17, the convergence study that we have performed is shown. These figures display the resonant frequencies of the first order modes for different dielectric-loaded resonator cavities as a function of $Q$. Among all the geometrical and electrical parameters that describe a rectangular cavity loaded with a cylindrical resonator, those who have a biggest influence on the resonant modes of the structure are the dimensions and the relative permittivity of the dielectric resonator. For this reason, we have focused our 
attention in analysing the behaviour of structures with different relative permittivities $\varepsilon_{r_{1}}$ and different $d / h_{1}$ ratios of the dielectric resonator. Moreover, in all the considered cases the dielectric resonator is centered with respect to the base of the rectangular cavity, so $a_{o}=a / 2$ and $b_{o}=b / 2$.

In these graphics, we have plotted the evolution of each resonant frequency in terms of the number of basis functions with two different lines, in order to distinguish between the two criteria used to sort the basis functions $\vec{w}_{q}$ :

- A first one that consists in sorting the basis functions from the lower to higher related wavenumbers. This is what we have called the ' $k$-order criteria'.

- A second criteria that is based on the modal indexes $(p, q, l)$ associated with each basis function (see Table 4.1). We have created a new index:

$$
\Omega=p+q+l
$$

In this way, we can sort the basis functions from lower to higher $\Omega$ index. Those basis functions that have the same $\Omega$ index, are sorted according to their wavenumber. This is what we have called the 'indexes-order criteria'.

In Fig. 5.14, the evolution of the resonant frequency of the $T E_{01}$ and the hybrid $H E_{12}$ modes are shown (see Figs. 5.14(a) and 5.14(b), respectively) for a rectangular cavity loaded with a DR with a ratio $d / h_{1}=3$ and a relative permittivity $\varepsilon_{r_{1}}=38$. To perform this analysis we have used the structure of the case 3 in Table 5.1. We can observe how the $T E_{01}$ mode reach convergence much more faster than the hybrid modes. We only need 100 basis functions to have a convergent result for the $T E_{01}$ mode, even it is enough with 50 basis function making use of the 'indexes-order criteria'. On the other hand, to reach convergence for the $H E_{12}$ modes, around 400 basis functions are needed with the 'k-order criteria', and around 200 if we use the 'indexes-order criteria'. If we depict the same graphic for the $T M_{01}$ and other hybrid modes, such as the $H E_{11}$ ones, we will observe a similar pattern. In fact, this behaviour is repeated in all the cases under consideration for the convergence study. The reason for this is that we have expanded the polarization vector, which is proportional to the electric field inside the DR, in terms of $T E^{z}$ and $T M^{z}$ basis functions. Thus, the electric field corresponding to $T E$ and $T M$ dielectric modes match more better with this kind of basis functions than with the hybrid ones.

The same resonant modes are analysed in Fig. 5.15 for a structure with a DR ratio $d / h_{1}=3$ and a relative permittivity $\varepsilon_{r_{1}}=50$. In particular, the test case 1 in Table 5.1 has been used by changing the relative permittivity of the dielectric resonator. As it is observed, the convergence pattern is analogous to the one presented in Fig. 5.14. Nevertheless, this behaviour changes when we analyse a rectangular cavity loaded with a dielectric resonator with a ratio $d / h_{1}=2$ as it is shown in Fig. 5.16. Figs. 5.16(a) and 5.16(b) display the resonant frequency of the $T E_{01}$ and the $H E_{12}$ modes, respectively, as a function of Q. The rectangular cavity dimensions are $a=25.4 \mathrm{~mm}, b=25.4 \mathrm{~mm}, c=23.368 \mathrm{~mm}$; the 
dielectric resonator dimensions are $h_{1}=8.4 \mathrm{~mm}$ and $d_{1}=16.8 \mathrm{~mm}$, whereas its relative permittivity is $\varepsilon_{r_{1}}=38$; the support's height is $h_{2}=6.99 \mathrm{~mm}$.

By comparing Fig. 5.14 and Fig. 5.16, we can observe that the $T E_{01}$ mode reach convergence at the same level, but the hybrid modes reach convergence much faster for the $d / h_{1}=2$ case than for the $d / h_{1}=3$ one. To explain this different behaviour, one should first remember that each basis function has associated the modal indexes $p, q$ and $l$, which are related to the angular, radial, and the axial coordinates $(\varphi, \rho, z)$ of the cylindrical coordinate system where the basis functions are defined (see Table 4.1). When a wide dielectric resonator (e. g. $d / h_{1}=3$ ) is analysed, and the basis functions are sorted by their wavenumbers, the different values that the modal indexes can take are not balanced. For instance, in the case studied in Fig. 5.14, if we take the first 200 basis functions sorted by their wavenumber, the maximum values that the modal indexes reach are $(p, q, l)=(8,3,2)$, and most of them with $l=0,1$. Thus, in this case the basis functions selected are suitable to describe the variation of the fields in the angular and radial coordinates, whereas the variation in the axial coordinate will be weakly characterized. Nevertheless, when the radius of the dielectric resonator approaches to its height $\left(d / h_{1}=2\right)$, the modal indexes are more balanced, thus contributing to a faster convergence. This fact has a bigger influence in the convergence of the hybrid modes, since the electric field of these modes is matched in a worse way through the basis functions used. Indeed, due to this reason, we have proposed a new criteria to sort the basis functions. As explained before, we calculate the $\Omega$ index which is the sum of the 3 modal indexes related to each basis function. Thus, sorting the basis functions from lower to higher $\Omega$ indexes, we manage to ensure that the set of basis functions selected has enough variation of all the three modal indexes $(p, q, l)$. In each graphic presented in the convergence analysis, we have plotted the convergence of the algorithm using the criteria based on the classification of the basis functions by their wavenumber ('k-order criteria') and using this new criteria ('indexes-order criteria'). It can be observed that the convergence is enhanced by means of the 'indexes-order criteria' in all the cases.

Finally, we have plotted the evolution of the resonant frequencies for the $T M_{01}$ and $H E_{11}$ modes of a structure with a dielectric resonator whose relative permittivity is $\varepsilon_{r_{1}}=50$ and its ratio is $d / h_{1}=2$, observing the previous tendency. 


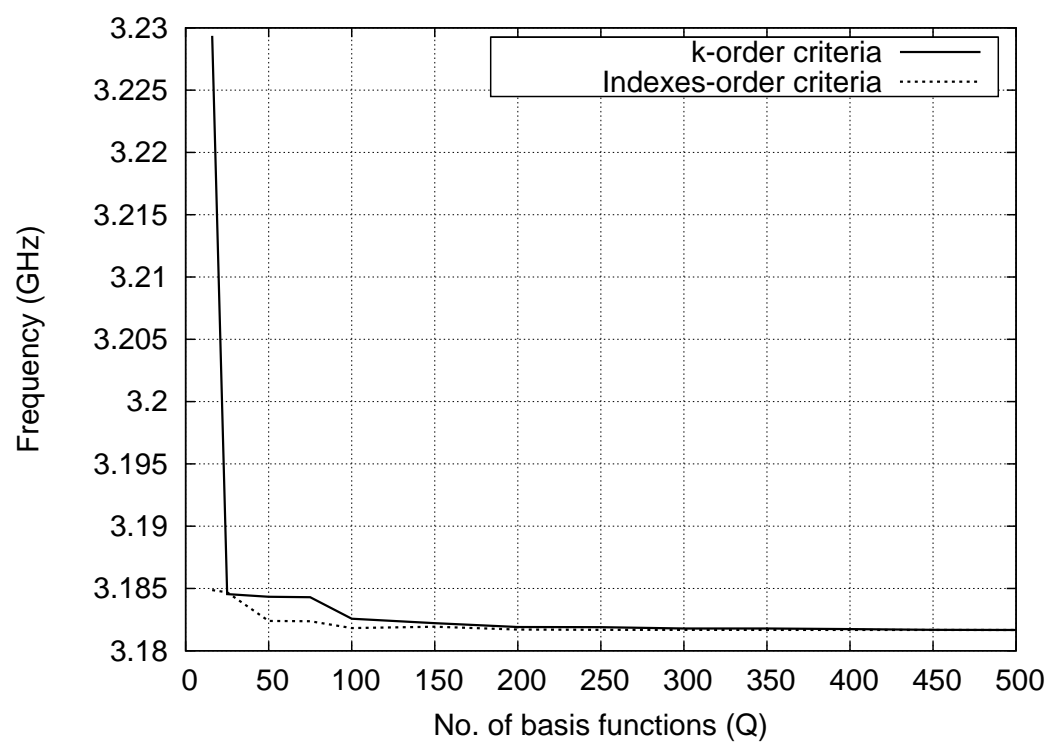

(a) $T E_{01}$ mode

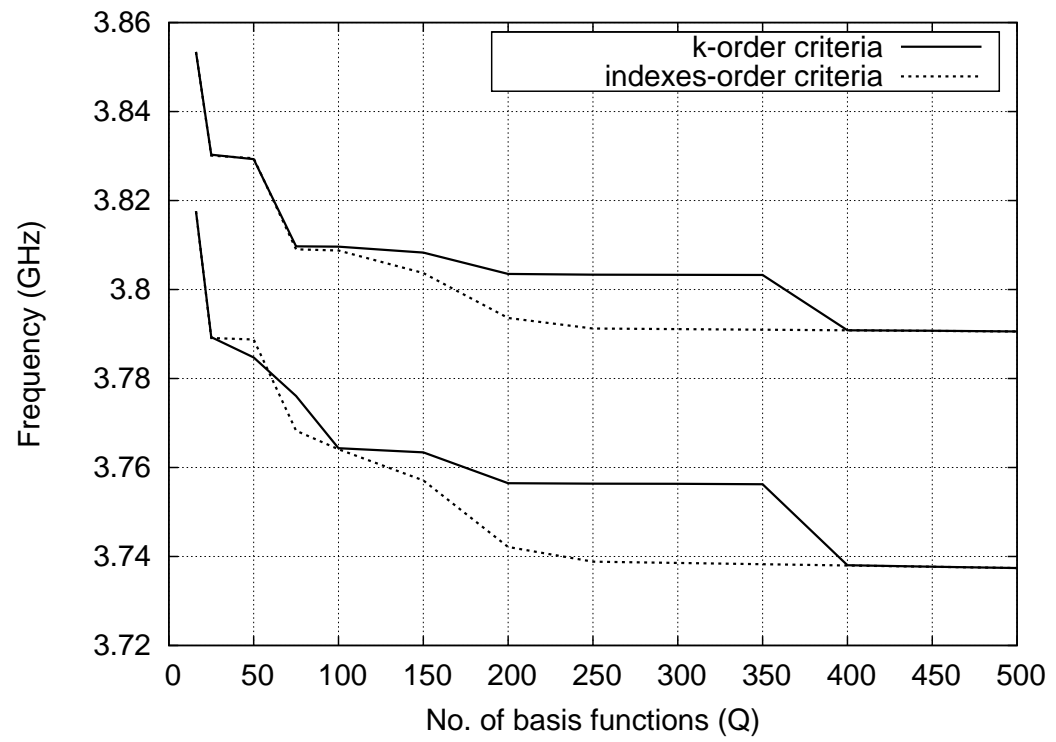

(b) $H E_{12}$ modes

Figure 5.14: Convergence study: Resonant frequencies as a function of the number of basis functions (Q) used by the MoM. Rectangular cavity dimensions: $a=25.4 \mathrm{~mm}$, $b=25.4 \mathrm{~mm}, c=23.368 \mathrm{~mm}$; DR: $h_{1}=6.43 \mathrm{~mm}, d=19.23 \mathrm{~mm}, \varepsilon_{r_{1}}=38$; DR support: $h_{2}=6.99 \mathrm{~mm}, \varepsilon_{r_{2}}=1$. 


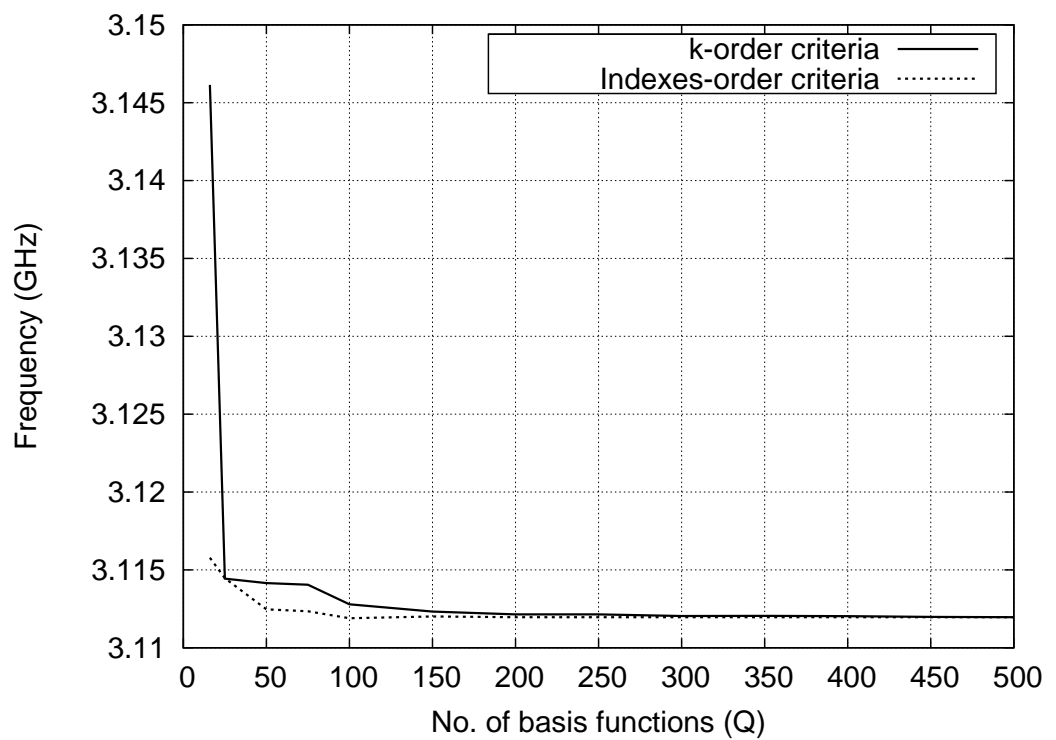

(a) $T E_{01}$ mode

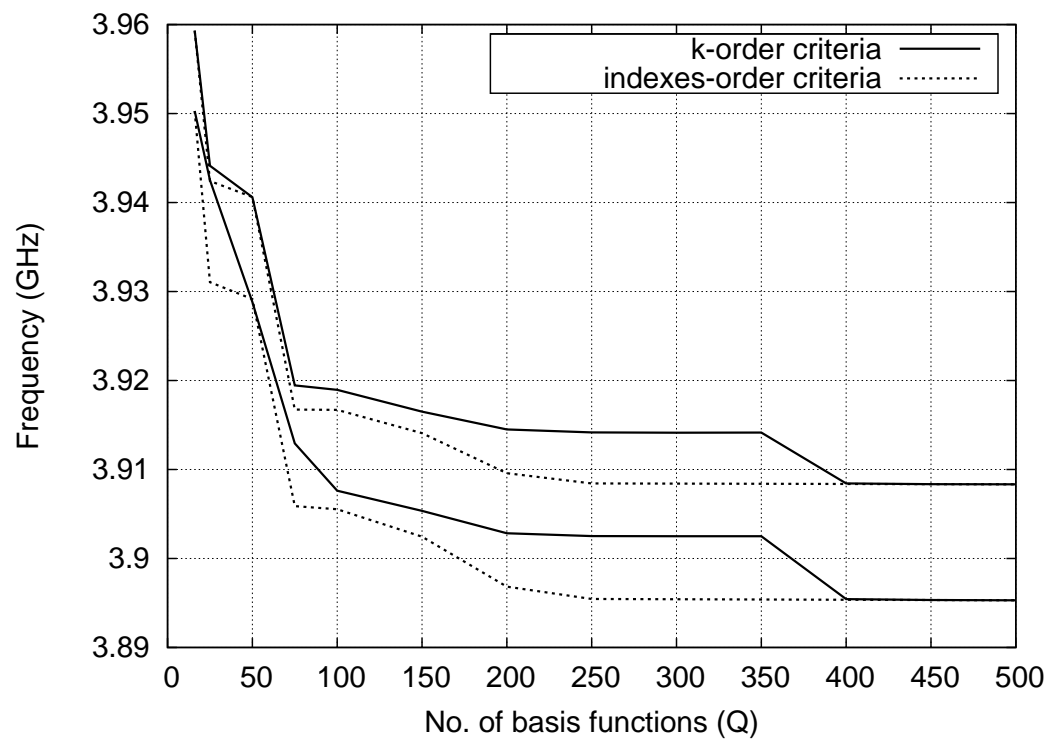

(b) $H E_{12}$ modes

Figure 5.15: Convergence study: Resonant frequencies as a function of the number of basis functions (Q) used by the MoM. Rectangular cavity dimensions: $a=25.4 \mathrm{~mm}$, $b=25.4 \mathrm{~mm}, c=23.368 \mathrm{~mm}$; DR: $h_{1}=5.54 \mathrm{~mm}, d=16.61 \mathrm{~mm}, \varepsilon_{r_{1}}=50$; DR support: $h_{2}=6.99 \mathrm{~mm}, \varepsilon_{r_{2}}=1$. 


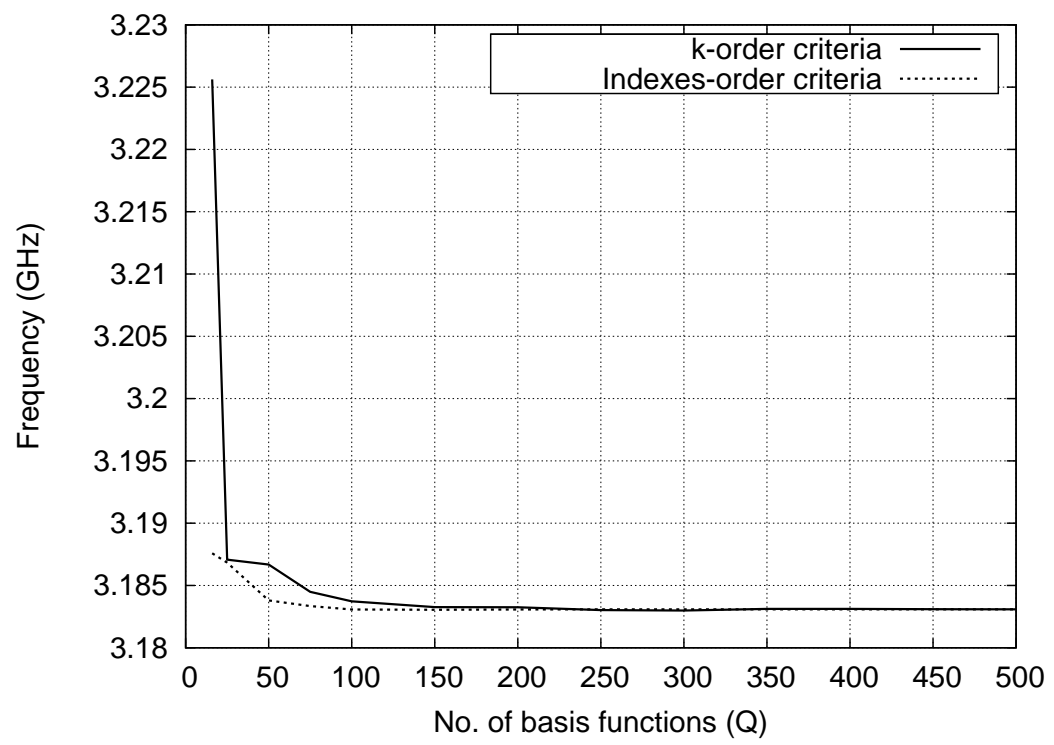

(a) $T E_{01}$ mode

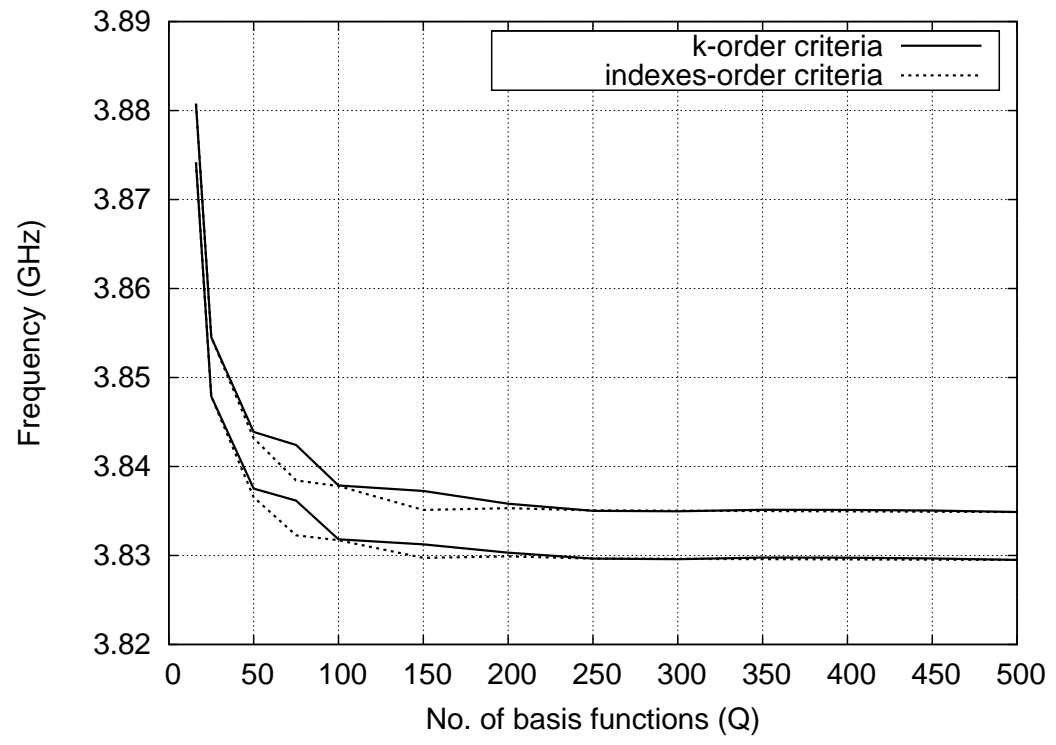

(b) $H E_{12}$ modes

Figure 5.16: Convergence study: Resonant frequencies as a function of the number of basis functions (Q) used by the MoM. Rectangular cavity dimensions: $a=25.4 \mathrm{~mm}$, $b=25.4 \mathrm{~mm}, c=23.368 \mathrm{~mm}$; DR: $h_{1}=8.4 \mathrm{~mm}, d=16.8 \mathrm{~mm}, \varepsilon_{r_{1}}=38$; DR support: $h_{2}=6.99 \mathrm{~mm}, \varepsilon_{r_{2}}=1$. 


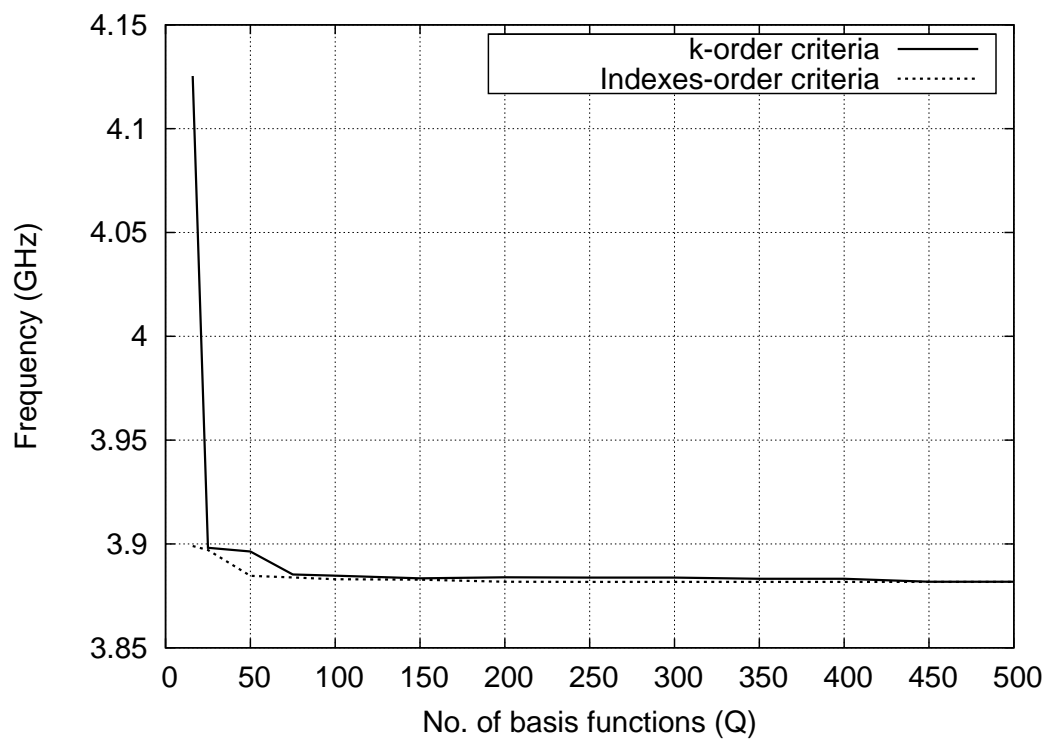

(a) $T M_{01}$ mode

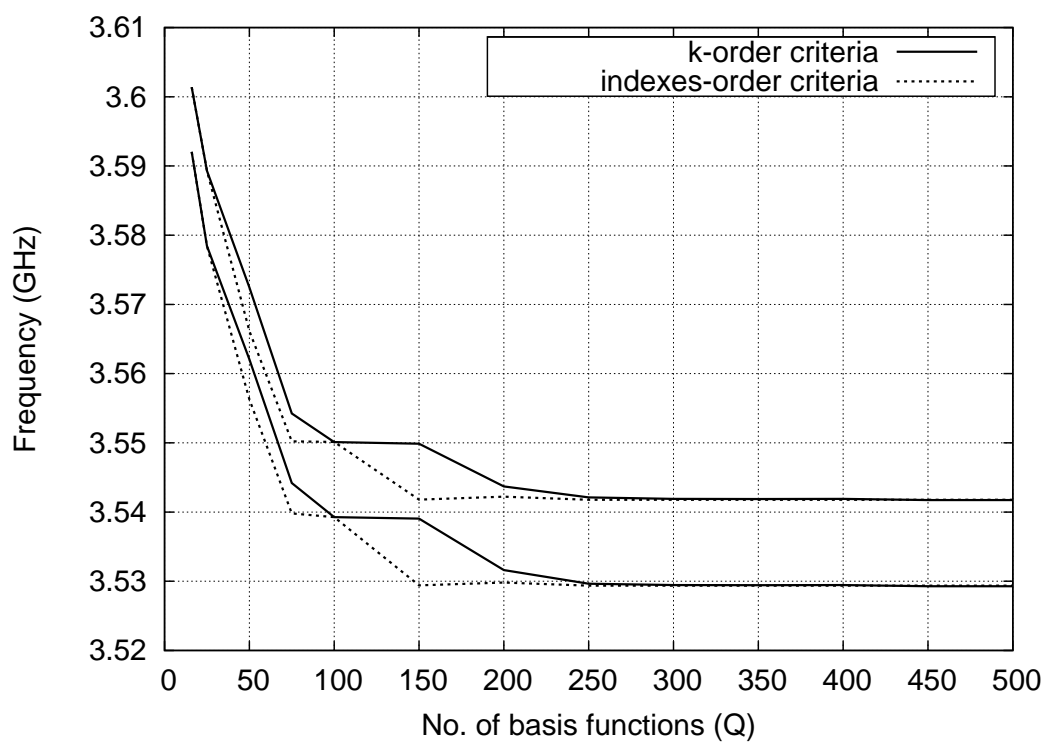

(b) $H E_{11}$ modes

Figure 5.17: Convergence study: Resonant frequencies as a function of the number of basis functions (Q) used by the MoM. Rectangular cavity dimensions: $a=25.4 \mathrm{~mm}$, $b=25.4 \mathrm{~mm}, c=23.368 \mathrm{~mm}$; DR: $h_{1}=8.4 \mathrm{~mm}, d=16.8 \mathrm{~mm}, \varepsilon_{r_{1}}=50$; DR support: $h_{2}=6.99 \mathrm{~mm}, \varepsilon_{r_{2}}=1$. 


\section{Chapter 6}

\section{Design of passive microwave filters based on dielectric resonators}

\subsection{Introduction}

During the last three decades, dielectric resonators have become a key element in many filtering applications [1-4]. Indeed, dielectric-loaded waveguide filters are frequently found in satellite and mobile communication systems, due to their advantages in terms of mass and volume reduction, low losses, and thermal stability. In the terrestrial mobile communication system industry [5], the cost of individual filters and the issue of mass production are crucial, whereas volume and weight are critical in satellite communications [7].

Since the early stages in the microwave technology history, electromagnetic waves propagation in dielectric media has been a key topic of research [110]. It was shown by Rayleigh in 1897 that an "infinitely" long cylinder of a dielectric material can serve as a guide for electromagnetic waves of certain frequencies. Also scientists like A. Sommerfeld in 1899 and D. Hondros in 1909 worked on the waves that can be guided by the discontinuity surface between two media having different electrical properties. D. Hondros and P. Debye centered their attention in studying dielectric rods to guide electromagnetic signals, obtaining a theoretical solution in 1910. Even G. C. Southworth at the Bell Telephone Laboratories started his research on waveguides considering dielectric media. He realized afterwards that in metal sheathed dielectric cylinders, the dielectric media was unnecessary and the wave was supported by the metal tube. He finally presented his work on hollow tube waveguides in 1936.

Apart from acting as electromagnetic guiding structures, dielectric materials can be also used as high-quality electrical resonators. In 1939, R. D. Richtmyer [8] introduced the term dielectric resonator (DR). He showed that unmetallized dielectric objects can function as electrical resonators. Nevertheless, the first activities on dielectric resonators did not occurred until the early 1960's when Okaya and Barash rediscovered DRs while working on high dielectric materials [9]. They provided the first analysis of modes and resonator design. During that decade, considerable efforts were devoted to the analysis of dielectric 
resonators [10-12]. In this area the most notable work was developed by Cohn and his coworkers, who performed an extensive theoretical and experimental study of the DR $[13,14]$. However, at that time the lack of suitable materials (due to their poor thermal stability) made impossible the development of practical components. The rutile ceramics $\left(\mathrm{TiO}_{2}\right)$ employed in the experiments had an isotropic dielectric constant in the order of 100 and a high-Q factor $(Q \sim 10000$ at $4 \mathrm{GHz})$. Unfortunately, they exhibited a very high temperature coefficient of resonant frequency $\left(\tau_{f} \sim 400 \mathrm{ppm} /{ }^{\circ} \mathrm{C}\right.$ ), thus causing too large resonant frequency changes, more than an order of magnitude too high for practical applications.

The breakthrough in ceramic materials technology occurred in the early 1970's when Masse and Pucel [15] developed the first temperature-stable and low-loss barium-tetratitanate ceramic $\left(\mathrm{BaTi}_{4} \mathrm{O}_{9}\right)$, which yielded $Q \sim 1300$ and $\tau_{f} \sim 5 \mathrm{ppm} /{ }^{\circ} \mathrm{C}$ at $9.6 \mathrm{GHz}$. They published the design of a microstrip bandpass filter using dielectric resonators made of this ceramic [16]. In subsequent years, new improved ceramic materials were developed which made possible to use DRs for microwave applications. In this scenario, the Murata Manufacturing Company played an important role since it was the first company that made commercially available temperature-stable ceramics [3].

In 1975, the first practical DR loaded microwave filter was reported by K. Wakino [17]. The advances in ceramic technology revived interest in dielectric resonators for a wide variety of microwave circuits $[18,19]$. New theoretical work and the use of DRs to design microwave filters expanded significantly during the 1980's. In 1982, a dual-mode axiallymounted dielectric resonator was reported by S. J. Fiedziuszko [20]. It nearly matched the performance of conventional dual-mode filters, and set the scene for the potential use of dielectric loaded multiplexers for space application. The structure is similar to the circular waveguide dual-mode filters with the exception of loading the circular cavities with dielectric pucks. The dual-degenerate $H E_{11 \delta}$ mode is used, and the couplings via resonators are performed via cruciform irises. This kind of filter has been successfully used in satellite communications applications. Subsequently, this work was extended by other authors [111-113]. Furthermore, as it has been explained in Chapter 5, during the 1980's complete studies on the DR's modal chart and the first rigorous full-wave analysis methods emerged [21-23].

During the last two decades, the new communication systems emerged demanding more stringent filter characteristics, have caused a significant progress in DR filter technology [4-6]. In particular, the rapid expansion of wireless communication industry has increased the demands for high-performance microwave filters and diplexers for both handsets and base station applications. In this area, coaxial cavity filters are commonly used due to their low cost and their spurious-free performance. Nevertheless, this kind of filters have limited quality factor values, and thus a different technology must be employed to match the new filtering requirements. In this context, the high-Q dielectric resonator filters have emerged as the baseline design for wireless base stations.

A typical dielectric resonator filter consists of a number of DRs mounted inside cavities machined in a metallic housing. One of the most used structures is the cylindrical suspended DR structure in a rectangular cavity (see in Fig. 6.1). The relative dielectric constant is typically between 20-80 and the DR is placed far from the enclosure. At the resonant frequency, 
most of the electromagnetic energy is stored within the dielectric. The enclosure stops radiation and since it is remote, the resonant frequency of the structure is largely controlled by the dimensions and permittivity of the dielectric. The quality factor $(Q)$ of the resonator cavity is dominated by the loss tangent of the ceramic material.

The dielectric resonator is placed inside the metal cavity by means of a dielectric support with a low dielectric permittivity. A layer of adhesive is used between the DR and the support, being both mounted inside the cavity employing a metallic or plastic screw. The support structure can degrade the quality factor if it is not properly designed. Since the resonant frequencies of each resonator are very sensitive to any change of the dielectric constant, tuning screws are always needed for readjustment due to manufacture tolerances on the dielectric constant of the DRs. The input and output RF energy are normally coupled to the filter through coaxial probes, while irises are used to provide the necessary coupling between resonators. The walls of the enclosure must be close enough to the dielectric resonator in order to the irises provide the necessary coupling. The enclosure is typically a square shape with a side width that is 1.5-1.7 times the diameter of the DR [5], however if one wants the enclosure not to have any influence in the Q-factor, it is known that the smaller dimension of the cavity should be 2-3 times the biggest dimension of the DR (normally the diameter) $[18,19]$. Indeed, despite the fact that the $\mathrm{Q}$ factor of the filter is mainly controlled by the loss tangent of the dielectric, in real components, it is reduced due to the losses in the metallic enclosure, support structure and tuning screws.

The first order resonant modes presented in Chapter 5 can be used as operating modes for dielectric resonator filters: single transverse transverse electric (TE) modes, single transverse transverse magnetic (TM) modes, dual hybrid (HE) modes, triple TE and triple TM modes. Single-mode DR filters operating in $T E_{01}$ modes are the most commonly used designs in wireless applications. The design offers the highest achievable filter $\mathrm{Q}$ in comparison with other modes of operation. The dual-mode filters present a reduced dimension compared to single-mode filters. Their design takes advantage of the dual-degenerate hybrid modes HE by placing the DR on the base of a housing. Thus, the hybrid modes are lowered in frequency and become the fundamental mode. A metallic disk is also placed on the top of the resonator to enhance its spurious response [114]. A design that also uses grounded DR is the dielectric combline filters reported in [32]. These are single-mode filters which operate in the TM mode. The dielectric rod has a height bigger than its diameter and it is positioned on the basis of the cavity, thus the TM mode becomes the fundamental one. Mixing metallic combline and dielectric loaded resonators, a filter presenting a high- $\mathrm{Q}$ and free-spurious performance can be designed. Another interesting design is the mixed modes cylindrical planar dielectric resonator filters [31]. In such a structure the resonators are excited in different modes ( $T E_{01}$ and $H E_{11}$ modes) to enhance the spurious performance of dual-mode filters operating only with hybrid modes. Finally, it is also remarkable that by using DR with different shapes, triple-mode [115] and quadruple-mode [116] filters can be developed.

In this chapter, different single-mode DR filters are presented. The dielectric puck loaded in rectangular waveguides or cavities is rigorously modelled by means of the State-Space Integral-Equation approach formulated in Chapter 5. We have implemented a software mod- 
ule to characterise the circuit building block represented by a rectangular cavity loaded with a dielectric puck. The resonator cavity may be opened through any of its lateral access ports and/or through its top surface. The efficient analysis of the dielectric resonator loaded cavity by means of the SS-IE approach is a key issue, since the dielectric resonator characterization requires the major part of the computational resources when one analyses more complex structures. Once the generalized admittance matrix (GAM) of this circuit building block is calculated, it may be connected to other circuit elements, in order to analyse different topologies of dielectric resonator filters. For such a purpose, we have integrated this software module in a Computer Aided Engineering (CAE) tool for the analysis and design of passive microwave and millimetre-waves components: FEST3D (Full-wave Electromagnetic Simulation Tool 3D) [81].

In the next section, we give a brief introduction to the modal electromagnetic solver FEST3D. We also describe how the algorithm developed in this thesis has been integrated into such tool. Finally, in the last section, different single-mode dielectric resonator bandpass filters, as well as a stopband filter, are presented. The bandpass filters may be designed by using dielectric-loaded rectangular cavities coupled through irises, or by placing the dielectric resonators inside a rectangular waveguide below cutoff. The results obtained by using the SS-IE approach integrated into FEST 3D are successfully compared with those obtained by the commercial software tool Ansoft HFSS [80].

\subsection{Integration into FEST3D}

As mentioned above, in this section we present a brief introduction to the CAE tool FEST3D (Full-wave Electromagnetic Simulation Tool 3D). The results shown in the next section have been achieved by integrating the new SS-IE formulation for the rigorous analysis of DRloaded rectangular cavities into FEST3D. For this reason, the aim of this section is to give a better understanding on how we have proceed to integrate the software module developed in this thesis into this general purpose electromagnetic solver. However, more detailed information about FEST3D may be obtained from [81].

FEST3D is a full-wave electromagnetic solver based on advanced modal algorithms for the analysis and design of passive microwave and millimetre-waves components for space and ground applications. This CAE tool is property of the European Space Agency (ESA). It is the result of several European R\&D projects that have been carried out during the last fifteen years, mainly under ESA contracts and a Research Training Network (2001-2004) funded by the European Commission V Framework programme. Several companies, universities and research institutions have been involved at different levels in the development of FEST3D: European Research and Technology Center from the European Space Agency (ESTEC/ESA, The Netherlands), Netherlands Organisation for Applied Scientific Research (TNO, The Netherlands), Aurora Software and Testing, S.L. (AURORASAT, Spain), Ingegneria dei Sistemi, S.p.A. (Italy), ITLink, S.r.L.(Italy), TESAT-SpaceCom Gmbh (Germany), École Polytechnique Fédérale de Lausanne (Switzerland), Technische Universität Darmstadt (Germany), Università di Pavia (Italy), Universidad Politécnica de Valencia and Universitat 
de València Estudi General. In this sense, it is remarkable that at the end of the last ESA contract devoted to further developments of FEST3D (September 2008), the European Space Agency transferred the exclusive distribution license of this CAE tool to the company AURORASAT. Thus, since then, it is this Spanish spin-off company who is in charge of the distribution, maintenance and development of FEST3D.

\subsubsection{Introduction to FEST3D.}

The software architecture of FEST3D can be split into three main blocks:

- The Graphical User Interface (GUI).

- The Optimiser and Tolerances module (OPT).

- The Electromagnetic Computational Engine (EMCE).

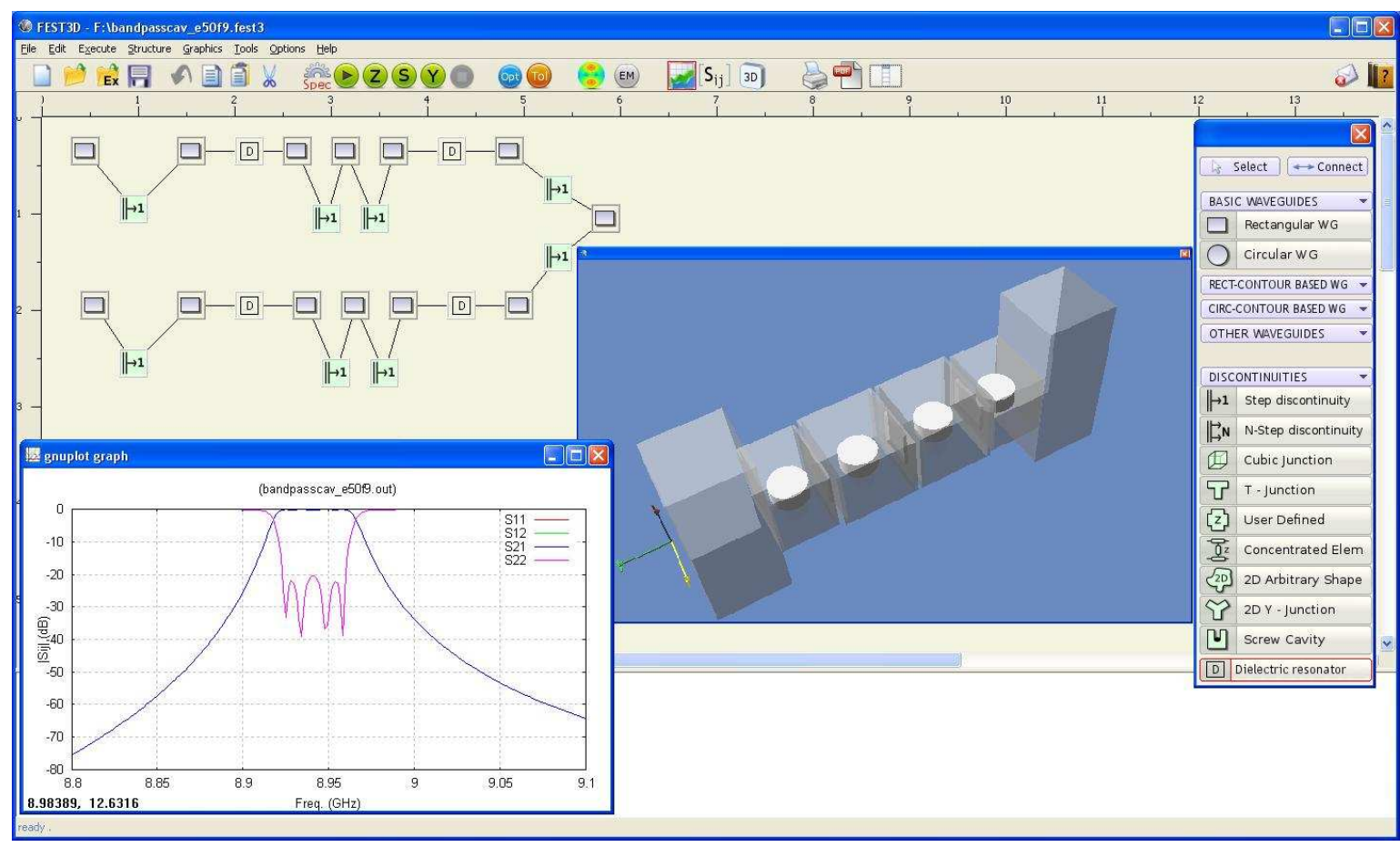

Figure 6.1: FEST3D GUI screenshot: A four-pole DR filter is simulated. The network circuit is represented in the cambas, while the real geometry is visualized. The electromagnetic response is shown by menas of the gnuplot program.

\section{The Graphical User Interface.}

The Graphical User Interface is a pure Java program. It offers user-friendly, intuitive CAElike interface to the user. It allows interactive edit, analysis (invoking the EMCE) and optimisation (invoking the OPT) of a microwave or millimetre wave circuit. The GUI supports the 
same set of components implemented in the EMCE, and lets the user easily create, modify, connect, delete, cut and paste components. Several canonical elements can be simulated by using the corresponding icons, and arbitrary 2D elements can be easily drawn by the user with the help of an special editor. It can start and control all the other tools (EMCE, OPT and all installed extensions) with a single click, and can graphically show the analysis results by driving the 'gnuplot' program. It also permits to visualize the real geometry of the circuit under analysis.

Moreover, the GUI allows to create easily a pdf file with all the information that the user desires to include about the circuit under analysis. It even offers the possibility to directly interact with the team responsible of the development of FEST3D by sending bug/technical reports via internet with simple clicks. In addition, it notifies to the user if there exist new versions of FEST3D which can be easily upgraded via internet.

The GUI offers to the user an easy-to-use and versatile interface to control all aspects of the optimiser (OPT). The user can choose with simple clicks which parameters of the components in the circuit he wants to optimise, and can watch their value changing during the optimisation procedure.

It is also remarkable that due to the used Java language and to the careful program design, new components (waveguides and junctions) and new extensions can be easily added to the GUI.

\section{The Optimiser and Tolerances module.}

The optimiser is a C++ program. Rather than focusing on a specialized optimisation algorithm, it exploits $\mathrm{C}++$ object-oriented features to offer a uniform interface where further algorithms can be easily integrated. The OPT is a standalone program, independent from both the GUI and the EMCE.

Both the EMCE and the OPT are built to be robust and behave predictably in case of errors. The EMCE detects and reports invalid circuit geometries, as well as inconsistent or unacceptably low numerical precision parameters. The OPT can detect such errors, and will "stay away" from points that cause the EMCE to fail, allowing the optimisation to proceed even in the case of extreme geometries (where a small perturbation can lead to an invalid geometry). The OPT supports multiple, weighted goal functions in the form of equalities or inequalities between one of the circuit scattering matrix elements $S_{m n}$ and a user-specified numerical function. The circuit to be optimised is - automatically - only simulated at the frequency points where at least one goal function is defined (each goal numerical function can be defined on a different set of frequency points).

The OPT also supports multiple, weighted constraints in the form of equalities or inequalities between a left and a right expression of the parameters being optimised. It is even possible to temporarily lock a parameter value or to set it to a formula (a function of the other parameters). The user can modify all aspects of optimisation (algorithms, parameters, goals and constraints) at any time that the OPT is not running. The OPT progress is shown in 
real-time using both text (error and parameters values) and graphical output using 'gnuplot'.

Moreover, FEST3D permits to perform tolerance analysis by using a specific module. The user can select any parameter of the simulated microwave device to perform a tolerance analysis. Then, independent Gaussian perturbations are applied to the selected parameters. The user can also choose the standard deviation of the Gaussian probability distribution.

\section{The Electromagnetic Computational Engine.}

The FEST3D EMCE is a mixed C++/Fortran77 program. On the one hand, it takes full advantage of the possibilities offered by object-oriented programming, on the other hand a significant portion of the computational intensive code is written in Fortran77, including efficient linear algebra algorithms. The EMCE architecture is component-based, encouraging assembling of possibly already existing software modules and also allowing upgrading and improvement with minimum impact on the application core. It is also remarkable that the EMCE can be compiled without any change on Linux, Windows and Sun operating systems.

The modal electromagnetic solver is based, mainly, in the following advanced numerical algorithms:

- The Integral Equation technique to characterize planar junctions between two or more waveguides described in $[62,117]$.

- The 2D version of the Boundary Integral-Resonant Mode Expansion (BI-RME) method for the electromagnetic analysis of $\mathrm{H}$-and E-plane arbitrary shaped structures $[66,67$, $69,70]$, as well as uniform waveguides with arbitrary shaped cross sections $[64,65]$.

- The 3D version of the BI-RME method for the electromagnetic analysis of rectangular cavities with cylindrical metallic posts $[74,75]$.

- Theory of cavities to characterise empty rectangular cavities with multiaperture [118], such as T-junctions or cubic-junctions.

The theory implemented in FEST3D supports the full-wave analysis of the following passive components:

- Rectangular, Circular, Elliptical, Coaxial, arbitrarily shaped waveguides;

- Infinite radiating arrays made of rectangular and circular waveguides; S- and U- bends;

- Planar transitions with multi-aperture able to connect a variety of waveguides;

- Cubic junctions; T junctions;

- Y junctions; 2-Dimensional Arbitrary junctions;

- Rectangular cavities loaded with cylindrical metallic posts.

FEST3D is based on a multimode equivalent network representation. This means that the microwave device under analysis is considered a circuit network made of different components of the list mentioned above, which are the network elements. Each network element in the microwave circuit is represented by a generalized impedance matrix (GIM), which 
gives the relationship between the electric and magnetic fields for each component in a multimode space. Therefore, taking advantage of the microwave network theory, the problem of analysing a big structure, e. g. a filter or a multiplexer, can be split into the analysis of elemental components. This fact makes it possible to develop a component-based software, as it was said before. Once the corresponding GIMs of each network element are calculated, the cascaded connection of these impedance matrices yields a matrix system representing the whole circuit. Then the input ports of the network structure are excited with the desired modes, and the scattering parameters are calculated by solving a banded matrix linear system. All of this is done for each frequency point of the frequency range selected by the user.

For an efficient analysis, the computation of the generalized impedance matrix of each network element has been split into two parts: the static and dynamic ones. In the static part, all the computations independent from the frequency are performed, and the variables needed to calculate later on the GIM of each element are stored. The GIMs, which depend on the frequency, are computed within the frequency loop, in the dynamic part. All this is possible thanks to the use of the integral equation technique and the BI-RME method mentioned above. On the one side, the integral equation formulation [62] used to characterise planar junctions between waveguides, permits to extract the frequency dependency from the kernel of the integral equation. Thus, the required infinite series in the kernel can be calculated outside the frequency loop. On the other side, the BI-RME method is used to analyse arbitrary shaped 2D components or rectangular cavities loaded with cylindrical posts. As explained in Chapter 3, the pole expansion of the generalized admittance matrix that characterise these components may be obtained in the frequency domain. Following this method, the resonant modes of the $2 \mathrm{D}$ arbitrary components and of cavities with posts are obtained through the solution of linear matrix eigenvalue problems in the static part. The eigenvalue and eigenvectors obtained in the static part are then used to calculate the GAM as a pole expansion (and consequently the GIM) in the dynamic part. In both approaches, a great amount of computation time is saved because a significant part of the needed computations are done only once, outside the frequency loop. Moreover, the solution of transcendental equations per frequency point is avoided. Hence, the electromagnetic behavior of the whole circuit can be solved in a wide and dense frequency range with a very reduced computational effort.

Furthermore, FEST3D provides a caching file service. For each simulation, the data calculated in the static part is stored in a separate cache file. In this way, if during a new simulation there is some element that is equal to an element analysed in one of the previous simulations, FEST3D takes the static data directly from the corresponding caching file, thus avoiding to repeat intensive computations performed before, and then saving computational effort.

FEST3D also supports symmetries: the user can specify global circuit symmetries (one or more of: constant width, constant height, all-centred, all-circular) and the EMCE will automatically switch to the set of waveguide modes appropriate for the specified set of symmetries. Since symmetries significantly lower the number of modes required to obtain an 
accurate result, this technique greatly reduces the computational time needed to simulate a circuit that has one or more of the above symmetries.

Finally, it is also remarkable that the EMCE provides specific modules in order to predict RF breakdown due to Multipactor and Corona phenomena. By using the electromagnetic fields computed by the electromagnetic solver, these modules can predict the breakdown voltage levels of the microwave device under analysis.

\subsubsection{Integration of the DR-loaded cavity module into FEST3D.}

In order to develop a software tool able to analyse dielectric resonator filters, the first step that we performed was the implementation of a software module to obtain the modal chart of a rectangular cavity loaded with a cylindrical dielectric resonator by means of the new SS-IE formulation. As it has been detailed in Chapter 3, to obtain the resonant modes, we have to solve the homogeneous eigenvalue problem defined in (3.42). Since the solution of such a problem implies intensive computations, we decided to use Fortran 77 as programming language. Once this first module was validated (see Chapter 5), we implemented the code to calculate the generalized admittance matrix (GAM) of the DR-loaded rectangular cavity as a pole expansion in the domain of the Laplace variable (see eq. (3.50)). As detailed in Chapter 3 , the resonator cavity may be opened by any of its lateral surfaces and/or the top cap.

At that point, we were able to obtain the electromagnetic response of a dielectric-loaded cavity resonator. However, in order to analyse more complex structures, such as filters, it was necessary to integrate the code developed into a general purpose electromagnetic solver that gave the possibility to connect it to other circuit elements. The choice was to integrate it into FEST3D, since it is an efficient full-wave electromagnetic solver that, like the formulation proposed in this thesis, make use of advanced modal techniques based on the integral equation technique. Due to the fact that the main program of FEST3D is written in C++ and it is an object-oriented code, in order to integrate the new software module it has been necessary to implement a C++ class that represents a new "FEST3D network element": a rectangular cavity loaded with a dielectric puck. This $\mathrm{C}++$ class is responsible of loading the parameters that describe the resonator cavity from a FEST input file, validating those parameters, connecting the cavity to other network elements attached to the access ports of the cavity, controlling the caching files, and finally calling the Fortran routines that calculate the generalized impedance matrix.

As it has been explained above, in FEST3D the computation of the GIM of each network element is split into the static and the dynamic part. In the case of the DR-loaded cavity, a Fortran77 subroutine is called in the static part to solve the eigenvalue problem (3.42), thus obtaining the resonant wavenumbers and the matrices $\mathbf{Y}^{\mathbf{A}}, \mathbf{Y}^{\mathbf{B}}$ and $\mathbf{y}^{(\mathbf{i})}$ (see eq. (3.51)) needed to calculate the GAM. In the dynamic part, another F77 subroutine to calculate the GAM is called. In the static part, the resonant wavenumbers and the matrices $\mathbf{Y}^{\mathbf{A}}, \mathbf{Y}^{\mathbf{B}}$ and $\mathbf{y}^{(\mathbf{i})}$ are conveniently stored and saved in the corresponding caching file. Thus, if FEST3D detects that there is a rectangular cavity loaded with a dielectric puck equal to other dielectric cavity resonator that has been already analysed in he current or previous simulations, it avoids to 


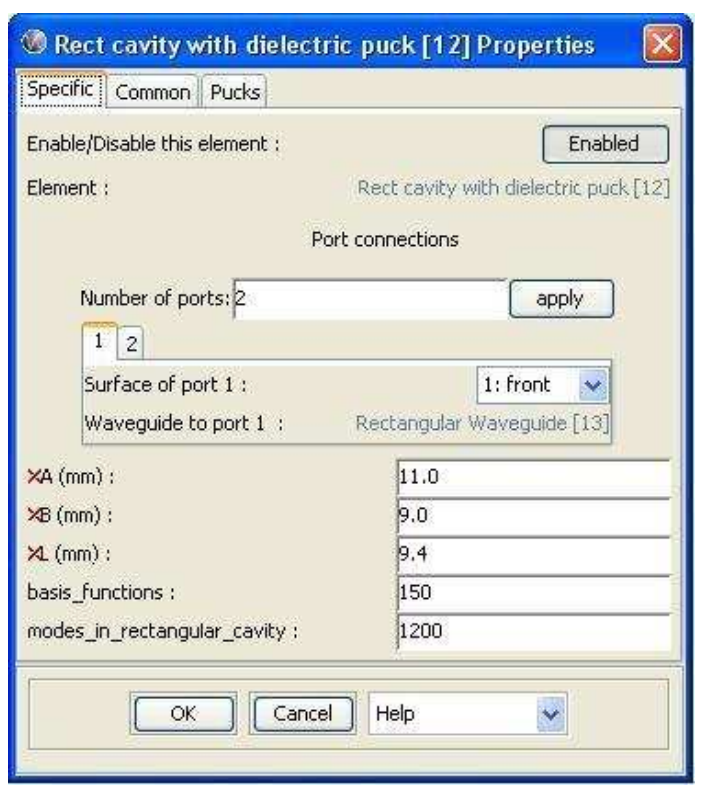

(a) This dialog window allows the introduction of the cavity dimensions, the number of basis functions and modes of the empty cavity used in the analysis. The surfaces of the rectangular cavities that are opened as access ports are also indicated in this window.

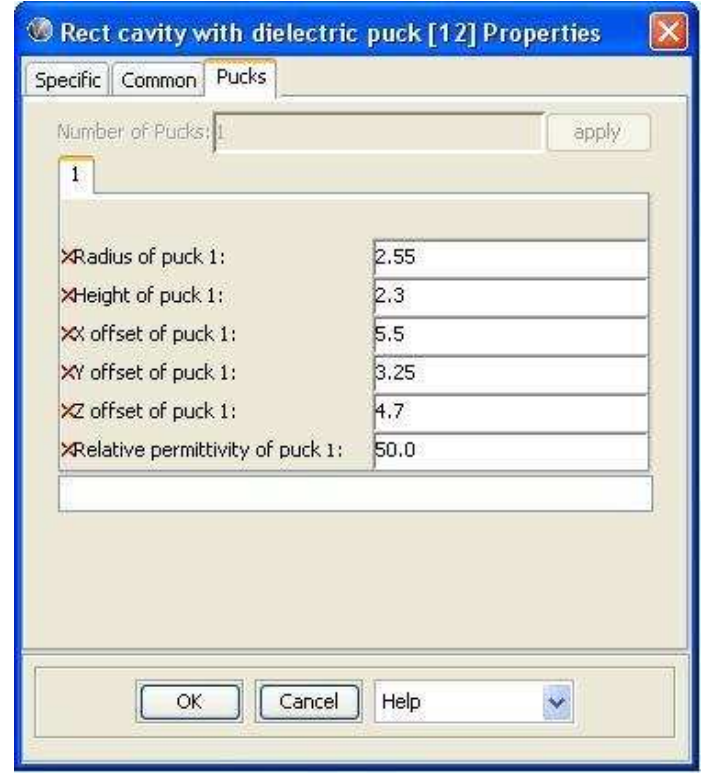

(b) This dialog window allows the user to introduce the dielectric resonator dimensions, the relative permittivity of the dielectric material and its position inside the rectangular cavity.

Figure 6.2: Dialog windows to introduce the data corresponding to the dielectric-loaded rectangular cavity.

solve again the eigenvalue problem (3.42) by copying the required data. For instance, when one analyses a symmetric DR filter with some repeated dielectric loaded cavities, FEST3D will perform the static computations only for each different cavity, thus saving computational resources. Due to the cache file service, this will even occur if the dielectric cavity resonator has been analysed in previous simulations.

In principle, the access ports of the dielectric-loaded rectangular cavity implemented must be rectangular waveguides with a cross section equal to the surface of the cavity. However, since FEST3D permits to analyse waveguides with zero length, the cavity resonator can be connected to any kind of circuit elements, such as any kind of iris or T-junctions, through a zero length rectangular waveguides.

Finally, the new FEST3D network element has been integrated in the graphical user interface. Thus, the user can easily introduce the data that characterise the dielectric-loaded cavity resonator and visualize the real device under analysis. Fig. 6.2 show the windows where the user introduces all the needed parameters. 


\subsection{Bandpass and bandstop dielectric resonator filters}

In this section, the CAE tool FEST3D upgraded with the software module developed in the present thesis is used for the design of different dielectric resonator filters in rectangular waveguide. Firstly, we present some prototype structures that are used as basis for the singlemode DR filters designed. Afterwards, the analysis of different bandpass and bandstop filters by means of the new software tool is successfully compared with the results obtained with the well-known electromagnetic solver Ansoft HFSS [80]. Since HFSS is based on the FiniteElement Method (FEM), which is completely different from the SS-IE formulation presented in this work and the modal techniques used in FEST3D, it represents a good reference to validate the software developed in this thesis.

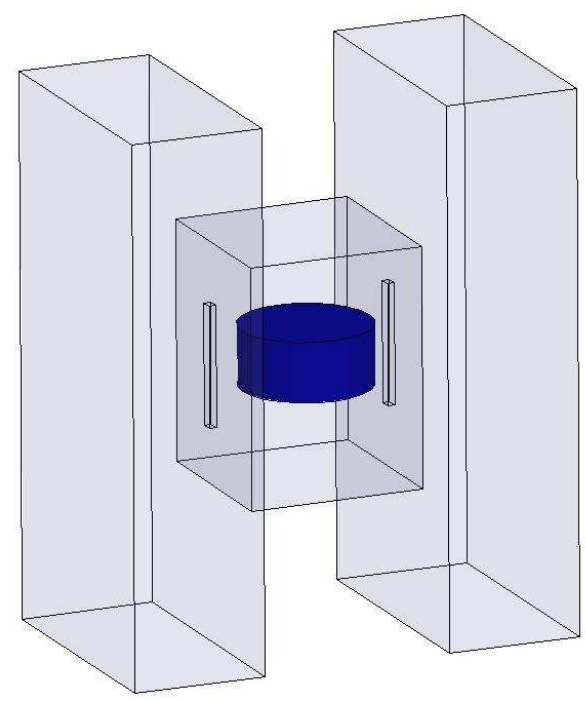

Figure 6.3: Bandpass structure using dielectric resonators in a rectangular waveguide enclosure.

The bandpass prototype structure to be described is made of a rectangular cavity loaded with a dielectric puck connected through rectangular irises at both ends to propagating rectangular waveguides. The rotation axis of the dielectric cylinder is oriented parallel to the largest side of the input/output waveguides as shown in Fig. 6.3. When the propagating waveguides are excited on their fundamental mode $\left(T E_{10}\right)$, the reactangular cavity containing the DR is under cutoff, therefore in the absence of the dielectric body, no energy would be transmitted between the propagating waveguides. Nevertheless, in the prototype structure, the input waveguide excites through the iris the electromagnetic fields in the cavity, which decay exponentially with the distance from the iris. These evanescent fields excite, via the magnetic field, the fundamental $T E_{01}$ mode in the dielectric resonator, and then the energy is transmitted to the output waveguide.

Table 6.1 shows the dimensions of the dielectric-loaded reactangular cavity and the irises of the bandpass prototype analysed. In that table, $w, h$ and $t$ mean, respectively, the width, 


\begin{tabular}{||l|ccc||}
\hline \hline Rectangular Cavity & $a(\mathrm{~mm})$ & $b(\mathrm{~mm})$ & $c(\mathrm{~mm})$ \\
& 13.5 & 17.88 & 13.2 \\
\hline Rectangular Iris & $w(\mathrm{~mm})$ & $h(\mathrm{~mm})$ & $t(\mathrm{~mm})$ \\
& 1.0 & 9.0 & 0.5 \\
\hline Dielectric Resonator & $d(\mathrm{~mm})$ & $h_{1}(\mathrm{~mm})$ & $\varepsilon_{r_{1}}$ \\
& 9.84 & 4.43 & 44 \\
\hline \hline
\end{tabular}

Table 6.1: Bandpass prototype parameters

the height and the thickness of the rectangular iris. The DR is centered with respect to the bottom surface of the rectangular cavity and its base is placed at a height $h_{2}=6.73 \mathrm{~mm}$. The input/output ports are WR-159 (40.39 ×20.193 mm) waveguides.

To characterise the dielectric loaded cavity, we have used 250 basis functions, 1000 resonant modes of the rectangular cavity and 20 accessible modes in the apertures. Moreover, to characterise the junctions between the rectangular waveguides by means of the integral equation technique, we have employed 20 accessible modes, 150 basis functions and 600 modes in the series of the integral equation kernel. The electromagnetic response of the prototype structure presented in Table 6.1 is shown in Fig. 6.4. Two peaks of the scattering parameter $\left|S_{12}\right|$ can be observed at $5.195 \mathrm{GHz}$ and $6.429 \mathrm{GHz}$ corresponding to the excitation of the fundamental mode $T E_{01}$ and the hybrid mode $H E_{11}$ of the DR.

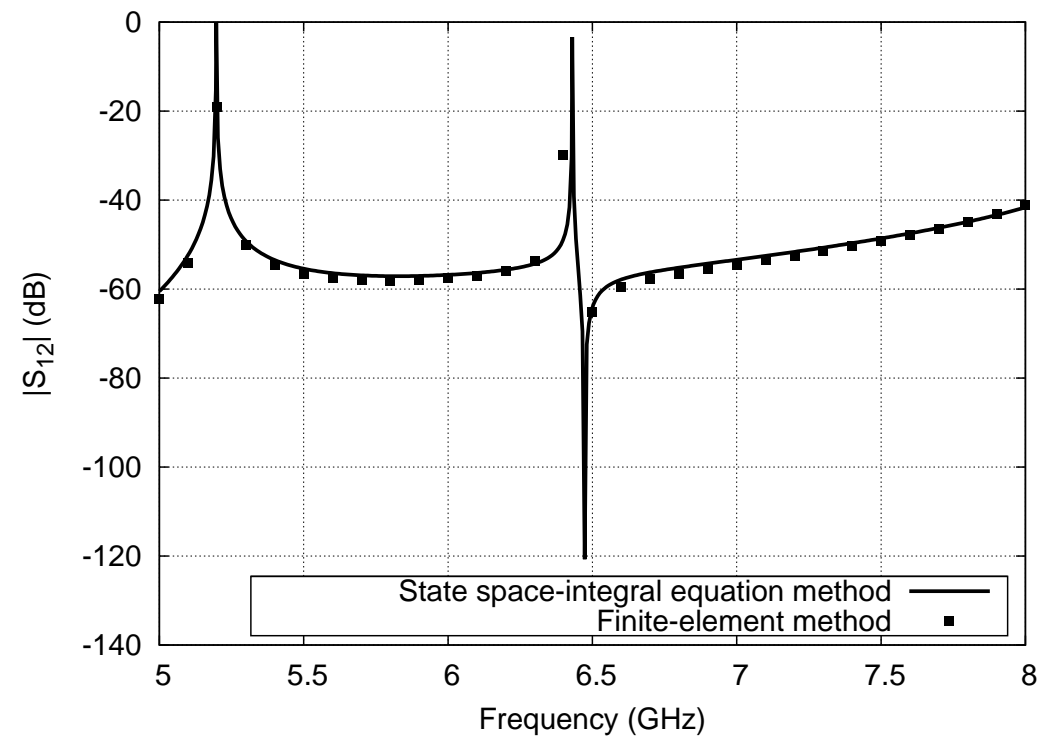

Figure 6.4: Electromagnetic response of the bandpass prototype described in Table 6.1. The results are compared with the FEM electromagnetic solver Ansoft HFSS.

Next, we can use the idea behind the previous bandpass prototype structure to design a bandpass filter. We can connect different dielectric-loaded rectangular cavities to each other, through rectangular irises, to design a bandpass filter. Thus, the evanescent fields that have 


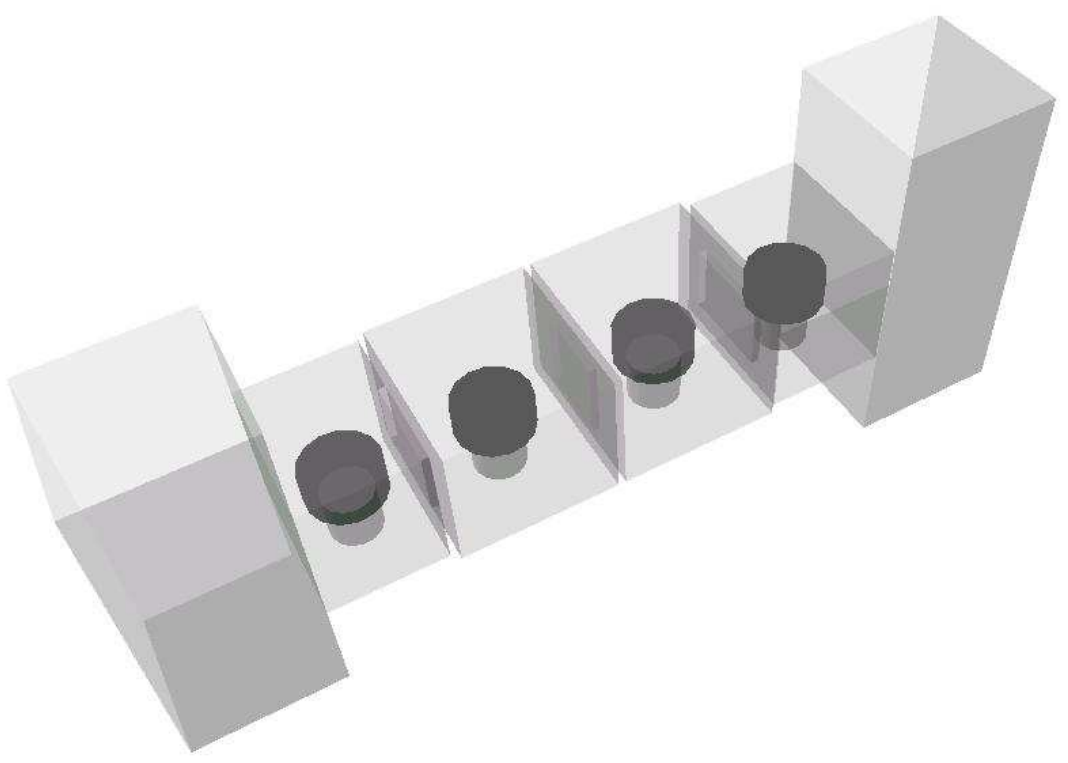

Figure 6.5: Four-pole band-pass filter based on dielectric resonators in rectangular cavities connected through rectangular irises.

excited the dielectric resonator in the prototype, will now excite the DR in the nearest cavity resonator and the signal will be transmitted through the next dielectric resonators to the output propagating waveguide. We have employed the new software tool to design a fourpole band-pass filter based on dielectric loaded rectangular cavity resonators (see Fig. 6.5). The dielectric support of the resonators is assumed to have a relative permittivity $\varepsilon_{r_{2}}=1$, and a height of $h_{2}=3.25 \mathrm{~mm}$. The DRs are centered with respect to the base of their rectangular enclosures. The input and output ports of the filter are standard WR-90 waveguides and are directly coupled to the first and the last cavities. The designed bandpass filter is symmetric in the propagating direction, and the rest of its geometrical parameters are included in Table 6.2 .

\begin{tabular}{||c|ccc||}
\hline \hline Rectangular Cavities & $a(\mathrm{~mm})$ & $b(\mathrm{~mm})$ & $c(\mathrm{~mm})$ \\
\hline $1 \& 4$ & 10.0 & 9.0 & 7.7 \\
$2 \& 3$ & 11.0 & 9.0 & 10.4 \\
\hline Rectangular Irises & $w(\mathrm{~mm})$ & $h(\mathrm{~mm})$ & $t(\mathrm{~mm})$ \\
\hline $1 \& 3$ & 6.08 & 4.43 & 0.5 \\
2 & 4.85 & 5.27 & 0.5 \\
\hline Dielectric Resonators & $d(\mathrm{~mm})$ & $h_{1}(\mathrm{~mm})$ & $\varepsilon_{r_{1}}$ \\
\hline & 5.10 & 2.3 & 50 \\
\hline \hline
\end{tabular}

Table 6.2: Dimensions of a four-pole filter centered at $8.94 \mathrm{GHz}$. It is based on dielectricloaded cavities coupled through rectangular irises. The dielectric support of the resonators is assumed to have a relative permittivity $\varepsilon_{r_{2}}=1$, and a height of $h_{2}=3.25 \mathrm{~mm}$. 
In order to analyse the filter described in Table 6.2, we have used 150 basis functions to characterise the dielectric resonators, 700 resonant modes of the rectangular cavities and 50 accessible modes in the apertures. In addition, to characterise the junctions between the rectangular waveguides by means of the integral equation technique, we have employed 50 accessible modes, 150 basis functions and 500 modes in the series of the integral equation kernel. We have simulated this filter with an Intel Core $2 \mathrm{Duo}$ at $1.83 \mathrm{GHz}$, and the required computational effort has been of $49 \mathrm{~s}$ in the frequency-independent part, and of $0.4 \mathrm{~s}$ per frequency point. The filter is centered at $8.94 \mathrm{GHz}$ with a bandwidth of $37 \mathrm{MHz}$. The results obtained with the new code developed are again successfully compared with those provided by the commercial code Ansoft HFSS in Fig. 6.6 and 6.7. The out of band response is also shown in Fig. 6.8.

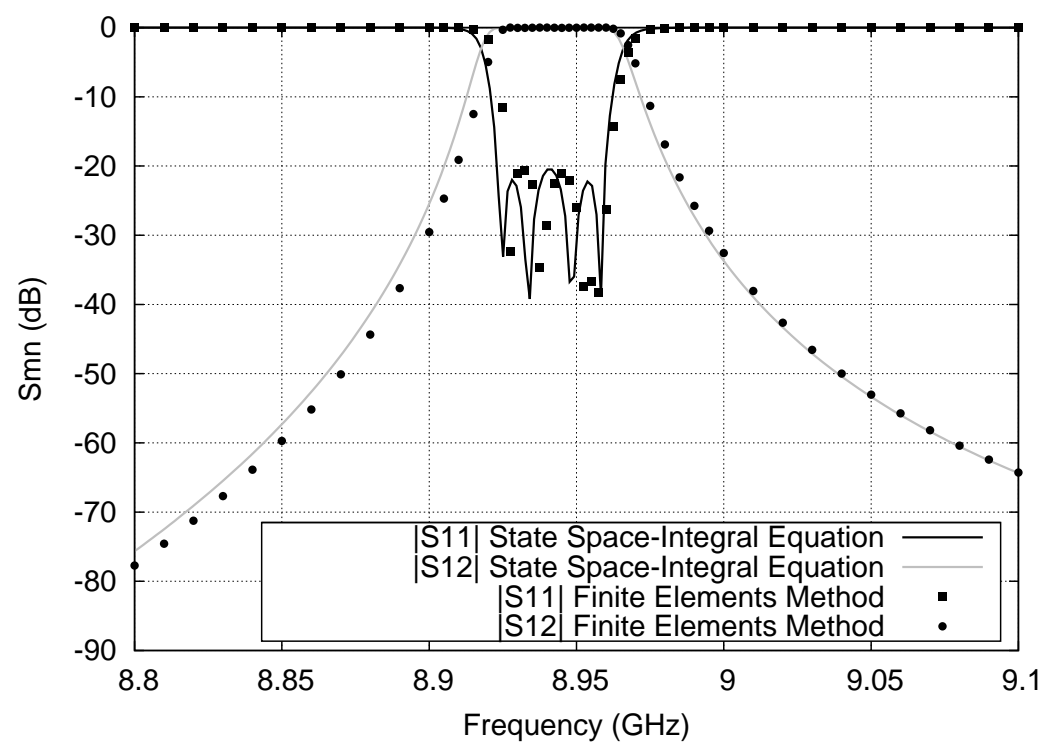

Figure 6.6: Electromagnetic response of the bandpass filter described in Table 6.2. Comparison between the state space-integral equation method and the Finite-Element Method (Ansoft HFSS).

The next bandpass filter designed using dielectric-loaded cavity resonators has a bandwidth of $40 \mathrm{MHz}$ and its pass-band is centered at $5 \mathrm{GHz}$. The input/output ports are WR-187 $(47.55 \times 22.147 \mathrm{~mm})$ rectangular waveguides, which are directly coupled to the cavity resonators. The dielectric supports have a height of $h_{2}=3.25 \mathrm{~mm}$, and a relative permittivity of $\varepsilon_{r_{2}}=1$. The rest of parameters are shown in Table 6.3. In this case, to obtain the electromagnetic response of the filter designed, we have needed: 250 basis functions in the DRs; 600 resonant modes in the reactangular cavities; 30 accessible modes in the apertures. To characterise the planar junctions between the rectangular waveguides, we have used 30 accessible modes, 100 basis functions and 400 modes in the series of the integral equation kernel. The computational time used in the analysis of such a filter has been $45 \mathrm{~s}$ in the static 


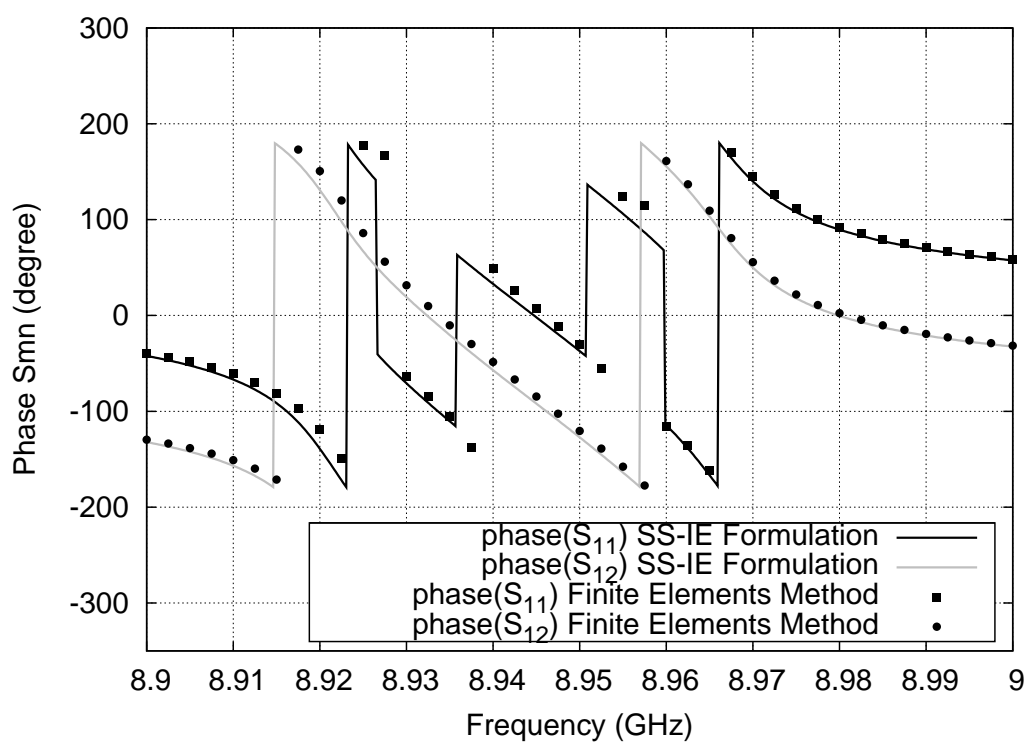

Figure 6.7: Electromagnetic response of the band-pass filter described in Table 6.2. Comparison of the scattering parameters phases between the state-space integral-equation method and Ansoft HFSS.

part and $0.12 \mathrm{~s}$ per frequency point in the dynamic part with an Intel Core 2 Duo @ $1.83 \mathrm{GHz}$ processor. The comparison of our results with the FEM-based electromagnetic solver Ansoft HFSS is presented in Fig. 6.9.

\begin{tabular}{||c|ccc||}
\hline \hline Rectangular Cavities & $a(\mathrm{~mm})$ & $b(\mathrm{~mm})$ & $c(\mathrm{~mm})$ \\
\hline $1 \& 4$ & 13.5 & 17.88 & 13.2 \\
$2 \& 3$ & 17.18 & 17.88 & 16.6 \\
\hline Rectangular Irises & $w(\mathrm{~mm})$ & $h(\mathrm{~mm})$ & $t(\mathrm{~mm})$ \\
\hline $1 \& 3$ & 6.4 & 8.68 & 0.5 \\
2 & 6.12 & 8.85 & 0.5 \\
\hline Dielectric Resonators & $d(\mathrm{~mm})$ & $h_{1}(\mathrm{~mm})$ & $\varepsilon_{r_{1}}$ \\
\hline & 9.84 & 4.43 & 44 \\
\hline
\end{tabular}

Table 6.3: Dimensions of a four-pole filter centered at $5.0 \mathrm{GHz}$. It is based on dielectricloaded cavities coupled through rectangular irises. The dielectric support of the resonators is assumed to have a relative permittivity $\varepsilon_{r_{2}}=1$, and a height of $h_{2}=6.73 \mathrm{~mm}$.

Fig. 6.10 shows the electromagnetic response of the third bandpass filter designed using dielectric-loaded cavity resonators coupled via rectangular irises. In this case, the input/output ports are WR-90 $(19.05 \times 9.525 \mathrm{~mm})$ rectangular waveguides, which are directly coupled to the cavity resonators. The dielectric supports are centered with respect to the base of the rectangular enclosures, having a height of $h_{2}=2.975 \mathrm{~mm}$, and a relative permittivity of $\varepsilon_{r_{2}}=1$. The rest of parameters are shown in Table 6.4. The four-pole filter 


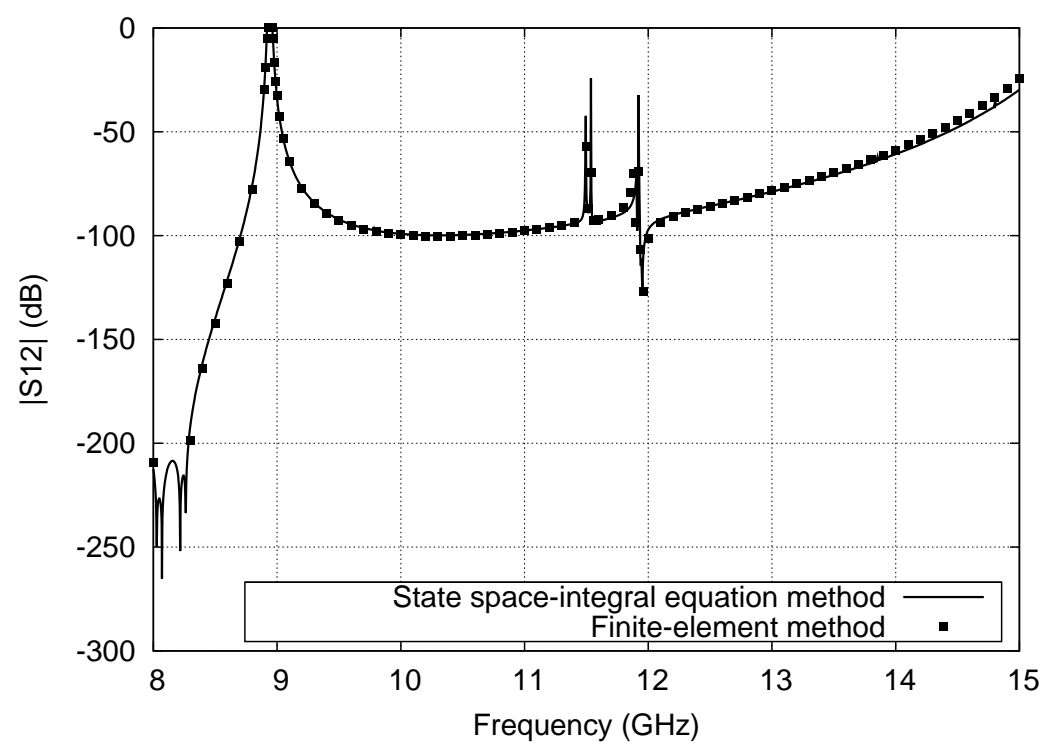

Figure 6.8: Electromagnetic response of the band-pass filter described in Table 6.2. Comparison in the out of band range between the state-space integral-equation method and Ansoft HFSS.

has a bandwidth of $78 \mathrm{MHz}$ and its pass-band is centered at $10.74 \mathrm{GHz}$. We have used 250 basis functions in the DRs, 600 resonant modes in the reactangular cavities and 25 accessible modes in the apertures. To characterise the planar junctions between the rectangular waveguides, we have used 25 accessible modes, 100 basis functions and 450 modes in the series of the integral equation kernel. To simulate the filter $44 \mathrm{~s}$ have been used in the static part, while $0.1 \mathrm{~s}$ per frequency point were employed in the dynamic part using the same computer than in the previous test cases.

\begin{tabular}{||c|ccc||}
\hline \hline Rectangular Cavities & $a(\mathrm{~mm})$ & $b(\mathrm{~mm})$ & $c(\mathrm{~mm})$ \\
\hline $1 \& 4$ & 6.14 & 8.13 & 7.25 \\
$2 \& 3$ & 7.99 & 8.13 & 7.56 \\
\hline Rectangular Irises & $w(\mathrm{~mm})$ & $h(\mathrm{~mm})$ & $t(\mathrm{~mm})$ \\
\hline $1 \& 3$ & 2.8 & 4.4 & 0.5 \\
2 & 2.3 & 4.1 & 0.5 \\
\hline Dielectric Resonators & $d(\mathrm{~mm})$ & $h_{1}(\mathrm{~mm})$ & $\varepsilon_{r_{1}}$ \\
\hline & 4.84 & 2.18 & 40 \\
\hline \hline
\end{tabular}

Table 6.4: Dimensions of a four-pole filter centered at $10.74 \mathrm{GHz}$. It is based on dielectricloaded cavities coupled through rectangular irises. The dielectric support of the resonators is assumed to have a relative permittivity $\varepsilon_{r_{2}}=1$, and a height of $h_{2}=2.975 \mathrm{~mm}$.

Up to now, we have presented different bandpass filters based on dielectric-loaded rectangular cavities where the coupling between cavity resonators is performed by means of 


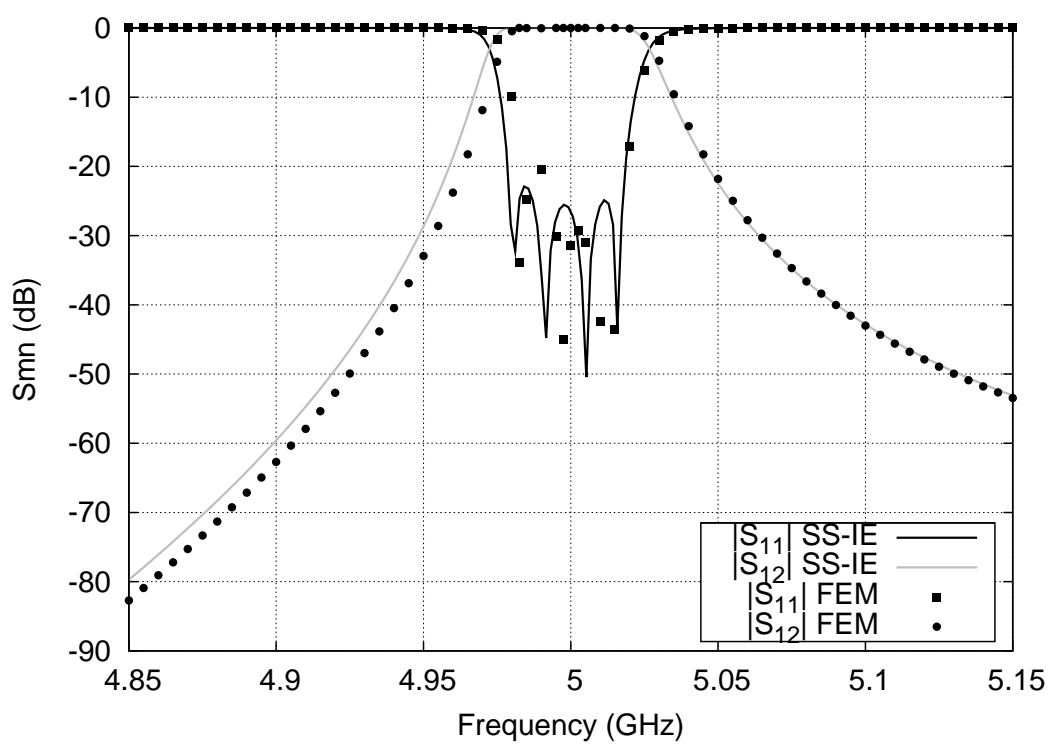

Figure 6.9: Electromagnetic response of the bandpass filter described in Table 6.3. Comparison between the state space-integral equation method and the Finite-Element Method (Ansoft HFSS).

rectangular irises. However, this coupling between resonators can also be achieved via direct coupling by placing the dielectric resonators in a cutoff waveguide as it is shown in Fig. 6.11. Next, we describe three different four-pole filters based on dielectric resonators in rectangular waveguides below cutoff.

The first DR filter designed of this kind consists of a rectangular waveguide housing $(9.19 \times 9.19 \mathrm{~mm})$ loaded with four cylindrical dielectric resonators, which is connected to WR-90 $(19.05 \times 9.525 \mathrm{~mm})$ waveguides acting as input/output ports. The dielectric resonators are centered with respect to the axis of propagation and the height of the four dielectric supports is $h_{2}=3.59 \mathrm{~mm}$. The rest of parameters are shown in Table 6.5. The pass-band of the filter is centered at $11.98 \mathrm{GHz}$. We have used 200 basis functions in the DRs, 700 resonant modes in the rectangular cavities and 20 accessible modes in the apertures. To characterise the planar junctions between the rectangular waveguides, we have used 20 accessible modes, 40 basis functions and 200 modes in the series of the integral equation kernel. To simulate the filter, $21.5 \mathrm{~s}$ have been used in the static part, while $0.01 \mathrm{~s}$ per frequency point were employed in the dynamic part using the same computer than in the previous test cases. The electromagnetic response obtained with our code is successfully compared with the results of Ansoft HFSS as it is shown in Fig. 6.12.

The input/output ports of the second dielectric-loaded waveguide filter are WR-112 (28.5x $12.624 \mathrm{~mm}$ ) waveguides. The four dielectric pucks are placed, centered with respect to the axis of propagation, in a rectangular waveguide housing whose dimensions are $12.25 \times 12.25$ $\mathrm{mm}$. The rest of parameters are shown in Table 6.6. In order to obtain the electromagnetic response of the filter, we have used 250 basis functions in the DRs, 800 resonant modes in 


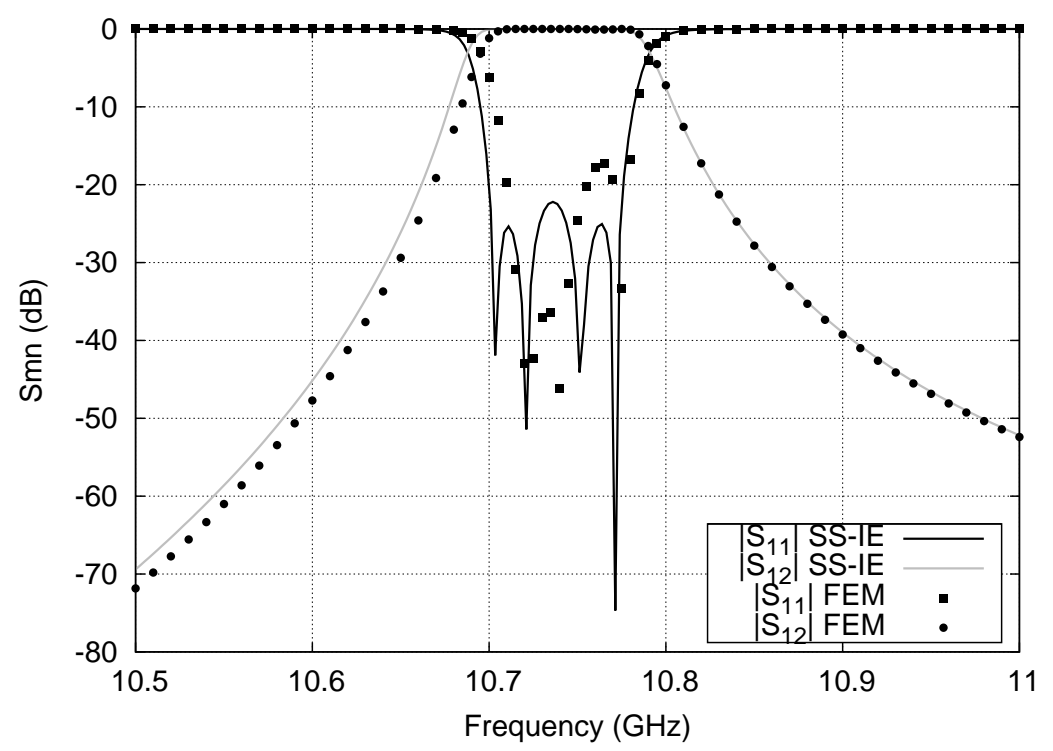

Figure 6.10: Electromagnetic response of the bandpass filter described in Table 6.4. Comparison between the state space-integral equation method and the Finite-Element Method (Ansoft HFSS).

\begin{tabular}{||l|ccc||}
\hline \hline Lengths between DRs & $L_{1}(\mathrm{~mm})$ & $L_{2}(\mathrm{~mm})$ & $L_{3}(\mathrm{~mm})$ \\
\hline & 3.34 & 11.04 & 12.13 \\
Dielectric Resonators & $d(\mathrm{~mm})$ & $h_{1}(\mathrm{~mm})$ & $\varepsilon_{r_{1}}$ \\
\hline & 4.24 & 2.01 & 38 \\
\hline \hline
\end{tabular}

Table 6.5: Dimensions of a four-pole filter at $11.98 \mathrm{GHz}$ based on dielectric-loaded rectangular waveguide below cutoff. The dielectric support of the resonators is assumed to have a relative permittivity $\varepsilon_{r_{2}}=1$, and a height of $h_{2}=3.59 \mathrm{~mm}$.

the rectangular cavities and 30 accessible modes in the apertures. To characterise the planar junctions between the rectangular waveguides, we have used 30 accessible modes, 60 basis functions and 300 modes in the series of the integral equation kernel. To simulate the whole structure, $28 \mathrm{~s}$ have been used in the static part, while $0.04 \mathrm{~s}$ per frequency point have been employed in the dynamic part using an Intel Core 2 Duo @ $1.83 \mathrm{GHz}$ processor. The passband of the filter is centered at $9 \mathrm{GHz}$. Our results are successfully compared with those obtained by using Ansoft HFSS as it is shown in Fig. 6.13.

The design parameters of the last dielectric-loaded waveguide filter are shown in Table 6.7. It consists of a rectangular waveguide $(27.56 \times 27.56 \mathrm{~mm})$ loaded with four cylindrical dielectric resonators, which is connected to WR-229 $(58.17 \times 29.08 \mathrm{~mm})$ waveguides acting as input/output ports. The dielectric resonators are centered with respect to the axis of propagation and the height of the four dielectric supports is $h_{2}=10.98 \mathrm{~mm}$. The pass-band of the filter is centered at $3.99 \mathrm{GHz}$. We have used 150 basis functions in the DRs, 500 resonant 


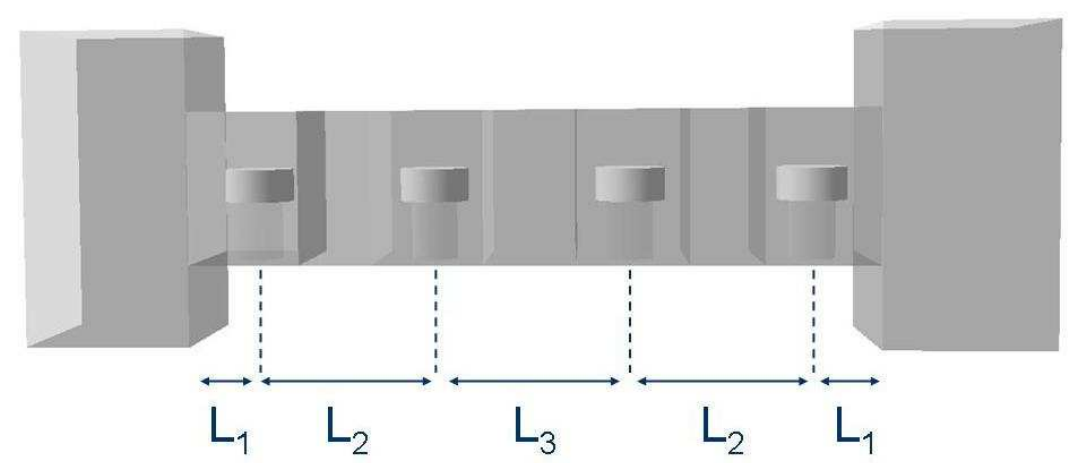

Figure 6.11: Bandpass filter in rectangular waveguide. The dielectric resonators are directly coupled.

\begin{tabular}{||l|ccc||}
\hline \hline Lengths between DRs & $L_{1}(\mathrm{~mm})$ & $L_{2}(\mathrm{~mm})$ & $L_{3}(\mathrm{~mm})$ \\
\hline & 4.105 & 14.72 & 16.17 \\
Dielectric Resonators & $d(\mathrm{~mm})$ & $h_{1}(\mathrm{~mm})$ & $\varepsilon_{r_{1}}$ \\
\hline & 4.92 & 2.33 & 50 \\
\hline \hline
\end{tabular}

Table 6.6: Dimensions of a four-pole filter at $9 \mathrm{GHz}$ based on dielectric-loaded rectangular waveguide below cutoff. The dielectric support of the resonators is assumed to have a relative permittivity $\varepsilon_{r_{2}}=1$, and a height of $h_{2}=4.96 \mathrm{~mm}$.

modes in the reactangular cavities and 50 accessible modes in the apertures. To characterise the planar junctions between the rectangular waveguides, we have used 50 accessible modes, 70 basis functions and 300 modes in the series of the integral equation kernel. To simulate the filter, $14 \mathrm{~s}$ have been used in the static part, while $0.14 \mathrm{~s}$ per frequency point were employed in the dynamic part using the same computer than in the previous test cases. The electromagnetic response of the filter is shown in Fig. 6.14.

\begin{tabular}{||c|ccc||}
\hline \hline Lengths between DRs & $L_{1}(\mathrm{~mm})$ & $L_{2}(\mathrm{~mm})$ & $L_{3}(\mathrm{~mm})$ \\
\hline & 8.55 & 31.1 & 33.74 \\
Dielectric Resonators & $d(\mathrm{~mm})$ & $h_{1}(\mathrm{~mm})$ & $\varepsilon_{r_{1}}$ \\
\hline & 11.8 & 5.6 & 44 \\
\hline \hline
\end{tabular}

Table 6.7: Dimensions of a four-pole filter at $3.99 \mathrm{GHz}$ based on dielectric-loaded rectangular waveguide below cutoff. The dielectric support of the resonators is assumed to have a relative permittivity $\varepsilon_{r_{2}}=1$, and a height of $h_{2}=10.98 \mathrm{~mm}$.

The next structures to be considered are shown in Figs. 6.15(a) and 6.15(b). This kind of bandstop structures in waveguide technology was reported by Ren [119]. It is realized by coupling the dielectric resonator to a propagating waveguide. The resonators are located 


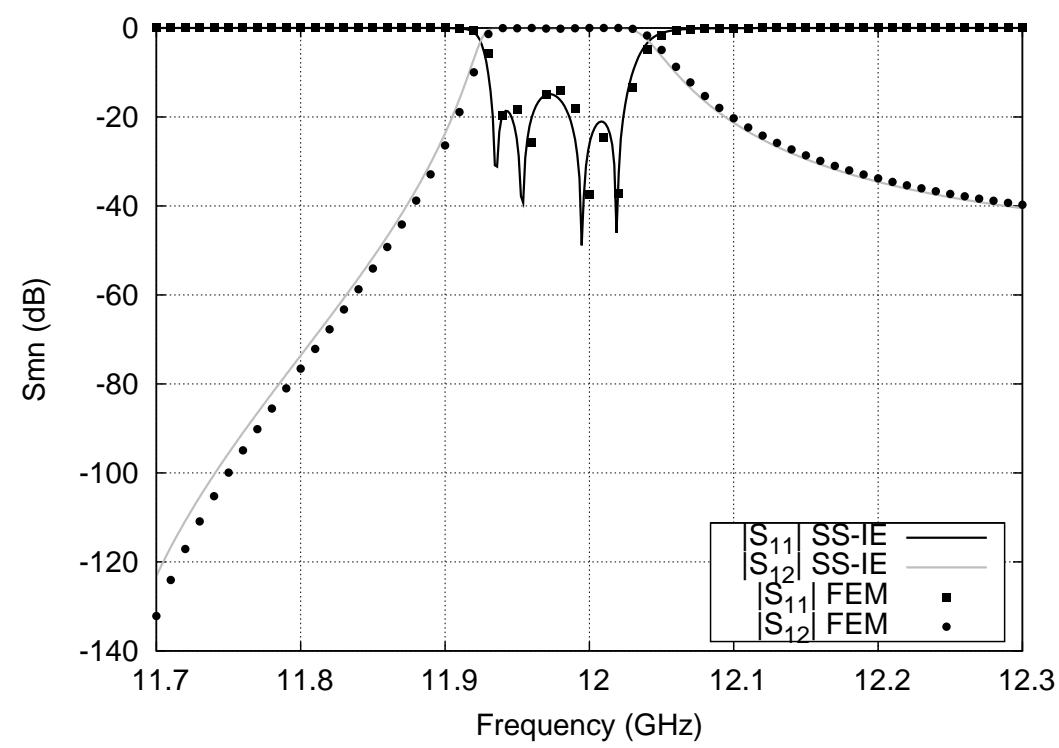

Figure 6.12: Electromagnetic response of the dielectric-loaded rectangular waveguide bandpass filter described in Table 6.5.

in their own metal enclosures, and the coupling to the main waveguide is achieved through apertures in the waveguide walls. This configuration provides isolation for individual DR and reduces their perturbation of the waveguide. Like in the case of the bandpass prototype, the rectangular cavity containing the resonator is below cutoff. When the $T E_{10}$ mode is excited in the propagating waveguide, the magnetic field of that mode couples with the magnetic field of the fundamental $T E_{01}$ mode in the dielectric resonator. Thus, the energy is absorbed by the DR and reflected. In the structure shown in Fig. 6.15(a), the coupling is performed via the $H_{x}$ component of the fundamental mode in the propagating waveguide, whereas in the case of Fig. 6.15(b), it is performed via the $H_{z}$ component of the same mode.

\begin{tabular}{||l|ccc||}
\hline \hline Rectangular Cavity & $a(\mathrm{~mm})$ & $b(\mathrm{~mm})$ & $c(\mathrm{~mm})$ \\
\hline & 10.0 & 9.0 & 7.7 \\
Rectangular Iris & $w(\mathrm{~mm})$ & $h(\mathrm{~mm})$ & $t(\mathrm{~mm})$ \\
\hline & 1.0 & 8.0 & 0.35 \\
Dielectric Resonator & $d(\mathrm{~mm})$ & $h_{1}(\mathrm{~mm})$ & $\varepsilon_{r_{1}}$ \\
\hline & 5.10 & 2.3 & 50 \\
\hline \hline
\end{tabular}

Table 6.8: Bandstop prototypes parameters

The analysed bandstop prototypes consist of a WR-90 $(22.86 \times 10.16 \mathrm{~mm})$ waveguide connected to a rectangular cavity loaded with a dielectric resonator through a rectangular iris. The dielectric support is assumed to have unitary relative dielectric permittivity with a height $h_{2}=3.25 \mathrm{~mm}$, the rest of geometry parameters are shown in Table 6.8. Both the $H_{x}$ and the $H_{z}$ configurations have been simulated (see Fig. 6.15(a) and Fig. 6.15(b), 


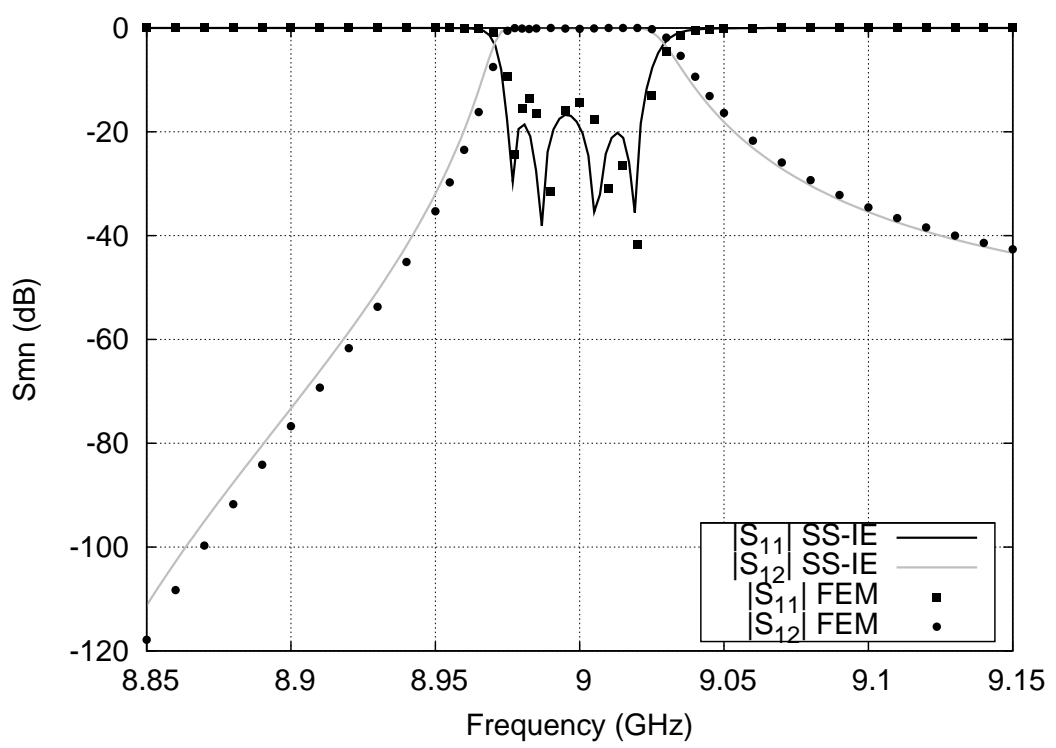

Figure 6.13: Electromagnetic response of the dielectric-loaded rectangular waveguide bandpass filter described in Table 6.6.

respectively).

In order to obtain the electrical response of these structures, we have employed 150 basis functions, 900 resonant modes of the rectangular cavity in both configurations. In the $H_{x}$ configuration, we have used 50 modes in the aperture of the dielectric-loaded cavity and in the T-Junction; 50 accessible modes, 200 basis functions and 600 modes in the series of the integral equation kernel used to characterise the planar junctions between rectangular waveguides. On the other hand, in the $H_{z}$ configuration, we have used 20 modes in the aperture of the dielectric-loaded cavity and in the T-Junction; 20 accessible modes, 150 basis functions and 500 modes in the series of the integral equation kernel for the planar junctions. In Fig. 6.16(a) and Fig. 6.16(b), the results provided by our method are successfully compared to those obtained with Ansoft HFSS for the $H_{x}$ and the $H_{z}$ configurations, respectively.

We can use now the bandstop prototype structure, analogously to the case of the bandpass filter, to design a bandstop filter. For this purpose, we have connected, to a WR-90 waveguide, four dielectric-loaded rectangular cavities. As it is shown in Fig. 6.17, the $H_{x}$ configuration have been used. The filter is symmetric with respect to the axis of the propagation of the WR-90 waveguide. The dielectric supports are assumed to have unitary relative dielectric permittivity with a height $h_{2}=3.25 \mathrm{~mm}$. In the first cavity resonator, the center of the DR is placed at $3.9 \mathrm{~mm}$ from the iris, while in the case of the second resonator is placed at $3.5 \mathrm{~mm}$. The rest of parameters are shown in Table 6.9. In this table, $L_{1}$ is the length from the center of the first iris to the input port, while $L_{2}$ and $L_{3}$, are the length from the center of the second iris to the center of the first one, and the length from the center of the second iris to the center of the third one, respectively.

In order to obtain the electrical response of the bandstop filter, we have employed 200 


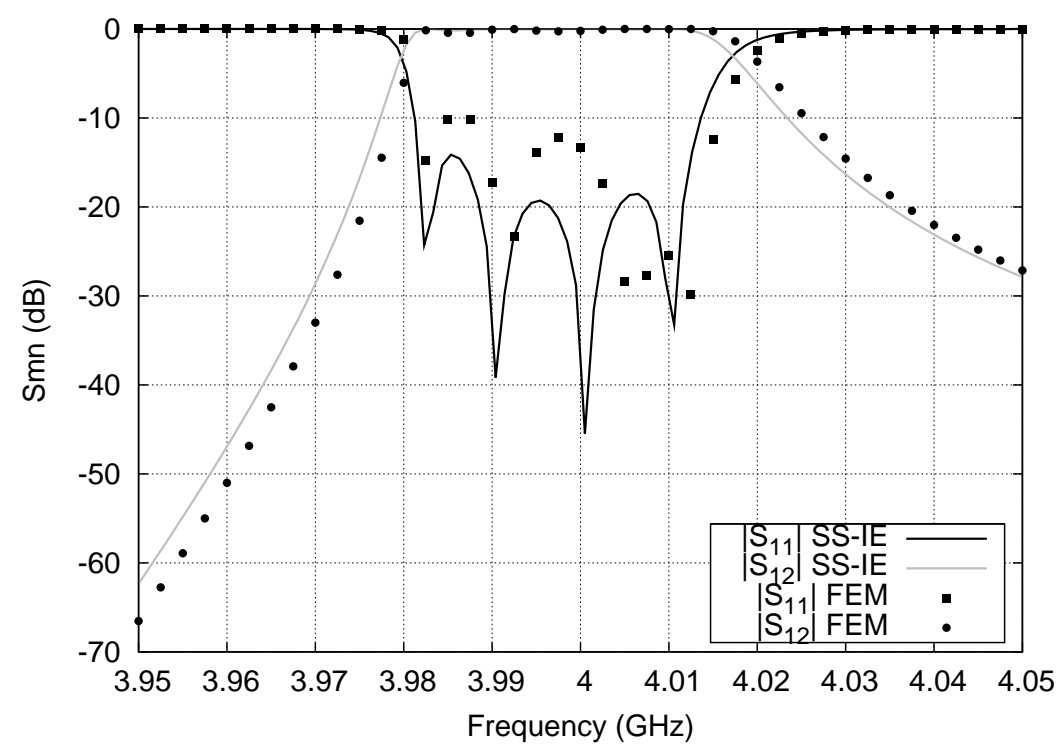

Figure 6.14: Electromagnetic response of the dielectric-loaded rectangular waveguide bandpass filter described in Table 6.7.

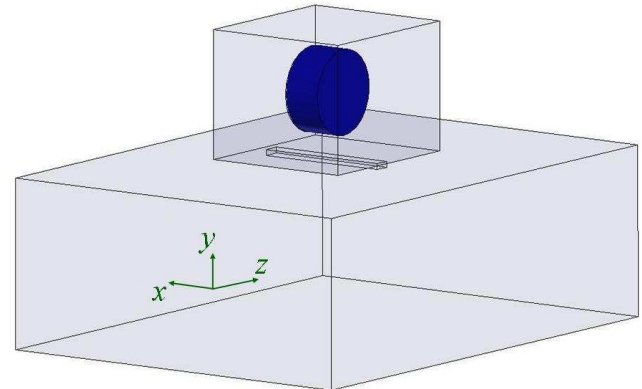

(a) $H_{x}$ configuration

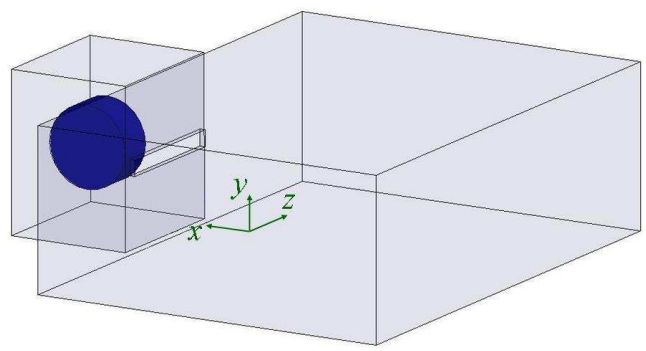

(b) $H_{z}$ configuration

Figure 6.15: Bandstop structures using dielectric resonators in rectangular waveguides.

basis functions, 800 resonant modes of the rectangular cavity 50 modes in the aperture of the dielectric-loaded cavity. To characterise the T-Junction, it has been used 50 modes in the apertures, whereas to analyse the planar junctions between rectangular waveguides 50 accessible modes, 200 basis functions and 700 modes in the series of the integral equation kernel, have been used. To simulate the whole structure, $50 \mathrm{~s}$ have been used in the static part, while $1 \mathrm{~s}$ per frequency point have been employed in the dynamic part using an Intel Core 2 Duo @ $1.83 \mathrm{GHz}$ processor. In Fig. 6.18, the results provided by our method are successfully compared to those obtained with Ansoft HFSS.

Finally, it must be highlighted that the State Space-Integral Equation formulation, due to 


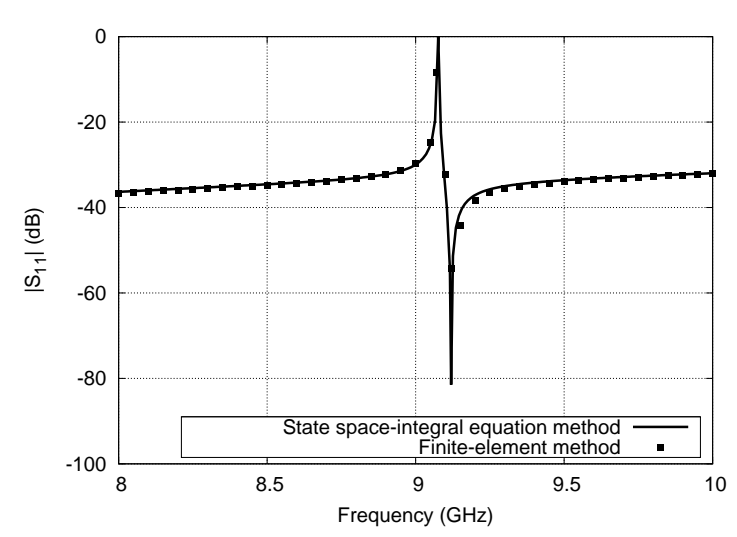

(a) $H_{x}$ configuration

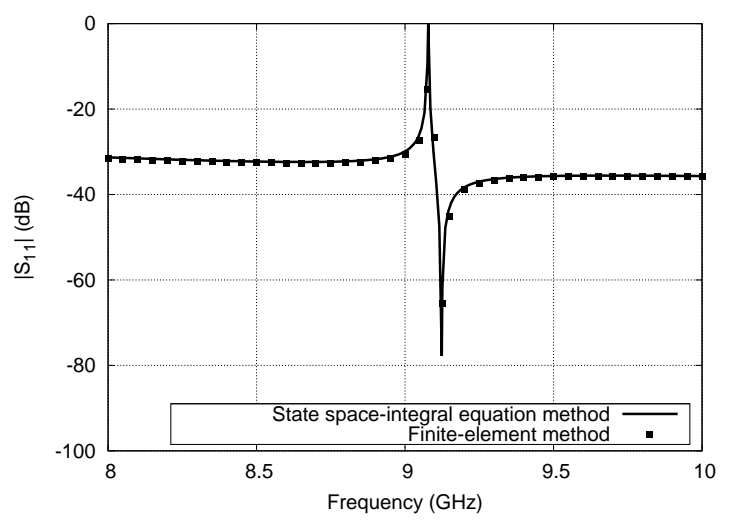

(b) $H_{z}$ configuration

Figure 6.16: Electromagnetic response of the band-stop prototypes. Comparison between the results obtained with the state-space integral-equation method and with Ansoft HFSS.

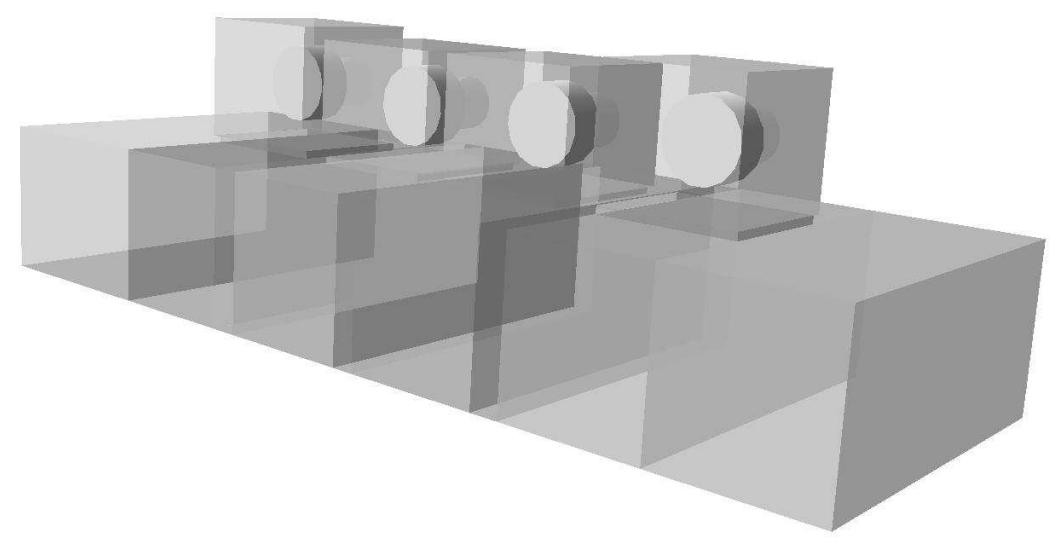

Figure 6.17: Bandstop filter described in Table 6.9.

its numerical efficiency, is very suitable for being used within computer-aided design tools. Most of the total computational effort required by this method is related to the frequency independent calculations needed to build and solve the eigenvalue problem defined by (3.42). Therefore, the optimized design of dielectric-loaded waveguide filters can be very fast, even if a large number of frequency points are used, as long as the optimization parameters are those not affecting the dielectric resonator geometry. For instance, in the filters previously presented, it is possible to control the coupling iris dimensions, as well as the length of the dielectric resonator cavities, to perform a fine adjustment of the S-parameters without changing the dielectric resonator geometry. Since the dielectric resonator geometry is not changed, the solution of the cited eigenvalue problem can be reused, and thus the optimization procedure is extremely efficient. 


\begin{tabular}{||l|ccc||}
\hline \hline Rectangular Cavities & $a(\mathrm{~mm})$ & $b(\mathrm{~mm})$ & $c(\mathrm{~mm})$ \\
& 9.0 & 9.0 & 8.04 \\
& 9.0 & 9.0 & 7.37 \\
\hline Rectangular Irises & $w(\mathrm{~mm})$ & $h(\mathrm{~mm})$ & $t(\mathrm{~mm})$ \\
\hline & 8.88 & 8.0 & 0.5 \\
& 8.03 & 9.0 & 0.5 \\
\hline Lengths between irises & $L_{1}(\mathrm{~mm})$ & $L_{2}(\mathrm{~mm})$ & $L_{3}(\mathrm{~mm})$ \\
\hline & 14.5 & 10.89 & 10.51 \\
\hline Dielectric Resonators & $d(\mathrm{~mm})$ & $h_{1}(\mathrm{~mm})$ & $\varepsilon_{r_{1}}$ \\
\hline & 5.1 & 2.3 & 50 \\
\hline \hline
\end{tabular}

Table 6.9: Dimensions of the bandstop filter based on dielectric-loaded cavities. The dielectric support of the resonators is assumed to have a relative permittivity $\varepsilon_{r_{2}}=1$, and a height of $h_{2}=3.25 \mathrm{~mm}$.

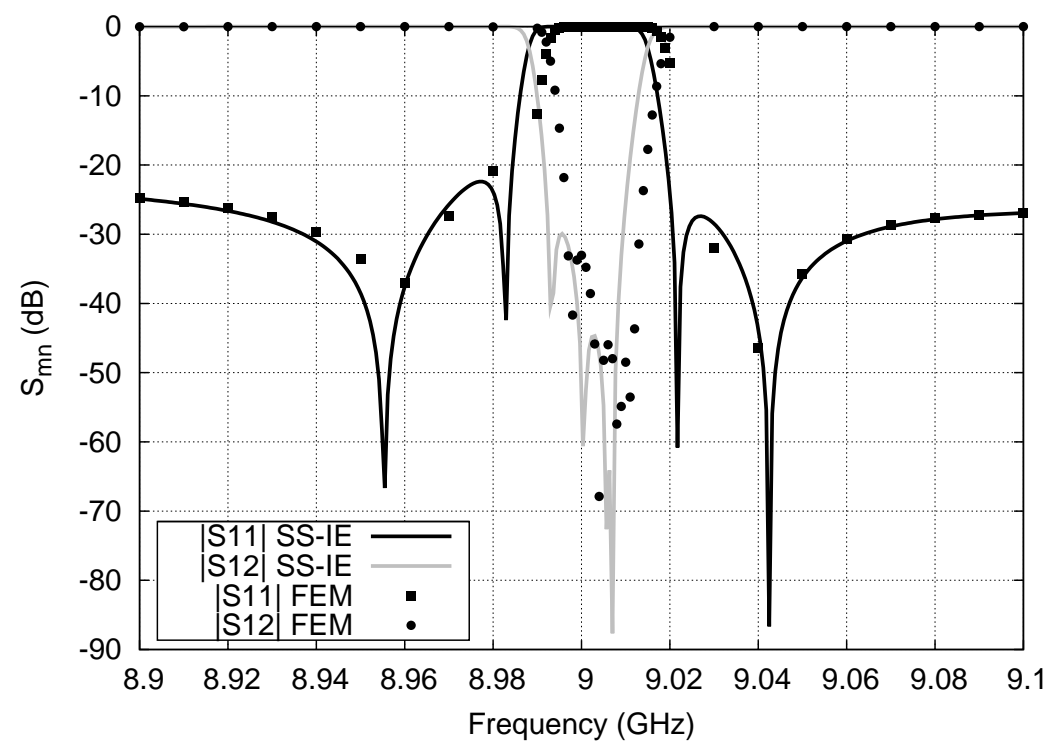

Figure 6.18: Electromagnetic response of the bandstop filter described in Table 6.9. 


\section{Chapter 7}

\section{Conclusions and future work}

In this PhD Thesis, a new State-Space Integral-Equation (SS-IE) formulation in the Laplace variable domain has been proposed for characterizing rectangular cavities loaded with dielectric resonators. This novel technique, based on the BI-RME method, has been successfully implemented to analyse the electromagnetic behaviour of rectangular cavities loaded with cylindrical dielectric resonators. The software module developed in the frame of this Thesis has been integrated in the Computer Aided Engineering (CAE) tool for the full-wave analysis of microwave and millimetre components FEST3D. The use of the resulting tool has permit to analyse and design complex devices, such as stop-band and band-pass filters, based on dielectric loaded resonators in rectangular waveguide technology. The accuracy of the proposed method has been validated through successful comparisons with data obtained from the technical literature and a well known commercial software based on the Finite Element Method (FEM). It has been proved that the implemented method is accurate and computationally efficient, thus making it very suitable for the optimized design of waveguide filters including dielectric resonators.

The SS-IE approach developed in the present Thesis is a Volume Integral Equation formulated in the Laplace variable domain. The linear, homogeneous and isotropic dielectric body is rigorously characterized by means of the electric equivalent polarization charge and current densities defined in the volume of the dielectric object. As shown in Chapter 2, we have expressed the electromagnetic field using the Green's functions of a rectangular cavity instead of using the free-space ones as it is usually done in standard boundary element methods. Thus, by using the Green's functions for the scalar and vector potentials in the Coulomb's gauge, we obtain an hybrid representation of the field in terms of Green's integrals and rapidly converging modal series. Moreover, the Green's functions for a rectangular cavity has been efficiently computed following the Ewald technique. Taking as starting point this hybrid representation of the field and using the Method of Moments (MoM), we can obtain the modal chart and the field distribution of the cavity resonator by solving a real matrix linear eigenvalue problem. Proceeding in this way, we are able to avoid searching the zeros of a matrix determinant as other frequency- or time- domain methods do. In addition, the use of the Green's functions for a rectangular cavity avoids to impose the boundary conditions on the walls of the metallic cavity, and thus reducing the number of unknowns of the problem 
to be solved.

The new formulation has been applied to calculate the modal chart of a rectangular cavity loaded with a cylindrical dielectric resonator placed at an arbitrary position inside the cavity. Throughout the Chapter 4, we have shown how crucial is the selection of an appropriate set of basis functions to expand the polarization vector, not only for the good convergence of the method proposed, but also to transform some of the integrals involved in the computation of the matrices involved in the solution of the problem by means of the MoM. As it is shown, the numerical efficiency of the proposed method depends critically on the computation of these matrices, and specially of those matrices involving the Green's functions. For instance, in this case of the computation of the $\mathbf{L}$ matrix elements, it is crucial not only to transform the singularities of the integrals to regular expressions, but also to reformulate the volumevolume integrals to surface-surface ones. Thus, we only need to perform a 2-D mesh over the surface of the dielectric resonator, instead of performing a 3-D mesh in its volume. These transformations are specially important for the evaluation of the regular part of the $\mathbf{L}$ matrix, since they allow to drastically reduce the required computational effort.

The resonant frequencies of low and high order modes of different rectangular cavities loaded with cylindrical dielectric resonators have been calculated, as well as their electromagnetic field distributions. The influence of geometrical and electric parameters in the computation of the modal chart of the cavity resonator has been studied. The results obtained with the new developed formulation have been successfully compared with the technical literature and with the well-known commercial tool Ansoft High Frequency Structure Simulator (Ansoft HFSS), which is based on the Finite-Element Method. The fact that the algorithm used by HFSS is completely different from the SS-IE formulation presented in this work, makes it a good reference to validate the software developed in this Thesis. On the other hand, the convergence studies performed has shown that the criteria used to order the basis functions used in the MoM can affect the convergence of the method.

Once the code implemented to compute the modal chart of rectangular cavities loaded with cylindrical dielectric resonators was validated, we applied the SS-IE approach to calculate the generalized admittance matrix (GAM) of the considered cavities by opening the lateral access ports. The resonator cavity may be opened through any of its lateral access ports and/or through its top surface. It is important to remark that all the matrices whose elements contain surface integrals over the access ports, involving magnetic Green's functions and magnetic mode vectors for the waveguide, have been analytically solved using the resonant mode expansion of the Green's functions. The efficient analysis of the dielectric resonator loaded cavity by means of the SS-IE approach is a key issue, since the dielectric resonator characterization requires the major part of the computational resources when one analyses more complex structures. As described in Chapter 3, the GAM is obtained as a pole expansion in the domain of the Laplace variable. Hence, with the new formulation we avoid to perform intensive computations at each frequency point like other frequency- and timedomain methods do. Thus, the electromagnetic behaviour of the cavity resonators can be solved in a wide and dense frequency range with a very reduced computational effort. Once the generalized admittance matrix (GAM) of this circuit building block is calculated, it may 
be connected to other circuit elements, in order to analyse different typologies of dielectric resonator filters.

For such a purpose, we have integrated this software module in a Computer Aided Engineering (CAE) tool for the analysis and design of passive microwave and millimetre-waves components: FEST3D (Full-wave Electromagnetic Simulation Tool 3D). The integration into such a general purpose CAE tool permits us to analyze and design different topologies of dielectric resonator filters in waveguide technology. Moreover, it allows us to use all the capabilities of FEST3D when analysing a circuit, such as the 3D visualization of the real component, the use of optimization algorithms, etc. The new tool has been used to design different single-mode bandpass and stopband filters. The designed filters have been used to validate the method developed comparing the results obtained with Ansoft HFSS. As it was expected, the CPU time used to analyse such DR filters is very competitive, being the computations preformed in the frequency loop extremely short.

To conclude, we highlight some of the research activities that may emerge in a future from the work developed in this Thesis. As mentioned in Chapter 3, one of the advantages of $s$-domain solutions is that they may be cast into equivalent electrical circuits, which is crucial in synthesis and design processes. For this reason, one of the next future activities foreseen is to use the developed algorithm in order to find an accurate equivalent electrical circuit that will be used to develop new synthesis tools for dielectric resonator filters. So far, we have applied the formulation developed to analyse rectangular cavities loaded with a cylindrical DR, nevertheless the presence of the dielectric support has not been considered. One possible extension of the work may be to take into account a dielectric support. Moreover, in real applications, the dielectric resonator filters need to be tuned with metallic screws in order to obtain the proper electrical response. In addition, the input/output excitation of such filters is usually achieved by means of coaxial probes. For this reason, it would be of great interest to extend the present formulation to include metal posts in the cavity and to make available the coaxial excitation $[74,120]$. On the other hand, the set of basis functions used in the present work, has been particularized for a cylindrical DR. Despite the fact that the SS-IE formulation presented is based on the solution of a Volume Integral Equation, the election of a suitable set of basis functions has allowed us to reduce the integrals involved to surface integrals. However, if one wants to consider different DR shapes, a new set of basis functions should be considered. And thus, part of the problem should be reformulated. For this reason, in a mid-term future, to find a new SS-IE approach based on a Surface Integral Equation Formulation to analyse cavity resonators with metal and/or dielectric insets may be an optimum objective.

Finally, the scientific publications related to the work performed in this $\mathrm{PhD}$ Thesis are enumerated in Appendix G . 


\section{Appendix A}

\section{Modal analysis of rectangular cavities and waveguides}

\section{A.1 Magnetic modal vectors of a rectangular waveguide}

Let consider a waveguide with rectangular cross section whose dimensions are $l_{x_{\xi}} \times l_{y_{\xi}}$, and whose corresponding transverse coordinates are $\left(x_{\xi}, y_{\xi}\right)$. Then, the magnetic modal vector function related to the $n$-th mode of the waveguide may be expressed as [86]:

$$
\mathbf{h}_{n}^{(\xi)}(\mathbf{r})=\mathcal{N}_{x_{\xi}, n} F_{x_{\xi}, n}\left(x_{\xi}, y_{\xi}\right) \hat{\mathbf{x}}_{\xi}+\mathcal{N}_{y_{\xi}, n} F_{y_{\xi}, n}\left(x_{\xi}, y_{\xi}\right) \hat{\mathbf{y}}_{\xi}
$$

In the last equation, $\mathcal{N}_{x_{\xi}, n}$ and $\mathcal{N}_{y_{\xi}, n}$ are normalization factors whose expression are particularized for the cases $\mathrm{TE}^{z_{\xi}}$ and $\mathrm{TM}^{z_{\xi}{ }^{1}}$ [86]:

$$
\begin{aligned}
\mathcal{N}_{x_{\xi}, n}^{\mathrm{TE}} & =\frac{k_{x_{\xi}, n}}{k_{t, n}^{(\xi)}} \sqrt{\frac{\epsilon_{n_{x_{\xi}}} \epsilon_{n_{y_{\xi}}}}{l_{x_{\xi}} l_{y_{\xi}}}} \\
\mathcal{N}_{y_{\xi}, n}^{\mathrm{TE}} & =\frac{k_{y_{\xi}, n}}{k_{t, n}^{(\xi)}} \sqrt{\frac{\epsilon_{n_{x_{\xi}}} \epsilon_{n_{y_{\xi}}}}{l_{x_{\xi}} l_{y_{\xi}}}} \\
\mathcal{N}_{x_{\xi}, n}^{\mathrm{TM}} & =2 \frac{k_{y_{\xi}, n}}{k_{t, n}^{(\xi)}} \frac{1}{\sqrt{l_{x_{\xi}} l_{y_{\xi}}}} \\
\mathcal{N}_{y_{\xi}, n}^{\mathrm{TM}} & =-2 \frac{k_{x_{\xi}, n}}{k_{t, n}^{(\xi)}} \frac{1}{\sqrt{l_{x_{\xi}} l_{y_{\xi}}}}
\end{aligned}
$$

where $\left(n_{x_{\xi}}, n_{y_{\xi}}\right)$ are the modal indexes of the $n$-th modal solution related to the coordinates $\left(x_{\xi}, y_{\xi}\right)$, respectively; $k_{x_{\xi}, n}=n_{x_{\xi}} \pi / l_{x_{\xi}}$ is the wavenumber related to the coordinate $x_{\xi}$;

\footnotetext{
${ }^{1}$ For the sake of simplicity, hereinafter the superindex $z_{\xi}$ will be omitted to denote the modes $\mathrm{TE}^{z_{\xi}}$ and $\mathrm{TM}^{z_{\xi}}$.
} 
$k_{y_{\xi}, n}=n_{y_{\xi}} \pi / l_{y_{\xi}}$ is the wavenumber related to the coordinate $y_{\xi} ;\left(k_{t, n}^{(\xi)}\right)^{2}=k_{x_{\xi}, n}^{2}+k_{y_{\xi}, n}^{2}$ is the squared wavenumber corresponding to the $n$-th mode of the waveguide; and $\epsilon_{n}$ is the Neumann factor, which is defined as:

$$
\epsilon_{n}=\left\{\begin{array}{l}
1, \text { if } n=0 \\
2, \text { if } n \neq 0
\end{array}\right.
$$

In addition, the functions $F_{x_{\xi}, n}\left(x_{\xi}, y_{\xi}\right)$ and $F_{y_{\xi}, n}\left(x_{\xi}, y_{\xi}\right)$ in the expression (A.1) are defined as follows [86]:

$$
\begin{aligned}
& F_{x_{\xi}, n}\left(x_{\xi}, y_{\xi}\right)=\sin \left(k_{x_{\xi}, n} x_{\xi}\right) \cos \left(k_{y_{\xi}, n} y_{\xi}\right) \\
& F_{y_{\xi}, n}\left(x_{\xi}, y_{\xi}\right)=\cos \left(k_{x_{\xi}, n} x_{\xi}\right) \sin \left(k_{y_{\xi}, n} y_{\xi}\right)
\end{aligned}
$$

Next, we proceed to calculate the divergence of the TE and the TM magnetic modal vectors. In the case of TE modes, the magnetic field may be expressed by means of the following identity:

$$
\mathbf{h}_{n}^{(\xi) \mathrm{TE}}(\mathbf{r})=\hat{\mathbf{z}_{\xi}} \times\left(\hat{\mathbf{z}_{\xi}} \times \frac{1}{k_{t, n}^{(\xi)}} \nabla_{t} \phi_{n}^{(\xi) \mathrm{TE}}(\mathbf{r})\right)
$$

being $z_{\xi}$ the coordinate that defines the propagating direction of the waveguide; $\nabla_{t}$ the transverse gradient operator calculated in the coordinates $\left(x_{\xi}, y_{\xi}\right)$, and $\phi_{n}^{(\xi) \mathrm{TE}}(\mathbf{r})$ the potential function ${ }^{2}$ :

$$
\phi_{n}^{(\xi) \mathrm{TE}}(\mathbf{r})=\sqrt{\frac{\epsilon_{n_{x_{\xi}}} \epsilon_{n_{y_{\xi}}}}{l_{x_{\xi}} l_{y_{\xi}}}} \cos \left(k_{x_{\xi}, n} x_{\xi}\right) \cos \left(k_{y_{\xi}, n} y_{\xi}\right)
$$

Then, we can write ${ }^{3}$ :

$$
\begin{aligned}
\nabla \cdot \mathbf{h}_{n}^{(\xi) \mathrm{TE}}(\mathbf{r})= & \nabla \cdot\left(\hat{\mathbf{z}_{\xi}} \times\left(\hat{\mathbf{z}_{\xi}} \times \frac{1}{k_{t, n}^{(\xi)}} \nabla_{t} \phi_{n}^{(\xi) \mathrm{TE}}(\mathbf{r})\right)\right)=\nabla \cdot\left(\hat{\mathbf{z}_{\xi}} \cdot \frac{1}{k_{t, n}^{(\xi)}} \nabla_{t} \phi_{n}^{(\xi) \mathrm{TE}}(\mathbf{r})\right) \hat{\mathbf{z}_{\xi}} \\
& -\frac{1}{k_{t, n}^{(\xi)}} \nabla \cdot \nabla_{t} \phi_{n}^{(\xi) \mathrm{TE}}(\mathbf{r})=-\frac{1}{k_{t, n}^{(\xi)}} \nabla_{t}^{2} \phi_{n}^{(\xi) \mathrm{TE}}(\mathbf{r})=k_{t, n}^{(\xi)} \phi_{n}^{(\xi) \mathrm{TE}}(\mathbf{r})
\end{aligned}
$$

In the case of TM modes, it can be demonstrated that the divergence of the magnetic modal vector is equal to zero. Since we have:

$$
\mathbf{h}_{n}^{(\xi) \mathrm{TM}}(\mathbf{r})=-\hat{\mathbf{z}_{\xi}} \times \nabla_{t} \phi_{n}^{(\xi) \mathrm{TM}}(\mathbf{r})
$$

being $\phi_{n}^{(\xi) \mathrm{TM}}(\mathbf{r})$ a potential function. Then ${ }^{4}$ :

$$
\begin{aligned}
\nabla \cdot \mathbf{h}_{n}^{(\xi) \mathrm{TM}}(\mathbf{r}) & =-\nabla \cdot\left(\hat{\mathbf{z}_{\xi}} \times \nabla_{t} \phi_{n}^{(\xi) \mathrm{TM}}(\mathbf{r})\right)=\nabla \cdot\left(\nabla_{t} \phi_{n}^{(\xi) \mathrm{TM}}(\mathbf{r}) \times \hat{\mathbf{z}_{\xi}}\right) \\
& =\nabla \cdot\left(\nabla_{t} \times \hat{\mathbf{z}_{\xi}} \phi_{n}^{(\xi) \mathrm{TM}}(\mathbf{r})-\phi_{n}^{(\xi) \mathrm{TM}}(\mathbf{r}) \nabla \times \hat{\mathbf{z}_{\xi}}\right)=0
\end{aligned}
$$

\footnotetext{
${ }^{2}$ The potential function (A.10) satisfies the scalar waves equation.

${ }^{3}$ The following identity has been used: $\mathbf{A} \times(\mathbf{B} \times \mathbf{C})=(\mathbf{A} \cdot \mathbf{C}) \mathbf{B}-(\mathbf{A} \cdot \mathbf{B}) \mathbf{C}$.

${ }^{4}$ The following identity has been used in this step: $\nabla \psi \times \mathbf{A}=\nabla \times(\psi \mathbf{A})-\psi \nabla \times \mathbf{A}$.
} 


\section{A.2 Electric and magnetic solenoidal modal vectors of a rectangular cavity}

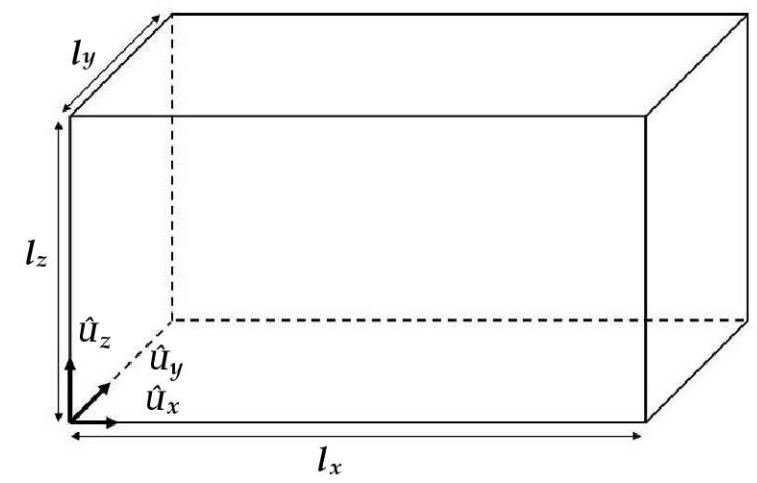

Figure A.1: Rectangular cavity resonator with dimensions $l_{x} \times l_{y} \times l_{z}$.

Let consider a rectangular cavity with dimensions $l_{x} \times l_{y} \times l_{z}$. We assume that $(x, y)$ are the transverse coordinates and $z$ is the axial coordinate (see Figure A.1). In order to obtain the electric and magnetic modal vectors of the rectangular cavity, we should solve equation (2.8b). Nevertheless, the complexity of this vector equation can be relaxed due to the simmetry of the problem, since the cross-section of the cavity is uniform along the $\mathrm{z}$ axis. Thus, the $T E^{z}$ solenoidal modal vectors are related to the eigensolution of the twodimensional equation [62]:

$$
\begin{aligned}
& \nabla^{2} \xi+\lambda^{2} \xi=0 \quad \text { in } \quad S \\
& \frac{\partial \xi}{\partial n}=0 \quad \text { on } \quad \partial S
\end{aligned}
$$

where $\xi$ is a scalar function, $S$ is the surface described by the cavity in the X-Y plane, i. e., the rectangle with dimensions $l_{x} \times l_{y}$, and $\partial S$ its contour.

By means of the eigensolution of this equation, the electric modal vector related to the $i$-th $T E^{z}$ mode can be expressed as:

$$
\vec{E}_{i}^{T E^{z}}=\mathcal{N}_{i}^{T E^{z}} \sin \left(k_{z, i} z\right)\left(\widehat{u}_{z} \times \nabla_{t} \xi_{i_{x}, i_{y}}\right),
$$

and the magnetic modal vector,

$$
\vec{H}_{i}^{T E^{z}}=-\frac{\mathcal{N}_{i}^{T E^{z}}}{k_{i}}\left[k_{z, i} \cos \left(k_{z, i} z\right) \nabla_{t} \xi_{i_{x}, i_{y}}+k_{t, i}^{2} \sin \left(k_{z, i} z\right) \xi_{i_{x}, i_{y}} \widehat{u}_{z}\right]
$$

being $\nabla_{t}$ the transverse gradient operator calculated in the coordinates $(x, y) ; \mathcal{N}_{i}^{T E}$ a normalization factor defined as:

$$
\mathcal{N}_{i}^{T E}=\frac{1}{k_{t, i}} \sqrt{\frac{2 \epsilon_{i_{x}} \epsilon_{i_{y}}}{l_{x} l_{y} l_{z}}}
$$


The scalar function $\xi_{i_{x}, i_{y}}$ is solution of the equation (A.14), and can be written as:

$$
\xi_{i_{x}, i_{y}}=\cos \left(k_{x, i} x\right) \cos \left(k_{y, i} y\right)
$$

By introducing now (A.18) and (A.17) into (A.15) and (A.16), we can the electric and magnetic modal vectors can be rewritten as follows:

$$
\begin{aligned}
\vec{E}_{i}^{T E^{z}}=\frac{1}{k_{t, i}} \sqrt{\frac{2 \epsilon_{i_{x}} \epsilon_{i_{y}}}{l_{x} l_{y} l_{z}}}\left(k_{y, i} \cos \left(k_{x, i} x\right) \sin \left(k_{y, i} y\right) \sin \left(k_{z, i} z\right) \widehat{u}_{x}\right. \\
\left.-k_{x, i} \sin \left(k_{x, i} x\right) \cos \left(k_{y, i} y\right) \sin \left(k_{z, i} z\right) \widehat{u}_{y}\right)
\end{aligned}
$$

and

$$
\begin{aligned}
\vec{H}_{i}^{T E^{z}}=\frac{1}{k_{t, i} k_{i}} \sqrt{\frac{2 \epsilon_{i_{x}} \epsilon_{i_{y}}}{l_{x} l_{y} l_{z}}} & k_{x, i} k_{z, i} \sin \left(k_{x, i} x\right) \cos \left(k_{y, i} y\right) \cos \left(k_{z, i} z\right) \widehat{u}_{x} \\
& +k_{y, i} k_{z, i} \cos \left(k_{x, i} x\right) \sin \left(k_{y, i} y\right) \cos \left(k_{z, i} z\right) \widehat{u}_{y} \\
& \left.-k_{t, i}^{2} \cos \left(k_{x, i} x\right) \cos \left(k_{y, i} y\right) \sin \left(k_{z, i} z\right) \widehat{u}_{z}\right)
\end{aligned}
$$

The $T M^{z}$ solenoidal modal vectors are related to the eigensolution of the two-dimensional equation [62]:

$$
\begin{aligned}
& \nabla^{2} \eta+\lambda^{2} \eta=0 \quad \text { in } \quad S \\
& \eta=0 \quad \text { on } \quad \partial S
\end{aligned}
$$

And thus, the electric modal vector related to the $i$-th $T M^{z}$ mode can be expressed as:

$$
\vec{E}_{i}^{T M^{z}}=\mathcal{N}_{i}^{T M^{z}}\left[k_{z, i} \sin \left(k_{z, i} z\right) \nabla_{t} \eta_{i_{x}, i_{y}}-k_{t, i}^{2} \cos \left(k_{z, i} z\right) \eta_{i_{x}, i_{y}} \widehat{u}_{z}\right]
$$

and the magnetic modal vector,

$$
\vec{H}_{i}^{T M^{z}}=-\mathcal{N}_{i}^{T M^{z}} k_{i} \cos \left(k_{z, i} z\right)\left(\widehat{u}_{z} \times \nabla_{t} \eta_{i_{x}, i_{y}}\right)
$$

where $\mathcal{N}_{i}^{T M}$ is a normailization factor defined as

$$
\mathcal{N}_{i}^{T M}=-\frac{2}{k_{t, i} k_{i}} \sqrt{\frac{\epsilon_{i_{z}}}{l_{x} l_{y} l_{z}}}
$$

The scalar function $\eta_{i_{x}, i_{y}}$ is soltuion of the equation (A.21), and is expressed as:

$$
\eta_{i_{x}, i_{y}}=\sin \left(k_{x, i} x\right) \sin \left(k_{y, i} y\right)
$$


By introducing now (A.24) and (A.25) into (A.22) and (A.23), we can the electric and magnetic modal vectors can be rewritten as follows:

$$
\begin{aligned}
\vec{E}_{i}^{T M^{z}}=-\frac{2}{k_{t, i} k_{i}} \sqrt{\frac{\epsilon_{i_{z}}}{l_{x} l_{y} l_{z}}}( & k_{x, i} k_{z, i} \cos \left(k_{x, i} x\right) \sin \left(k_{y, i} y\right) \sin \left(k_{z, i} z\right) \widehat{u}_{x} \\
& +k_{y, i} k_{z, i} \sin \left(k_{x, i} x\right) \cos \left(k_{y, i} y\right) \sin \left(k_{z, i} z\right) \widehat{u}_{y} \\
& \left.-k_{t, i}^{2} \sin \left(k_{x, i} x\right) \sin \left(k_{y, i} y\right) \cos \left(k_{z, i} z\right) \widehat{u}_{z}\right)
\end{aligned}
$$

and

$$
\begin{aligned}
\vec{H}_{i}^{T M^{z}}=\frac{2}{k_{t, i}} \sqrt{\frac{\epsilon_{i_{z}}}{l_{x} l_{y} l_{z}}}( & k_{y, i} \sin \left(k_{x, i} x\right) \cos \left(k_{y, i} y\right) \cos \left(k_{z, i} z\right) \widehat{u}_{x} \\
& \left.-k_{x, i} \cos \left(k_{x, i} x\right) \sin \left(k_{y, i} y\right) \cos \left(k_{z, i} z\right) \widehat{u}_{y}\right)
\end{aligned}
$$

Please note that in all these expressions, $\left(i_{x}, i_{y}, i_{z}\right)$ are the modal indexes respectively associated to the coordinates $(x, y, z)$ of the $i$-th mode, and $\epsilon_{i}$ is the neumann factor defined in (A.6). Moreover, the following definitions have been considered:

$$
\begin{gathered}
k_{x, i} \equiv \frac{i_{x} \pi}{l_{x}} ; \quad k_{y, i} \equiv \frac{i_{y} \pi}{l_{y}} ; \quad k_{z, i} \equiv \frac{i_{z} \pi}{l_{z}} \\
k_{t, i}^{2} \equiv k_{x, i}^{2}+k_{y, i}^{2}=\lambda ; \quad k_{i}^{2} \equiv k_{t, i}^{2}+k_{z, i}^{2}
\end{gathered}
$$

\section{A.3 Magnetic Green's functions of a rectangular cavity}

Let consider a rectangular cavity with dimensions $l_{x_{\xi}} \times l_{y_{\xi}} \times l_{z_{\xi}}$. We assume that $\left(x_{\xi}, y_{\xi}\right)$ are the transverse coordinates and $z_{\xi}$ is the axial coordinate. The magnetic scalar Green's function can be expressed as follows [62]:

$$
\begin{gathered}
g^{\mathrm{m}}\left(\mathbf{r}, \mathbf{r}^{\prime}\right)=\sum_{\substack{n_{x_{\xi}}, n_{y_{\xi}}, n_{z_{\xi}}=0 \\
n_{x_{\xi}}=n_{y_{\xi}}=n_{z_{\xi}}=0 \text { excluded }}}^{\infty} \frac{\epsilon_{n_{z_{\xi}}}}{\kappa^{2} l_{z_{\xi}}} \phi_{n_{x_{\xi}}, n_{y_{\xi}}}\left(x_{\xi}, y_{\xi}\right) \phi_{n_{x_{\xi}}, n_{y_{\xi}}}\left(x_{\xi}^{\prime}, y_{\xi}^{\prime}\right) \cos \left(k_{z_{\xi}} z_{\xi}\right) \cos \left(k_{z_{\xi}} z_{\xi}^{\prime}\right) \\
\text { (A. }
\end{gathered}
$$


where $\epsilon_{n}$ is the Neumann factor defined in (A.6) and the following definitions have been considered :

$$
\begin{aligned}
\kappa^{2} & =k_{x_{\xi}}^{2}+k_{y_{\xi}}^{2}+k_{z_{\xi}}^{2} \\
k_{\vartheta_{\xi}} & =\frac{n_{\vartheta_{\xi}} \pi}{l_{\vartheta_{\xi}}}, \text { with } \vartheta=(x, y, z) \\
\phi_{n_{x_{\xi}}, n_{y_{\xi}}}\left(x_{\xi}, y_{\xi}\right) & =\sqrt{\frac{\epsilon_{n_{x_{\xi}}} \epsilon_{n_{y_{\xi}}}}{l_{x_{\xi}} l_{y_{\xi}}}} \cos \left(k_{x_{\xi}} x_{\xi}\right) \cos \left(k_{y_{\xi}} y_{\xi}\right)
\end{aligned}
$$

being $\kappa$ the resonant wavenumber of the rectangular cavity. Note that in the expression of the scalar Green's function it appears the potential function used in (A.9) to calculate the TE modes of a rectangular waveguide with dimensions $l_{x_{\xi}} \times l_{y_{\xi}}{ }^{5}$.

Moreover, the static part of the magnetic dyadic Green's function may be expressed as [62]:

$$
\begin{aligned}
\overline{\mathbf{G}}_{0}^{\mathrm{F}}\left(\mathbf{r}, \mathbf{r}^{\prime}\right)= & \sum_{n_{x_{\xi}}, n_{y_{\xi}}=0}^{\infty} \sum_{n_{z_{\xi}}=1}^{\infty} \frac{\mathbf{H}^{\mathrm{TE}}\left(x_{\xi}, y_{\xi}, z_{\xi}\right) \mathbf{H}^{\mathrm{TE}}\left(x_{\xi}^{\prime}, y_{\xi}^{\prime}, z_{\xi}^{\prime}\right)}{\kappa^{2}} \\
& +\sum_{n_{x_{\xi}}, n_{y_{\xi}}=1}^{\infty} \sum_{n_{z_{\xi}}=0}^{\infty} \frac{\mathbf{H}^{\mathrm{TM}}\left(x_{\xi}, y_{\xi}, z_{\xi}\right) \mathbf{H}^{\mathrm{TM}}\left(x_{\xi}^{\prime}, y_{\xi}^{\prime}, z_{\xi}^{\prime}\right)}{\kappa^{2}}
\end{aligned}
$$

where $\mathbf{H}^{\mathrm{TE}}(\mathbf{r})$ and $\mathbf{H}^{\mathrm{TM}}(\mathbf{r})$ are the normalized magnetic modal vectors of the TE and TM modes of a rectangular cavity ${ }^{6}$, respectively, and their expressions are [86]:

$$
\begin{aligned}
\mathbf{H}^{\mathrm{TE}}(\mathbf{r})= & \frac{1}{\kappa} \sqrt{\frac{2}{l_{z_{\xi}}}}\left[k_{z_{\xi}} \cos \left(k_{z_{\xi}} z_{\xi}\right) \mathbf{h}_{n_{x_{\xi}}, n_{y_{\xi}}}^{\mathrm{TE}}(\mathbf{r})\right. \\
& \left.-\sqrt{k_{x_{\xi}}^{2}+k_{y_{\xi}}^{2}} \phi_{n_{x_{\xi}}, n_{y_{\xi}}}\left(x_{\xi}, y_{\xi}\right) \sin \left(k_{z_{\xi}} z_{\xi}\right) \hat{\mathbf{z}}_{\xi}\right] \\
\mathbf{H}^{\mathrm{TM}}(\mathbf{r})= & \sqrt{\frac{\epsilon_{n_{z_{\xi}}}}{l_{z_{\xi}}}} \cos \left(k_{z_{\xi}} z_{\xi}\right) \mathbf{h}_{n_{x_{\xi}}, n_{y_{\xi}}}^{\mathrm{TM}}(\mathbf{r})
\end{aligned}
$$

Finally, the curl of the static part of the magnetic dyadic Green's function may be written

\footnotetext{
${ }^{5}$ Indeed, it can be observed that $\phi\left(x_{\xi}, y_{\xi}\right)=\phi_{n}^{(\xi) \mathrm{TE}}\left(x_{\xi}, y_{\xi}\right)$.

${ }^{6}$ In the first summation term of the expression (A.33) related to the TE modes, the indexes $n_{x_{\xi}}$ and $n_{y_{\xi}}$ can not be equal to zero simultaneously.
} 
as [62]:

$$
\begin{aligned}
\nabla \times \overline{\mathbf{G}}_{0}^{\mathrm{F}}\left(\mathbf{r}, \mathbf{r}^{\prime}\right)= & \sum_{n_{x_{\xi}}, n_{y_{\xi}}=0}^{\infty} \sum_{n_{z_{\xi}}=1}^{\infty} \frac{\mathbf{E}^{\mathrm{TE}}\left(x_{\xi}, y_{\xi}, z_{\xi}\right) \mathbf{H}^{\mathrm{TE}}\left(x_{\xi}^{\prime}, y_{\xi}^{\prime}, z_{\xi}^{\prime}\right)}{\kappa} \\
& +\sum_{n_{x_{\xi}}, n_{y_{\xi}}=1}^{\infty} \sum_{n_{z_{\xi}}=0}^{\infty} \frac{\mathbf{E}^{\mathrm{TM}}\left(x_{\xi}, y_{\xi}, z_{\xi}\right) \mathbf{H}^{\mathrm{TM}}\left(x_{\xi}^{\prime}, y_{\xi}^{\prime}, z_{\xi}^{\prime}\right)}{\kappa}
\end{aligned}
$$

where the normalized electric modal vectors of a rectangular cavity are [86]:

$$
\begin{aligned}
\mathbf{E}^{\mathrm{TE}}(\mathbf{r})= & \sqrt{\frac{2}{l_{z_{\xi}}}}\left(\mathbf{h}_{n_{x_{\xi}}, n_{y_{\xi}}}^{\mathrm{TE}}(\mathbf{r}) \times \hat{\mathbf{z}}_{\xi}\right) \sin \left(k_{z_{\xi}} z_{\xi}\right) \\
\mathbf{E}^{\mathrm{TM}}(\mathbf{r})= & \frac{1}{\kappa} \sqrt{\frac{\epsilon_{n_{z_{\xi}}}}{l_{z_{\xi}}}}\left[\left(\mathbf{h}_{n_{x_{\xi}}, n_{y_{\xi}}}^{\mathrm{TM}}(\mathbf{r}) \times \hat{\mathbf{z}}_{\xi}\right) k_{z_{\xi}} \sin \left(k_{z_{\xi}} z_{\xi}\right)\right. \\
& \left.+\frac{2}{l_{x_{\xi}} l_{y_{\xi}}} \sqrt{k_{x_{\xi}}^{2}+k_{y_{\xi}}^{2}} \sin \left(k_{x_{\xi}} x_{\xi}\right) \sin \left(k_{y_{\xi}} y_{\xi}\right) \cos \left(k_{z_{\xi}} z_{\xi}\right) \hat{\mathbf{z}}_{\xi}\right]
\end{aligned}
$$

Hence, it is remarkable that the electric and magnetic fields of a rectangular cavity with dimensions $l_{x_{\xi}} \times l_{y_{\xi}} \times l_{z_{\xi}}$ have been expressed in terms of the modal vectors $\mathbf{h}_{n}(\mathbf{r})$ of a rectangular waveguide with dimensions $l_{x_{\xi}} \times l_{y_{\xi}}$, defined in (A.9) and (A.12). 


\section{Appendix B}

\section{Integrals involving trigonometric functions}

Let consider the following integral:

$$
I_{\text {trig }} \equiv \int_{0}^{2 \pi} \int_{0}^{2 \pi} F\left(\varphi-\varphi^{\prime}\right)\left\{\begin{array}{c}
\sin (p \varphi) \\
\cos (p \varphi)
\end{array}\right\}\left\{\begin{array}{l}
\sin \left(q \varphi^{\prime}\right) \\
\cos \left(q \varphi^{\prime}\right)
\end{array}\right\} d \varphi d \varphi^{\prime}
$$

where $p$ and $q$ are integers, and $F\left(\varphi-\varphi^{\prime}\right)$ is a function of $\cos \left(\varphi-\varphi^{\prime}\right)$ and/or $\sin \left(\varphi-\varphi^{\prime}\right)$. Since $F\left(\varphi-\varphi^{\prime}\right)$ is a periodic function with period $2 \pi$, then the integral can be transformed into:

$$
I_{\text {trig }} \equiv \int_{\varphi^{\prime}}^{\varphi^{\prime}+2 \pi} \int_{0}^{2 \pi} F\left(\varphi-\varphi^{\prime}\right)\left\{\begin{array}{c}
\sin (p \varphi) \\
\cos (p \varphi)
\end{array}\right\}\left\{\begin{array}{c}
\sin \left(q \varphi^{\prime}\right) \\
\cos \left(q \varphi^{\prime}\right)
\end{array}\right\} d \varphi d \varphi^{\prime}
$$

Next, we can perform the following change of variable: $\alpha=\varphi-\varphi^{\prime} ; d \alpha=d \varphi$. The new boundaries of the integration are:

$$
\varphi=\varphi^{\prime} \Rightarrow \alpha=0 ; \quad \varphi=\varphi^{\prime}+2 \pi \Rightarrow \alpha=2 \pi
$$

And thus, the integral can be rewritten as:

$$
I_{\text {trig }} \equiv \int_{0}^{2 \pi} \int_{0}^{2 \pi} F(\alpha)\left\{\begin{array}{c}
\sin \left(p\left(\alpha+\varphi^{\prime}\right)\right) \\
\cos \left(p\left(\alpha+\varphi^{\prime}\right)\right)
\end{array}\right\}\left\{\begin{array}{c}
\sin \left(q \varphi^{\prime}\right) \\
\cos \left(q \varphi^{\prime}\right)
\end{array}\right\} d \alpha d \varphi^{\prime}
$$

Finally, taking into account the four different sine $(s)$ and/or cosine $(c)$ combinations, and performing the analytical integration over the variable $\varphi^{\prime}$, we obtain:

$$
\begin{aligned}
& I_{\text {trig }}^{(s, s)}=\left(1-\delta_{0 p}\right) \pi \delta_{p q} \int_{0}^{2 \pi} F(\varphi) \cos (p \varphi) d \varphi \\
& I_{\text {trig }}^{(s, c)}=-I_{\text {trig }}^{(c, s)}=\pi \delta_{p q} \int_{0}^{2 \pi} F(\varphi) \sin (p \varphi) d \varphi \\
& I_{\text {trig }}^{(c, c)}=\left(1+\delta_{0 p}\right) \pi \delta_{p q} \int_{0}^{2 \pi} F(\varphi) \cos (p \varphi) d \varphi
\end{aligned}
$$

where $\delta_{p q}$ is the Kronecker delta. 


\section{Appendix C}

\section{Dyadic Analysis}

A dyadic is defined by

$$
\underline{F}=\sum_{j} \vec{F}_{j} \widehat{x}_{j}=\sum_{i} \widehat{x}_{i} \vec{F}_{i}=\sum_{i j} F_{i j} \widehat{x}_{i} \widehat{x}_{j}
$$

The following operations can be defined:

- Scalar products of a Vector and a Dyadic:

$$
\begin{aligned}
& \underline{F} \cdot \vec{a} \equiv \sum_{j} \vec{F}_{j}\left(\widehat{x}_{j} \cdot \vec{a}\right) \\
& \vec{a} \cdot \underline{F} \equiv \sum_{i}\left(\vec{a} \cdot \widehat{x}_{i}\right) \vec{F}_{i}
\end{aligned}
$$

- Vector products of a Vector and a Dyadic:

$$
\begin{aligned}
& \underline{F} \times \vec{a} \equiv \sum_{j} \vec{F}_{j}\left(\widehat{x}_{j} \times \vec{a}\right)=\sum_{i j} F_{i j} \widehat{x}_{i}\left(\widehat{x}_{j} \times \vec{a}\right) \\
& \vec{a} \times \underline{F} \equiv \sum_{i}\left(\vec{a} \times \widehat{x}_{i}\right) \vec{F}_{i}=\sum_{i j} F_{i j}\left(\vec{a} \times \widehat{x}_{i}\right) \widehat{x}_{j}
\end{aligned}
$$

- Divergence and curl of a Dyadic:

$$
\nabla \cdot \underline{F} \equiv \sum_{j}\left(\nabla \cdot \vec{F}_{j}\right) \widehat{x}_{j}
$$




$$
\nabla \times \underline{F} \equiv \sum_{j}\left(\nabla \times \vec{F}_{j}\right) \widehat{x}_{j}
$$

Using these definitions, we can demosntrate some properties of the divergence and the curl of dyadics that will be useful in the development of our formulation. For instance,

$$
\begin{aligned}
\nabla \times(\varphi \underline{F}) & =\sum_{j}\left(\nabla \times\left(\varphi \vec{F}_{j}\right)\right) \widehat{x}_{j}=\sum_{j}\left(\nabla \varphi \times \vec{F}_{j}+\varphi \nabla \times \vec{F}_{j}\right) \widehat{x}_{j} \\
& =\sum_{j}\left(\nabla \varphi \times \vec{F}_{j}\right) \widehat{x}_{j}+\sum_{j}\left(\varphi \nabla \times \vec{F}_{j}\right) \widehat{x}_{j}=\nabla \varphi \times \underline{F}+\varphi \nabla \times \underline{F}
\end{aligned}
$$

where we have used the vector identity $\nabla \times(\varphi \vec{a})=\nabla \varphi \times \vec{a}+\varphi \nabla \times \vec{a}$.

We can also deduce some dyadic integral theorems. Taking as starting point the first vector Green's theorem:

$$
\int_{V}[(\nabla \times \vec{P}) \cdot(\nabla \times \vec{Q})-\vec{P} \nabla \times \nabla \times \vec{Q}] d v=\int_{S_{V}} \hat{n} \cdot(\vec{P} \times \nabla \times \vec{Q}) d s
$$

By considering three different vectors $\vec{Q}_{j}$, so we have three different identities of the same form as (C.7), and by juxtaposing a unit vector $\widehat{x}_{i}$ on the right side and summing, we can write:

$$
\begin{aligned}
\sum_{j}\left(\int_{V}[(\nabla \times \vec{P}) \cdot\right. & \left.\left.\left(\nabla \times \vec{Q}_{j}\right)-\vec{P} \cdot \nabla \times \nabla \times \vec{Q}_{j}\right] d v\right) \widehat{x}_{j} \\
= & \sum_{j}\left(\int_{S_{V}} \widehat{n} \cdot\left(\vec{P} \times \nabla \times \vec{Q}_{j}\right) d s\right) \widehat{x}_{j}
\end{aligned}
$$

Thus, by using the definition of the curl, we demonstrate the so-called first vector-dyadic Green's theorem of Type A:

$$
\int_{V}[(\nabla \times \vec{P}) \cdot(\nabla \times \underline{Q})-\vec{P} \cdot \nabla \times \nabla \times \underline{Q}] d v=\int_{S_{V}} \widehat{n} \cdot(\vec{P} \times \nabla \times \underline{Q}) d s
$$

Using the same procedure but interchanging this time $\vec{P}$ and $\vec{Q}$, we can obtain the so-called first vector-dyadic Green's theorem of Type B:

$$
\int_{V}[(\nabla \times \vec{P}) \cdot(\nabla \times \underline{Q})-(\nabla \times \nabla \times \vec{P}) \cdot \underline{Q}] d v=-\int_{S_{V}} \widehat{n} \cdot(\nabla \times \vec{P} \times \underline{Q}) d s
$$

By substracting (C.9) from (C.10), we obtain the second vector-dyadic Green's theorem:

$$
\begin{aligned}
\int_{V} & {[(\vec{P} \cdot \nabla \times \nabla \times \underline{Q}-(\nabla \times \nabla \times \vec{P}) \cdot \underline{Q}] d v} \\
& =-\int_{S_{V}} \hat{n} \cdot[\vec{P} \times \nabla \times \underline{Q}+(\nabla \times \vec{P}) \times \underline{Q}] d s
\end{aligned}
$$

And using the dyadic identity $\vec{a} \cdot(\vec{b} \times \underline{C})=(\vec{a} \times \vec{b}) \cdot \underline{C}$, we can rewrite

$$
\begin{aligned}
\int_{V} & {[(\vec{P} \cdot \nabla \times \nabla \times \underline{Q}-(\nabla \times \nabla \times \vec{P}) \cdot \underline{Q}] d v} \\
& =-\int_{S_{V}}[(\widehat{n} \times \vec{P}) \cdot \nabla \times \underline{Q}+(\widehat{n} \times \nabla \times \vec{P}) \cdot \underline{Q}] d s
\end{aligned}
$$


Up to this point, the dyadics have been defined using the same coordinate system for both the columns $\left(\widehat{x}_{j}\right)$ and the rows $\left(\widehat{x}_{i}\right)$, but we can define now dyadics with two independent coordinate systems $\{\widehat{x}\}$ and $\left\{\widehat{x}^{\prime}\right\}$. Consider the following dyadic:

$$
\underline{F}\left(\vec{r}, \vec{r}^{\prime}\right)=\sum_{j} \vec{F}_{j} \widehat{x}_{j}^{\prime}=\sum_{i} \widehat{x}_{i} \vec{F}_{i}^{\prime}=\sum_{i j} F_{i j}\left(\vec{r}, \vec{r}^{\prime}\right) \widehat{x}_{i} \widehat{x}_{j}^{\prime}
$$

where $F_{i j}\left(\vec{r}, \vec{r}^{\prime}\right)$ is a scalar function and a vector $\vec{a}^{\prime}$ denotes that this vector $\vec{a}$ has been referenced to the primed coordinate system $\left\{\widehat{x}^{\prime}\right\}$.

In this case, we have to pay attention to the definition of the divergence and the curl, since it may change depending on which coordinate system the operators are refered to. Thus, we have:

$$
\begin{gathered}
\nabla \cdot \underline{F} \equiv \sum_{j}\left(\nabla \cdot \vec{F}_{j}\right) \widehat{x}_{j}^{\prime} \\
\nabla \times \underline{F} \equiv \sum_{j}\left(\nabla \times \vec{F}_{j}\right) \widehat{x}_{j}^{\prime} \\
\nabla^{\prime} \cdot \underline{F} \equiv \sum_{i} \widehat{x}_{i}\left(\nabla^{\prime} \cdot \vec{F}_{i}^{\prime}\right) \\
\nabla^{\prime} \times \underline{F} \equiv \sum_{i} \widehat{x}_{i}\left(\nabla^{\prime} \times \vec{F}_{i}^{\prime}\right)
\end{gathered}
$$

This way, the property expressed by (C.6) in the case of the primed curl is:

$$
\begin{aligned}
& \nabla^{\prime} \times(\varphi \underline{F})=\sum_{i} \widehat{x}_{i}\left(\nabla^{\prime} \times\left(\varphi \vec{F}_{i}^{\prime}\right)\right)=\sum_{i} \widehat{x}_{i}\left(\nabla^{\prime} \varphi \times \vec{F}_{i}^{\prime}+\varphi \nabla^{\prime} \times \vec{F}_{i}^{\prime}\right) \\
& \quad=\sum_{i}-\widehat{x}_{i}\left(\vec{F}_{i}^{\prime} \times \nabla^{\prime} \varphi\right)+\sum_{i} \widehat{x}_{i}\left(\varphi \nabla^{\prime} \times \vec{F}_{i}\right)=-\underline{F} \times \nabla^{\prime} \varphi+\varphi \nabla^{\prime} \times \underline{F}
\end{aligned}
$$

The integral theorems presented above, can be now obtained by juxtaposing in equation (C.7) a unit vector $\widehat{x}_{i}$ on the left side and summing:

$$
\begin{array}{r}
\sum_{i} \widehat{x}_{i}\left(\int_{V}\left[\left(\nabla^{\prime} \times \vec{Q}_{i}\right) \cdot\left(\nabla^{\prime} \times \vec{P}\right)-\left(\nabla^{\prime} \times \nabla^{\prime} \times \vec{Q}_{i}\right) \cdot \vec{P}\right] d v^{\prime}\right) \\
=-\sum_{i} \widehat{x}_{i}\left(\int_{S_{V}}\left(\nabla^{\prime} \times \vec{Q}_{i} \times \vec{P}\right) \cdot \hat{n}^{\prime} d s^{\prime}\right)
\end{array}
$$

This way the first vector-dyadic Green's theorem of Type A can be expressed

$$
\int_{V}\left[\left(\nabla^{\prime} \times \underline{Q}\right) \cdot\left(\nabla^{\prime} \times \vec{P}\right)-\left(\nabla^{\prime} \times \nabla^{\prime} \times \underline{Q}\right) \cdot \vec{P}\right] d v^{\prime}=-\int_{S_{V}}\left(\nabla^{\prime} \times \underline{Q} \times \vec{P}\right) \cdot \widehat{n}^{\prime} d s^{\prime}
$$


And analogously, the first vector-dyadic Green's theorem of Type B:

$$
\int_{V}\left[\left(\nabla^{\prime} \times \underline{Q}\right) \cdot\left(\nabla^{\prime} \times \vec{P}\right)-\underline{Q} \cdot\left(\nabla^{\prime} \times \nabla^{\prime} \times \vec{P}\right)\right] d v^{\prime}=\int_{S_{V}}\left(\underline{Q} \times \nabla^{\prime} \times \vec{P}\right) \cdot \hat{n}^{\prime} d s^{\prime}
$$

And thus, by substracting (C.17) from (C.18), the analog expression for the second vectordyadic Green's theorem is:

$$
\begin{aligned}
\int_{V} & {\left[\left(\nabla^{\prime} \times \nabla^{\prime} \times \underline{Q}\right) \cdot \vec{P}-\underline{Q} \cdot \nabla^{\prime} \times \nabla^{\prime} \times \vec{P}\right] d v^{\prime} } \\
& =\int_{S_{V}}\left[\left(\nabla^{\prime} \times \underline{Q}\right) \times \vec{P}+\underline{Q} \times \nabla^{\prime} \times \vec{P}\right] \cdot \widehat{n}^{\prime} d s
\end{aligned}
$$

Finally, using the dyadic indentity $(\underline{C} \times \vec{a}) \cdot \vec{b}=\underline{C} \cdot(\vec{a} \times \vec{b})$, we can rewrite:

$$
\begin{aligned}
\int_{V} & {\left[\left(\nabla^{\prime} \times \nabla^{\prime} \times \underline{Q}\right) \cdot \vec{P}-\underline{Q} \cdot \nabla^{\prime} \times \nabla^{\prime} \times \vec{P}\right] d v^{\prime} } \\
& =-\int_{S_{V}}\left[\left(\nabla^{\prime} \times \underline{Q}\right) \cdot\left(\hat{n}^{\prime} \times \vec{P}\right)+\underline{Q} \cdot\left(\hat{n}^{\prime} \times \nabla^{\prime} \times \vec{P}\right)\right] d s^{\prime}
\end{aligned}
$$




\section{Appendix D}

\section{Integrals involved in the computation of Matrix C}

In Chapter 4, the computation of the matrices needed to solve the electromagnetic behaviour of a rectangular cavity loaded with a cylindrical DR using the MoM, have been detailed. The entries of some of these matrices are integrals that involve the Green's functions of the rectangular cavity. Consequently, to perform these integrations, special attention must be paid to the singularities of the Green's functions. In this Appendix, we detail some of the procedures that have been followed in order to compute the matrix $\mathbf{C}$.

\section{D.1 Integral involved in the computation of the matrix $\tilde{C}^{(1,1)}$}

To compute the entries of the matrix $\tilde{C}^{(1,1)}$, we need to calculate integrals of this kind:

$$
I=\int_{S} \int_{S} \frac{f(\vec{r}) g\left(\vec{r}^{\prime}\right)}{R} d s d s^{\prime}
$$

where $f(\vec{r})$ and $g\left(\vec{r}^{\prime}\right)$ are scalar functions; $R=\left|\vec{r}-\vec{r}^{\prime}\right| ; S$ is a planar surface, which is the domain of integration. This integrand is singular when $\vec{r}=\vec{r}^{\prime}(R=0)$. To avoid this singularity, we make use of one of the results obtained from the work of P. Arcioni et al. [106], that permits to express the term $1 / R$ as:

$$
\frac{1}{R}=-\nabla_{s} \cdot \nabla_{s}^{\prime} R
$$

where $\nabla_{s}$ is the surface nabla operator. It is remarkable that this result is only applicable when $\vec{r}$ and $\vec{r}^{\prime}$ are on a coplanar surface.

Thus, introducing (D.2) into (D.1), the integral can be rewritten as:

$$
I=-\int_{S} \int_{S} f(\vec{r}) g\left(\vec{r}^{\prime}\right) \nabla_{s} \cdot \nabla_{s}^{\prime} R d s d s^{\prime}=-\int_{S} g\left(\vec{r}^{\prime}\right)\left[\int_{S} f(\vec{r}) \nabla_{s} \cdot \nabla_{s}^{\prime} R d s\right] d s^{\prime}
$$


We can further develop this last expression. For this purpose, we can use the following vector identity on a surface $[85]^{1}$

$$
\int_{S} \vec{P} \cdot \nabla_{s} \psi d s=\int_{C} \psi \vec{P} \cdot \widehat{u}_{m} d l-\int_{S} \psi \nabla_{s} \cdot \vec{P} d s
$$

where $\vec{P}$ and $\psi$ are a vector and a scalar function defined on the surface $S$ with contour $C$, and $\widehat{u}_{m}$ is the unit vector that is on the tangent plane and perpendicular to curve $C$.

By using (D.5), we can transform the integration over $\vec{r}$ in (D.3),

$$
\int_{S} f(\vec{r}) \nabla_{s} \cdot \nabla_{s}^{\prime} R d s=\int_{C} f(\vec{r})\left(\nabla_{s}^{\prime} R\right) \cdot \widehat{u}_{m} d l-\int_{S}\left(\nabla_{s}^{\prime} R\right) \cdot \nabla_{s} f(\vec{r}) d s
$$

and thus, the new expression of the integral is:

$$
I=-\int_{S} \int_{C} g\left(\vec{r}^{\prime}\right) f(\vec{r})\left(\nabla_{s}^{\prime} R\right) \cdot \widehat{u}_{m} d l d s^{\prime}+\int_{S} \int_{S} g\left(\vec{r}^{\prime}\right)\left(\nabla_{s}^{\prime} R\right) \cdot \nabla_{s} f(\vec{r}) d s d s^{\prime}
$$

Next, following an analogous procedure to transform the integration over $\vec{r}^{\prime}$ in the two terms of expression (D.7), we have:

$$
\begin{gathered}
\int_{S} \int_{C} g\left(\vec{r}^{\prime}\right) f(\vec{r})\left(\nabla_{s}^{\prime} R\right) \cdot \widehat{u}_{m} d l d s^{\prime}= \\
\int_{C} \int_{C} R f(\vec{r}) g\left(\vec{r}^{\prime}\right) \widehat{u}_{m} \cdot \widehat{u}_{m}^{\prime} d l d l^{\prime}-\int_{S} \int_{C} f(\vec{r}) R \nabla_{s}^{\prime} \cdot\left(g\left(\vec{r}^{\prime}\right) \widehat{u}_{m}\right) d s^{\prime} d l \\
\int_{S} \int_{S} g\left(\vec{r}^{\prime}\right)\left(\nabla_{s}^{\prime} R\right) \cdot \nabla_{s} f(\vec{r}) d s d s^{\prime}= \\
\int_{S} \int_{C} R g\left(\vec{r}^{\prime}\right)\left(\nabla_{s} f(\vec{r})\right) \cdot \widehat{u}_{m}^{\prime} d l^{\prime} d s-\int_{S} \int_{S} R\left(\nabla_{s} f(\vec{r})\right) \cdot\left(\nabla_{s}^{\prime} g\left(\vec{r}^{\prime}\right)\right) d s d s^{\prime}
\end{gathered}
$$

Finally, by introducing (D.8) and (D.9) into (D.7), we obtain:

$$
\begin{aligned}
I & =\int_{S} \int_{C} f(\vec{r}) R \nabla_{s}^{\prime} \cdot\left(g\left(\vec{r}^{\prime}\right) \widehat{u}_{m}\right) d s^{\prime} d l+\int_{S} \int_{C} R g\left(\vec{r}^{\prime}\right)\left(\nabla_{s} f(\vec{r})\right) \cdot \widehat{u}_{m}^{\prime} d l^{\prime} d s \\
& -\int_{S} \int_{S} R\left(\nabla_{s} f(\vec{r})\right) \cdot\left(\nabla_{s}^{\prime} g\left(\vec{r}^{\prime}\right)\right) d s d s^{\prime}-\int_{C} \int_{C} R f(\vec{r}) g\left(\vec{r}^{\prime}\right) \widehat{u}_{m} \cdot \widehat{u}_{m}^{\prime} d l d l^{\prime}
\end{aligned}
$$

${ }^{1}$ The general expression, for any kind of surface, is:

$$
\int_{S} \vec{P} \cdot \nabla_{s} \psi d s=\int_{C} \psi \vec{P} \cdot \widehat{u}_{m} d l-\int_{S}\left(\psi \nabla_{s} \cdot \vec{P}+J \psi \widehat{u}_{n} \cdot \vec{P}\right) d s
$$

where $J$ is the first curvature of the surface, and $\widehat{u}_{n}$ is its unit normal vector. In our case, $J=0$. 


\section{D.2 Integral involved in the computation of the matrix $\tilde{C}^{(3,3)}$}

As it has been shown in Chapter 4, the computation of the $\tilde{C}^{(3,3)}$ matrix requires the evaluation of the following singular integral:

$$
I=\int_{0}^{h} \int_{0}^{h} \int_{0}^{2 \pi} \frac{\sin \left(\beta_{l}^{h} z\right) \sin \left(\beta_{t}^{h} z^{\prime}\right) \cos (p \varphi)}{\sqrt{d^{2}(1-\cos \varphi) / 2+\left(z-z^{\prime}\right)^{2}}} d z d z^{\prime} d \varphi
$$

Since the integrand has an odd dependence with respect to the variable $\varphi$, we can easily change the limits of the integration over this variable from $[0,2 \pi[$ to $[0, \pi[$ by multiplying the integral by a factor 2 . In addition, to isolate the singularity, we may perform the change of variable $t=z-z^{\prime}$, obtaining

$$
I=2 \int_{0}^{\pi} d \varphi \int_{0}^{h} d z^{\prime} \int_{-z^{\prime}}^{h-z^{\prime}} d t \frac{g\left(t, z^{\prime}, \varphi\right)}{\sqrt{d^{2} \sin ^{2} \frac{\varphi}{2}+t^{2}}}
$$

where we have taken into account the identity $1-\cos \varphi=2 \sin ^{2} \frac{\varphi}{2}$, and the regular function $g\left(t, z^{\prime}, \varphi\right)$ has been defined as:

$$
g\left(t, z^{\prime}, \varphi\right) \equiv \sin \left(\beta_{l}^{h}\left(t+z^{\prime}\right)\right) \sin \left(\beta_{t}^{h} z^{\prime}\right) \cos (p \varphi)
$$

Next, by adding and subtracting the term $\left(\left(d \frac{\varphi}{2}\right)^{2}+t^{2}\right)^{-1 / 2}$, we can rewrite the integral as:

$$
I=2\left(I_{1}+I_{2}\right)
$$

where the following integrals have been defined:

$$
\begin{aligned}
I_{1} & \equiv \int_{0}^{\pi} d \varphi \int_{0}^{h} d z^{\prime} \int_{-z^{\prime}}^{h-z^{\prime}} d t g\left(t, z^{\prime}, \varphi\right)\left(\frac{1}{\sqrt{d^{2} \sin ^{2} \frac{\varphi}{2}+t^{2}}}-\frac{1}{\sqrt{\left(d \frac{\varphi}{2}\right)^{2}+t^{2}}}\right) \\
I_{2} & \equiv \int_{0}^{\pi} d \varphi \int_{0}^{h} d z^{\prime} \int_{-z^{\prime}}^{h-z^{\prime}} d t g\left(t, z^{\prime}, \varphi\right) \frac{1}{\sqrt{\left(d^{\left.\frac{\varphi}{2}\right)^{2}+t^{2}}\right.}}
\end{aligned}
$$

Firstly, we proceed to evaluate the integral $I_{1}$. It can be observed in (D.15) that the integrand of this expression is made up of the multiplication between a function, which is regular and bounded in the domain of integration, and a singular term that may be rewritten as follows:

$$
\begin{aligned}
& \frac{1}{\sqrt{d^{2} \sin ^{2} \frac{\varphi}{2}+t^{2}}}-\frac{1}{\sqrt{\left(d \frac{\varphi}{2}\right)^{2}+t^{2}}}=\frac{\sqrt{\left(d \frac{\varphi}{2}\right)^{2}+t^{2}}-\sqrt{d^{2} \sin ^{2} \frac{\varphi}{2}+t^{2}}}{\sqrt{d^{2} \sin ^{2} \frac{\varphi}{2}+t^{2}} \sqrt{\left(d \frac{\varphi}{2}\right)^{2}+t^{2}}}= \\
& \frac{\left(d \frac{\varphi}{2}\right)^{2}-\left(d \sin \frac{\varphi}{2}\right)^{2}}{\sqrt{d^{2} \sin ^{2} \frac{\varphi}{2}+t^{2}} \sqrt{\left(d \frac{\varphi}{2}\right)^{2}+t^{2}}\left(\sqrt{d^{2} \sin ^{2} \frac{\varphi}{2}+t^{2}}+\sqrt{\left(d \frac{\varphi}{2}\right)^{2}+t^{2}}\right)}= \\
& \frac{d \frac{\varphi}{2}+d \sin \frac{\varphi}{2}}{\sqrt{d^{2} \sin ^{2} \frac{\varphi}{2}+t^{2}}+\sqrt{\left(d \frac{\varphi}{2}\right)^{2}+t^{2}}} \frac{d \frac{\varphi}{2}-d \sin \frac{\varphi}{2}}{\sqrt{d^{2} \sin ^{2} \frac{\varphi}{2}+t^{2}} \sqrt{\left(d \frac{\varphi}{2}\right)^{2}+t^{2}}}
\end{aligned}
$$


Let see now if this expression is bounded in the integration domain. Taking into account that $\varphi \in[0, \pi[$, it is verified that

$$
\begin{aligned}
& \sqrt{\left(d \frac{\varphi}{2}\right)^{2}+t^{2}} \geq \sqrt{\left(d \frac{\varphi}{2}\right)^{2}}=d \frac{\varphi}{2} \\
& \sqrt{\left(d \sin \frac{\varphi}{2}\right)^{2}+t^{2}} \geq \sqrt{\left(d \sin \frac{\varphi}{2}\right)^{2}}=d \sin \frac{\varphi}{2}
\end{aligned}
$$

and then,

$$
0 \leq \frac{d \frac{\varphi}{2}+d \sin \frac{\varphi}{2}}{\sqrt{d^{2} \sin ^{2} \frac{\varphi}{2}+t^{2}}+\sqrt{\left(d \frac{\varphi}{2}\right)^{2}+t^{2}}} \leq 1
$$

On the other hand, we also have

$$
\frac{d \frac{\varphi}{2}-d \sin \frac{\varphi}{2}}{\sqrt{d^{2} \sin ^{2} \frac{\varphi}{2}+t^{2}} \sqrt{\left(d \frac{\varphi}{2}\right)^{2}+t^{2}}} \leq \frac{\left(\frac{\varphi}{2}-\sin \frac{\varphi}{2}\right)}{\left(d \sin \frac{\varphi}{2}\right)\left(\frac{\varphi}{2}\right)} \leq \frac{\pi\left(\frac{\varphi}{2}-\sin \frac{\varphi}{2}\right)}{d \frac{\varphi^{2}}{2}}
$$

In this last inequality we have taken into account that $(2 / \pi) u \leq \sin u$ when $u \in[0, \pi / 2[$. Furthermore, it is easily verified using the Taylor series that

$$
x-\sin x \simeq \frac{x^{3}}{3 !}-\frac{x^{5}}{5 !}+\frac{x^{7}}{7 !} \cdots \Rightarrow x-\sin x \leq \frac{x^{3}}{3 !}, \quad x \in[0, \pi[
$$

And thus, we can rewrite inequality (D.19) as:

$$
\frac{d \frac{\varphi}{2}-d \sin \frac{\varphi}{2}}{\sqrt{d^{2} \sin ^{2} \frac{\varphi}{2}+t^{2}} \sqrt{\left(d \frac{\varphi}{2}\right)^{2}+t^{2}}} \leq \frac{\pi\left(\frac{\varphi}{2}-\sin \frac{\varphi}{2}\right)}{d \frac{\varphi^{2}}{2}} \leq \frac{\pi\left(\frac{\varphi}{2}\right)^{3} / 3 !}{d \frac{\varphi^{2}}{2}}=\frac{\varphi \pi}{24 d}, \quad \varphi \in[0, \pi[
$$

From expressions (D.18) and (D.20) it is deduced that the singular term of the $I_{1}$ integrand (eq. (D.17)) is bounded. Moreover, we can calculate the limit:

$$
\begin{gathered}
\lim _{\varphi \rightarrow 0^{+}} \frac{1}{\sqrt{d^{2} \sin ^{2} \frac{\varphi}{2}+t^{2}}}-\left.\frac{1}{\sqrt{\left(d \frac{\varphi}{2}\right)^{2}+t^{2}}}\right|_{t \neq 0}=\lim _{\varphi \rightarrow 0^{+}} \frac{1}{\sqrt{t^{2}}}-\frac{1}{\sqrt{t^{2}}} \\
\lim _{\varphi \rightarrow 0^{+}} \frac{1}{\sqrt{d^{2} \sin ^{2} \frac{\varphi}{2}+t^{2}}}-\left.\frac{1}{\sqrt{\left(d \frac{\varphi}{2}\right)^{2}+t^{2}}}\right|_{t=0}= \\
\lim _{\varphi \rightarrow 0^{+}} \frac{1}{\sqrt{\left(d \sin \frac{\varphi}{2}\right)^{2}}}-\frac{1}{\sqrt{\left(d \frac{\varphi}{2}\right)^{2}}}=\lim _{\varphi \rightarrow 0^{+}} \frac{1}{\frac{d \varphi}{2}}-\frac{1}{d \frac{\varphi}{2}}=0
\end{gathered}
$$

Therefore, the integral $I_{1}$ is Riemann integrable, since its integrand is continuous and bounded in the integration domain. Thus, we may compute it numerically taking into account that the integrand is zero when $\varphi=0$.

We proceed now to calculate the integral $I_{2}$, whose integrand is singular when $\varphi=t=0$. Firstly, we perform a change of variable:

$$
u=d \frac{\varphi}{2} ; \quad d u=\frac{d}{2} d \varphi ; \quad u \in[0, d \pi / 2[
$$


Consequently, the integral can be written as:

$$
\begin{aligned}
I_{2} & =\int_{0}^{\pi} d \varphi \int_{0}^{h} d z^{\prime} \int_{-z^{\prime}}^{h-z^{\prime}} d t g\left(t, z^{\prime}, \varphi\right) \frac{1}{\sqrt{\left(d \frac{\varphi}{2}\right)^{2}+t^{2}}} \\
& =\frac{2}{d} \int_{0}^{d \pi / 2} d \varphi \int_{0}^{h} d z^{\prime} \int_{-z^{\prime}}^{h-z^{\prime}} d t g\left(t, z^{\prime}, u\right) \frac{1}{\sqrt{u^{2}+t^{2}}}
\end{aligned}
$$

Next we perform a second change of variable:

$$
\left.\begin{array}{l}
u=\rho \cos \theta \\
t=\rho \sin \theta
\end{array}\right\} \Rightarrow \quad J(\rho, \theta)=\left|\begin{array}{ll}
\frac{\partial u}{\partial \rho} & \frac{\partial u}{\partial \theta} \\
\frac{\partial t}{\partial \rho} & \frac{\partial t}{\partial \theta}
\end{array}\right|=\left|\begin{array}{cc}
\cos \theta & -\rho \sin \theta \\
\sin \theta & \rho \cos \theta
\end{array}\right|=\rho
$$

where $\rho$ and $\theta$ are the new variables, and $J(\rho, \theta)$ is the Jacobian determinant associated to the change of variables.

Thus, the integral can be rewritten as follows,

$$
I_{2}=\frac{2}{d} \int_{0}^{h} d z^{\prime} \int_{S_{\rho, \theta}} \rho d \rho d \theta \frac{g\left(\rho, z^{\prime}, \theta\right)}{\sqrt{(\rho \cos \theta)^{2}+(\rho \sin \theta)^{2}}}=\frac{2}{d} \int_{0}^{h} d z^{\prime} \int_{S_{\rho, \theta}} d \rho d \theta g\left(\rho, z^{\prime}, \theta\right)
$$

and introducing the expression of the function $g$, we have:

$$
I_{2}=\frac{2}{d} \int_{0}^{h} d z^{\prime} \int_{S_{\rho, \theta}} d \rho d \theta \sin \left(\beta_{l}^{h}\left(\rho \sin \theta+z^{\prime}\right)\right) \sin \left(\beta_{t}^{h} z^{\prime}\right) \cos \left(\frac{2 p}{d} \rho \cos \theta\right)
$$

Let remark that the singularity has been canceled due to the introduction of the Jacobian. In this new expression, $S_{\rho, \theta}$ is the domain of integration associated to the variables $\rho$ and $\theta$. It is important to notice that the integrand is regular, and thus we can compute the integral numerically. Taking into account that the boundaries of integration are $u \in\left[0, \frac{d \pi}{2}[\right.$ and $t \in\left[-z^{\prime}, h-z^{\prime}\right]$, we consider three different regions of integration in $S_{\rho, \theta}$. For this purpose, we define the following parameters (see Fig. D.1):

$$
\begin{array}{ll}
\tan \theta_{1}=\frac{-2 z^{\prime}}{\pi d} & \text { with } \quad \theta_{1} \leq 0, z^{\prime} \in[0, h] \\
\tan \theta_{2}=\frac{2\left(h-z^{\prime}\right)}{\pi d} & \text { with } \quad \theta_{2} \geq 0, h-z^{\prime} \in[h, 0]
\end{array}
$$

And finally, the three regions of integration are:

- Region 1: $\theta \in\left[-\pi / 2, \theta_{1}\left[, \quad \sin \theta=\frac{-z^{\prime}}{\rho_{\max 1}} \rightarrow \rho_{\max 1}=\frac{-z^{\prime}}{\sin \theta} \rightarrow \rho \in\left[0, \frac{-z^{\prime}}{\sin \theta}\right]\right.\right.$

- Region 2: $\theta \in\left[\theta_{1}, \theta_{2}\left[, \quad \cos \theta=\frac{\pi d}{2 \rho_{\max 2}} \rightarrow \rho_{\max 2}=\frac{\pi d}{2 \cos \theta} \rightarrow \rho \in\left[0, \frac{\pi d}{2 \cos \theta}\right]\right.\right.$

- Region 3: $\theta \in\left[\theta_{2}, \pi / 2\right], \quad \sin \theta=\frac{h-z^{\prime}}{\rho_{\max 3}} \rightarrow \rho_{\max 3}=\frac{h-z^{\prime}}{\sin \theta} \rightarrow \rho \in\left[0, \frac{h-z^{\prime}}{\sin \theta}\right]$

where $\rho_{\text {maxi }}$ is the maximum value that $\rho$ can takes for a given value of $\theta$ in the $i$-region. 


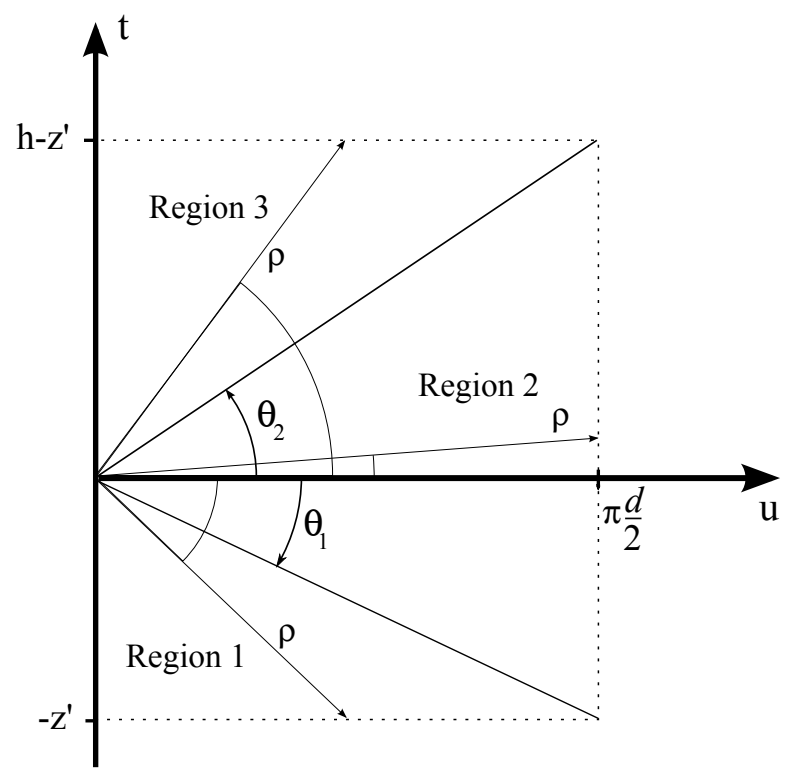

Figure D.1: Graphical representation of the domain of integration $S_{\rho, \theta}$ associated to the variables $\rho$ and $\theta$. The regions in which $S_{\rho, \theta}$ is divided to calculate numerically the integral are also represented.

\section{D.3 Integral involved in the computation of the matrix $\tilde{C}^{(1,3)}$}

In the section 4.3 , it has been shown that in order to calculate the entries of the matrix $\tilde{C}^{(1,3)}$, the following integral must be computed:

$$
\tilde{I}_{p q, t}^{(1,3)} \equiv \int_{0}^{\frac{d}{2}} d \rho \int_{0}^{h} d z^{\prime} \int_{0}^{2 \pi} d \varphi \frac{\rho J_{p}\left(\Theta_{p q} \rho\right) \sin \left(\beta_{t}^{h} z^{\prime}\right) \cos (p \varphi)}{\sqrt{\rho^{2}+d^{2} / 4-\rho d \cos (\varphi)+z^{\prime 2}}}
$$

where $J_{p}(X)$ is the Bessel function of the first kind of order $p ; \Theta_{p q}=\frac{2 \chi_{p q}}{d}$ and $\beta_{t}^{h}=\frac{t \pi}{h}$, being $\chi_{p q}$ the q-th non-zero root of $J_{p}(X)=0 ; \rho \in[0, d / 2] ; \varphi \in\left[0,2 \pi\left[\right.\right.$; and $z^{\prime} \in[0, h]$. It is easily observed that when $\rho=d / 2, \varphi=0$ and $z^{\prime}=0$, the integrand has an indeterminacy of the kind $0 / 0$. Due to this fact, we need to evaluate this indeterminacy. In this section, we demonstrate that this limit exists:

$$
\lim _{\left\{\rho, \varphi, z^{\prime}\right\} \rightarrow\{d / 2,0,0\}} \frac{\rho J_{p}\left(\Theta_{p q} \rho\right) \sin \left(\beta_{t}^{h} z^{\prime}\right) \cos (p \varphi)}{\sqrt{\rho^{2}+d^{2} / 4-\rho d \cos (\varphi)+z^{\prime 2}}}
$$

Firstly, we can observe that in the vicinity of $\varphi=0$, since $\cos \varphi>0$, the following inequality holds:

$$
\rho^{2}+d^{2} / 4-\rho d \cos (\varphi) \geq \rho^{2}+d^{2} / 4-\rho d
$$

Furthermore, we define the function:

$$
f(\rho) \equiv \rho^{2}+d^{2} / 4-\rho d
$$


This function is monotonically decreasing in the range of interest, i.e. $\rho \in[0, d / 2]$, with $f(0)=d^{2} / 4$ and $f(d / 2)=0$. Thus, we have

$$
\rho^{2}+d^{2} / 4-\rho d \geq 0
$$

Therefore, the following inequalities are also satisfied:

$$
\begin{gathered}
\rho^{2}+d^{2} / 4-\rho d \cos (\varphi)+\left(z^{\prime}\right)^{2} \geq\left(z^{\prime}\right)^{2} \\
\sqrt{\rho^{2}+d^{2} / 4-\rho d \cos (\varphi)+z^{\prime 2}} \geq\left|z^{\prime}\right|
\end{gathered}
$$

Finally, taking into account this last expression and $|\sin x| \leq|x|$, we can write:

$$
\left|\frac{\rho J_{p}\left(\Theta_{p q} \rho\right) \sin \left(\beta_{t}^{h} z^{\prime}\right) \cos (p \varphi)}{\sqrt{\rho^{2}+d^{2} / 4-\rho d \cos (\varphi)+z^{\prime 2}}}\right| \leq \frac{\left|\rho J_{p}\left(\Theta_{p q} \rho\right)\right|\left|\beta_{t}^{h} z^{\prime}\right|}{\left|z^{\prime}\right|}=\left|\rho J_{p}\left(\Theta_{p q} \rho\right)\right|\left|\beta_{t}^{h}\right|
$$

And thus, since

$$
\lim _{\rho \rightarrow d / 2}\left|\rho J_{p}\left(\Theta_{p q} \rho\right)\right|\left|\beta_{t}^{h}\right|=0,
$$

then it is demonstrated that the limit of the integrand exists and it is:

$$
\lim _{\left\{\rho, \varphi, z^{\prime}\right\} \rightarrow\{d / 2,0,0\}} \frac{\rho J_{p}\left(\Theta_{p q} \rho\right) \sin \left(\beta_{t}^{h} z^{\prime}\right) \cos (p \varphi)}{\sqrt{\rho^{2}+d^{2} / 4-\rho d \cos (\varphi)+z^{\prime 2}}}=0
$$

Therefore, we can compute numerically the integral $\tilde{I}_{p q, t}^{(1,3)}$. 


\section{Appendix E}

\section{Properties of the Green's functions used to calculate the $\mathrm{L}$ matrix.}

As it has been shown in Chapter 2, in the case of a cavity resonator, the scalar Green's functions (GFs) for the electric and magnetic scalar potential, and the dyadic Green's functions for the electric and magnetic vector potentials, may be expressed as:

$$
\begin{aligned}
g^{e}\left(\vec{r}, \vec{r}^{\prime}\right) & =\sum_{i}^{\infty} \frac{\phi_{i}(\vec{r}) \phi_{i}\left(\vec{r}^{\prime}\right)}{\mu_{i}^{2}} \\
g^{m}\left(\vec{r}, \vec{r}^{\prime}\right) & =\sum_{i}^{\infty} \frac{\psi_{i}(\vec{r}) \psi_{i}\left(\vec{r}^{\prime}\right)}{\nu_{i}^{2}} \\
\underline{G}^{A}\left(\vec{r}, \vec{r}^{\prime}\right) & =\sum_{i}^{\infty} \frac{\vec{E}_{i}(\vec{r}) \vec{E}_{i}\left(\vec{r}^{\prime}\right)}{k_{i}^{2}-k^{2}} \\
\underline{G}^{F}\left(\vec{r}, \vec{r}^{\prime}\right) & =\sum_{i}^{\infty} \frac{\vec{H}_{i}(\vec{r}) \vec{H}_{i}\left(\vec{r}^{\prime}\right)}{k_{i}^{2}-k^{2}}
\end{aligned}
$$

where $\phi_{i}(\vec{r})$ and $\mu_{i}$ are the scalar function and the eigenvalue associated to the $i$-th electric irrotational eigenvector of the cavity resonator; $\psi_{i}(\vec{r})$ and $\nu_{i}$ are the scalar function and the eigenvalue associated to the $i$-th magnetic irrotational eigenvector of the cavity resonator; $\vec{E}_{i}(\vec{r})$ and $\vec{H}_{i}(\vec{r})$ are, respectively, the electric and magnetic solenoidal eigenvectors of the cavity resonator, being $k_{i}$ its corresponding eigenvalue.

The $g^{e}$ function coincides with the Green's function for the electrostatic potential in the cavity according to the Coulomb's gauge, and it satisfies:

$$
\begin{aligned}
\nabla^{2} g^{e}\left(\vec{r}, \vec{r}^{\prime}\right) & =-\delta\left(\vec{r}, \vec{r}^{\prime}\right) & & \text { in } \quad V \\
g^{e} & =0 & & \text { on } S_{V}
\end{aligned}
$$


The equations satisfied by the magnetic scalar Green's function are dual:

$$
\begin{aligned}
\nabla^{2} g^{m}\left(\vec{r}, \vec{r}^{\prime}\right) & =-\delta\left(\vec{r}, \vec{r}^{\prime}\right) & & \text { in } \quad V \\
\frac{\partial g^{m}}{\partial n} & =0 & & \text { on } \quad S_{V}
\end{aligned}
$$

being $V$ the cavity volume and $S_{V}$ the cavity surface.

Moreover, we recall that the dyadic Green's function for the electric potential $\left(\underline{G}^{A}\right)$ is the solution of the following differential equation:

$$
\begin{aligned}
\nabla \times \nabla \times \underline{G}^{A}\left(\vec{r}, \vec{r}^{\prime}\right)-k^{2} \underline{G}^{A}\left(\vec{r}, \vec{r}^{\prime}\right) & =\underline{I} \delta\left(\vec{r}, \vec{r}^{\prime}\right)-\nabla \nabla^{\prime} g^{e}\left(\vec{r}, \vec{r}^{\prime}\right) & \text { in } \quad V \\
\widehat{n} \times \underline{G}^{A}\left(\vec{r}, \vec{r}^{\prime}\right) & =0 & \text { on } \quad S_{V}
\end{aligned}
$$

Since the singularity of the scalar GFs does not depend on the boundary conditions, it is the same for both the electric and magnetic scalar GFs:

$$
g_{s}\left(\vec{r}, \vec{r}^{\prime}\right)=\frac{1}{4 \pi R}
$$

where $\vec{R}=\vec{r}-\vec{r}^{\prime}$. Thus, the scalar Green's function may be decomposed as follows:

$$
\begin{aligned}
g^{e}\left(\vec{r}, \vec{r}^{\prime}\right) & =g_{s}\left(\vec{r}, \vec{r}^{\prime}\right)+g_{r}^{e}\left(\vec{r}, \vec{r}^{\prime}\right) \\
g^{m}\left(\vec{r}, \vec{r}^{\prime}\right) & =g_{s}\left(\vec{r}, \vec{r}^{\prime}\right)+g_{r}^{m}\left(\vec{r}, \vec{r}^{\prime}\right)
\end{aligned}
$$

where $g_{r}^{e}$ and $g_{r}^{m}$ are regular functions at $R=0$.

In the case of $G^{A}$, since the singularity is independent from the frequency, we have shown that the series expansion can be accelerated by extracting its zero-frequency limit, denoted as $\underline{G}_{o}^{A}$. Thus we can write:

$$
\underline{G}^{A}\left(\vec{r}, \vec{r}^{\prime}\right)=\underline{G}_{o}^{A}\left(\vec{r}, \vec{r}^{\prime}\right)+\underline{G}_{k}^{A}\left(\vec{r}, \vec{r}^{\prime}\right)
$$

being

$$
\left.\underline{G}_{o}^{A}\left(\vec{r}, \vec{r}^{\prime}\right) \equiv \underline{G}^{A}\left(\vec{r}, \vec{r}^{\prime}\right)\right|_{k=0} \quad \Rightarrow \quad \underline{G}_{o}^{A}\left(\vec{r}, \vec{r}^{\prime}\right)=\sum_{i}^{\infty} \frac{\vec{E}_{i}(\vec{r}) \vec{E}_{i}\left(\vec{r}^{\prime}\right)}{k_{i}^{2}}
$$

and,

$$
\underline{G}_{k}^{A}\left(\vec{r}, \vec{r}^{\prime}\right)=\underline{G}^{A}\left(\vec{r}, \vec{r}^{\prime}\right)-\underline{G}_{o}^{A}\left(\vec{r}, \vec{r}^{\prime}\right) \quad \Rightarrow \quad \underline{G}_{k}^{A}\left(\vec{r}, \vec{r}^{\prime}\right)=k^{2} \sum_{i}^{\infty} \frac{\vec{E}_{i}(\vec{r}) \vec{E}_{i}\left(\vec{r}^{\prime}\right)}{k_{i}^{2}\left(k_{i}^{2}-k^{2}\right)}
$$


Moreover, in Chapter 2, we have shown that the singularity of the dyadic GF can be extracted in closed form. Thus, the static term of the dyadic Green's function may be decomposed:

$$
\underline{G}_{o}^{A}\left(\vec{r}, \vec{r}^{\prime}\right)=\underline{G}_{s}^{o}\left(\vec{r}, \vec{r}^{\prime}\right)+\underline{G}_{o, r}^{A}\left(\vec{r}, \vec{r}^{\prime}\right)=\frac{1}{8 \pi R}\left(\underline{I}+\frac{\vec{R} \vec{R}}{R^{2}}\right)+\underline{G}_{o, r}^{A}\left(\vec{r}, \vec{r}^{\prime}\right)
$$

Therefore, we can finally write:

$$
\begin{aligned}
\underline{G}^{A}\left(\vec{r}, \vec{r}^{\prime}\right) & =\underline{G}_{o}^{A}\left(\vec{r}, \vec{r}^{\prime}\right)+\underline{G}_{k}^{A}\left(\vec{r}, \vec{r}^{\prime}\right)= \\
& =\frac{1}{8 \pi R}\left(\underline{I}+\frac{\vec{R} \vec{R}}{R^{2}}\right)+\underline{G}_{o, r}^{A}\left(\vec{r}, \vec{r}^{\prime}\right)+k^{2} \sum_{i}^{\infty} \frac{\vec{E}_{i}(\vec{r}) \vec{E}_{i}\left(\vec{r}^{\prime}\right)}{k_{i}^{2}\left(k_{i}^{2}-k^{2}\right)}
\end{aligned}
$$

The dual expression gives us the dyadic Green's function for the electric vector potential:

$$
\begin{aligned}
\underline{G}^{F}\left(\vec{r}, \vec{r}^{\prime}\right) & =\underline{G}_{o}^{F}\left(\vec{r}, \vec{r}^{\prime}\right)+\underline{G}_{k}^{F}\left(\vec{r}, \vec{r}^{\prime}\right)= \\
& =\frac{1}{8 \pi R}\left(\underline{I}+\frac{\vec{R} \vec{R}}{R^{2}}\right)+\underline{G}_{o, r}^{F}\left(\vec{r}, \vec{r}^{\prime}\right)+k^{2} \sum_{i}^{\infty} \frac{\vec{H}_{i}(\vec{r}) \vec{H}_{i}\left(\vec{r}^{\prime}\right)}{k_{i}^{2}\left(k_{i}^{2}-k^{2}\right)}
\end{aligned}
$$

where $\underline{G}_{o, r}^{A}$ and $\underline{G}_{o, r}^{F}$ are regular dyadic functions at $R=0$.

Next, we present some useful properties of the dyadic functions defined above. From equations (E.1a) and (E.1c), it can be easily observed the following:

$$
\begin{aligned}
g^{e}\left(\vec{r}^{\prime}, \vec{r}\right) & =\sum_{i}^{\infty} \frac{\phi_{i}\left(\vec{r}^{\prime}\right) \phi_{i}(\vec{r})}{\mu_{i}^{2}}=g^{e}\left(\vec{r}, \vec{r}^{\prime}\right) \\
\underline{G}^{A}\left(\vec{r}^{\prime}, \vec{r}\right) & =\sum_{i}^{\infty} \frac{\vec{E}_{i}\left(\vec{r}^{\prime}\right) \vec{E}_{i}(\vec{r})}{k_{i}^{2}-k^{2}}=\left(\underline{G}^{A}\left(\vec{r}, \vec{r}^{\prime}\right)\right)_{T}
\end{aligned}
$$

where $T$ denotes transpose. Analogously, the dual expressions can be obtained.

By applying the curl operator (C.14b) and (C.14d) to (E.1c) and (E.1d), we have:

$$
\begin{aligned}
& \nabla \times \underline{G}^{A}\left(\vec{r}, \vec{r}^{\prime}\right)=\sum_{i}^{\infty} \frac{\left(\nabla \times \vec{E}_{i}(\vec{r})\right) \vec{E}_{i}\left(\vec{r}^{\prime}\right)}{k_{i}^{2}-k^{2}}=\sum_{i}^{\infty} \frac{k_{i} \vec{H}_{i}(\vec{r}) \vec{E}_{i}\left(\vec{r}^{\prime}\right)}{k_{i}^{2}-k^{2}} \\
& \nabla \times \underline{G}^{F}\left(\vec{r}, \vec{r}^{\prime}\right)=\sum_{i}^{\infty} \frac{\left(\nabla \times \vec{H}_{i}(\vec{r})\right) \vec{H}_{i}\left(\vec{r}^{\prime}\right)}{k_{i}^{2}-k^{2}}=\sum_{i}^{\infty} \frac{k_{i} \vec{E}_{i}(\vec{r}) \vec{H}_{i}\left(\vec{r}^{\prime}\right)}{k_{i}^{2}-k^{2}} \\
& \nabla^{\prime} \times \underline{G}^{A}\left(\vec{r}, \vec{r}^{\prime}\right)=\sum_{i}^{\infty} \frac{\vec{E}_{i}(\vec{r})\left(\nabla^{\prime} \times \vec{E}_{i}\left(\vec{r}^{\prime}\right)\right)}{k_{i}^{2}-k^{2}}=\sum_{i}^{\infty} \frac{\left.k_{i} \vec{E}_{i}(\vec{r}) \vec{H}_{i}\left(\vec{r}^{\prime}\right)\right)}{k_{i}^{2}-k^{2}} \\
& \nabla^{\prime} \times \underline{G}^{F}\left(\vec{r}, \vec{r}^{\prime}\right)=\sum_{i}^{\infty} \frac{\vec{H}_{i}(\vec{r})\left(\nabla^{\prime} \times \vec{H}_{i}\left(\vec{r}^{\prime}\right)\right)}{k_{i}^{2}-k^{2}}=\sum_{i}^{\infty} \frac{\left.k_{i} \vec{H}_{i}(\vec{r}) \vec{E}_{i}\left(\vec{r}^{\prime}\right)\right)}{k_{i}^{2}-k^{2}}
\end{aligned}
$$

where we have used $\nabla \times \vec{E}_{i}(\vec{r})=k_{i} \vec{H}_{i}(\vec{r})$ and $\nabla \times \vec{H}_{i}(\vec{r})=k_{i} \vec{E}_{i}(\vec{r})$. From expressions (E.15), we can conclude:

$$
\begin{aligned}
& \nabla \times \underline{G}^{A}\left(\vec{r}, \vec{r}^{\prime}\right)=\nabla^{\prime} \times \underline{G}^{F}\left(\vec{r}, \vec{r}^{\prime}\right) \\
& \nabla^{\prime} \times \underline{G}^{A}\left(\vec{r}, \vec{r}^{\prime}\right)=\nabla \times \underline{G}^{F}\left(\vec{r}, \vec{r}^{\prime}\right)
\end{aligned}
$$


It is also useful to calculate the following expressions:

$$
\begin{gathered}
\nabla \times \nabla \times \underline{G}^{A}\left(\vec{r}, \vec{r}^{\prime}\right)=\sum_{i}^{\infty} \frac{\left(\nabla \times \nabla \times \vec{E}_{i}(\vec{r})\right) \vec{E}_{i}\left(\vec{r}^{\prime}\right)}{k_{i}^{2}-k^{2}}=\sum_{i}^{\infty} k_{i}^{2} \frac{\vec{E}_{i}(\vec{r}) \vec{E}_{i}\left(\vec{r}^{\prime}\right)}{k_{i}^{2}-k^{2}} \\
\nabla^{\prime} \times \nabla^{\prime} \times \underline{G}^{A}\left(\vec{r}, \vec{r}^{\prime}\right)=\sum_{i}^{\infty} \frac{\vec{E}_{i}(\vec{r})\left(\nabla^{\prime} \times \nabla^{\prime} \times \vec{E}_{i}\left(\vec{r}^{\prime}\right)\right)}{k_{i}^{2}-k^{2}}=\sum_{i}^{\infty} k_{i}^{2} \frac{\vec{E}_{i}(\vec{r}) \vec{E}_{i}\left(\vec{r}^{\prime}\right)}{k_{i}^{2}-k^{2}}
\end{gathered}
$$

Comparing these last expressions, we can wirte:

$$
\nabla \times \nabla \times \underline{G}^{A}\left(\vec{r}, \vec{r}^{\prime}\right)=\nabla^{\prime} \times \nabla^{\prime} \times \underline{G}^{A}\left(\vec{r}, \vec{r}^{\prime}\right)
$$

It can be easily verified that the dual expression also holds,

$$
\nabla \times \nabla \times \underline{G}^{F}\left(\vec{r}, \vec{r}^{\prime}\right)=\nabla^{\prime} \times \nabla^{\prime} \times \underline{G}^{F}\left(\vec{r}, \vec{r}^{\prime}\right)
$$

By proceding analogously with the definition of the static dyadic defined in (E.8) and its dual expression, we can also obtain the following:

$$
\begin{aligned}
\nabla \times \underline{G}_{o}^{A}\left(\vec{r}, \vec{r}^{\prime}\right) & =\nabla^{\prime} \times \underline{G}_{o}^{F}\left(\vec{r}, \vec{r}^{\prime}\right) \\
\nabla^{\prime} \times \underline{G}_{o}^{A}\left(\vec{r}, \vec{r}^{\prime}\right) & =\nabla \times \underline{G}_{o}^{F}\left(\vec{r}, \vec{r}^{\prime}\right) \\
\nabla \times \nabla \times \underline{G}_{o}^{A}\left(\vec{r}, \vec{r}^{\prime}\right) & =\nabla^{\prime} \times \nabla^{\prime} \times \underline{G}_{o}^{A}\left(\vec{r}, \vec{r}^{\prime}\right) \\
\nabla \times \nabla \times \underline{G}_{o}^{F}\left(\vec{r}, \vec{r}^{\prime}\right) & =\nabla^{\prime} \times \nabla^{\prime} \times \underline{G}_{o}^{F}\left(\vec{r}, \vec{r}^{\prime}\right)
\end{aligned}
$$

\section{E.1 Singular dyadic term of the static part of the Green's function for the potential vector}

In this section, we focus our attention in the singular dyadic term of the Green's function for the potential vector:

$$
\underline{G}_{s}^{o}\left(\vec{r}, \vec{r}^{\prime}\right)=\frac{1}{8 \pi R}\left(\underline{I}+\frac{\vec{R} \vec{R}}{R^{2}}\right)
$$

Next, we will demonstrate that this singular dyadic satisfies the following properties: 


$$
\begin{aligned}
\underline{G}_{s}^{o}(\vec{R}) & =-\nabla \times \nabla \times \frac{R \underline{I}}{8 \pi} \\
& =-\nabla^{\prime} \times \nabla^{\prime} \times \frac{R \underline{I}}{8 \pi} \\
\nabla \times \underline{G}_{s}^{o}(\vec{R}) & =-\nabla \times \nabla \times \frac{\vec{R} \times \underline{I}}{8 \pi R} \\
\nabla^{\prime} \times \underline{G}_{s}^{o}(\vec{R}) & =-\nabla^{\prime} \times \nabla^{\prime} \times \frac{\vec{R} \times \underline{I}}{8 \pi R} \\
\nabla \times \underline{G}_{s}^{o}(\vec{R}) & =\nabla^{\prime} \times \underline{G}_{s}^{o}(\vec{R})=-\left(\nabla \times \underline{G}_{s}^{o}(\vec{R})\right)_{T} \\
\nabla \times \nabla \times \underline{G}_{s}^{o}(\vec{R}) & =\nabla^{\prime} \times \nabla^{\prime} \times \underline{G}_{s}^{o}(\vec{R})=\left(\nabla \times \nabla \times \underline{G}_{s}^{o}(\vec{R})\right)_{T}
\end{aligned}
$$

The curl operator and the singular Dyadic in the coordinate system $\{\widehat{x}\}$

Firstly, in order to demonstrate (E.23a) and (E.23b), let apply the identity (C.6) to obtain the following expression:

$$
\nabla \times \frac{R \underline{I}}{8 \pi}=\frac{1}{8 \pi}(\nabla R) \times \underline{I}+R \nabla \times \underline{I}=\frac{\vec{R} \times \underline{I}}{8 \pi R}
$$

Thus, we can also write:

$$
\begin{aligned}
\nabla \times \frac{\vec{R} \times \underline{I}}{8 \pi R} & =\frac{1}{8 \pi}\left[\left(\nabla R^{-1}\right) \times(\vec{R} \times \underline{I})+R^{-1} \nabla \times(\vec{R} \times \underline{I})\right] \\
& =\frac{1}{8 \pi}\left[-\frac{\vec{R}}{R^{3}} \times(\vec{R} \times \underline{I})+R^{-1} \nabla \times(\vec{R} \times \underline{I})\right]
\end{aligned}
$$

To develop the right hand side of (E.25), we have to evaluate the following identities:

$$
\vec{a} \times(\vec{b} \times \underline{C})=\vec{b}(\vec{a} \cdot \underline{C})-(\vec{a} \cdot \vec{b}) \underline{C} \quad \Rightarrow \quad \vec{R} \times(\vec{R} \times \underline{I})=\vec{R} \vec{R}-R^{2} \underline{I}
$$

and

$$
\nabla \times(\vec{R} \times \underline{I})=-2 \underline{I}
$$

To achieve this last result, we have considered that

$$
\nabla \times(\vec{R} \times \underline{I})=\sum_{j} \nabla \times\left(\vec{R} \times \vec{I}_{j}\right) \widehat{x}_{j}
$$

Since,

$$
\vec{R} \times \vec{I}_{j}=\sum_{m q s} \epsilon_{m q s} R_{q}\left(I_{j}\right)_{s} \widehat{x}_{m}=\sum_{m q s} \epsilon_{m q s} R_{q} \delta_{j s} \widehat{x}_{m}=\sum_{m q} \epsilon_{m q j} R_{q} \widehat{x}_{m},
$$


where $\epsilon_{i j k}$ is the Levi-Civita symbol. Then we have

$$
\begin{aligned}
\nabla \times\left(\vec{R} \times \vec{I}_{j}\right) & =\sum_{p t l} \epsilon_{p t l} \partial_{x t}\left(\vec{R} \times \vec{I}_{j}\right)_{l} \widehat{x}_{p}=\sum_{p t l q} \epsilon_{p t l} \partial_{x_{t}} \epsilon_{l q j} R_{q} \widehat{x}_{p}=\sum_{p t l q} \epsilon_{p t l} \epsilon_{l q j} \delta_{t q} \widehat{x}_{p} \\
& =\sum_{p t l} \epsilon_{p t l} \epsilon_{l t j} \widehat{x}_{p}=-\sum_{p t l} \epsilon_{p t l} \epsilon_{j t l} \widehat{x}_{p}=-2 \sum_{p} \delta_{p j} \widehat{x}_{p}=-2 \widehat{x}_{j}
\end{aligned}
$$

where we have used the identity $\sum_{t l} \epsilon_{p t l} \epsilon_{j t l}=2 \delta_{p j}$.

Thus, by substituting equation (E.30) in (E.28), we obtain the identity (E.27). And finally introducing the expressions (E.27) and (E.26) in (E.25), we obtain:

$$
\begin{aligned}
\nabla \times\left(\frac{\vec{R} \times \underline{I}}{8 \pi R}\right) & =\frac{1}{8 \pi}\left[R^{-3}\left(-\vec{R} \vec{R}+R^{2} \underline{I}\right)-2 R^{-1} \underline{I}\right] \\
& =-\frac{1}{8 \pi R}\left(\frac{\vec{R} \vec{R}}{R^{2}}+\underline{I}\right)=-\underline{G}_{s}^{o}(\vec{R})
\end{aligned}
$$

And therefore, making use of expressions (E.24) and (E.31), we can demonstrate (E.23b) and the first term of the expression (E.23a):

$$
\begin{gathered}
\underline{G}_{s}^{o}(\vec{R})=-\nabla \times \nabla \times \frac{R \underline{I}}{8 \pi} \\
\nabla \times \underline{G}_{s}^{o}(\vec{R})=-\nabla \times \nabla \times \frac{\vec{R} \times \underline{I}}{8 \pi R}
\end{gathered}
$$

In addition, by using (C.6) and (E.22), we can write the curl of the singular dyadic as follows:

$$
\begin{aligned}
\nabla \times \underline{G}_{s}^{o}(\vec{R}) & =\nabla \times\left[\frac{1}{8 \pi R}\left(\underline{I}+\frac{\vec{R} \vec{R}}{R^{2}}\right)\right] \\
& =\frac{1}{8 \pi}\left[\left(\nabla R^{-1}\right) \times\left(\underline{I}+\frac{\vec{R} \vec{R}}{R^{2}}\right)+R^{-1} \nabla \times\left(\underline{I}+\frac{\vec{R} \vec{R}}{R^{2}}\right)\right]
\end{aligned}
$$

Next, we evaluate the term $\nabla \times\left(\underline{I}+\frac{\vec{R} \vec{R}}{R^{2}}\right)$,

$$
\nabla \times\left(\underline{I}+\frac{\vec{R} \vec{R}}{R^{2}}\right)=\nabla \times \frac{\vec{R} \vec{R}}{R^{2}}=\left(\nabla R^{-2}\right) \times \vec{R} \vec{R}+R^{-2} \nabla \times \vec{R} \vec{R}
$$

Taking into account the following identities:

$$
\left(\nabla R^{-2}\right) \times \vec{R} \vec{R}=-2 \frac{\vec{R}}{R^{4}} \times \vec{R} \vec{R}=0
$$


and,

$$
\begin{aligned}
\nabla \times \vec{R} \vec{R} & =\sum_{j} \nabla \times\left(\vec{R} R_{j}\right) \widehat{x}_{j}^{\prime}=\sum_{j}\left(\nabla R_{j} \times \vec{R}+R_{j} \nabla \times \vec{R}\right) \widehat{x}_{j}^{\prime} \\
& =\sum_{j}\left(\widehat{x}_{j} \times \vec{R}\right) \widehat{x}_{j}^{\prime}=\sum_{j}-\left(\vec{R} \times \widehat{x}_{j}\right) \widehat{x}_{j}^{\prime}=-\vec{R} \times \underline{I}
\end{aligned}
$$

where we have used, $\nabla R_{j}=\sum_{i} \partial_{x_{i}}\left(x_{j}-x_{j}^{\prime}\right) \widehat{x}_{i}=\sum_{i} \delta_{i j} \widehat{x}_{i}=\widehat{x}_{j}$ and $\nabla \times \vec{R}=0$. Then we have:

$$
\nabla \times\left(\underline{I}+\frac{\vec{R} \vec{R}}{R^{2}}\right)=-\frac{\vec{R} \times \underline{I}}{R^{2}}
$$

And thus, introducing (E.37) in (E.33), we obtain:

$$
\nabla \times \underline{G}_{s}^{o}(\vec{R})=\frac{1}{8 \pi}\left[-\frac{\vec{R}}{R^{3}} \times\left(\underline{I}+\frac{\vec{R} \vec{R}}{R^{2}}\right)-R^{-1} \frac{\vec{R} \times \underline{I}}{R^{2}}\right]=-\frac{\vec{R} \times \underline{I}}{4 \pi R^{3}}
$$

where we have used

$$
\nabla R^{-1}=-\frac{\vec{R}}{R^{3}}
$$

Making use of these results, we can obtain the following expression:

$$
\begin{aligned}
& \nabla \times \nabla \times \underline{G}_{s}^{o}(\vec{R})=\nabla \times\left(-\frac{\vec{R} \times \underline{I}}{4 \pi R^{3}}\right) \\
& =-\frac{1}{4 \pi}\left[\left(\nabla R^{-3}\right) \times(\vec{R} \times \underline{I})+R^{-3} \nabla \times((\vec{R} \times \underline{I}))\right] \\
& =-\frac{1}{4 \pi}\left[-3 \frac{\vec{R}}{R^{5}} \times(\vec{R} \times \underline{I})+R^{-3} \nabla \times(\vec{R} \times \underline{I})\right] \\
& =-\frac{1}{4 \pi}\left[-3 R^{-5}\left(\vec{R} \vec{R}-R^{2} \underline{I}\right)-2 R^{-3} \underline{I}\right]=\frac{1}{4 \pi R^{3}}\left[\underline{I}-3 \frac{\vec{R} \vec{R}}{R^{2}}\right]
\end{aligned}
$$

Furthermore, using (C.6), it is easy to verify that

$$
\nabla \times \frac{I}{R}=\nabla R^{-1} \times \underline{I}
$$

and thus, we can also write:

$$
\begin{aligned}
\nabla \times \nabla \times \underline{G}_{s}^{o}(\vec{R}) & =\nabla \times\left(-\frac{\vec{R} \times \underline{I}}{4 \pi R^{3}}\right)=\frac{1}{4 \pi} \nabla \times\left(\nabla R^{-1} \times \underline{I}\right)=\nabla \times \nabla \times \frac{\underline{I}}{4 \pi R} \\
& =\nabla \nabla \frac{1}{4 \pi R}-\frac{\underline{I}}{4 \pi} \nabla^{2} \frac{1}{R}=-\nabla \nabla^{\prime} \frac{1}{4 \pi R}+\underline{I} \delta(\vec{R})
\end{aligned}
$$


where $\delta(\vec{R})$ is the Dirac delta function. To obtain this last result, we have used the dyadic identity $\nabla \times \nabla \times(\psi \underline{I})=\nabla \nabla \psi-\underline{I} \nabla^{2} \psi$, being $\psi$ a scalar fucntion, and the equation:

$$
\nabla^{2} \frac{1}{R}=-4 \pi \delta(\vec{R})
$$

\section{The curl operator and the singular Dyadic in the coordinate system $\left\{\widehat{x}^{\prime}\right\}$}

In this case, in order to demonstrate (E.23a) and (E.23c), we apply the identity (C.15) to obtain the following expressions:

$$
\nabla^{\prime} \times \frac{R \underline{I}}{8 \pi}=\frac{1}{8 \pi}\left[R \nabla^{\prime} \times \underline{I}-\underline{I} \times\left(\nabla^{\prime} R\right)\right]=\frac{\underline{I} \times \vec{R}}{8 \pi R}=\frac{\vec{R} \times \underline{I}}{8 \pi R}
$$

and

$$
\begin{aligned}
\nabla^{\prime} \times \frac{\underline{I} \times \vec{R}}{8 \pi R} & =\frac{1}{8 \pi}\left[R^{-1} \nabla^{\prime} \times(\underline{I} \times \vec{R})-(\underline{I} \times \vec{R}) \times\left(\nabla^{\prime} R^{-1}\right)\right] \\
& =\frac{1}{8 \pi}\left[R^{-1} \nabla^{\prime} \times(\underline{I} \times \vec{R})-(\underline{I} \times \vec{R}) \times \frac{\vec{R}}{R^{3}}\right]
\end{aligned}
$$

where we have used $\nabla^{\prime} R^{-1}=\vec{R} / R^{3}$ and $\vec{a} \times \underline{I}=\underline{I} \times \vec{a}$. To develop the right hand side of the last expression, we have to evaluate the following identities:

$$
(\underline{I} \times \vec{R}) \times \vec{R}=(\vec{R} \times \underline{I}) \times \vec{R}=\vec{R} \times(\underline{I} \times \vec{R})=\vec{R} \times(\vec{R} \times \underline{I})=\vec{R} \vec{R}-R^{2} \underline{I}
$$

and

$$
\nabla^{\prime} \times(\underline{I} \times \vec{R})=-2 \underline{I}
$$

To achieve this last result, we have taken into consideration that

$$
\nabla^{\prime} \times(\underline{I} \times \vec{R})=\sum_{j} \widehat{x}_{j}^{\prime} \nabla^{\prime} \times\left(\vec{I}_{j} \times \vec{R}\right)
$$

Since,

$$
\vec{I}_{j} \times \vec{R}=\sum_{m q s} \epsilon_{m q s}\left(I_{j}\right)_{q} R_{s} \widehat{x}_{m}^{\prime}=\sum_{m q s} \epsilon_{m q s} R_{s} \delta_{j q} \widehat{x}_{m}^{\prime}=\sum_{m s} \epsilon_{m j s} R_{s} \widehat{x}_{m}^{\prime}
$$

then we have

$$
\begin{aligned}
\nabla^{\prime} \times\left(\vec{I}_{j} \times \vec{R}\right) & =\sum_{p t l} \epsilon_{p t l} \partial_{x_{t}^{\prime}}\left(\vec{I}_{j} \times \vec{R}\right)_{l} \widehat{x}_{p}^{\prime}=\sum_{p t l s} \epsilon_{p t l} \partial_{x_{t}^{\prime}} \epsilon_{l j s} R_{s} \widehat{x}_{p}^{\prime}=-\sum_{p t l s} \epsilon_{p t l} \epsilon_{l j s} \delta_{t s} \widehat{x}_{p}^{\prime} \\
& =-\sum_{p t l} \epsilon_{p t l} \epsilon_{l j t} \widehat{x}_{p}^{\prime}=-\sum_{p t l} \epsilon_{p t l} \epsilon_{j t l} \widehat{x}_{p}^{\prime}=-2 \sum_{p} \delta_{p j} \widehat{x}_{p}^{\prime}=-2 \widehat{x}_{j}^{\prime}
\end{aligned}
$$


By substituting equation (E.48) in (E.46), we obtain the identity (E.45). Thus, introducing the expressions (E.45) and (E.44) in (E.43), we can write:

$$
\begin{aligned}
\nabla^{\prime} \times\left(\frac{\vec{R} \times \underline{I}}{8 \pi R}\right) & =\frac{1}{8 \pi}\left[-2 R^{-1} \underline{I}-R^{-3}\left(\vec{R} \vec{R}-R^{2} \underline{I}\right)\right] \\
& =-\frac{1}{8 \pi R}\left(\frac{\vec{R} \vec{R}}{R^{2}}+\underline{I}\right)=-\underline{G}_{s}^{o}(\vec{R})
\end{aligned}
$$

And therefore, making use of expressions (E.42) and (E.49), we can demonstrate (E.23c) and the second term of the expression (E.23a):

$$
\begin{gathered}
\underline{G}_{s}^{o}(\vec{R})=-\nabla^{\prime} \times \nabla^{\prime} \times \frac{R \underline{I}}{8 \pi} \\
\nabla^{\prime} \times \underline{G}_{s}^{o}(\vec{R})=-\nabla^{\prime} \times \nabla^{\prime} \times \frac{\underline{I} \times \vec{R}}{8 \pi R}
\end{gathered}
$$

In addition, by using (C.15) and (E.22), we can write $\nabla^{\prime} \times \underline{G}_{s}^{o}(\vec{R})$ as follows:

$$
\begin{aligned}
\nabla^{\prime} \times \underline{G}_{s}^{o}(\vec{R}) & =\nabla^{\prime} \times\left[\frac{1}{8 \pi R}\left(\underline{I}+\frac{\vec{R} \vec{R}}{R^{2}}\right)\right] \\
& =\frac{1}{8 \pi}\left[R^{-1} \nabla^{\prime} \times\left(\underline{I}+\frac{\vec{R} \vec{R}}{R^{2}}\right)-\left(\underline{I}+\frac{\vec{R} \vec{R}}{R^{2}}\right) \times\left(\nabla^{\prime} R^{-1}\right)\right] \\
& =\frac{1}{8 \pi}\left[R^{-1} \nabla^{\prime} \times \frac{\vec{R} \vec{R}}{R^{2}}-\underline{I} \times\left(\nabla^{\prime} R^{-1}\right)\right]
\end{aligned}
$$

And using again (C.15), we have:

$$
\nabla^{\prime} \times\left(\frac{\vec{R} \vec{R}}{R^{2}}\right)=R^{-2} \nabla^{\prime} \times(\vec{R} \vec{R})-\vec{R} \vec{R} \times \nabla^{\prime} R^{-2}=R^{-2} \nabla^{\prime} \times(\vec{R} \vec{R})=-\frac{I \times \vec{R}}{R^{2}}
$$

where we have used,

$$
\begin{aligned}
\nabla^{\prime} \times \vec{R} \vec{R} & =\sum_{i} \widehat{x}_{i} \nabla^{\prime} \times\left(R_{i} \vec{R}\right)=\sum_{i} \widehat{x}_{i}\left(\left(\nabla^{\prime} R_{i}\right) \times \vec{R}+R_{i} \nabla^{\prime} \times \vec{R}\right) \\
& =\sum_{i} \widehat{x}_{i}\left(-\widehat{x}_{i}^{\prime} \times \vec{R}\right)=-\underline{I} \times \vec{R}
\end{aligned}
$$

Using $\nabla^{\prime} R^{-1}=\vec{R} / R^{3}$, and introducing (E.52) in (E.51), we finally obtain

$$
\nabla^{\prime} \times \underline{G}_{s}^{o}(\vec{R})=\frac{1}{8 \pi}\left[-R^{-1} \frac{\underline{I} \times \vec{R}}{R^{2}}-\underline{I} \times \frac{\vec{R}}{R^{3}}\right]=-\frac{\underline{I} \times \vec{R}}{4 \pi R^{3}}
$$


Therefore, since $\vec{R} \times \underline{I}=\underline{I} \times \vec{R}$, by comparing (E.38) and (E.54), we demonstrate that the property expressed by (E.23d) is satisfied. Furthermore, using the dyadic identity, $\vec{a} \times \underline{C}=-\left(\underline{C}_{T} \times \vec{a}\right)_{T}$, we can write

$$
\left(\nabla \times \underline{G}_{s}^{o}(\vec{R})\right)_{T}=-\frac{(\vec{R} \times \underline{I})^{T}}{4 \pi R^{3}}=\frac{I \times \vec{R}}{4 \pi R^{3}}=-\nabla \times \underline{G}_{s}^{o}(\vec{R})
$$

Finally, by using these last results, we can obtain another useful expression:

$$
\begin{aligned}
& \nabla^{\prime} \times \nabla^{\prime} \times \underline{G}_{s}^{o}(\vec{R})=\nabla^{\prime} \times\left(-\frac{\underline{I} \times \vec{R}}{4 \pi R^{3}}\right) \\
& =-\frac{1}{4 \pi}\left[\left(R^{-3} \nabla^{\prime} \times(\underline{I} \times \vec{R})-(\underline{I} \times \vec{R}) \times\left(\nabla^{\prime} R^{-3}\right)\right]\right. \\
& =-\frac{1}{4 \pi}\left[R^{-3} \nabla^{\prime} \times(\underline{I} \times \vec{R})-3(\underline{I} \times \vec{R}) \times \frac{\vec{R}}{R^{5}}\right] \\
& =-\frac{1}{4 \pi}\left[-2 R^{-3} \underline{I}-3 R^{-5}\left(\vec{R} \vec{R}-R^{2} \underline{I}\right)\right]=\frac{1}{4 \pi R^{3}}\left[\underline{I}-3 \frac{\vec{R} \vec{R}}{R^{2}}\right]
\end{aligned}
$$

It is easily observed that this expression is a symmetric dyadic. Moreover by comparing it with equation (E.40), the identity (E.23e) is validated.

\section{E.2 Regular dyadic term of the static part of the Green's function for the potential vector}

In this section, we focus our attention on the regular dyadic term of the static part of the Green's function for both the electric and magnetic potential vectors,

$$
\begin{aligned}
& \underline{G}_{o, r}^{A}\left(\vec{r}, \vec{r}^{\prime}\right)=\underline{G}_{o}^{A}\left(\vec{r}, \vec{r}^{\prime}\right)-\underline{G}_{s}^{o}\left(\vec{r}, \vec{r}^{\prime}\right) \\
& \underline{G}_{o, r}^{F}\left(\vec{r}, \vec{r}^{\prime}\right)=\underline{G}_{o}^{F}\left(\vec{r}, \vec{r}^{\prime}\right)-\underline{G}_{s}^{o}\left(\vec{r}, \vec{r}^{\prime}\right)
\end{aligned}
$$

Applying the curl opertaor to these expressions and making use of (E.21), (E.23d) and (E.23e), we can easily conlcude the following:

$$
\begin{aligned}
\nabla \times \underline{G}_{o, r}^{A}\left(\vec{r}, \vec{r}^{\prime}\right) & =\nabla^{\prime} \times \underline{G}_{o, r}^{F}\left(\vec{r}, \vec{r}^{\prime}\right) \\
\nabla^{\prime} \times \underline{G}_{o, r}^{A}\left(\vec{r}, \vec{r}^{\prime}\right) & =\nabla \times \underline{G}_{o, r}^{F}\left(\vec{r}, \vec{r}^{\prime}\right) \\
\nabla \times \nabla \times \underline{G}_{o, r}^{A}\left(\vec{r}, \vec{r}^{\prime}\right) & =\nabla^{\prime} \times \nabla^{\prime} \times \underline{G}_{o, r}^{A}\left(\vec{r}, \vec{r}^{\prime}\right) \\
\nabla \times \nabla \times \underline{G}_{o, r}^{F}\left(\vec{r}, \vec{r}^{\prime}\right) & =\nabla^{\prime} \times \nabla^{\prime} \times \underline{G}_{o, r}^{F}\left(\vec{r}, \vec{r}^{\prime}\right)
\end{aligned}
$$

Next, if we particularise equation (E.4a) for $k=0$, we have:

$$
\nabla \times \nabla \times \underline{G}_{o}^{A}\left(\vec{r}, \vec{r}^{\prime}\right)=\underline{I} \delta\left(\vec{r}, \vec{r}^{\prime}\right)-\nabla \nabla^{\prime} g^{e}\left(\vec{r}, \vec{r}^{\prime}\right)
$$


E.2 Regular dyadic term of the static part of the Green's function for the potential vector

and introducing now (E.6) and (E.10) into this differential equation, we may write

$$
\nabla \times \nabla \times \underline{G}_{s}^{o}(\vec{R})+\nabla \times \nabla \times \underline{G}_{o, r}^{A}\left(\vec{r}, \vec{r}^{\prime}\right)=\underline{I} \delta\left(\vec{r}, \vec{r}^{\prime}\right)-\nabla \nabla^{\prime} \frac{1}{4 \pi R}-\nabla \nabla^{\prime} g_{r}^{e}\left(\vec{r}, \vec{r}^{\prime}\right)
$$

Finally, taking into account (E.41), we can conclude the following:

$$
\nabla \times \nabla \times \underline{G}_{o, r}^{A}\left(\vec{r}, \vec{r}^{\prime}\right)=-\nabla \nabla^{\prime} g_{r}^{e}\left(\vec{r}, \vec{r}^{\prime}\right)
$$

Moreover, due to (E.59c), we can also write:

$$
\nabla^{\prime} \times \nabla^{\prime} \times \underline{G}_{o, r}^{A}\left(\vec{r}, \vec{r}^{\prime}\right)=-\nabla \nabla^{\prime} g_{r}^{e}\left(\vec{r}, \vec{r}^{\prime}\right)
$$

It is easy to obtain the dual expression following an analogous procedure:

$$
\nabla \times \nabla \times \underline{G}_{o, r}^{F}\left(\vec{r}, \vec{r}^{\prime}\right)=-\nabla \nabla^{\prime} g_{r}^{m}\left(\vec{r}, \vec{r}^{\prime}\right)
$$




\section{Appendix F}

\section{Auxiliary series and functions used in the aperture of the access ports}

\section{F.1 Auxiliary series used in the aperture of the access ports}

As described in Chapter 4, to proceed with the apertures of the lateral and top access ports of the rectangular cavity, we need to calculate the matrices $G_{m, n}^{(\gamma, \xi)}, T_{m, n}^{(\gamma, \xi)}$ and $W_{m, n}^{(\xi)}$. For this purpose, we have made use of the following series [120]:

$$
\begin{aligned}
& \sum_{n_{z}=0}^{\infty} \frac{\epsilon_{n_{z}} \cos \left(k_{z} z\right)}{k_{t}^{2}+k_{z}^{2}}=\frac{l_{z}}{k_{t}} \frac{\cosh \left[k_{t}\left(l_{z}-z\right)\right]}{\sinh \left(k_{t} l_{z}\right)} \\
& \sum_{n_{z}=0}^{\infty}(-1)^{n_{z}} \frac{\epsilon_{n_{z}} \cos \left(k_{z} z\right)}{k_{t}^{2}+k_{z}^{2}}=\frac{l_{z}}{k_{t}} \frac{\cosh \left(k_{t} z\right)}{\sinh \left(k_{t} l_{z}\right)} \\
& \sum_{n_{z}=0}^{\infty} \frac{k_{z} \sin \left(k_{z} z\right)}{k_{t}^{2}+k_{z}^{2}}=\frac{l_{z}}{2} \frac{\sinh \left[k_{t}\left(l_{z}-z\right)\right]}{\sinh \left(k_{t} l_{z}\right)} \\
& \sum_{n_{z}=0}^{\infty}(-1)^{n_{z}} \frac{k_{z} \sin \left(k_{z} z\right)}{k_{t}^{2}+k_{z}^{2}}=-\frac{l_{z}}{2} \frac{\sinh \left(k_{t} z\right)}{\sinh \left(k_{t} l_{z}\right)} \\
& \sum_{n_{z}=0}^{\infty} \frac{k_{z}^{2}}{\left(k_{t}^{2}+k_{z}^{2}\right)^{2}}=\frac{l_{z}}{4 k_{t}}\left(\operatorname{coth}\left(k_{t} l_{z}\right)-\frac{k_{t} l_{z}}{\sinh ^{2}\left(k_{t} l_{z}\right)}\right) \\
& \sum_{n_{z}=0}^{\infty}(-1)^{n_{z}} \frac{k_{z}^{2}}{\left(k_{t}^{2}+k_{z}^{2}\right)^{2}}=\frac{l_{z}}{4 k_{t} \sinh \left(k_{t} l_{z}\right)}\left(1-\frac{k_{t} l_{z}}{\tanh \left(k_{t} l_{z}\right)}\right)
\end{aligned}
$$

In all these expressions we have that $k_{z}=\frac{n_{z} \pi}{l_{z}}$. 


\section{F.2 Auxiliary functions used in the aperture of the access ports}

The expressions of the matrices $G_{m, n}^{(\gamma, \xi)}$ and $T_{m, n}^{(\gamma, \xi)}$ obtained in the equations (4.164) and (4.180)-(4.183) of Chapter 4 have been written in terms of the following functions:

$$
\begin{aligned}
& \rho_{m, n}^{(\gamma, \xi)}=\left\{\begin{array}{l}
\sqrt{\epsilon_{m_{x_{\gamma}}} \epsilon_{n_{x_{\xi}}}}, \text { if } \gamma=1,2 \mathrm{y} \xi=3,4 \\
\sqrt{\epsilon_{m_{y_{\gamma}}} \epsilon_{n_{x_{\xi}}}}, \text { if } \gamma=1,2 \mathrm{y} \xi=5 \\
\sqrt{\epsilon_{m_{y_{\gamma}}} \epsilon_{n_{y_{\xi}}}}, \text { if } \gamma=3,4 \mathrm{y} \xi=5
\end{array}\right. \\
& \psi_{m, n}^{(\gamma, \xi)}=\left\{\begin{array}{l}
\left(-\nu_{\gamma}\right)^{n_{x_{\xi}}} \nu_{\xi}^{m_{x_{\gamma}}} \delta_{m_{y_{\gamma}}, n_{y_{\xi}}}, \text { if } \gamma=1,2 \mathrm{y} \xi=3,4 \\
\left(-\nu_{\gamma}\right)^{n_{x_{\xi}}}\left(-\nu_{\xi}\right)^{m_{y_{\gamma}}} \delta_{m_{x_{\gamma}}, n_{y_{\xi}}}, \text { if } \gamma=1,2 \mathrm{y} \xi=5 \\
\nu_{\gamma}^{n_{y_{\xi}}}\left(-\nu_{\xi}\right)^{m_{y_{\gamma}}} \delta_{m_{x_{\gamma}}, n_{x_{\xi}}}, \text { if } \gamma=3,4 \mathrm{y} \xi=5
\end{array}\right. \\
& \chi_{m, n}^{(\gamma, \xi)}=\left\{\begin{array}{l}
k_{n_{y_{\xi}}}^{2} \frac{\epsilon_{n_{y_{\xi}}}\left(\epsilon_{n_{y_{\xi}}}-1\right)}{\epsilon_{m_{x_{\gamma}}}}, \text { if } \gamma=1,2 \mathrm{y} \xi=3,4 \\
k_{n_{y_{\xi}}}^{2} \frac{\epsilon_{n_{\xi}}\left(\epsilon_{n_{y_{\xi}}}-1\right)}{\epsilon_{m_{y_{\gamma}}}}, \text { if } \gamma=1,2 \mathrm{y} \xi=5 \\
k_{n_{x_{\xi}}}^{2} \frac{\epsilon_{n_{x_{\xi}}}\left(\epsilon_{n_{x_{\xi}}}-1\right)}{\epsilon_{n_{y_{\gamma}}}}, \text { if } \gamma=3,4 \mathrm{y} \xi=5
\end{array}\right. \\
& \varphi_{m, n}^{(\gamma, \xi)}=\left\{\begin{array}{l}
\epsilon_{m_{x_{\gamma}}}-1, \text { if } \gamma=1,2 \mathrm{y} \xi=3,4 \\
\epsilon_{m_{y_{\gamma}}}-1, \text { if } \gamma=1,2,3,4 \mathrm{y} \xi=5
\end{array}\right. \\
& \zeta_{m, n}^{(\gamma, \xi)}=\left\{\begin{array}{l}
k_{n_{y_{\xi}} \sqrt{\epsilon_{n_{x_{\xi}}}}}, \text { if } \gamma=1,2 \mathrm{y} \xi=3,4,5 \\
k_{n_{x_{\xi}} \sqrt{\epsilon_{n_{y_{\xi}}}},} \text { if } \gamma=3,4 \mathrm{y} \xi=5
\end{array}\right. \\
& \theta_{m, n}^{(\gamma, \xi)}=\left\{\begin{array}{l}
\sqrt{\epsilon_{m_{x \gamma}}}, \text { if } \gamma=1,2 \mathrm{y} \xi=3,4 \\
\sqrt{\epsilon_{m_{y_{\gamma}}}}, \text { if } \gamma=1,2 \mathrm{y} \xi=5 \\
-\sqrt{\epsilon_{m_{y_{\gamma}}}}, \text { if } \gamma=3,4 \mathrm{y} \xi=5
\end{array}\right.
\end{aligned}
$$




$$
\begin{gathered}
\tau^{(\gamma, \xi)}=\left\{\begin{array}{l}
1, \text { if } \gamma=1,2 \mathrm{y} \xi=3,4 \\
-1, \text { if } \gamma=1,2,3,4 \mathrm{y} \xi=5
\end{array}\right. \\
\lambda_{m, n}^{(\gamma, \xi)}=\left\{\begin{array}{l}
k_{n_{x_{\xi}}}, \text { if } \gamma=1,2 \mathrm{y} \xi=3,4,5 \\
-k_{n_{y_{\xi}}}, \text { if } \gamma=3,4 \mathrm{y} \xi=5
\end{array}\right.
\end{gathered}
$$

In these expressions, $\epsilon_{n}$ is the Neumann factor defined in (A.6); $\delta_{m, n}$ is the Kronecker delta, and $\nu_{\xi}$ is defined in (4.156). 


\section{Appendix G}

\section{Publications related with the $\mathrm{PhD}$ thesis}

\section{Papers in international scientific journals:}

- J. Gil, A.M. Pérez, B. Gimeno, M. Bressan, V.E. Boria and G. Conciauro, “Analysis of cylindrical dielectric resonators in rectangular cavities using a state-space integralequation method," IEEE Microwave and Wireless Components Letters, vol. 16, pp.636638, December 2006.

- J. Gil, A.A. San Blas, C. Vicente, B. Gimeno, M. Bressan, V.E. Boria, G. Conciauro and M. Maestre, "Full-wave analysis and design of dielectric-loaded waveguide filters using a state-space integral-equation method," IEEE Transactions on Microwave Theory and Techniques, vol. 57, pp.109-120, January 2009.

- A.A. San Blas, F. Mira, J. Gil, V.E. Boria and B. Gimeno, "Efficient analysis and design of compensated turnstile junctions using advanced modal techniques," Progress In Electromagnetics Research Letters, vol. 12, pp.21-30, 2009.

\section{Publications in international congress:}

- J.Gil, A.A. San Blas, C. Vicente, B. Gimeno, M. Bressan, V.E. Boria, G. Conciauro and M. Maestre, "Analysis and design of waveguide filters with dielectric resonators using the state-space integral equation formulation," in Porc. XVII RiNEm Riunione Nazionale di Elettromagnetismo 2008, (Lecce, Italy), pp. 4, September 2008.

- S. Cogollos, V.E. Boria, J. Gil, C. Vicente and B. Gimeno, "FEST3D: A software tool for the analysis, synthesis and design of waveguide filters for satellite applications," in Proc. 2008 Applied Computational Electromagnetics Society (ACES) Conference, (Ontario, Canada), pp. 588-593, April 2008. 


\section{Bibliography}

[1] R. Levy, R. V. Snyder, and G. Matthaei, "Design of microwave filters," IEEE Trans. Microwave Theory Tech., vol. 50, pp. 783-793, March 2002.

[2] I. C. Hunter, L. Billonet, B. Jarry, and P. Guillon, "Microwave Filters-Applications and Technology," IEEE Trans. Microwave Theory Tech., vol. 50, pp. 794-805, March 2002.

[3] S. J. Fiedziuszko, I. C. Hunter, T. Itoh, Y. Kobayashi, T. Nishikawa, S. N. Stitzer, and K. Wakino, "Dielectric Materials, Devices, and Circuits," IEEE Trans. Microwave Theory Tech., vol. 50, pp. 706-720, March 2002.

[4] C. Wang and K. A. Zaki, "Dielectric Resonators and Filters," IEEE Microwave Magazine, pp. 115-127, October 2007.

[5] R. R. Mansour, "Filter Techonologies for Wireless Base Stations," IEEE Microwave Magazine, pp. 68-74, March 2004.

[6] R. R. Mansour, "High-Q Tunable Dielectric Resonator Filters," IEEE Microwave Magazine, pp. 84-98, October 2009.

[7] C. Kudsia, R. Cameron, and W.-C. Tang, "Innovations in microwave filters and multiplexing networks for communications satellite systems," IEEE Trans. Microwave Theory Tech., vol. 40, pp. 1133-1149, June 1992.

[8] R. D. Richtmyer, "Dielectric Resonators," Journal of Applied Physics, vol. 10, pp. 391-398, June 1939.

[9] A. Okaya and L. F. Barash, "The dielectric microwave resonator," Proc. IRE, vol. 50, pp. 2081-2092, October 1962.

[10] H. Y. Yee, "Natural resonant frequencies of microwave dielectric resonators," IEEE Trans. Microwave Theory and Techniques (Correspondence), vol. MTT-13, p. 256, March 1965.

[11] J. C. Sethares and J. Naumann, "Design of microwave dielectric resonators," IEEE Trans. Microwave Theory Tech., vol. MTT-14, pp. 2-7, January 1966. 
[12] W. H. Harrison, "A miniature high-Q bandpass filter employing dielectric resonators," IEEE Trans. Microwave Theory Tech., vol. 16, pp. 210-218, April 1968.

[13] S. B. Cohn, "Microwave filters containing high-Q dielectric resonators," in IEEE MTT-S Microwave Symp. Dig., May 1965.

[14] S. B. Cohn, "Microwave bandpass filters containing high-Q dielectric resonators," IEEE Trans. Microwave Theory Tech., vol. 16, pp. 218-227, April 1968.

[15] D. J. Masse, R. A. Pucel, D. W. Readey, E. A. Maguire, and C. P. Hartwig, "A new low loss high-k temperature compensated dielectric for microwave applications," in Proc. IEEE, vol. 59, pp. 1628-1629, November 1971.

[16] D. J. Masse and R. A. Pucel, "A temperature stable bandpass filter using dielectric resonators," in Proc. IEEE, vol. 60, pp. 730-731, June 1972.

[17] K. Wakino, T. Nishikawa, S. Tamura, and Y. Ishikawa, "Micorwave banpass filters containing dielectric resonators with improved temperature stability and spurious response," in IEEE MTT-S Int. Microwave Symp. Dig., vol. 30, pp. 63-65, 1975.

[18] J. K. Plourde and C.-L. Ren, "Applications of dielectric resonators in microwave components," IEEE Trans. Microwave Theory Tech., vol. 29, pp. 754-770, August 1981.

[19] D. Kajfez and P. Guillon, Dilectric Resonators. Artech House, Inc., 1986.

[20] S. J. Fiedziuszko, "Dual-mode dielectric resonator loaded cavity filters," IEEE Trans. Microwave Theory Tech., vol. 30, pp. 1311-1316, September 1982.

[21] Y. Kobayashi, N. Fukuoka, and S. Yoshida, "Resonant modes for a shielded dielectric rod resonator," Electronic and Communications in Japan, vol. 64-B, no. 11, pp. 46$51,1981$.

[22] K. A. Zaki and A. E. Atia, "Modes in dielectric-loaded waveguides and resonators," IEEE Trans. Microwave Theory Tech., vol. MTT-31, pp. 1039-1045, December 1983.

[23] K. A. Zaki and C. Chen, "New results in dielectric-loaded resonators," IEEE Trans. Microwave Theory Tech., vol. MTT-34, pp. 815-824, July 1986.

[24] T. Itoh and R. S. Rudokas, "New method for computing the resonant frequencies of dielectric resonators," IEEE Trans. Microwave Theory Tech., vol. 25, pp. 52-54, January 1977.

[25] Y. Konishi, N. Hoshino, and Y. Utsumi, "Resonant frequency of a $T E_{01 \delta}$ dielectric resonator," IEEE Trans. Microwave Theory Tech., vol. 24, pp. 112-114, February 1976.

[26] J. V. Bladel, "On the resonances of a dielectric resonator of very high permittivity," IEEE Trans. Microwave Theory Tech., vol. MTT-23, pp. 199-208, February 1975. 
[27] R. DeSmedt, "Correction due to a finite permittivity for a ring resonator in free space," IEEE Trans. Microwave Theory Tech., vol. MTT-32, pp. 1288-1293, October 1984.

[28] Y. Kobayashi and M. Miura, "Optimum design of shielded dielectric rod and ring resonators for obtaining the best mode separation," in IEEE MTT-S Int. Microwave Symp. Dig., (San Francisco), pp. 184-186, May-June 1984.

[29] S.-W. Chen and K. A. Zaki, "Dielectric ring resonators loaded in waveguide and on substrate," IEEE Trans. Microwave Theory Tech., vol. MTT-39, pp. 2069-2076, December 1991 .

[30] X.-P. Liang and K. A. Zaki, "Modeling of cylindrical dielectric resonators in recatangular waveguides and cavities," IEEE Trans. Microwave Theory Tech., vol. 41, pp. 2174-2181, December 1993.

[31] C. Wang, H.-W. Tao, K. A. Zaki, and R. R.Mansour, "Mixed modes cylindrical planar dielectric resonator filters with rectangular enclosure," IEEE Trans. Microwave Theory Tech., vol. 43, pp. 2817-2823, December 1995.

[32] C. Wang, K. A. Zaki, A. E. Atia, and T. G. Dolan, "Dielectric combline resonators and filters," IEEE Trans. Microwave Theory Tech., vol. 46, pp. 2501-2506, December 1998.

[33] J. A. Monsoriu, M. V. Andrés, E. Silvestre, A. Ferrando, and B. Gimeno, "Analysis of dielectric-loaded cavities using an orthonormal-basis method," IEEE Trans. Microwave Theory Tech., vol. 50, pp. 2545-2552, November 2002.

[34] J. A. Monsoriu, B. Gimeno, E. Silvestre, and M. V. Andrés, "Analysis of inhomogeneously dielectric filled cavities coupled to dielectric-loaded waveguides: application to the study of NRD-guide components," IEEE Trans. Microwave Theory Tech., vol. 52, pp. 1693-1701, July 2004.

[35] M. Polewsky, R. Lech, and J. Mazur, "Rigorous modal analysis of structures containing inhomogeneous dielectric cylinders," IEEE Trans. Microwave Theory Tech., vol. 52, pp. 1508-1516, May 2004.

[36] F. H. Gil and J. P. Martínez, "Analysis of dielectric resonators with tuning screw and supporting structure," IEEE Trans. Microwave Theory Tech., vol. MTT-33, pp. 14531457, December 1985.

[37] P. S. Kooi, M. S. Leong, and A. L. S. Prakash, "Finite-element analysis of the shielded cylindrical dielectric rsonator," in Proc. IEE, Pt. H, vol. 132, pp. 7-16, February 1985.

[38] D. Baillargeat, S. Verdeyme, M. Aubourg, and P. Guillon, "CAD applying the finiteelement method for dielectric-resonator filters," IEEE Trans. Microwave Theory Tech., vol. 46, pp. 10-17, January 1998. 
[39] J.-F. Lee, G. M. Wilkins, and R. Mittra, "Finite-element analysis of axisymmetric cavity resonator using a hybrid edge element tecnique," IEEE Trans. Microwave Theory Tech., vol. 41, pp. 1981-1987, November 1993.

[40] J. M. Gil, "CAD-oriented analisys of cylindrical and spherical dielectric resonators in cavities and MIC environments by means of finite elements," IEEE Trans. Microwave Theory Tech., vol. 53, pp. 2866-2874, September 2005.

[41] D. Rousset, P. Guillon, and Y. Garault, "Exact determination for the resonant frequencies and fields of dielectric resonators," in Porc. Europ. Microwave Conf., (Brighton), pp. 415-419, September 1979.

[42] P. Guillon, J. P. Balabaud, and Y. Garault, " $T M_{01 p}$ tubular and cylindrical dielectric resonator mode," in IEEE MTT-S Int. Microwave Symp. Dig., (Los Angeles), pp. 163166, June 1981.

[43] W. Yu and R. Mittra, "A conformal finite time domain technique for modeling curved dielectric surfaces," IEEE Microwave Wireless Compon. Lett., vol. 11, pp. 25-27, January 2001.

[44] N. Kaneda, B. Houshmand, and T. Itoh, "FDTD analysis of dielectric resonators with curved surfaces," IEEE Trans. Microwave Theory Tech., vol. 45, pp. 1645-1649, September 1997.

[45] M. Wiktor and M. Mrozowski, "Fast numerical analysis of dielectric resonators with perturbed rotational symmetry," IEEE Microwave Wireless Compon. Lett., vol. 15, pp. 516-518, August 2005.

[46] R. F. Harrington, Field Computation by Moment Methods. IEEE Press, Piscataway NJ, 1993.

[47] A. F. Peterson, S. L. Ray, and R. Mittra, Computational Methods for Electromagnetics. IEEE PRESS Series on Electromagnetic Waves, 1998.

[48] G. G. Raju, Dilectrics in Electric Fields. Marcel Dekker, Inc., 2003.

[49] R. F. Harrington, Time-harmonic Electromagnetic Fields. McGraw-Hill Book Company Inc., 1961.

[50] A. W. Glisson, D. Kajfez, and J. James, "Evaluation of modes in dielectric resonators using a surface integral equation formulation," IEEE Trans. Microwave Theory Tech., vol. MTT-31, pp. 1023-1029, December 1983.

[51] D. Kajfez, A. W. Glisson, and J. James, "Computed modal field distributions for isolated dielectric resonators," IEEE Trans. Microwave Theory Tech., vol. MTT-32, pp. 1609-1616, December 1984. 
[52] A. S. Omar and K. Schünemann, "Scattering by material and conducting bodies inside waveguides, part I: Theoretical formulations," IEEE Trans. Microwave Theory Tech., vol. MTT-34, pp. 266-272, February 1986.

[53] S.-L. Lin and G. W. Hanson, "An efficient full-wave method for analysis of dielectric resonators possessing separable geometries immersed in inhomogeneous environments," IEEE Trans. Microwave Theory Tech., vol. 48, pp. 84-92, January 2000.

[54] A. A. Kishk, D. Kajfez, and S. Chebolu, "Resonant frequency and Q factor of axisimmetric composite microwave cavities," IEEE Trans. Microwave Theory Tech., vol. 50, pp. 2287-2293, October 2002.

[55] V. Catina, F. Arndt, and J. Brandt, "A surface integral equation formulation for dielectric post structures in waveguides," in IEEE MTT-S Int. Microwave Symp., (Long Beach, CA), June 2005.

[56] V. Catina, F. Arndt, and J. Brandt, "Hybrid surface integral-equation/mode-matching method for the analysis of dielectric loaded waveguide filters of arbitrary shape," IEEE Trans. Microwave Theory Tech., vol. 53, pp. 3562-3567, November 2005.

[57] S. M. Rao, D. R. Wilton, and A. W. Glisson, "Electromagnetic Scattering by surfaces of arbitrary shape," IEEE Trans. Antennas Propagat., vol. AP-30, pp. 409-418, May 1982.

[58] F. Alessandri, M. Chiodetti, A. Giugliarelli, D. Maiarelli, G. Martirano, D. Schmitt, L. Vanni, and F. Vitulli, "The electric-field integral-equation method for the analysis and design of a class of rectangular cavity filters loaded by dielectric and metallic cylindrical pucks," IEEE Trans. Microwave Theory Tech., vol. 52, pp. 1790-1797, August 2004.

[59] T. Itoh, Numerical techniques for microwaves and millimeter-wave passive structures. John Wiley \& Sons, New York, 1989.

[60] J. E. Bracken, D.-K. Sun, and Z. J. Cendes, "S-Domain Methods for Simultaneous Time and Frequency Characterization of Electromagnetic Devices," IEEE Trans. Microwave Theory Tech., vol. 46, pp. 1277-1290, September 1998.

[61] F. P. and F. R. W., "Efficient linear circuit analysis by Padè approximation via the Lanczos process," IEEE Trans. Computer-Aided Design, vol. 14, pp. 639-649, September 1995.

[62] G. Conciauro, M. Guglielmi, and R. Sorrentino, Advanced Modal Analysis. John Wiley \& Sons, 2000.

[63] P. Arcioni, M. Bressan, and L. Perregrini, "A new boundary integral approach to the determination of the resonant modes of arbitrary shaped cavities," IEEE Trans. Microwave Theory Tech., vol. 43, pp. 1848-1856, August 1995. 
[64] G. Conciauro, M. Bressan, and C. Zuffada, "Waveguide Modes Via an Integral Equation Leading to a Linear Matrix Eigenvalue Problem," IEEE Trans. Microwave Theory Tech., vol. MTT-32, pp. 1495-1504, November 1984.

[65] S. Cogollos, S. Marini, V. E. Boria, P. Soto, A. Vidal, H. Esteban, J. V. Morro, and B. Gimeno, "Efficient modal analysis of arbitrarily shaped waveguides composed of linear, circular, and elliptical arcs using the BI-RME method," IEEE Trans. Microwave Theory Tech., vol. 51, pp. 2378-2390, December 2003.

[66] G. Conciauro, P. Arcioni, M. Bressan, and L. Perregrini, "Wideband Modelling of Arbitrarily Shaped H-Plane Waveguide Components by the Boundary Integral-Resonant Mode Expansion Method ," IEEE Trans. Microwave Theory Tech., vol. 44, pp. 10571066, July 1996.

[67] G. Conciauro, P. Arcioni, M. Bressan, and L. Perregrini, "Wideband Modelling of Arbitrarily Shaped E-Plane Waveguide Components by the Boundary Integral-Resonant Mode Expansion Method ," IEEE Trans. Microwave Theory Tech., vol. 44, pp. 20832092, November 1996.

[68] P. Arcioni, M. Bozzi, M. Bressan, and L. Perregrini, "A novel CAD tool for the wideband modeling of 3D waveguide components," International Journal of RF and Microwave Computer Aided Engineering, vol. 10(3), pp. 183-189, 2000.

[69] P. Arcioni, M. Bressan, G. Conciauro, and L. Perregrini, "Generalized Y-Matrix of Arbitrary H-plane Waveguide Junctions by the BI-RME Method," in 1997 IEEE MTTS Int. Microw. Symp. Dig., (Denver, CO), pp. 1269-1272, June 1997.

[70] P. Arcioni, M. Bressan, and G. Conciauro, "Generalized Y-Matrix of Arbitrary Eplane Waveguide Junctions by the BI-RME Method," in 1999 IEEE MTT-S Int. Microw. Symp. Dig., (Anaheim, CA), pp. 1269-1272, June 1999.

[71] M. Bozzi, M. Bressan, and L. Perregrini, "Generalized Y-Matrix of Arbitrary 3D Waveguide Junctions by the BI-RME Method," in 1999 IEEE MTT-S Int. Microw. Symp. Dig., (Anaheim, CA), pp. 1269-1272, June 1999.

[72] P. Arcioni and G. Conciauro, "Combination of Generalized Admittance Matricesin the Form of Pole Expansions," IEEE Trans. Microwave Theory Tech., vol. 47, pp. 19901996, October 1999.

[73] P. Arcioni, M. Bozzi, M. Bressan, G. Conciauro, and L. Perregrini, "Frequency/timedomain modeling of 3-D waveguide structures by a BI-RME approach," Int. J. Numer. Modeling, vol. 15, pp. 3-21, January 2002.

[74] F. Mira, M. Bressan, G. Conciauro, B. Gimeno, and V. E. Boria, "Fast S-domain modeling of rectangular waveguides with radially symmetric metal insets," IEEE Trans. Microwave Theory Tech., vol. 53, pp. 1294-1303, April 2005. 
[75] A. A. San Blas, F. Mira, V. E. Boria, B. Gimeno, M. Bressan, and P. Arcioni, "On the fast and rigorous analysis of compensated waveguide junctions using off-centered partial-height metallic posts," IEEE Trans. Microwave Theory Tech., vol. 55, pp. 168175, January 2007.

[76] V. E. Boria, M. Bozzi, D. Camilleri, A. Coves, H. Esteban, B. Gimeno, M. Guglielmi, and L. Polini, "Contributions to the analysis and design of all-inductive filters with dielectric resonators," in Proc. of EUMW 2003 European Microwave Week, (Munich), pp. 1247-1250, Oct. 2003.

[77] M. Bressan, G. Conciauro, and W. Eyssa, "Integral equation method for S-domain modelling of rectangular waveguides with dielectric insets (2D case)," in 2006 IEEE MTT-S Int. Microw. Symp. Dig., pp. 1049-1052, June 2006.

[78] M. Bressan, G. Conciauro, and W. Eyssa, "S-domain modelling of rectangular waveguides with dielectric insets (3D case)," in 2006 IEEE Int. S. on Antenna Propagation \& EM Theory, ISAPE'2006, pp. 1-4, October 2006.

[79] M. Bressan, G. Conciauro, and W. Eyssa, "Scattering from Multi-Dielectric Insets in Rectangular Waveguides by a Novel State-Space Integral-Equation Method," in 2007 IEEE Int. S. on Antennas and Propagation, pp. 2789-2792, June 2007.

[80] Ansoft High Frequency Structure Simulator (Ansoft HFSS). http://www.ansoft.com.

[81] FEST3D (Full-wave Electromagnetic Simulation Tool 3D). http://www.fest3d.com.

[82] M. Bressan and G. Conciauro, "Rapidly Converging Expressions of Electric Dyadic Green's Functions for Resonators," in Proc. 1983 URSI Symp on Electromagnetic Theory (Santiago de Compostela, Spain), pp. 41-44, August 1983.

[83] K. Kurokawa, "The Expansions of Electromagnetic Fields in Cavities," IRE Trans. Microwave Theory Tech., vol. MTT-6, pp. 178-187, April 1958.

[84] K. Kurokawa, An Introduction to the Theory of Microwave Circuits. Academic Press, New York, 1969.

[85] J. V. Bladel, Electromagnetic Fields. Hemisphere, Washington, 1985.

[86] R. E. Collin, Field Tehory of Guided Waves. IEEE Press, New York, 1990.

[87] Y. Rahmat-Samii, “On the Question of Computation of the Dyadic Green's Function at the Source Region in Waveguides and Cavities," IEEE Trans. Microwave Theory Tech., vol. MTT-23, pp. 762-765, September 1975.

[88] C.-T. Tai and P. Rozenfeld, "Different Representations of Dyadic Green's Functions for a Rectangular Cavity," IEEE Trans. Microwave Theory Tech., vol. MTT-24, pp. 597-601, September 1976. 
[89] C.-T. Tai, Dyadic Green Functions in Electromagnetic Theory. IEEE Press, New York, 1993.

[90] J. V. Bladel, “Some Remarks on Green's Dyadic for Infinite Space," IRE Rrans. Antennas Propagat., vol. AP-9, pp. 563-566, November 1961.

[91] A. D. Yaghjian, "Electric Dyadic Green's Functions in the Source Region," Proceedings of the IEEE, vol. 68, pp. 248-263, February 1980.

[92] S.-W. Lee, J. Boersma, C.-L. Law, and G. A. Deschamps, "Singularity in Green's Function and its Numerical Evaluation," IEEE Trans. Antennas Propagat., vol. AP28, pp. 311-317, May 1980.

[93] M. Kisliuk, "The dyadic Green's fynctions for cylindrical waveguides and cavities," IEEE Trans. Microwave Theory Tech., vol. MTT-28, pp. 894-898, August 1980.

[94] A. Q. Howard and B. D. Seidel, "Singularity extraction in kernel fucntions in closed region problems," Radio Science, vol. 13, pp. 425-429, May-June 1978.

[95] V. G. Daniele and M. Orefice, "Dyadic Green's Functions in Bounded Media," IEEE Trans. Antennas Propagat., vol. AP-32, pp. 193-196, February 1984.

[96] M. Bressan and G. Conciauro, "Rapidly Converging Expressions for Dyadic Green's Functions in two-dimensional Resonators of Circular and Rectangular cross-section," Alta Frequenza, vol. LII, pp. 188-190, May-June 1983.

[97] M. Bressan and G. Conciauro, "Singularity Extraction from the Electric Green's Function for a Spherical Resonator," IEEE Trans. Microwave Theory Tech., vol. MTT-33, pp. 407-414, May 1985.

[98] O. D. Kellogg, Foundations of Potential Theory. Springer Verlag, New York, 1967.

[99] C. A. Balanis, Advanced Engineering Electromagnetics. John Wiley \& Sons, 1989.

[100] P. M. Morse and H. Feshbach, Methods of Theoretical Physics. McGraw Hill Book Company Inc., 1953.

[101] M. Bressan, L. Perregrini, and E. Regini, "BI-RME modeling of 3D waveguide components enhanced by the Ewald technique," in 2000 IEEE MTT-S Int. Microw. Symp. Dig., pp. 1097-1100, June 2000.

[102] P. Ewald, "Die Berechnung Optischer und Electroststischer Gitterpotentiale," Ann. der Physik, vol. 64, 1921.

[103] F. Mira, Análisis modal eficiente de guías de onda rectangulares con postes conductores. Tesis Doctoral, Universidad Politécnica de Valencia, 2005. 
[104] E. Regini, Analisi si Cavità Risonanti con il Metodo BIRME in Geometria Trirettangolare. Tesi di Laurea, Università di Pavia, 1998-99.

[105] J. N. Franklin, Matrix Theory. Prentice-Hall Inc., Englewood Cliffs, NJ, 1968.

[106] P. Arcioni, M. Bressan, and L. Perregrini, "On the evaluation of the double surface integrals arising in the application of the boundary integral method to 3-D problems," IEEE Trans. Microwave Theory Tech., vol. 45, pp. 436-439, August 1995.

[107] N. Marcuvitz, Waveguide Handbook. Peter Peregrinus Ltd., London, 1986.

[108] J. Krupka, "Resonant modes in Shielded Cylindrical Ferrite and Single-Crystal Dielectric Resonators," IEEE Trans. Microwave Theory Tech., vol. 37, pp. 691-697, April 1989.

[109] E. Snitzer, "Cylindrical dielectric waveguide modes," Journal of the Optical Society of America, vol. 51, pp. 491-498, May 1961.

[110] K. S. Packard, "The origin of waveguides: A case of multiple rediscovery," IEEE Trans. Microwave Theory Tech., vol. 32, pp. 961-969, September 1984.

[111] J. P. Astier and P. Guillon, "Elliptic microwave filter using dual modes of dielectric resonators," in European Microwave Conf. Dig., pp. 335-340, Sept. 1985.

[112] K. A. Zaki, C. Chen, and A. E. Atia, "Canonical and longitudinal dual-mode dielectric resonator filters," IEEE Trans. Microwave Theory Tech., vol. MTT-35, pp. 1130-1134, December 1987.

[113] Y. Kobayashi and K. Kubo, "Canonical bandpass filters using dual-mode dielectric resonators," in IEEE MTT-S Int. Microwave Symp. Dig., pp. 137-140, 1987.

[114] I. C. Hunter, D. J. Rhodes, and V. Dassonville, "Dual-mode filters with conductor loaded dielectric resonators," IEEE Trans. Microwave Theory Tech., vol. 42, pp. 2304 2311, December 1999.

[115] V. Walker and I. C. Hunter, "Design of triple mode $T E_{01}$ resonator transmission filters," IEEE Microwave Wireless Compon. Lett., vol. 12, pp. 215-217, June 2002.

[116] J. Hattori, T. Wada, H. Kubo, and Y. Ishikawa, "2 GHz band Quadruple mode dielectric resonator filter for cellular base stations," in IEEE MTT-S Int. Microwave Symp Dig., (Philadelphia, PA), pp. 933-936, 2003.

[117] M. Mattes, Contribution to the Electromagnetic Modelling and Simulation of Waveguide Networks Using Integral Equations and Adaptive Sampling. PhD Thesis. École Polytechnique Fédérale de Lausanne, CH-1015 Lausanne, Switzerland. Thesis No. 2693, 2003. 
[118] V. E. Boria, S. Cogollos, H. Esteban, M. Guglielmi, and B. Gimeno, "Efficient analysis of a cubic junction of rectangular waveguides using the admittance-matrix representation," in IEE Proceedings - Microwave Antennas and Propagation, vol. 147, pp. 417-422, 2000.

[119] C. L. Ren, "Waveguide bandstop filter utilizing $\mathrm{Ba}_{2} \mathrm{Ti}_{9} \mathrm{O}_{20}$ resonators," in IEEE MTTS Int. Microwave Symp. Dig., pp. 227-229, 1978.

[120] A. A. S. Blas, Análisis y diseño de dispositivos pasivos de microondas y milimétricas en guía de ondas con excitación coaxial. Tesis Doctoral, Universidad Politécnica de Valencia, 2008. 